\title{
Seismic and Magnetic Constraints on the Structure of Upper Oceanic Crust at Fast and Slow Spreading Ridges
}

\author{
by \\ Stefan Anthony Hussenoeder \\ B.S., Geophysics \\ Saint Louis University, 1992
}

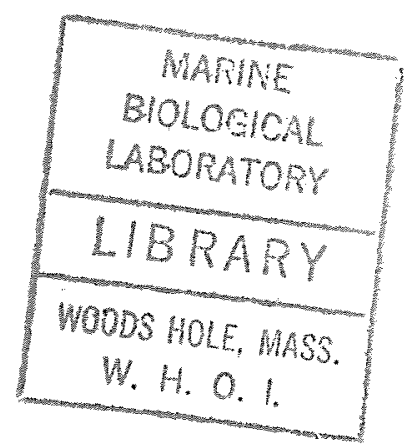

\begin{abstract}
Submitted in partial fulfillment of the requirements for the degree of
Doctor of Philosphy

at the

MASSACHUSETTS INSTITUTE OF TECHNOLOGY

and the

WOODS HOLE OCEANOGRAPHIC INSTITUTION
\end{abstract}

September 1998

(C)1998 Stefan A. Hussenoeder. All rights reserved

The author hereby grants to MIT and to WHOI permission to reproduce and distribute publicly paper and electronic copies of this thesis document in whole or in part, and to grant others the right to do so.

Signature of Author

Joint Program in Oceanography, Massachusetts Institute of Technology and

Woods Hole Oceanographic Institution, August 7, 1998

Certified by-

Graham M. Kent, Robert S. Detrick, Maurice A. Tivey Assistant Research Geophysicist, Senior Scientist, Associate Scientist Thesis Co-Advisors

Accepted by_

Deborah K. Smith, Chair, Joint Committee for Marine Geology and Geophysics, Massachusetts Institute of Technology and Woods Hole Oceanographic Institution 


\title{
Seismic and Magnetic Constraints on the Structure of Upper Oceanic Crust at Fast and Slow Spreading Ridges
}

\author{
by \\ Stefan Anthony Hussenoeder \\ Submitted to the MIT/WHOI Joint Program in Oceanography \\ on August 7, 1998, in partial fulfillment of the \\ requirements for the degree of Doctor of Philosophy
}

\begin{abstract}
The upper ocean crust contains a comprehensive record of the shallow geological processes active along the world's mid-ocean ridge system. This thesis examines the magnetic and seismic structure of the upper crust at two contrasting ridges-the fast spreading East Pacific Rise (EPR) and the slow spreading Mid-Atlantic Ridge (MAR) - to build a more complete understanding about the roles of volcanic emplacement, tectonic disruption and hydrothermal alteration in the near-ridge environment.

A technique that inverts potential field measurements directly from an uneven observation track is developed and applied to near-bottom magnetic data from the spreading segments north of the Kane transform on the MAR. It is concluded that the central anomaly magnetization high marks the locus of focused volcanic emplacement. A cyclic faulting model is proposed to explain the oscillatory magnetization pattern associated with discrete blocks of crust being transported out of the rift valley between intensely altered fault zones. Seismic waveform and amplitude analyses of the magma sill along the EPR reveal it to be a thin $(<100 \mathrm{~m})$ body of partial melt. These characteristics have important implications for melt availability and transport within the cycle of eruption and replenishment. A genetic algorithm-based seismic waveform inversion technique is developed and applied to on- and near-axis multichannel data from $17^{\circ} 20^{\prime} \mathrm{S}$ on the EPR and the spreading segment south of the Oceanographer transform (MAR) to map and compare for the first time the detailed velocity structure of the upper crust at two different spreading rates. Combined with conventionally processed seismic profiles, our results show that, while final extrusive thickness is comparable at all spreading ridges $(300-500 \mathrm{~m})$, the style of thickening may vary. While a thin $(\leq 100 \mathrm{~m})$ extrusive carapace quadruples in thickness within 1-4 km of the EPR crest, the extrusive section at the MAR achieves its final thickness within the inner valley. Both show evidence for a narrow zone of volcanic emplacement. Vigorous hydrothermalism at the EPR may produce a more rapid increase in basement velocities relative to the MAR. Rapid modification of the extrusive/dike transition at both ridges indicates that hydrothermalism is enhanced in this interval. Along-axis transport of lavas may thicken the extrusive pile at slow spreading segment ends, strengthening the magnetic highs generated by lava chemistry.
\end{abstract}

Thesis Supervisors: Graham M. Kent, Robert S. Detrick, Maurice A. Tivey Titles: Assistant Research Geophysicist, Senior Scientist, Associate Scientist 


\section{Biographical Sketch}

In order to wax only partially rhapsodic, let me start at my formative age as a marine geophysicist...the age of six. The first hint that I wasn't going to grow up to be a successful businessman was dropped in Germany, where my best friend and I rejected the idea of opening a lemonade stand in favor of selling fossils and geodes on the street corner. We soon went bankrupt. Our village in southern Germany was not far from Solnhofen, home of the famed Archaeopteryx. The countryside was full of geological treasures exposed in streambeds or along roadsides. Our final move in 1978 from frequent trips along the Meditterranean to my mother's home in land-locked, tectonically handicapped (yet beautiful in its own way) Nebraska probably motivated my interest in the suddenly distant sea. Music and science dominated my activities in high school, near the end of which I realized that I stood at a proverbial fork in the road: pursue the life of a musician or attempt a career as a scientist. I chose science, and having retained my affinity for rocks and the sea, I chose marine geophysics. Professors at prospective colleges advised me that the best way to prepare for this career was to become well-versed in its fundamentals: mathematics, physics and geology. So, I soon began a curriculum in geophysics (with a music performance minor) at Saint Louis University. My strangely farsighted intent was to later take a bachelor's degree to WHOI and earn a Ph.D. in the marine geophysics (hmm...). I remember sitting at the living room piano my senior year in high school, announcing these future plans and seeing my parents grapple with the (misinformed) prospect of paying for ten more years of school. Four years later, after visiting and nearly accepting offers from Scripps and the University of Washington, I attended the open house at MIT/WHOI and soon found myself following the path I had originally planned. I moved to Woods Hole. In an effort to counteract my weak tendency to be a computer hermit in the Joint Program, I have attempted to retain some extracurricular activities by performing in singing and instrumental groups and playing sports such as underwater hockey (yes, it's a sport). The majority of time spent away from my office, however, has still revolved around my research interests in the form of field expeditions, research cruises and conferences. Now I have my Ph.D. from WHOI, and I'm off to join Exxon Production Research in Houston. So, what's the plan? 


\section{Curriculum Vita \\ Stefan Anthony Hussenoeder \\ Joint Program in Oceanography \\ Woods Hole Oceanographic Institution}

Birth: September 1, 1970. Madrid, Spain; US citizen

\section{Research Interests:}

The origin and maturation of oceanic crust; magma distribution and transport, dike emplacement, eruptive processes, tectonic evolution and hydrothermal alteration. The development and application of seismic and magnetic techniques in the study of geophysical processes. Optimization methods in geophysical inversion.

\section{Education:}

1992-present Ph.D. candidate, Marine Geophysics, MIT/WHOI Joint Program in Oceanography

1992 B.S. summa cum laude, Geophysics with Music Performance minor, Saint Louis University, St. Louis, Missouri

\section{Academic Honors:}

1996

1992-1995

1991

1988

1988

1988
Outstanding Student Paper, Fall AGU-Tectonophysics Section Office of Naval Research Fellowship Alpha Sigma Nu Jesuit Honor Society Society of Exploration Geophysicists Academic Merit Scholarship St. Louis University Macelwaine/Blum Geophysics Scholarship St. Louis University Dean's Scholarship

\section{Languages:}

Fluent in English and German

Computer Languages: FORTRAN, C, MATLAB, Perl

Computer Operating Systems: UNIX, DOS, Macintosh

\section{Employment and Experience:}

1996 Graduate Teaching Assistant, WHOI

1992-present Graduate Research Assistant, WHOI

1991 Summer Research Fellow, Scripps Institute of Oceanography

1990-1992 Physics Teaching Assistant, Saint Louis University

\section{Professional Activities:}

1994-1996 Student Representative, WHOI

1993-present Member, American Geophysical Union

1988-1993 Member, Society of Exploration Geophysicists 


\section{Research Accomplishments:}

\section{Publications:}

Tivey, M. A., H. P. Johnson, and BLANCOVIN Scientific Party (C. Fleutelot, S. Hussenoeder, R. Lawrence, D. D. Naidoo, D. Van Patten, C. Waters and B. Wooding), Direct measurements of magnetic reversal polarity boundaries in a crosssection of oceanic crust, submitted to Geophys. Res. Lett., 1998

Kent, G., A. Harding, S. Singh, M. Sinha, P. Barton, J. Orcutt, R. White, and ARAD Group Participants (H. Avendonk, S. Bazin, D. Boschi, A. Cherrett, A. Greer, P. Henkart, R. Hobbs, C. Hollinshead, S. Hussenoeder, T. Owen, V. Tong and P. Zimmer), The $A R A D$ 3-D seismic experiment: A detailed reflection and tomographic investigation of the $9^{\circ} 03^{\prime} \mathrm{N}$ overlapping spreading center, East Pacific Rise, InterRidge News, 7(1), 33-39, 1998.

Detrick, R., J. Collins, G. Kent, J. Lin, D. Toomey, A. Barclay, E. Hooft, A. Hosford and S. Hussenoeder, Mid-Atlantic Ridge Bullseye Experiment: A seismic investigation of segment-scale heterogeneity at a slow-spreading ridge, InterRidge News, 6(1), 27-32, 1997

Hussenoeder, S. A., M. A. Tivey and H. Schouten, Near-bottom magnetic survey of the Mid-Atlantic Ridge axis, $24^{\circ}-24^{\circ} 40^{\prime} \mathrm{N}$ : Implications for crustal accretion at slow spreading ridges, J. Geophys. Res., 101, 22051-22069, 1996

Hussenoeder, S. A., J. A. Collins, G. M. Kent, R. S. Detrick and the TERA Group, Seismic analysis of the axial magma chamber reflector along the East Pacific Rise from conventional reflection profiling, J. Geophys. Res., 101, 22087-22105, 1996

Kleinrock, M. C., S. E. Humphris and the Deep-TAG Team (P. Shaw, A. Bowen, T. Crooke, C. Davis, R. Elder, D. Gleason, J. Goff, L. Goldstein, W. Handley, J. Howland, S. Hussenoeder, K. Koga, S. Lerner, K. Nakamura, M. Rashid, L. Reiser Wetzel, W. Sellers, M. Sulanowska, C. Van Dover and L. Whitcomb), 2. Detailed structure and morphology of the TAG active hydrothermal mound and its geotectonic environment, in S. E. Humphris et. al. (eds.), Proceed. Ocean Drill. Prog. Init. Rep., 158, 15-21, 1996

Hussenoeder, S. A., M. A. Tivey and H. Schouten, Direct inversion of potential fields from an uneven track with application to the Mid-Atlantic Ridge, Geophys. Res. Lett., 22, 3131-3134, 1995

\section{Abstracts:}

Hussenoeder, S. A., R. S. Detrick, G. M. Kent and A. J. Harding, Mid-Atlantic Ridge Bullseye Experiment: Fine-scale structure of extrusive crust from multichannel seismics, Eos, Trans. AGU, 78(46), 641, 1997

Hussenoeder, S. A., and G. M. Kent, Seismic constraints on the shallow crustal architecture of mid-ocean ridges (Invited), Eos, Trans. AGU, 78(46), 692, 1997

Hussenoeder, S. A., G. M. Kent, R. S. Detrick and A. J. Harding, The structure of the volcanic crust at fast and slow spreading mid-ocean ridges, Ridge Summer School on Active Processes at Mid-Ocean Ridges, Lake Myvatn, Iceland, 1997 
Hussenoeder, S. A., G. M. Kent, R. S. Detrick and A. J. Harding, A comparison of upper crustal structure between fast and slow spreading ridges from genetic algorithm seismic waveform inversion, Eos, Trans. AGU, 77(46), 729, 1996

Kent, G. M., S. A. Hussenoeder, J. A. Collins, and R. S. Detrick, Is the melt lens a mush lens?, Ridge Theoretical Institute, Lake Tahoe, NV, 1995

Kleinrock, M. C., S. E. Humphris, and the Deep-TAG Team (P. Shaw, A. Bowen, T. Crooke, C. Davis, R. Elder, D. Gleason, J. Goff, L. Goldstein, W. Handley, J. Howland, S. Hussenoeder, K. Koga, S. Lerner, K. Nakamura, M. Rashid, L. Reiser Wetzel, W. Sellers, M. Sulanowska, C. Van Dover and L. Whitcomb), Detailed morphology and distribution of venting at the active TAG hydrothermal mound, $26^{\circ} \mathrm{N}$, Mid-Atlantic Ridge, Eos, Trans. AGU, 76(46), 1995

Hussenoeder, S. A., J. A. Collins, G. M. Kent and R. S. Detrick, Evidence for a thin, partially molten sill beneath the East Pacific Rise from seismic modeling of the AMC reflection, Eos, Trans. AGU, 76(46), 595, 1995

Tivey, M. A., C. Fleutelot, S. Hussenoeder, H. P. Johnson, R. M. Lawrence, D. D. Naidoo, D. Van Patten, C. Waters and F. B. Wooding, BLANCOVIN: A submersible study of the oceanic crust at a magnetic polarity reversal boundary, Eos, Trans. AGU, 76(46), 421,1995

Hussenoeder, S. A., M. A. Tivey and H. Schouten, Near-bottom magnetic survey of the Mid-Atlantic Ridge axis, $24^{\circ}-24^{\circ} 40^{\prime} \mathrm{N}$ (Invited), IUGG XXI General Assembly, Boulder, CO, 1995

Hussenoeder, S. A., M. A. Tivey and H. Schouten, Near-bottom magnetic survey of the Mid-Atlantic Ridge axis, $24^{\circ}-24^{\circ} 40^{\prime} \mathrm{N}$, Eos, Trans. AGU, 74(44), 574, 1993

\section{Field Work:}

$1997 R / V$ Maurice Ewing -3D multichannel and ocean-bottom seismic survey at $9^{\circ} \mathrm{N}$ (EPR), G. Kent, P.I.

1997 Field Work-Iceland, with H. Dick, B. Tucholke

1997 Field Work-Oman Ophiolite, with P. Kelemen, G. Hirth

$1996 R / V$ Maurice Ewing-Gravity, magnetic, OBS, and MCS study south of the Oceanographer transform (MAR), R. Detrick, P.I.

1996 Field Work-Hawaiian Islands, with H. Dick, H. Schouten, K. Johnson

$1995 R / V$ Atlantis $I I-$ Near-bottom and sea surface gravity-magnetic study of the Blanco Scarp (JDFR), ALVIN and ABE vehicles, M. Tivey, P.I.

1994 Field Work-Basin and Range, USA, with G. Hirth, B. Tucholke

$1994 R / V$ Knorr-Near-bottom study of the TAG hydrothermal segment (MAR), DSL120 and ARGO-II, M. Kleinrock, S. Humphris co-P.I.s

1993 Field Work-Bay of Islands Ophiolite, Newfoundland, with H. Dick, H. Schouten, B. Tucholke

$1993 R / V$ Knorr - Near-bottom study of ARSRP area west of the MAR, DSL120 and JASON ROV, B. Tucholke, P.I. 


\section{Acknowledgments}

Support for my graduate research has come from several funding sources, for which I am grateful. As an ONR graduate fellow during my first three years, I enjoyed having my own funds and the freedom of choice that came with it. The magnetics portion of this thesis was also supported by NSF grants OCE-9204141, OCE-9200905 (M. A. Tivey \& H. Schouten) and NERC GR3/7702 (R. C. Searle). Research for Chapter 4 was partially funded by NSF grant OCE-9402933 (R. S. Detrick). The final two science chapters were supported by NSF grants OCE-9012707, OCE-9300450 (R. S. Detrick), OCE-9401717 (G. M. Kent \& R. S. Detrick), OCE-9400623 (M. A. Tivey) and the Education office. I would especially like to thank the G\&G and Education offices for their non-monetary support and constant willingness to fix any administrative problems (especially when it was me that screwed up).

This thesis made ample use of the SIOSEIS processing package, developed by Paul Henkart, and PLTSEGY, developed by Alistair Harding. Jim Dolan and Dave Dubois kept the hardware running in the OBS lab and at sea. Without the help of these people, the quality of this thesis would certainly have suffered.

To my co-advisors (Robert Detrick, Graham Kent, Maurice Tivey), I owe many thanks. I have enjoyed working with them immensely and have always felt fortunate to be advised at a level appropriate to the academic/intellectual stage I was operating at. Now, with six years of hindsight, I believe I could not have chosen a better group of mentors to guide me through this rewarding graduate career. Similarly, John Collins and Hans Schouten have provided excellent guidance at various stages of my research, and are, as far as I am concerned, also thesis co-advisors.

For the many adventurous and informative field trips, I thank Henry Dick and the Geodynamics Seminar, Parker Hackett, Gregg Hirth and Peter Kelemen. The Oman trip in particular was an experience I will never forget (anala atakalam al arabi!).

Thanks go to the Joint Program and the crew/scientists aboard the R/Vs Atlantis II, Knorr, Maurice Ewing, and the Corwith Cramer for allowing me to experience the wonders of being at sea. From diving in the submersible Alvin to OBH recoveries from the Zodiac (and port calls at either end), I will treasure those memories always.

My high school years were blessed with an unforgettable group of characters, whose friendship has not diminished with time. Special thanks go to Aaron Comer, Robin (Adams) Cooper, Helen Konstantopoulos, Karey (Lindholm) Knaus, Jerry \& Bobbie (Adams) Kuker, Jamie Loss, Mitzi (Deckinger) Luedtke, Mary (Elwonger) Lunsford, Loyal \& Katie Parsons, Ueli Schaub, David \& Brenda (Lampe) Schwartz, Aaron \& June (Strasil) Tilton and and Steve Walker. Thank you all for the Playschool doctor's bag (and its...contents).

I would like to thank my college roommates of $2+$ years (Ed Baugh, David Behrns, David Eller, Doug Sanders, Tim Trentler), the residents of 4-Reinert (particularly Matt Hagemann, Cheryl (Buse) Lechnowsky, Gregg Nelson, Karen Spitzig and Michelle Whitfield) and the Mastersingers for their friendship and a healthy mix of 
wholesome activities and reckless irresponsibility. Guy Smith, my undergraduate advisor, first sparked my interest in marine magnetics. The instruction of Fred Chester and Brian Mitchell were instrumental in my preparation for graduate school. I would also like to thank Bernard Minster and Jason Phipps Morgan at Scripps for letting me play in a sand-box the entire summer of ' 91 and still call it a research project.

Many friends at WHOI have helped make this six-year trek to a Ph.D. not only bearable but enjoyable-from the houses of Angst (Liz Minor, Kathy Barbeau, Laura Magde, Sue Bello) and Good Food (Jay Austin, Jamie Pringle, Kelsey Jordahl), Tall/Dark/Handsome (Kirsten Laarkamp/Lihini Aluwihare/Dana Stuart), Millfield, and the Attic (Sarah Zimmerman et al.) to Diane DiMassa (doctor without a house), Craig Lewis, Miles Sundermeyer, Rebecca Thomas and many others. At work, I have greatly benefitted from interactions with students and faculty alike: Mark Behn, Mike Braun, Pablo Canales, Javier Escartin, Dan Fornari, Robert Greaves, Debbie Hassler, Emilie Hooft, Susan Humphris, Gary Jaroslow, Jennifer Jeorgen, Marty Kleinrock, Jun Korenaga, Jian Lin, Dan Lizaralde, Marcia McNutt, Debbie Smith, Brian Tucholke and Rob Van der Hilst to name several.

Special thanks go to my former housemates at Tang Hall, 85 Oyster Pond, 1 Cedar Street (the rat shack), 490 Woods Hole Road (the boarding house), and especially Bill Williams and Tad Snow (in our "excellent adventure" at 58 Oyster Pond).

I have had the pleasure of sharing offices with two fellow students and all around great human beings: Garrett Ito and Allegra Hosford. Thanks for being there to share ideas, critiques, thesis trials/tribulations and general office banter. I wish you all the best in your pursuit of scientific and personal nirvana.

Without music and (under)water sports, life in Woods Hole would have been much less rosy. They provided hours of much needed diversion and have enriched my life considerably. A "note" of thanks goes out to those songmeisters (Solstice Singers, Cappella Cantorum, Notescape.cod, and the Testostertones-Mark Hill, Mario Sengco, Bruce Woodin) and aquaphiles, whose companionship I have enjoyed as a result of our common interests.

To acknowledge some vices that helped me through, I must at least mention CocaCola $_{\circledast}$, chocolate-chip cookies and curries (the three C's) - two of which I wish were never invented, but am greatly indebted to nevertheless.

No one has meant more to me or given me more happiness than Kirsten Laarkamp. Her love and kindness, monumental patience at times, and, yes, spunk! have been an inspiration to me and are qualities that I increasingly admire and love with each passing day.

I will always be grateful for my parents, Angela and Günter, and my sisters, Regina and Sabrina, who I am certain constitute, along with Agnes Zorn (Oma) and Sister Catherine Coupe (Tiny), the best family in the world. Their unconditional love and support have been the foundation of my life, and I love them dearly.

This thesis was written in the memory of my grandparents, Ernst and Anne $\mathrm{Hu}$ ßenöder and Floyd Zorn. 


\section{Dedication}

This thesis is dedicated to my parents, Günter and Angela Hussenoeder. It is their unfailing love and guidance that have helped me most in achieving the goals I have set over the past several years. 


\section{Contents}

1 Introduction $\quad 17$

1.1 Background and Motivation ... . . . . . . . . 17

1.2 Thesis Overview . . . . . . . . . . . . . . . . . 20

2 Direct inversion of potential fields from an uneven track with application to the Mid-Atlantic Ridge 23

2.1 Introduction . . . . . . . . . . . . . . . . 23

2.2 Theory . . . . . . . . . . . . . . . . . . . 25

2.3 Discussion . . . . . . . . . . . . . . . . . . . . . 30

2.4 Conclusions . . . . . . . . . . . . . . . . . . . . . . . 32

2.5 Acknowledgments . . . . . . . . . . . . . . . 33

3 Near-bottom magnetic survey of the Mid-Atlantic Ridge axis, $24^{\circ}-$ $24^{\circ} 40^{\circ} \mathrm{N}$ : Implications for crustal accretion at slow spreading ridges 34

3.1 Introduction . . . . . . . . . . . . . . 35

3.1.1 Geologic Setting . . . . . . . . . . . . . . 38

3.2 Data Collection and Analysis . . . . . . . . . . . . 39

3.3 Results.......................... . . 43

3.3.1 Profile 1: Segment $2\left(24^{\circ} 34^{\prime} N\right) \ldots . . . . . . .44$

3.3.2 Profile 2: North End of Segment $1\left(24^{\circ} 21^{\prime} N\right)$. . . . . . 47

3.3.3 Profile 3: South Central Part of Segment $1\left(24^{\circ} 02^{\prime} \mathrm{N}\right)$. . . . 50 
3.3.4 Profile 4: The Nodal Deep and Inside Corner High $\left(23^{\circ} 54^{\prime} \mathrm{N}\right) \quad 52$

3.4 Discussion . . . . . . . . . . . . . . . . . 52

3.4.1 The Central Anomaly Magnetic High . . . . . . . . . . . . 54

3.4.2 Axial Valley Wall Magnetization Lows . . . . . . . . . . . . . 62

3.5 Conclusions . . . . . . . . . . . . . . . . 67

3.6 Acknowledgments . . . . . . . . . . . . . . . . 69

4 Seismic analysis of the axial magma chamber reflector along the southern East Pacific Rise from conventional reflection profiling $\quad 70$

4.1 Introduction . . . . . . . . . . . . . . . . . 71

4.2 Data Collection and Analysis . . . . . . . . . . . 73

4.3 Data . . . . . . . . . . . . . . . . . . . 79

4.4 Modeling and Results . . . . . . . . . . . . . . 83

4.4.1 Model Parameters ................ 85

4.4.2 Evidence for a Thin Magma Sill: Waveform Modeling of the Near-Offset Trace . . . . . . . . . . . . 87

4.4.3 Evidence for Nonzero Shear Wave Velocities: Amplitude Versus Slowness Analysis . . . . . . . . . . . . . . 92

4.5 Discussion . . . . . . . . . . . . . . . . . . . . . . 98

4.6 Conclusions . . . . . . . . . . . . . . . . . . 103

4.7 Acknowledgments . . . . . . . . . . . . . . . . . 104

5 Fine-scale seismic structure of young upper oceanic crust at $17^{\circ} 20^{\prime} \mathrm{S}$ on the fast spreading East Pacific Rise $\quad 105$

5.1 Introduction . . . . . . . . . . . . . . . 105

5.1.1 The Study Area . . . . . . . . . . . . . . . 109

5.2 Data Collection and Analysis ... . . . . . . . . . . 111

5.3 Results . . . . . . . . . . . . . . . . . 117

5.3.1 Along-Axis Profile: SEPR17 . . . . . . . . . . . . . 117 
$5.3 .23 \mathrm{~km}$ Off-Axis: SEPR14 \& SEPR15 . . . . . . . . . . . . 123

$5.3 .36 \mathrm{~km}$ Off-Axis: SEPR13 \& SEPR16 . . . . . . . . . . 126

5.4 Discussion . . . . . . . . . . . . . . . . . . . 135

5.4.1 Comparison with Previous Results and Methods . . . . . . 137

5.4 .2 Aging of Extrusive Crust . . . . . . . . . . . . . . 145

5.4 .3 Geological Interpretation . . . . . . . . . . . . . . . 149

5.5 Summary and Conclusions . . . . . . . . . . . . . 158

6 Upper crustal seismic structure of the slow spreading Mid-Atlantic Ridge, $35^{\circ} \mathrm{N}$ : Constraints on volcanic emplacement processes $\quad 160$

6.1 Introduction . . . . . . . . . . . . . . . . . . . . . 160

6.1 .1 The Study Area . . . . . . . . . . . . . . . . . . . 164

6.2 Data Collection and Analysis . . . . . . . . . . . . 167

6.3 Results . . . . . . . . . . . . . . . . . 176

6.3 .1 Velocity Models . . . . . . . . . . . . . . . . 176

6.3 .2 Seismic Profiles . . . . . . . . . . . . . . . 186

6.4 Discussion . . . . . . . . . . . . . . . . . . 193

6.4.1 Construction of the Upper Crust . . . . . . . . 196

6.4.2 Maturation and Modification of the Upper Crust . . . . . 200

6.5 Conclusions . . . . . . . . . . . . . . . . . 206

7 Conclusions 208

7.1 Synthesis of Results . . . . . . . . . . . . . . . . 208

7.2 Future Work . . . . . . . . . . . . . . . . 210

A The Genetic Algorithm 213

A.1 Model Parameterization . . . . . . . . . . . . . . . 215

A.2 Fitness Calculation . . . . . . . . . . . 216

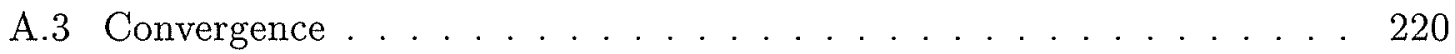

A.4 Error Analysis . . . . . . . . . . . . . . . . . . . . 224 


\section{List of Figures}

$1-1 \quad$ Overview of Study Sites $\ldots \ldots \ldots \ldots$

2-1 Approximate equivalence between geometries . . . . . . . . . . 25

2-2 Forward model using a draped survey geometry . . . . . . . . . . . . 29

2-3 Deep-tow magnetic profile across the $\operatorname{MAR}\left(24^{\circ} 20^{\prime} \mathrm{N}\right) \ldots \ldots$

3-1 Map of the western Kane RTI (study area) . . . . . . . . . . . . 37

3-2 Sea surface magnetic field anomaly map . . . . . . . . . . . . . . 40

3-3 Seafloor magnetization map from sea surface data . . . . . . . . . . . 44

3-4 Comparison of sea surface and deep tow magnetization profiles . . . . 45

3-5a Deep tow magnetic profile crossing segment 2 at $24^{\circ} 34^{\prime} \mathrm{N} \ldots \ldots$

3-5b Profile crossing the north end of segment 1 at $24^{\circ} 21^{\prime} \mathrm{N} \ldots . . . .49$

$3-5 \mathrm{c}$ Profile crossing the south central part of segment 1 at $24^{\circ} 02^{\prime} \mathrm{N}$. . . 51

3-5d Profile crossing the nodal deep and inside corner high at $23^{\circ} 54^{\prime} \mathrm{N}$. . 53

3-6a Forward model of the profile crossing segment $2 \ldots \ldots 5$

$3-6 \mathrm{~b}$ Profile at the north end of segment $1 \ldots \ldots$. . . . . . . 56

$3-6$ c Profile at the south central part of segment 1 . . . . . . . . 57

3-6d Profile crossing the nodal deep and inside corner high . . . . . . . 58

3-7 A sequential depiction of the cyclic faulting model . . . . . . . . 66

4-1 Map of the southern East Pacific Rise . . . . . . . . . . . . . . . . 74

4-2 Analysis of the 2-D+ $\tau-p$ transform method . . . . . . . . . 77

4-3 Constant offset and common midpoint stacks of the data . . . . . 80 
4-4 $\quad \tau-p$ transformed data and melt lens AVO curves . . . . . . . . . . . 81

4-5 Depiction of the model parameter space . . . . . . . . . . . . . 84

4-6 Wide-aperture profile from $14^{\circ} 10^{\prime} \mathrm{S}$ along the EPR . . . . . . . 86

4-7a Waveform modeling results for SEPR03-19'36'S . . . . . . . . . . 88

4-7b Waveform modeling results for SEPR09-17 $33^{\prime} \mathrm{S} \ldots \ldots$. . . . . . . . 89

4-7c Waveform modeling results for NEPR41-9 $39^{\prime} \mathrm{N} \ldots . . . . . .90$

4-8 Effect of melt lens thickness, $V_{p}$, and $V_{s}$ on AMC amplitude . . . 93

4-9a Amplitude vs. Slowness modeling results for SEPR03-19³6'S . . . . 95

4-9b Amplitude vs. Slowness modeling results for SEPR09-17³3'S . . . . 96

4-9c Amplitude vs. Slowness modeling results for NEPR41-9 $39^{\prime} \mathrm{N} \quad \ldots \quad . \quad 97$

4-10 Seismic velocity vs. temperature and/or crystal content . . . . . . 99

4-11 Sill thickness, $V_{p}$, and $V_{s}$ for the four southern EPR study sites . . . 101

5-1 Map of the $17^{\circ} 20^{\prime} \mathrm{S}$ study area, EPR . . . . . . . . . . . 110

5-2 Representative data supergathers . . . . . . . . . . . . . 113

5-3 Simple velocity model of upper crust, ray diagram, \& travel times . . 114

5-4 Velocity cross section of along-axis profile SEPR17 . . . . . . . . 120

5-5 GA waveform inversion results for SEPR17 supergather 14 . . . . 120

5-6 Velocity cross section of profile SEPR14, $3 \mathrm{~km}$ west of rise axis . . . . 124

5-7 GA waveform inversion results for SEPR14 supergather 3 . . . . . 126

5-8 Velocity cross section of profile SEPR15, $3 \mathrm{~km}$ east of rise axis . . . . 127

5-9 GA waveform inversion results for SEPR15 supergather 10 . . . . . 128

5-10 Velocity cross section of profile SEPR13, $6 \mathrm{~km}$ west of rise axis . . . . 130

5-11 GA waveform inversion results for SEPR13 supergather 12 . . . . . 131

5-12 Velocity cross section of profile SEPR16, $6 \mathrm{~km}$ east of rise axis . . . . 132

5-13 GA waveform inversion results for SEPR16 supergather 17 . . . . 133

5-14 Contour map of extrusive velocity . . . . . . . . . . . . 134

5-15 Contour map of layer 2A thickness . . . . . . . . . . . . . . 136

$5-16$ Velocity analysis from four locations along the EPR . . . . . . . 138 
5-17 Migrated seismic sections of along-axis profile, SEPR17 . . . . . . . 140

5-18 Migrated seismic sections of off-axis profile, SEPR16 . . . . . . . . 141

5-19 Migrated seismic section of cross-axis profile, SEPR27 . . . . . . . . 144

5-20 Extrusive velocities vs. crustal age at fast spreading ridges . . . . . 148

5-21 Cartoon cross-section of the EPR . . . . . . . . . . 157

6-1 Map of segment OH-1 on the MAR (study area) . . . . . . . . 165

6-2 Bathymetric profiles along MCS lines G, H, I \& L . . . . . . . . 168

6-3 Data supergathers from MCS lines G, H \& I . . . . . . . . . . . 169

6-4 Five data supergathers from MCS line L . . . . . . . . . . . . 171

6-5 Original and attenuated source waveform for the MARBE survey . . 175

6-6 Mean final velocity models for line G study sites . . . . . . . . . . 179

6-7 GA waveform inversion results for supergather G2 . . . . . . . 180

6-8 Mean final velocity models for line $\mathrm{H}$ study sites . . . . . . . . . . 181

6-9 GA waveform inversion results for supergather H1 . . . . . . . . . 182

6-10 Mean final velocity models for line I study sites . . . . . . . . . 183

6-11 GA waveform inversion results for supergather I1 . . . . . . . . 184

6-12 Mean final velocity models for line L study sites . . . . . . . . . 185

6-13 GA waveform inversion results for supergather L1 . . . . . . 186

6-14 True \& RMS velocity vs. travel time for each study site . . . . . . . 188

6-15 Migrated seismic sections of lines G \& H . . . . . . . . . . . . 189

6-15 (continued) Migrated seismic sections of lines I \& L . . . . . . . 190

6-16 Hand-modeled supergather from line H . . . . . . . . . . . . . . 191

6-17 Upper crustal velocity structure at different spreading rates . . . . . 195

6-18 Extrusive velocity vs. crustal age at slow spreading ridges . . . . . 202

A-1 Flow diagram for applying the GA to seismic waveform inversion . . . 214

A-2 Progression of a GA run on synthetic data . . . . . . . . . . 221 


\section{List of Tables}

3.1 Corner Wavelengths of Filters . . . . . . . . . . . . . 42

4.1 Summary of Results . . . . . . . . . . . . . . . . 75

5.1 Summary of Inversion Results . . . . . . . . . . . . . 118

6.1 Variable Parameter Settings for the Genetic Algorithm ${ }^{a} \ldots \ldots$. . . 173

6.2 Summary of Inversion Results . . . . . . . . . . . . . . 177

A.1 Tested and Applied ${ }^{a}$ Parameter Settings for the Genetic Algorithm . 217 


\section{Chapter 1}

\section{Introduction}

\subsection{Background and Motivation}

One of the underlying tenets of plate tectonic theory is that the network of midocean ridges that circles the globe marks the location of volcanically active spreading centers, which are associated with the rising limbs of mantle convection cells within a dynamically cooling earth. Although nearly all spreading centers generate crust of the same average thickness (6-7 km [e.g., Raitt, 1963; White et al., 1992]), geophysical studies have shown that the variable thermal state of the underlying mantle and wide range of spreading rates $(10-160 \mathrm{~km} / \mathrm{Myr}$ full-rate [DeMets et al., 1990]) produce a variety of mid-ocean ridge morphologies that exhibit different styles of melt generation and crustal accretion [Macdonald, 1986, 1989; Solomon and Toomey, 1992; Forsyth, 1992; Sparks and Parmentier, 1994]. Because of the numerous studies performed there, the slow spreading northern Mid-Atlantic Ridge (MAR) and the fast spreading southern East Pacific Rise (EPR) have become the archetypes of crustal formation at either end of the spreading rate spectrum. The linear, rise axis morphology of the EPR is thought to be controlled by two-dimensional, plate-driven mantle flow, whereas the more tectonically dominated rift valley morphology of the MAR is associated with a less vigorous, three-dimensional pattern of buoyant mantle 
upwelling [Whitehead et al., 1984; Parmentier and Phipps Morgan, 1990; Lin and Phipps Morgan, 1992]. This difference in mantle flow pattern and melt generation is manifested at slow spreading rates by increased along-axis variability in crustal thickness [Sinha and Louden, 1983; Kuo and Forsyth, 1988; Lin et al., 1990; Tolstoy et al., 1993; Rommevaux et al., 1994; Detrick et al., 1995] and ridge depth [Sempéré et al., 1993; Thibaud et al., 1997], as well as the absence of any resolvable crustal magma bodies [e.g., Fowler, 1976, 1978; Toomey et al., 1988; Detrick et al., 1990].

Regardless of spreading rate, all mid-ocean ridges repeatedly erupt to form a sequence of lava flow units that overlie the solidified conduits (or dikes) that fed them. Together, these two lithologic sections form the upper oceanic crust, or layer 2 , as determined from seismic refraction studies [Raitt, 1963; Fox et al., 1973]. Not only is layer 2 a direct product of crustal construction at the ridge axis, it is a comprehensive geological record of past variations in volcanic, tectonic and hydrothermal processes.

Crustal evolution is a continuous and variable process that is most active in the near-ridge environment. Detailed examination of the upper crust through its early developmental stages is therefore crucial to understanding active mid-ocean ridge processes and how they compare in different spreading regimes. Dredge programs [e.g., Schilling et al., 1982; Langmuir et al., 1986], submersible dives [e.g., Ballard and Van Andel, 1977; Francheteau et al., 1990], ophiolite studies [e.g., Moores, 1982; Nicolas, 1989] and drill holes [e.g., Shipboard Scientific Party, 1979; Becker et al., 1988 ] yield important information about the composition and vertical structure of the upper crust, but are restricted to mature tectonic settings (sedimented or obducted) and/or limited in their spatial coverage. Swath mapping surveys [e.g., Smith and Cann, 1990; Searle et al., 1998] provided constraints on the lateral extent of volcanic processes, but are unable to resolve deeper structure.

Magnetic and seismic methods are excellent means of continuously measuring the geometry and internal structure of young upper oceanic crust. Because of their sensitivity to the extrusive section, these methods have greatly increased our under- 
standing of the relationship between mid-ocean ridge dynamics and shallow crustal structure. The concept of a thin, surficial layer (termed $2 \mathrm{~A}$, since it constitutes the top of layer 2) with distinct physical properties was independently developed by the magnetic and seismic communities in the early 1970's. Using magnetic profiles and heat flow measurements, Talwani et al. [1971] demonstrated the existence of a 400m-thick, highly magnetized and porous "layer $2 \mathrm{~A}$ ", which they interpreted as the pillow lava section. Houtz and Ewing [1976] later defined layer 2A as the low-velocity (3-4 km/s) layer within the uppermost kilometer of crust- a definition that has been refined as acquisition (e.g., navigation and recording technology) and analysis techniques (e.g., data processing and inversion) have improved. Today, layer 2A continues to be defined seismically as the surficial low-velocity interval, and is often (albeit arguably) associated with the erupted lava section. Beneath the steep velocity gradient at the base of layer $2 \mathrm{~A}$, the high velocities of layer $2 \mathrm{~B}$ are often treated as the sheeted dikes. Similarly, the extrusive section is thought to carry the bulk of the crustal magnetic signature, especially in the near-ridge environment. This magnetic source layer is often equated with seismic layer $2 \mathrm{~A}$. (The current state of knowledge regarding the magnetic and seismic properties of the upper crust is presented in the Introductions of Chapters 3 through 6.)

Since the sheeted dikes and overlying extrusives are created by volcanic processes, we can use their magnetic and seismic signature to address several questions about mid-ocean ridge volcanism. For example, how focused is melt delivery to the seafloor? How distributed is off-axis lava deposition? What is the total volume of extrusives produced? How do the answers to these questions compare from one spreading regime to another? Are there significant along-axis trends in volcanic accretion at the MAR? What are the characteristics of the magma sill at the fast spreading EPR, and how do they affect the eruptive process?

The rapid off-axis evolution of seismic and magnetic properties also provide constraints on the hydrothermal and tectonic modification of young oceanic crust. For 
example, to what degree does hydrothermal alteration affect the bulk properties of the upper crust? Is it less vigorous at slower spreading rates? Is it enhanced at specific levels in the crust? How do the differing tectonic regimes of fast and slow spreading centers affect the construction and preservation of the volcanic stratigraphy?

\subsection{Thesis Overview}

This thesis uses magnetic and seismic methods to examine the geometry and internal structure of the upper crust at the fast spreading East Pacific Rise and slow spreading Mid-Atlantic Ridge (Fig. 1-1). We exploit the sensitivity of these methods to shallow crustal structure by developing and applying new processing techniques that maximize their resolution capability. By understanding how the extrusive crust is built and modified in two end-member spreading regimes, we will gain a more global picture of shallow crustal processes at mid-ocean ridges.

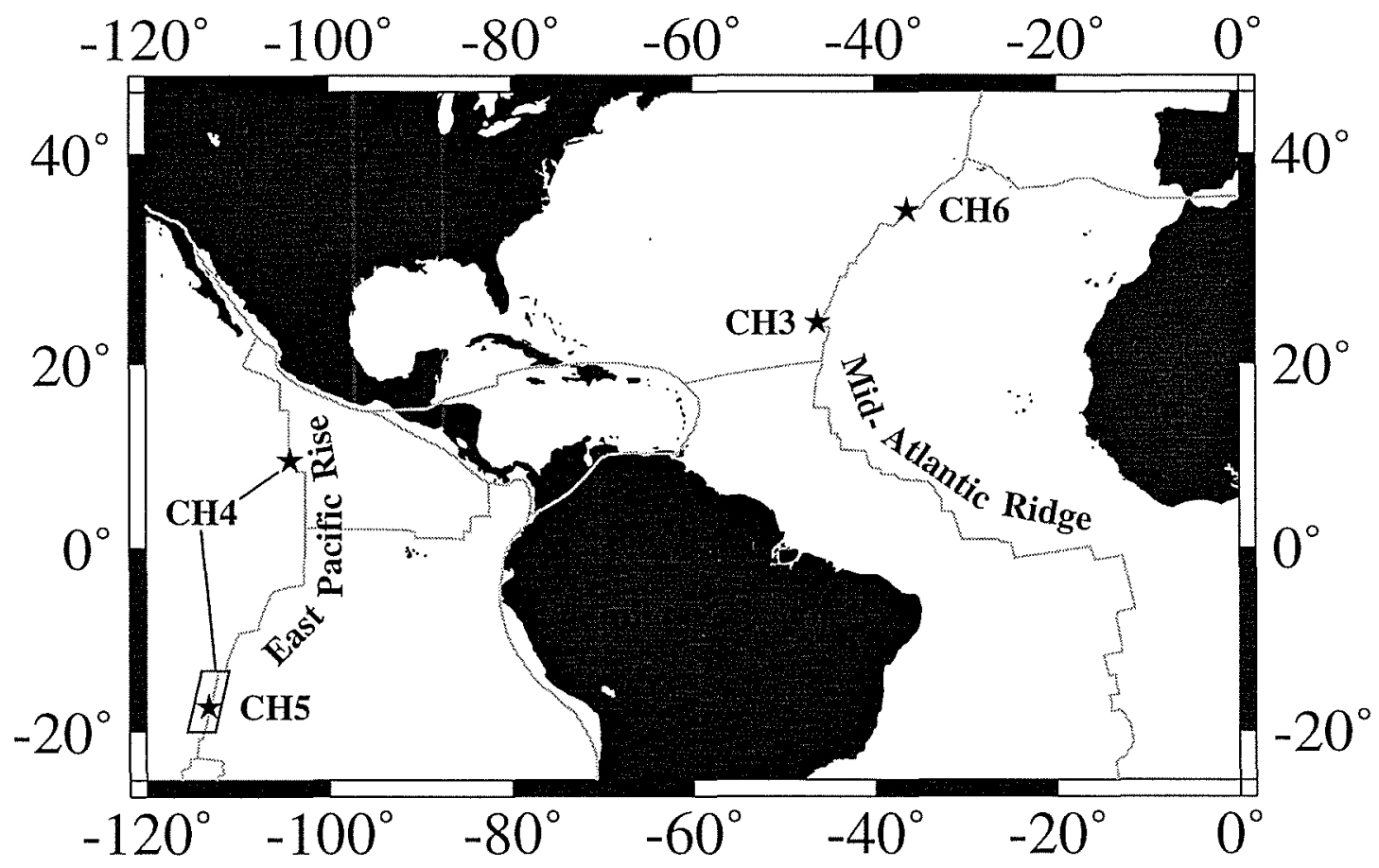

Figure 1-1: Overview of Study Sites 
In Chapter 2, we develop a potential field inversion technique that retains the full resolution offered by near-bottom magnetic surveys. By directly inverting the data from an uneven observation track, this method bypasses the (previously necessary) step of upward continuation to a level plane, thereby avoiding the associated loss of high-frequency information. In Chapter 3, we apply the method of Chapter 2, and more conventional inversion and forward modeling techniques, to near-bottom magnetic data from the Mid-Atlantic Ridge (Fig. 1-1) in order to study the role of magmatism and tectonic disruption in generating the variability of newly formed crust at slow spreading ridges.

We then shift our focus in Chapter 4 to the East Pacific Rise, where the dimensions and internal properties of the mid-crustal magma sill are studied at several locations (Fig. 1-1). Although volumetrically quite small, the magma sill is thought to play a key role in the availability and composition of melt erupted at the rise axis to form the upper crust [Sinton and Detrick, 1992]. Through a combination of waveform and amplitude modeling, we examine the size and molten state of this body and discuss the impact of these characteristics on the transport of melt within the volcanic cycle of eruption and replenishment.

Using the genetic algorithm-based velocity inversion technique developed in Appendix A, the next two chapters examine the seismic structure of layer 2 (the product of crustal magma bodies). Chapter 5 focuses on the superfast spreading southern East Pacific Rise at $17^{\circ} 20^{\prime}$ S, while Chapter 6 deals with a magmatically robust segment of the slow spreading Mid-Atlantic Ridge (Fig. 1-1). In examining the evolution of the upper crust, these two chapters provide new constraints on volcanic construction, hydrothermal alteration and tectonic disruption, as well as how they vary with spreading rate. This is especially true for the Mid-Atlantic Ridge, where the extreme bathymetry and complicated interplay of geological processes has concealed much about crustal formation at slow spreading rates.

Chapter 7 summarizes the major conclusions presented in Chapters 3 through 
6, discusses future applications of the methods developed in Chapter 2 and the Appendix, and highlights some possible approaches to the remaining outstanding questions regarding the construction and modification of upper oceanic crust.

Chapter 2 was previously published in Geophysical Research Letters [1995], and Chapters 3 and 4 were published in Journal of Geophysical Research [1996]. They have been modified only to conform to thesis format and to update references. The full citations for those papers appear in the Curriculum Vita and at the end of the corresponding chapters. Chapter 5 and the Appendix will be combined and submitted to Journal of Geophysical Research, as will Chapter 6. Graham Kent and Robert Detrick will be co-authors on both papers, while Alistair Harding will be a co-author on Chapter 5 only. 


\title{
Chapter 2
}

\section{Direct inversion of potential fields from}

\section{an uneven track with application to the}

Mid-Atlantic Ridge

\begin{abstract}
Current methods of potential field inversion require measurements to be reduced onto a level plane, resulting in a loss of resolution. This is especially true for draped surveys in areas of extreme topography. We examine a method that bypasses this limiting step by making use of an approximately equivalent geometry from which data are directly inverted. Corrections are applied to an initial solution to account for errors generated by the transformation of geometries. A numerical model and an example data set are used to explore this method's benefits and drawbacks in relation to the conventional approach of data reduction onto a level plane prior to inversion. We show that this direct inversion method is particularly better than the conventional approach at resolving fine-scale features, such as the narrow zone of crustal emplacement at the Mid-Atlantic Ridge and adjacent areas of tectonic disruption and magnetic source alteration.
\end{abstract}

\section{$2.1 \quad$ Introduction}

Most high resolution potential field surveys, such as near-bottom marine magnetics, acquire data along an uneven track. Since current inversion methods require measurements to be on a level plane, various techniques have been designed to reduce 
data onto such a plane. Direct reduction methods have been employed in the past, either using the Schwarz-Christoffel transformation [Parker and Klitgord, 1972] or an iterative equation in the frequency domain [e.g. Guspi, 1987] to solve for the field on a level plane. Indirect methods have also been developed which compute the field at a constant level from an equivalent source distribution [Hansen and Miyazaki, 1984; Pilkington and Urquhart, 1990]. They involve an intermediate step of computing equivalent sources, but are more stable than direct methods.

The most serious drawback to reducing data onto a level plane is that a loss of resolution is inevitable. Most reduction methods use a high-cut filter to prevent the amplification of noise, either recorded or generated by approximations, ensuring convergence of the solution, but resulting in a loss of spatial resolution [Schouten and McCamy, 1972]. Reduced data must often be upward continued to a level above the topography in order to perform the inversion. This causes further loss of resolution as the upward continuation filter irretrievably suppresses high-frequency information. The high-cut filter of the subsequent inversion must then suppress a larger range of high frequencies than would have been necessary if the data were closer to the sources, such as the original observations. This problem is particularly evident in draped surveys where, rather than make use of the high resolution offered by the proximity of the sources, one ultimately obtains a filtered approximation of a field that could have been measured directly by a constant altitude survey.

An inversion directly from the vehicle track would be quite advantageous. It would not suffer from such a loss of resolution, because the reduction of data onto a level plane, and associated high-cut filter, would be eliminated. In the following, we examine a technique for inverting potential fields directly from an uneven track. It is based on the equivalent source method of Pilkington and Urquhart [1990], but extends their method to the determination of crustal source distributions. 


\subsection{Theory}

Consider measurements taken during a variable altitude survey. For clarity, altitude and depth are measured relative to sea level, and flying height relative to surface topography. For any survey geometry, an approximately equivalent geometry exists that both maintains flying height variations and exhibits a level observation plane (Fig. 2-1). It is this equivalence that forms the basis of Pilkington \& Urquhart's [1990] approximate equivalence reduction technique in which an approximate source distribution is calculated by assuming the vehicle track to be level and topography to be a mirror image of the vehicle track. A reduced field is then calculated from this source distribution. They note that the integrated effect of these sources at an observation point varies as the geometry is changed, and that this effect is significant in cases of extreme topography.

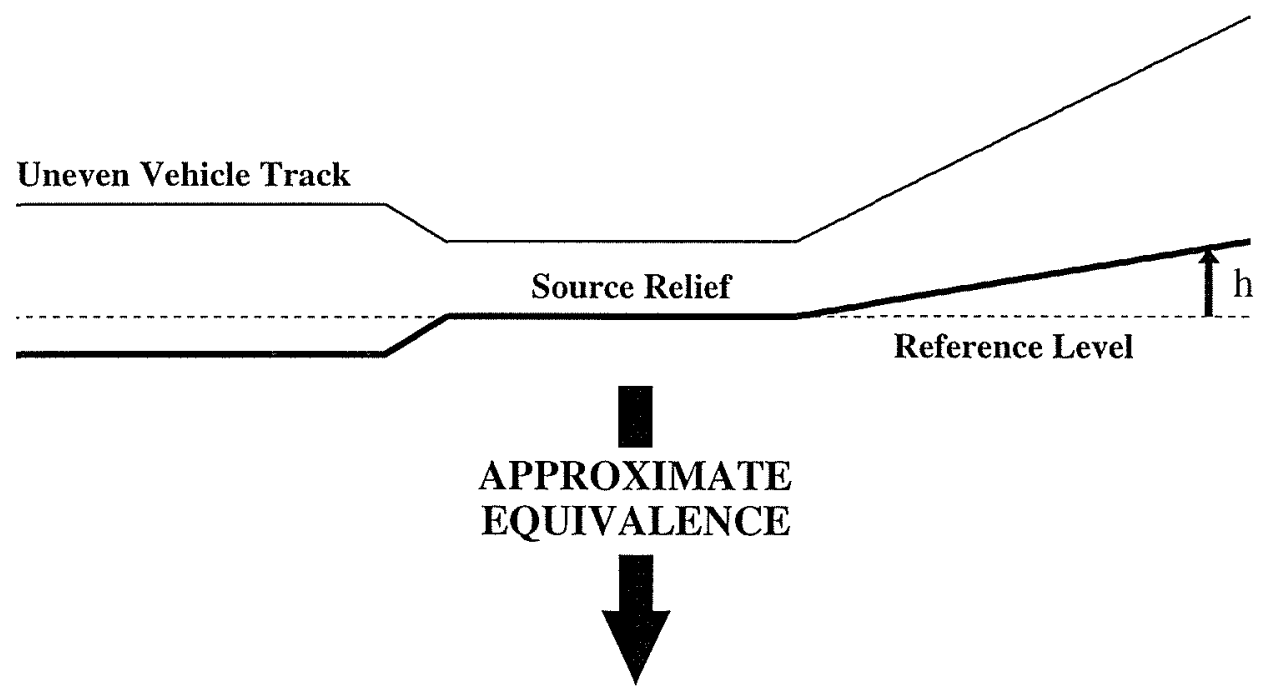

Equiv. Observation Plane

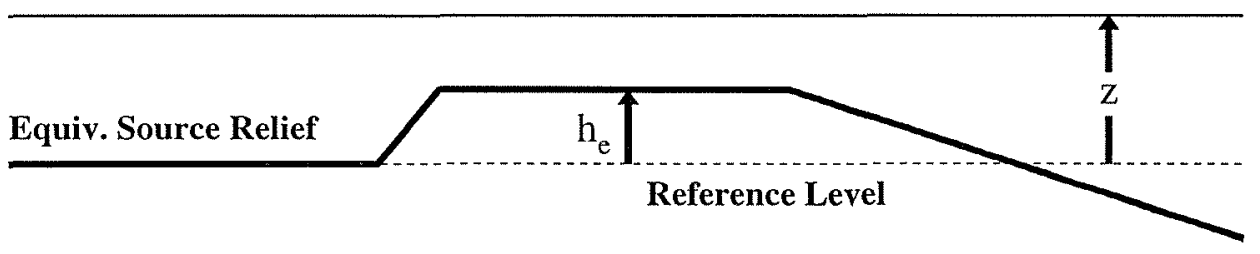

Figure 2-1: Approximate equivalence between geometries. In any survey, a corresponding geometry exists that maintains flying height variations and exhibits a level observation plane. The integrated effect of sources on an observation point varies as geometry changes. 
We make use of the above equivalence by solving for the magnetization on an approximation of the actual relief, not an imaginary surface. This precludes the need to reduce data onto a plane before inverting. To accomplish this, a mirror image of the altitude variations is added to both the topography and uneven vehicle track, thus maintaining flying height while transforming the uneven track to a constant level. This step does not affect the data. It merely generates a more tractable geometry (Fig. 2-1). The problem now reduces to solving for a source distribution, $J_{D I}$, that reproduces the observations in the approximately equivalent geometry. Assuming a constant thickness source layer in the magnetic case, a solution can be obtained by the successive approximation method of Parker and Huestis [1974]:

$$
\begin{aligned}
\mathcal{F}\left[J_{D I}(\mathbf{r})\right] & =\mathcal{F}[B(\mathbf{r})] C-\sum_{n=1}^{\infty} \frac{|\mathbf{k}|^{n}}{n !} \mathcal{F}\left[J_{D I}(\mathbf{r}) h_{e}^{n}(\mathbf{r})\right] \\
C & =e^{|\mathbf{k}| z} e^{i \theta}\left[2 \pi A\left(1-e^{-|\mathbf{k}| h_{o}}\right)\right]^{-1}
\end{aligned}
$$

$\mathcal{F}\left[J_{D I}\right]$ and $\mathcal{F}[B]$ are Fourier transforms of the magnetization and measured field respectively, $\mathbf{k}$ is the wavenumber array, $h_{e}$ is topographic deviation from a reference level in the equivalent geometry, $z$ is distance between this reference level and the equivalent observation plane, and $h_{o}$ is magnetic layer thickness (Fig. 2-1). $A$ is an amplitude factor and $\theta$ is a phase parameter, both dependent on magnetization direction and the ambient field [Schouten and McCamy, 1972].

Errors are generated by the inability of Equation 2.1 to correctly retrieve information at wavelengths equal to the bathymetric variation, since it inverts within a flattened equivalent of the true geometry. These errors becomes significant in cases of extreme topography, such as mid-ocean rift valleys, and in non-draped surveys, but they can be quantified and corrected. Our approach is to calculate the effect of the transformation between geometries upon $J_{D I}$ by computing the field due to a known magnetization and then applying the direct inversion. This provides us with 
a measure of the error in Equation 2.1.

The first step of the correction procedure is to calculate the field, $B_{t}$, that a known, $1 \mathrm{~A} / \mathrm{m}$ constant thickness crust generates on the true vehicle track in the original geometry. This is best accomplished analytically [Talwani and Heirtzler, 1964; Won and Bevis, 1987]. The direct inversion (Eq. 2.1) is then applied to this field:

$$
\mathcal{F}\left[J_{t}(\mathbf{r})\right]=\mathcal{F}\left[B_{t}(\mathbf{r})\right] C-\sum_{n=1}^{\infty} \frac{|\mathbf{k}|^{n}}{n !} \mathcal{F}\left[J_{t}(\mathbf{r}) h_{e}^{n}(\mathbf{r})\right]
$$

Since the effects of topographic variation on an inversion solution scale linearly with magnetization, Equation 2.2 represents the per $\mathrm{A} / \mathrm{m}$ effect of not only switching geometries but also inverting within the equivalent geometry. However, the inversion is not a source of error, merely a source of nonuniqueness. Its effect must be subtracted from Equation 2.2 to obtain the error in $J_{D I}$ solely due to the rearrangement in geometry. We quantify the effect of the inversion alone by first computing the field, $B_{e}$, that a known, $1 \mathrm{~A} / \mathrm{m}$ constant thickness crust generates on the observation plane in the equivalent geometry [Parker, 1972]:

$$
\mathcal{F}\left[B_{e}(\mathbf{r})\right]=C^{-1} \sum_{n=0}^{\infty} \frac{|\mathbf{k}|^{n}}{n !} \mathcal{F}\left[1 \cdot h_{e}^{n}(\mathbf{r})\right]
$$

One can then directly invert $B_{e}$ :

$$
\mathcal{F}\left[J_{e}(\mathbf{r})\right]=\mathcal{F}\left[B_{e}(\mathbf{r})\right] C-\sum_{n=1}^{\infty} \frac{|\mathbf{k}|^{n}}{n !} \mathcal{F}\left[J_{e}(\mathbf{r}) h_{e}^{n}(\mathbf{r})\right]
$$

If the true magnetization, $J_{T}$, responsible for the measured field were known, a correction to $J_{D I}$ could be calculated by multiplying the per $\mathrm{A} / \mathrm{m}$ error, $J_{t}-J_{e}$, by $J_{T}$. The resulting values could then be subtracted from $J_{D I}$ to yield the true magnetization:

$$
J_{T}(\mathbf{r})=J_{D I}(\mathbf{r})-\left[J_{t}(\mathbf{r})-J_{e}(\mathbf{r})\right] \cdot J_{T}(\mathbf{r})
$$


which can be rewritten as,

$$
J_{T}(\mathbf{r})=\frac{J_{D I}(\mathbf{r})}{1+J_{t}(\mathbf{r})-J_{e}(\mathbf{r})}
$$

One must be aware that the combined effects of filtering and the inherent nonuniqueness of inversions prevent the correction term from recovering the true magnetization, $J_{T}$, perfectly.

In surveys where the total topographic variation exceeds the flying height, inclusion of the correction distribution, particularly $J_{t}$, is necessary. It should also be included in cases where flying height varies by a factor of two or more, since $J_{e}$ is non-zero in geometries that deviate from being perfectly draped. As we have shown, the calculations involved in determining a correction distribution are quite simple, and can be computed in a time comparable to the reduction step in the conventional approach. These equations are valid in both two and three dimensions, and can be applied to gravity data by omitting the layer-thickness and phase terms and replacing the magnetization and field vectors with their gravitational counterparts [Parker, 1972].

Let us examine this method in the form of a forward model (Fig. 2-2). The geometry is of a draped survey performed by the RSS Charles Darwin towing the TOBI (Towed Ocean Bottom Instrument) system across the rift valley of the MidAtlantic Ridge north of the Kane transform in 1991 [Lawson et al., 1996]. A magnetic reversal pattern, in accordance with appropriate spreading rates is imposed on a $500 \mathrm{~m}$ thick layer, as is an area of strong magnetization at the neovolcanic axis (central anomaly magnetic high-CAMH). The field on the vehicle track is calculated analytically [Talwani and Heirtzler, 1964; Won and Bevis, 1987], and inverted using two methods: the conventional method of reduction onto a plane followed by inversion, and that of direct inversion. The method chosen to reduce the data onto a plane is that of Guspi [1987], whereby a reduced field is obtained directly from a Fourier-based iterative scheme much like Equation 2.1. Iterations are carried out to twenty terms, 

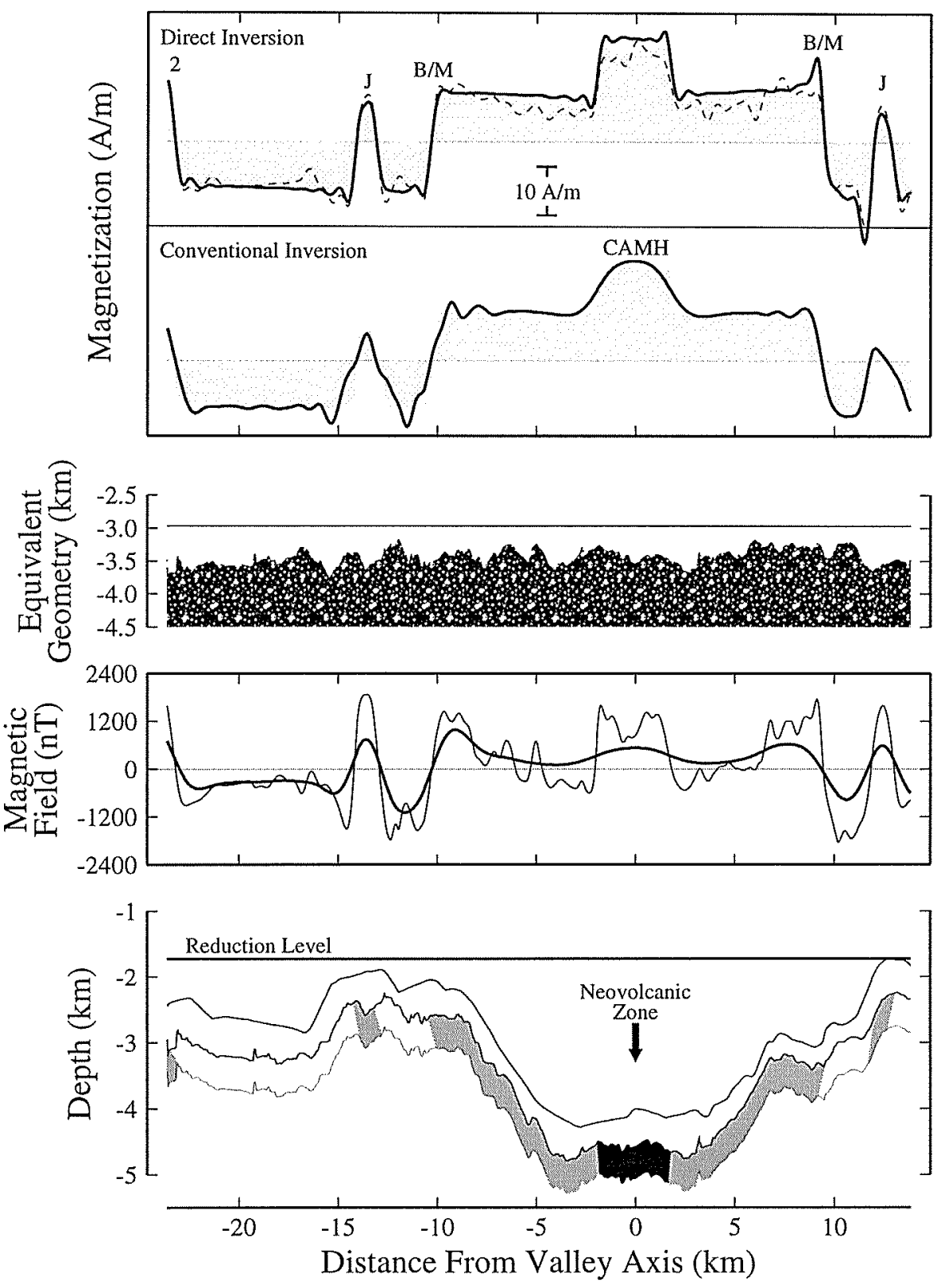

Figure 2-2: Forward model using draped survey geometry: (bottom) Original geometry. Magnetic reversal pattern based on a constant spreading rate, with gray areas $10 \mathrm{~A} / \mathrm{m}$, white $-10 \mathrm{~A} / \mathrm{m}$, and black $20 \mathrm{~A} / \mathrm{m}$. The reduction plane is at the minimum observation depth. (lower) The observed field (light curve), obtained analytically [Won and Bevis, 1987], is reduced (dark curve) by carrying Guspi [1987] to 20 iterations with a $1.3 \mathrm{~km}$ wavelength high-cut filter. (upper) Equivalent geometry used in the direct inversion. (top) Complete direct, $J_{T}$, conventional (dark curves), and uncorrected direct inversion (dashed), $J_{D I}$, are shown with input magnetization superimposed. Inversions are taken to 20 iterations with a $700 \mathrm{~m}$ corner wavelength filter. Bold letters locate the Brunhes/Matuyama $(B / M)$ polarity transition and Jaramillo $(J)$ epoch. 
and cosine-tapered, high-cut filters are used in the reduction and both inversions to prevent gross amplification of frequencies with a low signal-to-noise ratio (see Fig. 2-2 for filter values).

Both methods reproduce the input magnetization to varying degrees (Fig. 2-2). However, the long corner wavelength $(1.3 \mathrm{~km})$ of the reduction step's high-cut filter generates an overly smooth solution in the conventional method. Both the complete, $J_{T}$, and uncorrected direct inversion, $J_{D I}$, recover the sharpness and true location of the magnetization contrasts precisely because they do not suffer from the irretrievable loss of such a large bandwidth of information. The only loss of information is caused by the high-cut inversion filter, which is also used in the conventional method's inversion step. Application of the correction distribution improves the solution greatly, and is necessary, since flying height varies by a factor $>3$ and total bathymetric variation greatly exceeds flying height. The only locations where an excellent fit does not occur are where polarity transitions lie within a steeply sloping region. At those locations, a combination of the Gibbs phenomenon and extreme topography generates additional error that is not included in the correction distribution.

\subsection{Discussion}

We now apply this method to a real magnetic survey. Figure $2-3$ shows the same geometry, but includes the magnetic field actually measured during the survey. In the previous model (Fig. 2-2), the conventional method was less able to resolve the crustal magnetization structure of the neovolcanic zone and large amplitude transitions. A similar loss of resolution is seen in this figure over the axial valley, where the smooth, broad magnetization high is a substantially filtered version of the original field (Fig. 2-3). This loss of information must be carefully considered, because accurate measurements of the valley floor are crucial to an understanding of crustal formation and rift valley dynamics. 


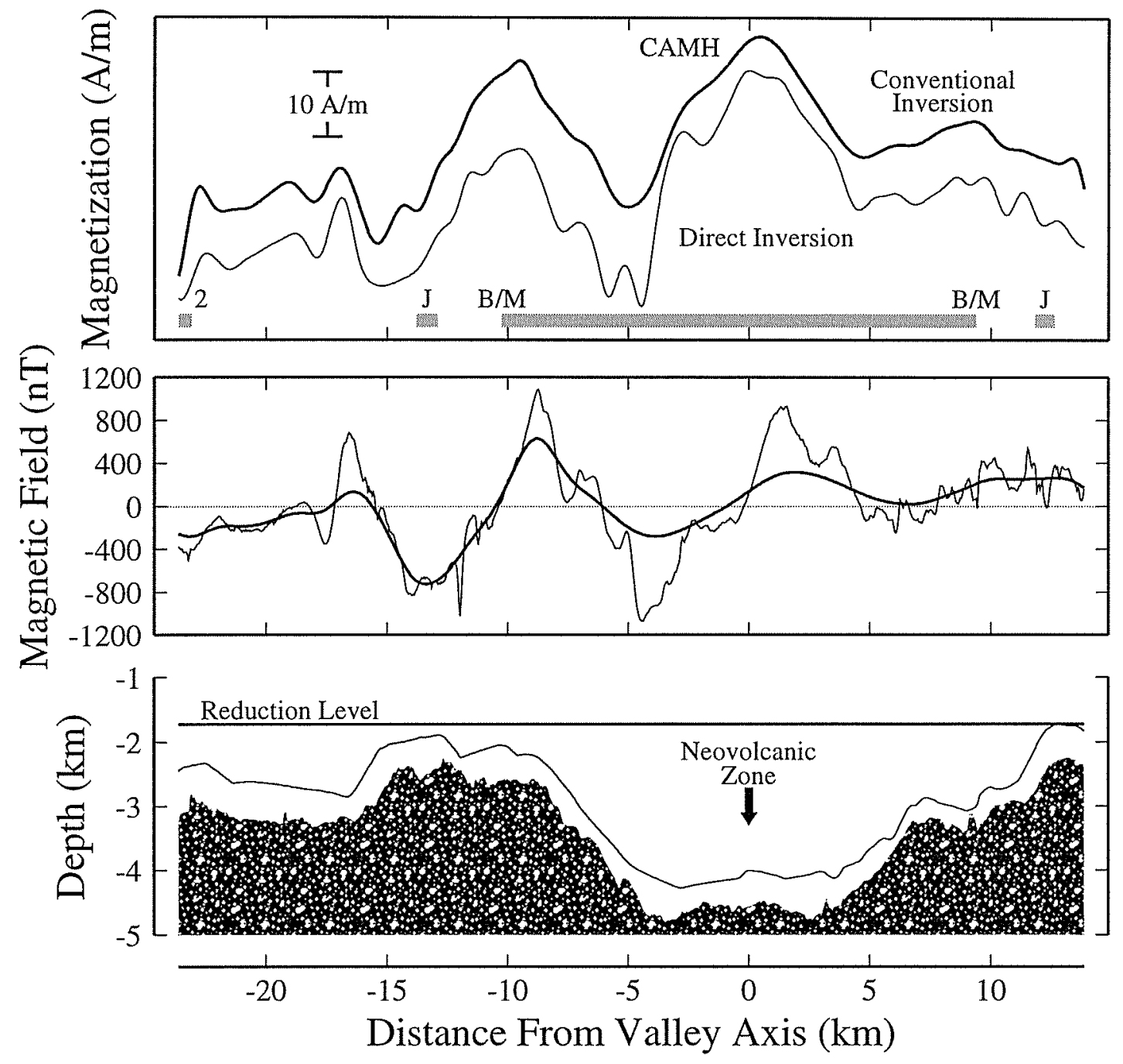

Figure 2-3: Deep-tow magnetic profile across the MAR $\left(24^{\circ} 20^{\prime} \mathrm{N}\right)$ [Lawson et al., 1996]: Panels arranged as in Figure 2-2. The neovolcanic zone coincides with the magnetic high in both solutions. Note the large continuation distance and resultant loss of resolution over the valley floor. The observed field is reduced using Guspi [1987] with a $1.3 \mathrm{~km}$ wavelength high-cut filter. Both methods use a $1.1 \mathrm{~km}$ corner wavelength inversion filter, and are taken to 20 iterations. The inversion assumes a $500 \mathrm{~m}$ thick source layer and geocentric dipole direction for the magnetization. Dark bars below the magnetization curves indicate normal polarity intervals, based on the constant spreading rate of Figure 2-2. 
The direct inversion provides improved resolution over conventional methods. For example, the CAMH is more narrowly defined by the direct inversion, suggesting a more precise location for the neovolcanic axis and focussed crustal accretion. We also resolve a rapid decay in magnetization with age. While not as rapid as at the East Pacific Rise [see Gee and Kent, 1994], this decay rate, with an exponential time constant of 0.1-0.2 m.y., is much faster than the $0.5 \mathrm{~m} . \mathrm{y}$. value inferred from previous studies at slow-spreading ridges [Johnson and Atwater, 1977; Macdonald, 1977]. Both magnetization solutions show deep lows corresponding to the rift valley walls, most notably to the west. These lows are within the Brunhes positive polarity epoch, and are interpreted to be areas of source destruction due to brittle deformation and low temperature alteration of the magnetic source. Note, however, the high magnetization outside the inner valley walls. This structure may indicate a tectonic process whereby large, unaltered blocks of crust are lifted out of the rift valley by adjacent zones of faulting. These observations of fine-scale magnetic structure show that, while emplacement of crust at slow-spreading ridges is highly focussed (in contrast to previous models [e.g. Schouten and Denham, 1979], subsequent tectonic disruption and alteration at the rift valley walls degrade the crustal magnetization signal and may be the primary reason for Atlantic magnetic anomaly variability.

\subsection{Conclusions}

The practice of reducing potential field data onto a level plane prior to inverting for the source distribution has prevented scientists from making full use of the increased resolution offered by draped surveys. We have shown that, in bypassing this limiting reduction step, the direct inversion method is better able to resolve crustal magnetic structure. This has been demonstrated in a forward model and an example data set. In cases of extreme topography and in geometries that significantly deviate from a draped survey, a correction to the direct inversion is central to computing an 
accurate solution. However, the simplicity of the direct inversion becomes manifest in less extreme geometries, where the correction term may be eliminated.

\subsection{Acknowledgments}

We thank R.C. Searle for permission to process the magnetic data in this study, as well as M. Pilkington and G. Acton for their helpful comments. Funding was provided by an ONR fellowship (S. Hussenoeder), and NSF grant OCE-9204121 (M. Tivey \& H. Schouten). Woods Hole Oceanographic Institution contribution 9083.

Hussenoeder, S. A., M. A. Tivey, and H. Schouten, Direct inversion of potential fields from an uneven track with application to the Mid-Atlantic Ridge, Geophys. Res. Lett., 22, 3131-3134, 1995. Copyright by the American Geophysical Union. Reprinted with permission. 


\title{
Chapter 3
}

\section{Near-bottom magnetic survey of the}

\section{Mid-Atlantic Ridge axis, $24^{\circ}-24^{\circ} 40^{\prime} \mathrm{N}$ :}

\section{Implications for crustal accretion at}

\author{
slow spreading ridges
}

\begin{abstract}
Near-bottom magnetic field measurements provide increased spatial resolution over sea surface magnetic data and allow a detailed analysis of the accretionary and tectonic processes at work in a slow spreading environment. Through the use of magnetic inversion methods and forward modeling, we investigate the fine-scale magnetic structure of young oceanic crust along four near-bottom profiles that cross the two bathymetric segments immediately north of the Kane transform on the Mid-Atlantic Ridge. Our results show the presence of a narrow, peaked central anomaly magnetic high (CAMH) located over the zone of most recent volcanism. We hypothesize that the pattern of high magnetization at segment ends and relatively low values at their centers is largely the result of increased iron and titanium content away from segment centers. Magnetization lows, which are not observed in the sea surface data, flank the CAMH and are associated with the axial valley walls. These lows may locate areas of intense magnetic source layer disruption. Blocks of relatively high magnetization found outside the valley walls suggest a cyclic faulting process, whereby discrete blocks of relatively unaltered crust are transported out of the rift valley by adjacent zones of faulting. These observations of fine-scale magnetic structure show that while the emplacement of crust at slow spreading ridges is highly focused, subsequent tectonic disruption and alteration at the rift valley walls degrades the crustal magnetization signal and may be the primary reason for Atlantic magnetic anomaly variability.
\end{abstract}




\subsection{Introduction}

Sea surface magnetics has been an integral part of geophysical studies for decades. At the dawn of plate tectonic theory, correct identification of the origin of seafloor anomalies provided crucial evidence for seafloor spreading [Vine and Matthews, 1963]. Magnetic anomalies have allowed us to determine spreading rates at divergent margins, perform plate reconstructions, and study numerous aspects of the volcano-tectonic history of oceanic crust. Shipboard magnetics has also shown that the magnetic anomaly pattern at the slow spreading Mid-Atlantic Ridge (MAR) is not as welldefined as at fast spreading ridges [e.g., Vogt, 1986]. The increased variability of magnetic anomalies at slow spreading ridges has motivated the need for geophysical techniques offering higher spatial resolution.

The greater spatial resolution offered by near-bottom magnetics [e.g., Macdonald, 1977; Tivey and Johnson, 1987] has allowed it to become a complement to bottom sampling and acoustic imaging techniques for exploring the fine-scale structure of the seafloor. Recent observations also provide evidence that the accretionary process at slow spreading ridges is more three-dimensional as a result of discrete, episodic emplacement [Smith and Cann, 1990] and active tectonic disruption of young oceanic crust. The extent that episodic magmatism and tectonic alteration participate in generating the magnetic variability of newly formed crust is a fundamental question concerning mid-ocean ridge processes, one that near-bottom magnetics is ideally suited to address.

Extensive studies of the Mid-Atlantic Ridge, such as the French-American Ocean Underwater Study (FAMOUS) [e.g., Johnson and Atwater, 1977; Macdonald, 1977] and Mid-Atlantic Ridge North of Kane (MARNOK) [e.g., Purdy et al., 1990; Smith and Cann, 1990; Sempéré et al., 1990, 1993], have yielded much information on the emplacement and modification of oceanic crust. Yet, many questions concerning controls on the fine-scale magnetic structure of mid-ocean ridges remain unresolved. The most dominant magnetic feature within the rift valley is the central anomaly magnetic 
high (CAMH), which is thought to represent the most recent volcanic emplacement [Klitgord, 1976; Tivey and Johnson, 1987]. The accuracy with which the CAMH predicts the location of the neovolcanic zone (NVZ) in a slow spreading environment is not well known. The origin of along-axis variability in the CAMH is also highly speculative, and several models, including Curie isotherm depth and geochemical variations, have been invoked to explain this phenomenon. The cross-axis shape of the $\mathrm{CAMH}$ is thought to be governed by low-temperature alteration off-axis, but there is wide disagreement on the rate of magnetic decay this process generates [Johnson and Atwater, 1977; Macdonald, 1977; Gee and Kent, 1994].

In 1991, a near-bottom survey over the two spreading segments immediately north of the Kane transform on the MAR was conducted by the RSS Charles Darwin in order to precisely map their tectonic and volcanic structures. The survey included several near-bottom side-scan and magnetometer transects across the median valley using the Towed Ocean Bottom Instrument (TOBI) [Allerton et al., 1995] (Figure 31). Numerous dredges within the axial valley and ridge-transform intersection (RTI), and two Wide-Angle Seafloor Photography (WASP) [Kleinrock, 1992] camera tows across the valley floor were also performed [Lawson et al., 1996]. The near-bottom profiles lie within the MARNOK area and provide an excellent opportunity to study the fine-scale magnetic structure of ocean crust in relation to seafloor morphology and volcanism, as well as its correlation with sea surface measurements.

The goal of this study is twofold. First, through the use of new data analysis methods, and the high resolution offered by near-bottom measurements, we examine the effect of crustal emplacement and modification within the rift valley on intrasegment variability. We address several questions concerning the nature of, and controls on, the short-wavelength magnetic anomalies found over the MAR, thereby providing constraints on the structure and dynamics of the slow spreading ridge environment. Second, we place these magnetic data within the larger framework of sea surface measurements for a clearer understanding of not only cross-axis but also along-axis 


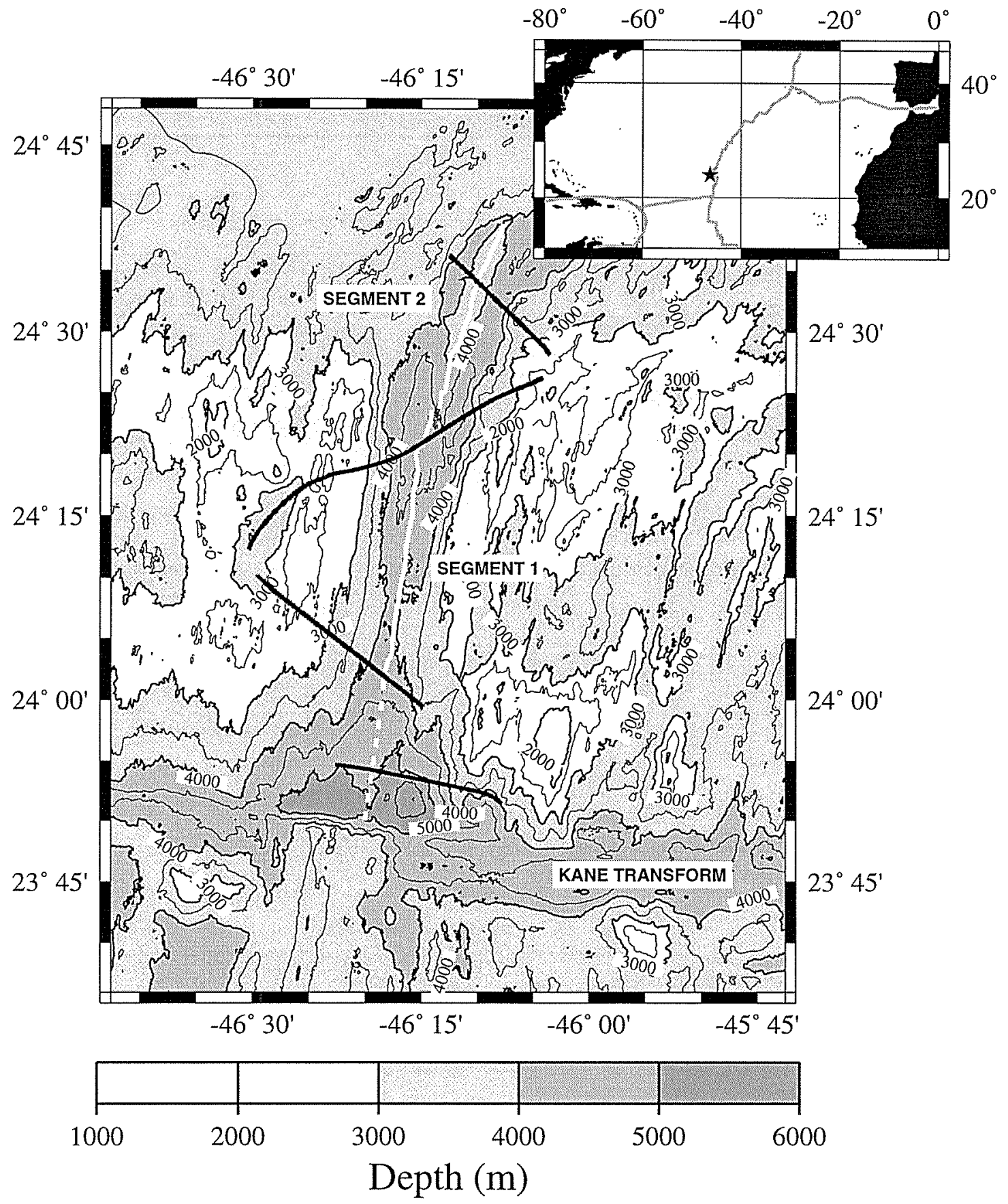

Figure 3-1: Bathymetric map of the ridge-transform intersection (RTI) and two spreading segments immediately north of the Kane transform [Fujimoto et al., 1994]. The two segments are separated by an area where the morphologic axis (white line [D. K. Smith, personal communication, 1995]) becomes indistinct. It is dashed where the axis has previously been difficult to locate, although a distinct NVZ does extend well into the RTI. Black lines mark the four near-bottom TOBI profiles obtained in 1991. 
magnetic variability. By answering some specific questions regarding the fine-scale magnetic structure of young oceanic crust, we begin to understand the origin of magnetic anomaly variability in a slow spreading environment.

\subsubsection{Geologic Setting}

Sea surface magnetic anomaly identification has determined that the ridge immediately north of the Kane transform has been spreading asymmetrically at an average half rate near $14 \mathrm{~mm} / \mathrm{yr}$ to the west and $13 \mathrm{~mm} / \mathrm{yr}$ to the east for the past $10 \mathrm{Myr}$ [Klitgord and Schouten, 1986; Tucholke and Schouten, 1988]. Several studies have previously been conducted along the western Kane transform and adjacent rift valley. They include dredging and bottom camera tows [Bryan et al., 1981; Karson and Dick, 1983], submersible dives [Zonenshain et al., 1989; Bryan and Fujimoto, 1994], and swath bathymetry with sea surface magnetics [Purdy et al., 1990; Sempéré et al., 1990, 1993; Fujimoto et al., 1994]. These studies, as well as sonar data from the TOBI survey [Allerton et al., 1995], confirm the existence of two spreading segments that exhibit different axial morphologies, both typical of the slow spreading MAR.

Segment 1 extends from the Kane transform to $24^{\circ} 22^{\prime} \mathrm{N}$ ( $\left.\sim 57 \mathrm{~km}\right)$, and segment 2 is located between $24^{\circ} 22^{\prime} \mathrm{N}$ and $24^{\circ} 39^{\prime} \mathrm{N}$ (Figure 3-1). Segment 1 is associated with a narrow, deep, hourglass-shaped valley with steep walls on either side (Figure 3-1). A neovolcanic zone is well-defined along its entire length, except near the bathymetric saddle point, where the rift valley is narrowest. South of $24^{\circ} 16^{\prime} \mathrm{N}$, fault patterns within the valley walls are subparallel to the axis and regularly spaced. To the north, a single fault on the eastern flank becomes increasingly dominant [Allerton et al., 1995].

The boundary between the two segments lies at $24^{\circ} 22^{\prime} \mathrm{N}$, where the NVZ becomes particularly ill-defined and is offset by about $1 \mathrm{~km}$ to the east. This feature has been interpreted as a ridge axis discontinuity by Sempéré et al. [1993]. Coincident with this discontinuity, is an oblique fault cutting across the eastern valley wall with a 
strike of $\mathrm{N} 50^{\circ} \mathrm{E}$. It has been interpreted by Allerton et al. [1995] as an area where differential extension between the relatively $\mathrm{N}-\mathrm{S}$ trending segment to the south and a zone of oblique extension to the north is being accommodated.

Segment 2 exhibits a robust constructional ridge that reaches a height of over 500 $\mathrm{m}$ above the valley floor before terminating abruptly against the obliquely trending western valley wall (Figure 3-1). The valley walls are highly asymmetric in the southern portion of this segment. Extension along the eastern wall is largely accommodated by a single fault that reaches vertical displacements greater than $1 \mathrm{~km}$, whereas the western wall is made up of several small, regularly spaced faults. To the north, before the axial valley jumps $20 \mathrm{~km}$ to the east, the eastern marginal basin broadens and becomes part of an oblique zone trending $45^{\circ}$ to the average spreading direction of the MAR.

The sea surface magnetic field (Figure 3-2) reveals significant intrasegment variation. Anomalies are skewed by an average of $60^{\circ}$ in this region because of geographic location, orientation of the ridge, and the Earth's field direction (inclination is $42^{\circ}$, declination is $\mathrm{N} 18^{\circ} \mathrm{W}$ ). Apart from the low associated with the ridge discontinuity, the CAMH along both segments is weaker and narrower at their centers.

\subsection{Data Collection and Analysis}

The near-bottom magnetic field was measured by a three-axis fluxgate magnetometer attached to the TOBI package (or "fish"). Measurements were averaged into 1-min bins to yield a spatial sampling rate of $60 \mathrm{~m}$ for a nominal towing speed of 2 knots $(\sim 1 \mathrm{~m} / \mathrm{s})$. No transponder was attached to the towed sled, but Global Positioning System (GPS) ship navigation was recorded at 1-min intervals, and cable length was documented every half hour. Instrument position was determined from available information with the help of a cable modeling program [Triantafyllou and Hover, 1990] that includes both instrument and cable hydrodynamics. As a final check, the 


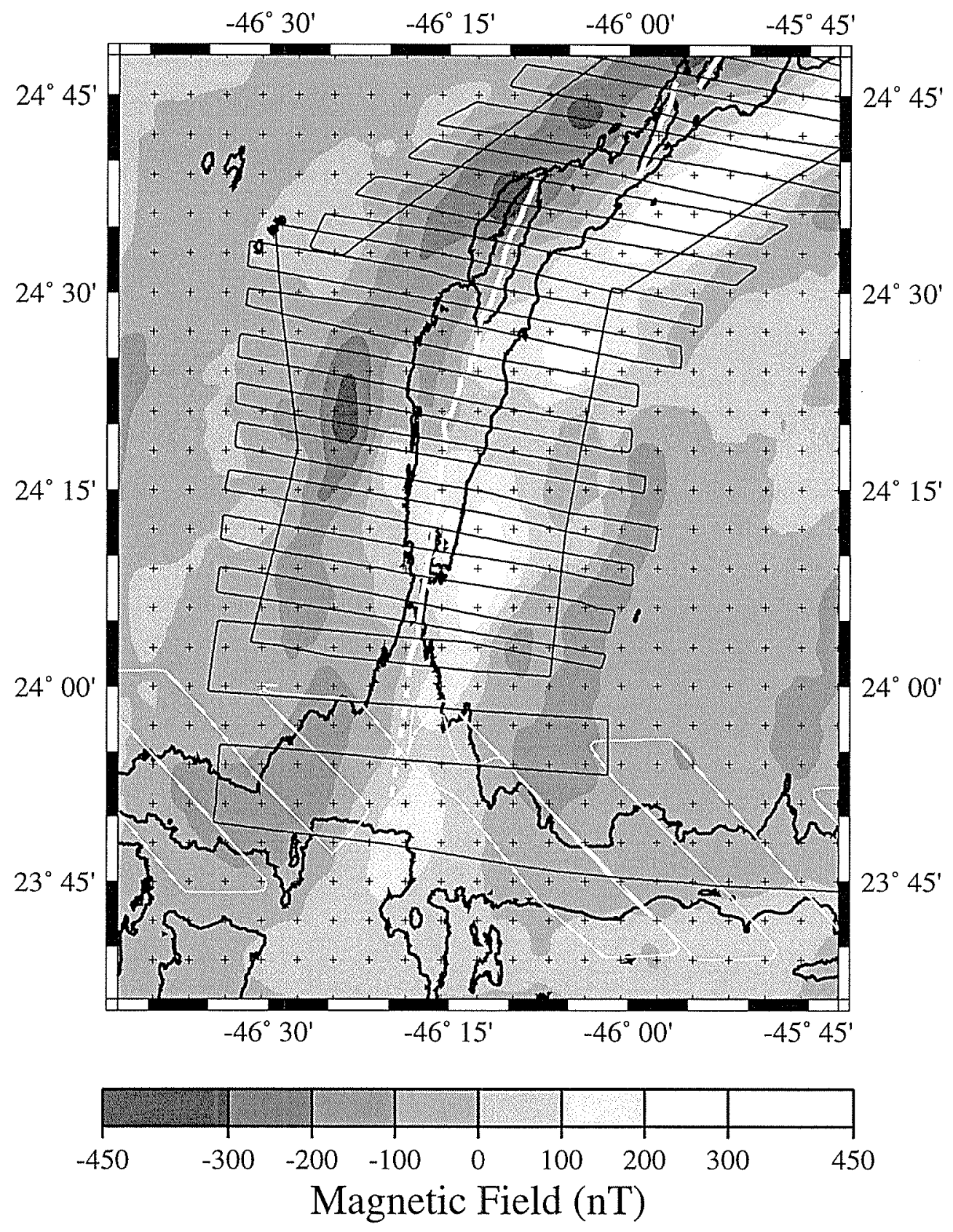

Figure 3-2: The Canadian database [J. Verhoef, personal communication, 1995], gridded at a $0.05^{\circ}$ spacing (crosses), is merged with data obtained during cruise C2908 (white line) and C2909 (black line) [Purdy et al., 1990] aboard the R/V Robert Conrad in 1988 to generate a map of the total sea surface field anomaly. A bold black line marks the $4000-\mathrm{m}$ bathymetric contour, and a bold white line marks the morphologic axis. 
bathymetry measured by the fish was compared to the Sea Beam bathymetry along the predicted track.

Data analysis includes the correction of measurements for instrument attitude, upward continuation of the magnetic field onto a level plane, and inversion of this field for crustal magnetization. Inversion results were compared with forward models generated by known magnetization distributions.

The magnetometer, which is located within the frame of the towed instrument, is affected by the interference between magnetic fields generated by the vehicle and those of geologic origin. By far, the largest source of interference is the instrument's permanent magnetization [Mies, 1986]. This remanent magnetization either constructively or destructively adds to the true field, depending on the angle between them, and must be corrected. Removal of the permanent field interference effect was best achieved by using a cosine fit method, whereby field strength is individually plotted as a function of the three axes of rotation, and best fitting cosine curves are removed from each. Although the true interference effect is not perfectly sinusoidal, this becomes a good approximation when the Earth's field is much larger than the instrument's permanent field [Mies, 1986]. This method corrects only total field values. Hence individual fluxgate components are not presented in this paper. As a final correction, the International Geomagnetic Reference Field (IGRF) was determined at each measurement location [International Association of Geomagnetism and Aeromagnetism (IAGA) Working Group I, 1987] and subtracted from the data, leaving the total field anomaly (Figure 3-2).

Since current inversion methods require measurements to be on a level plane, the data acquired in this draped survey were upward continued onto such a plane by the standard Schwarz-Christoffel transformation [Parker and Klitgord, 1972]. In this study, we also use two new reduction methods: the Guspi [1987] method and the approximate equivalence method of Pilkington and Urquhart [1990]. The Guspi method represents a summation of iterative corrections to the measured field in the frequency 
Table 3.1: Corner Wavelengths of Filters

\begin{tabular}{cccc}
\hline & Reduction Filter & $\begin{array}{c}\text { Inversion Filter } \\
\text { Wavelength, km }\end{array}$ & $\begin{array}{c}\text { Profile } \\
\text { Location }\end{array}$ \\
\hline 1 & Wavelength, km & 0.6 & 1.1 \\
2 & Segment $2\left(24^{\circ} 34^{\prime} \mathrm{N}\right)$ & 1.2 & 1.2 \\
3 & Nouth End Segment $1\left(24^{\circ} 21^{\prime} \mathrm{N}\right)$ & 1.0 & 1.6 \\
4 & Inside Corner $\left(23^{\circ} 54^{\prime} \mathrm{N}\right)$ & 0.8 & 1.2 \\
\hline
\end{tabular}

domain and is based on the substitution of the equation for the theoretical field on horizontal plane due to a general source distribution into the equation for the field on an uneven surface [Guspi, 1987]. This method tends to amplify high frequencies and therefore requires low-pass filtering within each iteration. The method of Pilkington and Urquhart [1990] is based on the approximate equivalence that exists between the field measured on an uneven observation surface due to a source distribution on a level plane and the field on a level plane due to an equivalent distribution on a mirror image of the original observation surface [Pilkington and Urquhart, 1990]. The reduction process solves for an equivalent source distribution on this mirror image surface and then calculates a reduced field from these sources. The process of calculating the equivalent source distribution is essentially a downward continuation and therefore must be filtered. In all upward continuation methods, the passband of each filter is dependent on the frequency content of the data and the continuation distance of each profile (see Table 3.1 for filter values).

Upward continued data were inverted for crustal magnetization using the iterative Fourier method developed by Parker and Huestis [1974]. The direct inversion method of Hussenoeder et al. [1995] was also examined, in which the approximate equivalence concept of Pilkington and Urquhart [1990] is used to solve for crustal magnetization directly from the uneven observation track. All inversions assume a 500-m-thick source layer and a geocentric dipole direction for the magnetization. 


\subsection{Results}

Figure 3-3 shows our deep tow magnetization profiles positioned within the magnetization map derived from sea surface measurements. In order to obtain a reliable spreading rate estimate, the reversal pattern from both sea surface and deep tow magnetization solutions was determined as a function of distance from the neovolcanic axis. Reversal identifications were extended out to the Matuyama/Gauss reversal at 2.6 Ma [Cande and Kent, 1995], over which the interval spreading rate was found to be fairly constant. The magnetic anomaly pattern suggests a half spreading rate to the west of $13.1 \mathrm{~mm} / \mathrm{yr}$, whereas to the east, it increases from less than $10 \mathrm{~mm} / \mathrm{yr}$ near the fracture zone to $13 \mathrm{~mm} / \mathrm{yr}$ at segment 2, resulting in more closely spaced reversals at the inside corner high (Figure 3-3). This pattern, however, places the pole of opening for the Atlantic in an unrealistic position, namely, a few hundred kilometers to the south, and is more likely an indicator of temporal variability in spreading rate. Calculations extending past anomaly 5 show the average spreading rate in this area to be constant for the past $10 \mathrm{Myr}$ [Tucholke and Schouten, 1988]. Although earlier bathymetric data show little evidence for propagating rifts [Sempéré et al., 1993], high-resolution data, collected aboard the Japanese support vessel Yokosuka in 1994, resolve the off-axis scars of several southward propagating rifts [Fujimoto et al., 1994; N. Seama, personal communication, 1995]. Two have since terminated at the transform, and a third traces the growth of segment 2 at the expense of segment 1. Since outer pseudofaults mark a discontinuous jump to older crust on the side farther from the rift axis, the presence of propagating rifts may explain the apparent variation in spreading rate along axis.

Short-wavelength variations seen in the deep tow profiles are not present in the sea surface data. This is dramatically shown in Figure 3-4, where magnetizations derived from sea surface measurements along the predicted deep tow tracks are plotted against magnetizations derived from the near-bottom data. While the Brunhes/Matuyama reversal is consistently detected in the sea surface data, the Jaramillo subchron is not. 


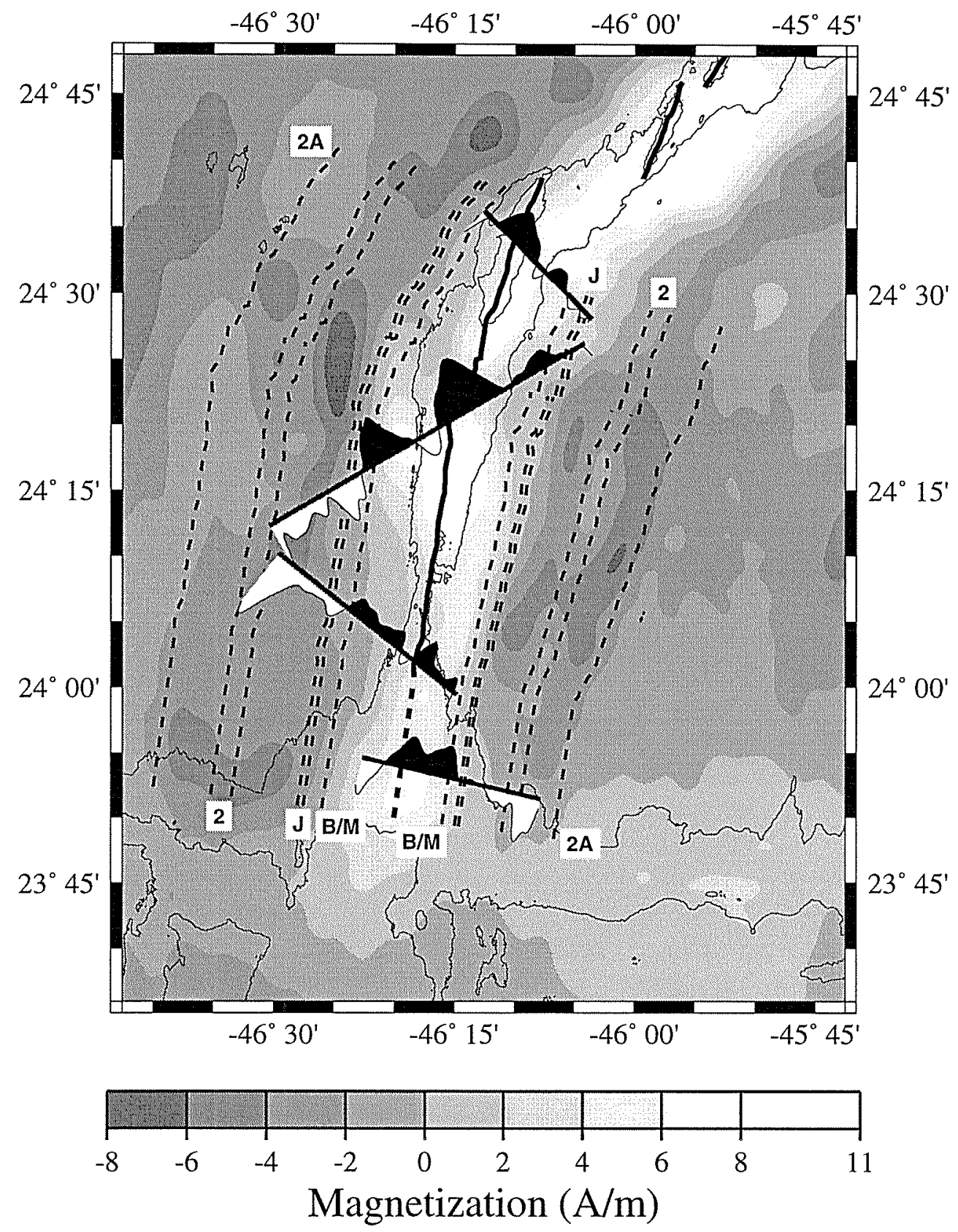

Figure 3-3: Magnetization map derived from sea surface data for the study area. Deep tow magnetizations, calculated from the Guspi upward continued field, are plotted as solid wiggles along straight-line approximations of the vehicle track. A thick line represents the morphologic axis, and dashed lines are reversal identifications based on sea surface and deep tow data assuming a constant spreading rate. The 4000-m bathymetric contour (thin line) is also plotted. The Brunhes-Matuyama (B/M) reversal, Jaramillo (J) subchron, and anomaly 2 and $2 \mathrm{~A}$ are labeled. 


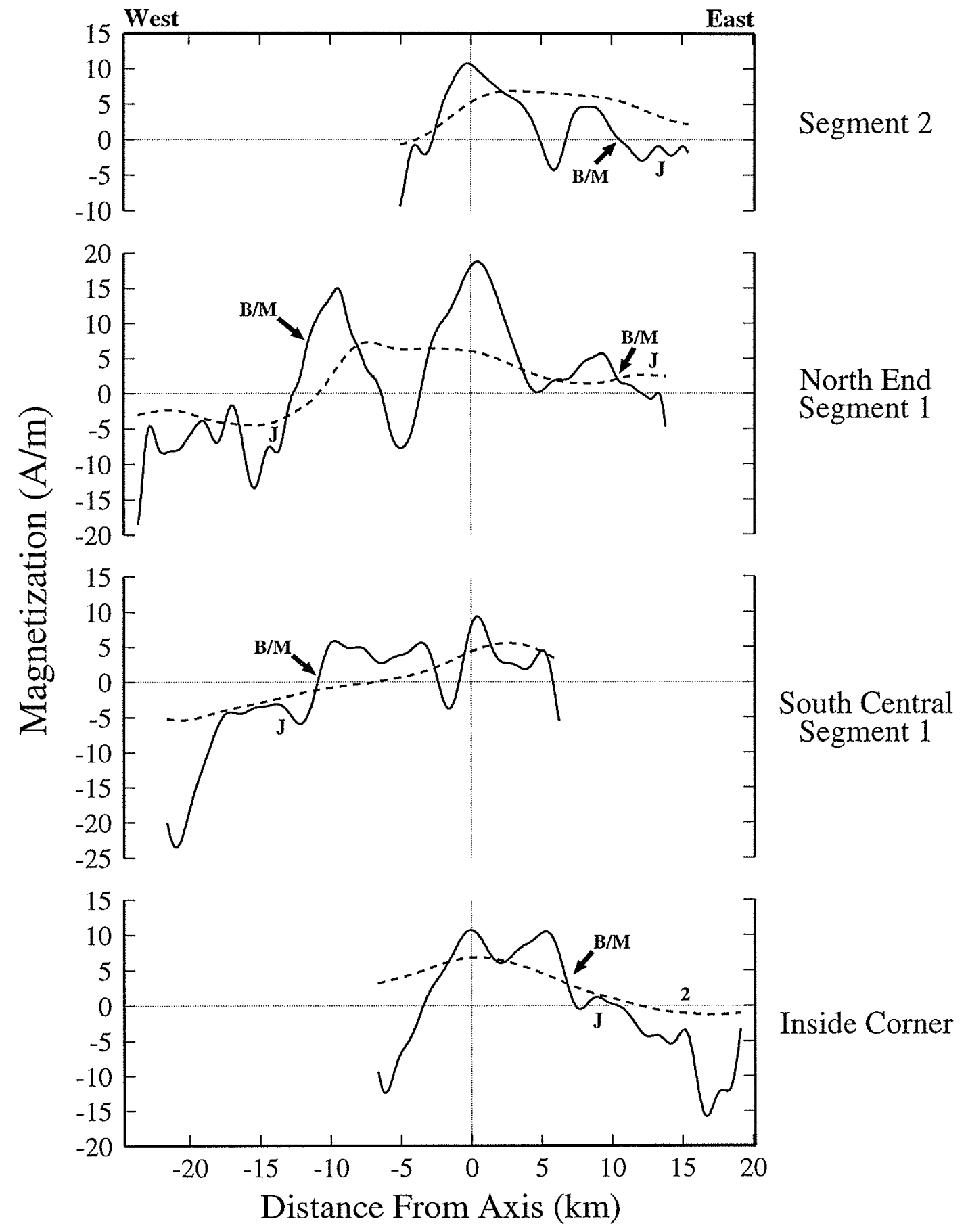

Figure 3-4: Comparison of calculated sea surface (dashed) and deep tow (solid) magnetization profiles along the four TOBI track lines versus distance from the axis. The Brunhes-Matuyama reversal, Jaramillo subchron, and anomaly 2 are labeled. 
There are several explanations for the frequent disagreement in the exact location of reversal boundaries between the sea surface and near-bottom profiles. First, since potential fields from a dipole source falloff as $1 / r^{3}$, near-bottom data are more sensitive to variations in shallow crustal structure than are sea surface measurements. Any difference between shallow magnetic structure and depth-integrated structure will manifest itself as a difference between the two magnetization profiles. Second, the sea surface magnetization map is a highly filtered version of the true crustal magnetization. The near-bottom profiles show that the Brunhes/Matuyama reversal is a well-defined and rapid transition, one which filtering only serves to distort. Third, the inversion method of Parker and Huestis [1974] is Fourier-based and is therefore insensitive to DC shifts and susceptible to spectral leakage near the ends of profiles that contain linear trends in the field. This poses no problem if the spatial extent of the magnetic profile is large enough that neither a linear trend nor a nonzero mean exists. However, for data sets of limited extent, or for those dominated by a single polarity, the common practice of subtracting out the mean or trend of the field prior to inverting may result in a significant loss of information. We believe that the linear trends and nonzero means in these deep tow profiles are geologically significant (see Figure 3-4). We have therefore retained them in our magnetization solutions by applying, prior to inversion, sufficiently long buffers that exponentially decay to the mean of each profile. This approach enables us to suppress a majority of the spectral leakage.

Along-axis amplitude variations in the CAMH are evident in both the sea surface and deep tow data. The CAMH weakens toward the center of both segments, forming a saddle shape. Figure 3-3 shows that the CAMH is significantly stronger near the ridge-transform intersection (RTI) and on either side of the junction between the two segments $\left(24^{\circ} 18^{\prime} \mathrm{N}\right.$ and $\left.24^{\circ} 27^{\prime} \mathrm{N}\right)$ than at each segment's center. In fact, the magnetization strength at the north end of segment 1 is double that at its center (Figure 3-4). The magnetization along each of the four deep tow profiles is shown 
with the bathymetry measured along track in Figures 3-5a through 3-5d. We will now discuss each profile in turn.

\subsubsection{Profile 1: Segment $2\left(24^{\circ} 34^{\prime} \mathrm{N}\right)$}

The northern profile crosses the center of segment 2 and extends from the western flank of the median valley across the broad median valley floor into the rift mountains (Figure 3-5a). It includes the eastern Brunhes/Matuyama reversal and Jaramillo subchron. The two overlapping constructional features on the valley floor, shown in bathymetric cross section, have been identified in sonar images as flat-topped volcanos cresting a robust neovolcanic ridge. They mark the location of most recent volcanic activity (i.e., the neovolcanic zone). A pronounced CAMH reaches its maximum amplitude of $11 \mathrm{~A} / \mathrm{m}$ over the western ridge, indicating that the western side is the current location of active emplacement. Photoanalysis of WASP camera footage confirms this observation by showing the eastern flank to be considerably older than the fresh flows extending into the western marginal basin [N. K. Lawson, personal communication, 1995]. The CAMH is flanked on both sides by a sharp drop in magnetization into what is apparently a narrow zone of reversely magnetized crust (Figure 3-5a). The eastern magnetic "reversal," or low, corresponds to the steepest slope in the valley wall, which is made up of a single normal fault with a vertical displacement of over $500 \mathrm{~m}$. More positively magnetized crust outside of this scarp corresponds to what appears to be a back-tilted crustal block. There is indication of a similar pattern in the west, but because of its proximity to the end of the profile, the signal is likely to be partially corrupted by edge effects.

\subsubsection{Profile 2: North End of Segment $1\left(24^{\circ} 21^{\prime} \mathrm{N}\right)$}

The longest of the four profiles traverses the rift valley near the north end of segment 1 (Figure 3-5b). It extends into the rift mountains on both sides and includes the Brunhes/Matuyama reversal, Jaramillo subchron, and anomaly 2 in the west. Two 


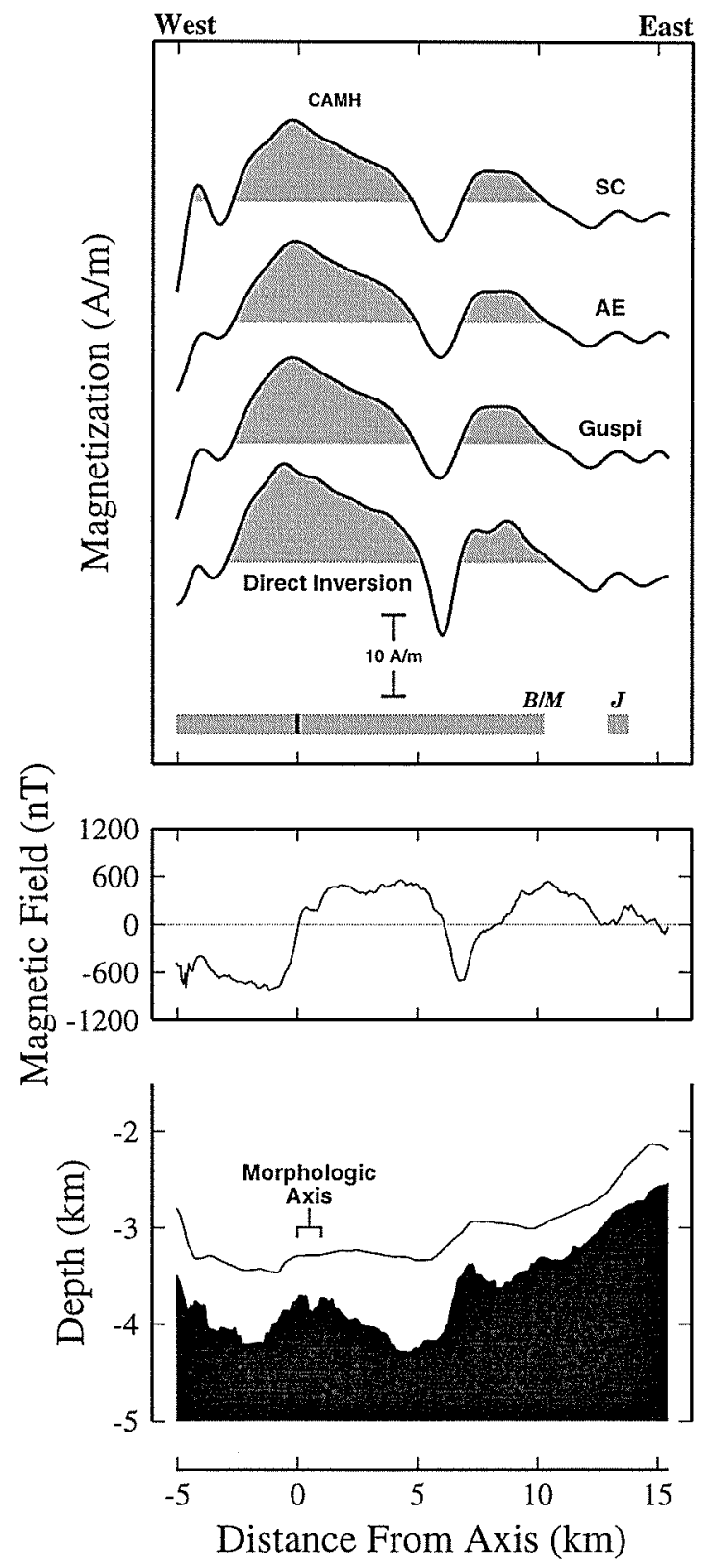

Figure 3-5a: Deep tow magnetic profile crossing segment 2 at $24^{\circ} 34^{\prime} \mathrm{N}$. (bottom) Bathymetry and instrument track versus distance from ridge axis. The morphologic axis [D. K. Smith, personal communication, 1995] is marked in all but the southernmost profile, where it is less defined. (middle) Measured field anomaly. (top) Magnetization solutions using four methods: Schwarz-Christoffel (SC) [Parker and Klitgord, 1972], approximate equivalence (AE) [Pilkington and Urquhart, 1990], [Guspi, 1987], and direct inversion [Hussenoeder et al., 1995]. Shaded bars indicate normal polarity intervals, based on the reversal picks of Figure 3-3. Reversals and normal polarity chrons are labeled. Upward continuations and inversions were taken to 20 iterations. The inversion assumes a $500 \mathrm{~m}$ thick source layer and a geocentric dipole direction for the magnetization. 


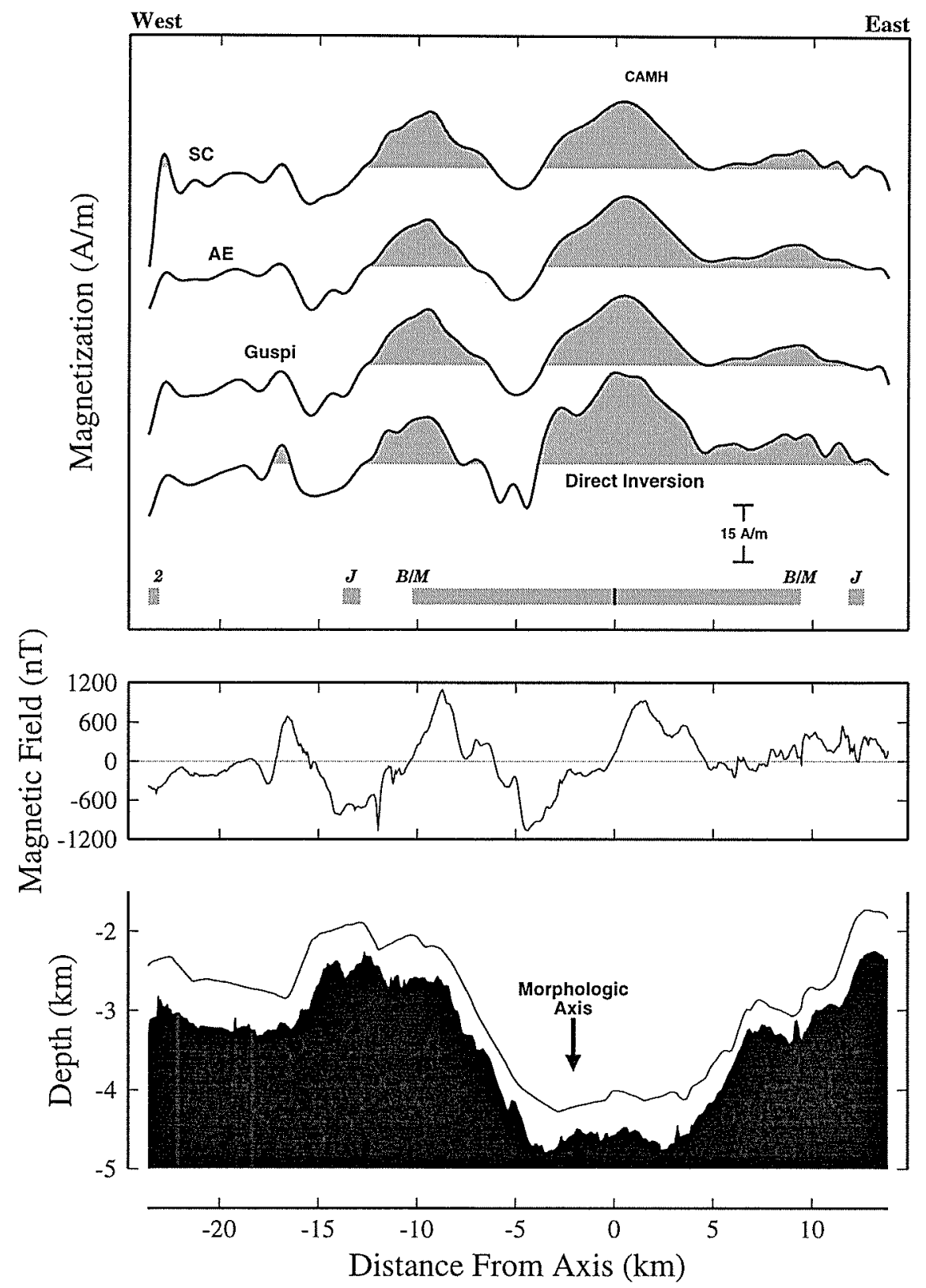

Figure 3-5b: Same as Figure 3-5a, except for north end of segment 1 at $24^{\circ} 21^{\prime} \mathrm{N}$.

separate ridges occupy the valley floor. The eastern ridge is continuous with the NVZ of segment 2. Previous results from a submersible dive to the north of this area show that this neovolcanic ridge has young lavas only at the crest and that its flanks are heavily faulted and fractured [Zonenshain et al., 1989]. The western ridge corresponds to the NVZ of segment 1. TOBI side-scan sonar shows it to be less 
tectonically disrupted and more hummocky in nature than its bathymetrically higher counterpart to the east. Yet the CAMH reaches its maximum of $\sim 20 \mathrm{~A} / \mathrm{m}$ (Figure 3$5 b)$ over the eastern bathymetric high. A sharp reduction in amplitude coincides with the initial scarp of the eastern valley wall and seems to predict negatively magnetized crust within the western wall in a pattern similar to the previous profile. Allerton et al. [1995] report that the large normal fault at the eastern wall exhibits over 1 $\mathrm{km}$ of vertical displacement and that midcrustal rocks were dredged near its base. TOBI side-scan sonar images show that mass wasting is extensive over large areas of this fault, whereas the western wall is made up of several smaller scarps that localize extension into discrete zones, resulting in less wholesale disruption of the crust.

\subsubsection{Profile 3: South Central Part of Segment $1\left(24^{\circ} 02^{\circ} \mathrm{N}\right)$}

A much weaker $(\sim 9 \mathrm{~A} / \mathrm{m})$, narrower $\mathrm{CAMH}$ is associated with the neovolcanic ridge just south of the Narrowgate section of segment 1 (Figure 3-5c). The CAMH peak is associated with a small, hummocky ridge in the center of the valley floor. Photoanalysis of the WASP tow across the bathymetric saddle of this segment shows a poorly developed NVZ with a sharp contrast in age at its flanks, based on sediment cover and degree of tectonism [N. K. Lawson, personal communication, 1995]. The proximity of heavily tectonized crust correlates well with the inversion solutions, which show a sharp drop in amplitude very close to the ridge. Although the nadir of the TOBI profile is nearly $10 \mathrm{~km}$ to the south of the camera tow, and the valley floor is slightly wider at this location, Allerton et al. [1995] places both locations within the same structural domain. The valley walls are highly symmetric, with normal faults spaced every $2 \mathrm{~km}$. A similar spatial frequency is also seen in the slightly oscillatory magnetization pattern of the valley walls (Figure 3-5c). 


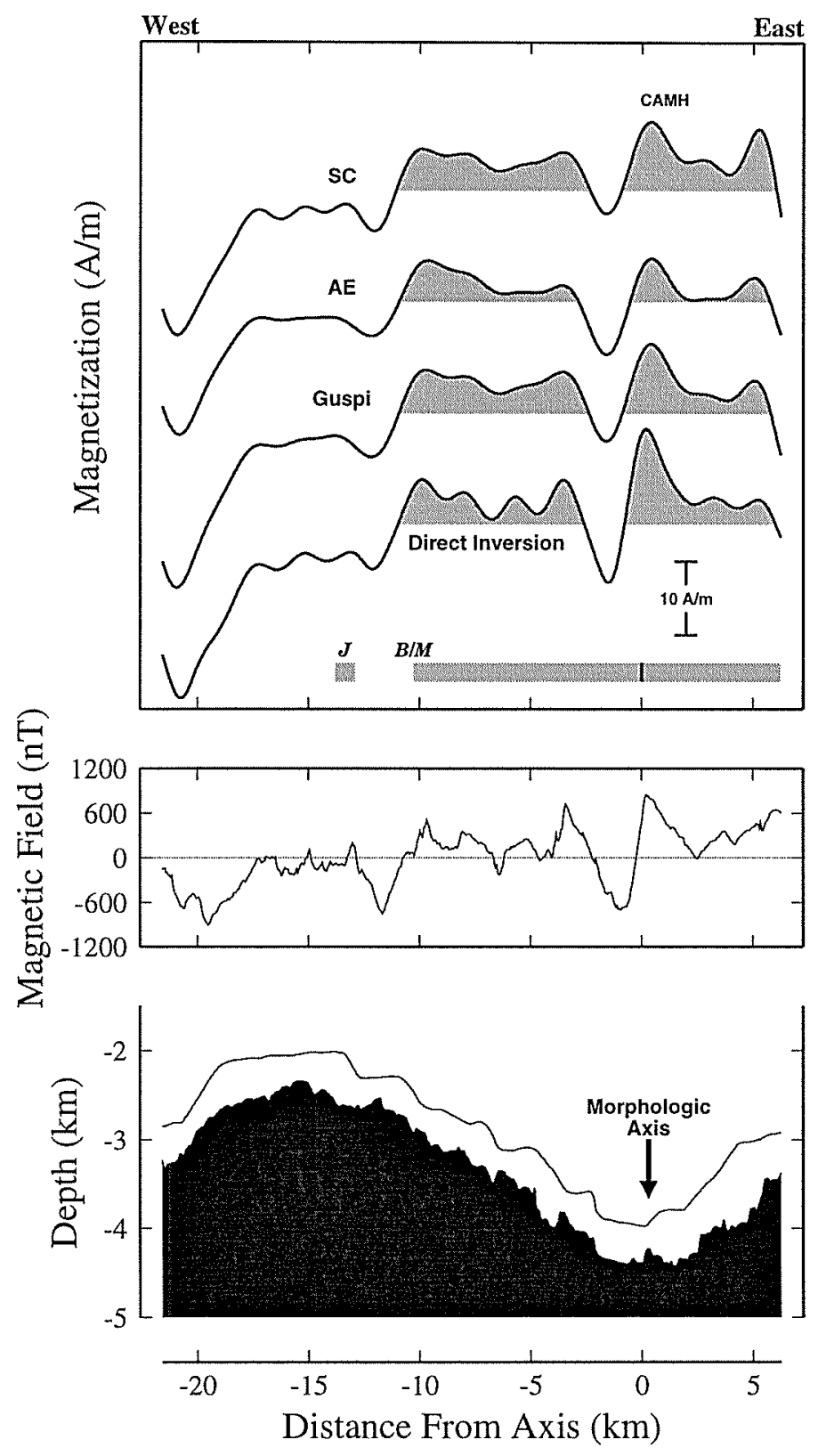

Figure 3-5c: Same as Figure 3-5a, except for south central part of segment 1 at $24^{\circ} 02^{\prime} \mathrm{N}$. 


\subsubsection{Profile 4: The Nodal Deep and Inside Corner High $\left(23^{\circ} 54^{\prime} \mathrm{N}\right)$}

The CAMH gains strength toward the RTI, and reaches an amplitude of about 11 $\mathrm{A} / \mathrm{m}$ at the southernmost TOBI profile. A double-peaked magnetization high occupies much of this profile (Figure 3-5d). Two submersible dives conducted by Zonenshain et al. [1989] in this area found what they have interpreted to be a relict neovolcanic ridge $5 \mathrm{~km}$ to the east of the active NVZ. This relict ridge coincides with the eastern magnetization peak predicted by all four methods (Figure 3-5d). The sharp drop in amplitude on the eastern flank of the relict ridge is interpreted to be the Brunhes/Matuyama reversal, although its location at base of the valley wall suggests that disruption of the magnetic source layer may also be important. Submersible dives have mapped upper gabbro exposures as well as extensive talus fans at the wall of the inside corner high [Zonenshain et al., 1989], even though previous dredging and deep tow studies report a scarcity of plutonic rocks [Karson and Dick, 1983; Bryan and Fujimoto, 1994]. This would indicate a high degree of vertical uplift and tectonism.

\subsection{Discussion}

We consistently observe two features in the deep tow profiles: a narrow CAMH and valley wall magnetization lows. Characterization of these anomalies, including their location and variability, is important to our understanding of the volcano-tectonic processes occurring in slow spreading environments. We now discuss implications associated with the fine-scale magnetic structure recorded in these near-bottom magnetic profiles.

In order to better interpret the deep tow results, forward models of each profile were constructed (Figures 3-6a through 3-6d). Given an assumed magnetization distribution, the field was calculated along-track in the actual profile geometry using 


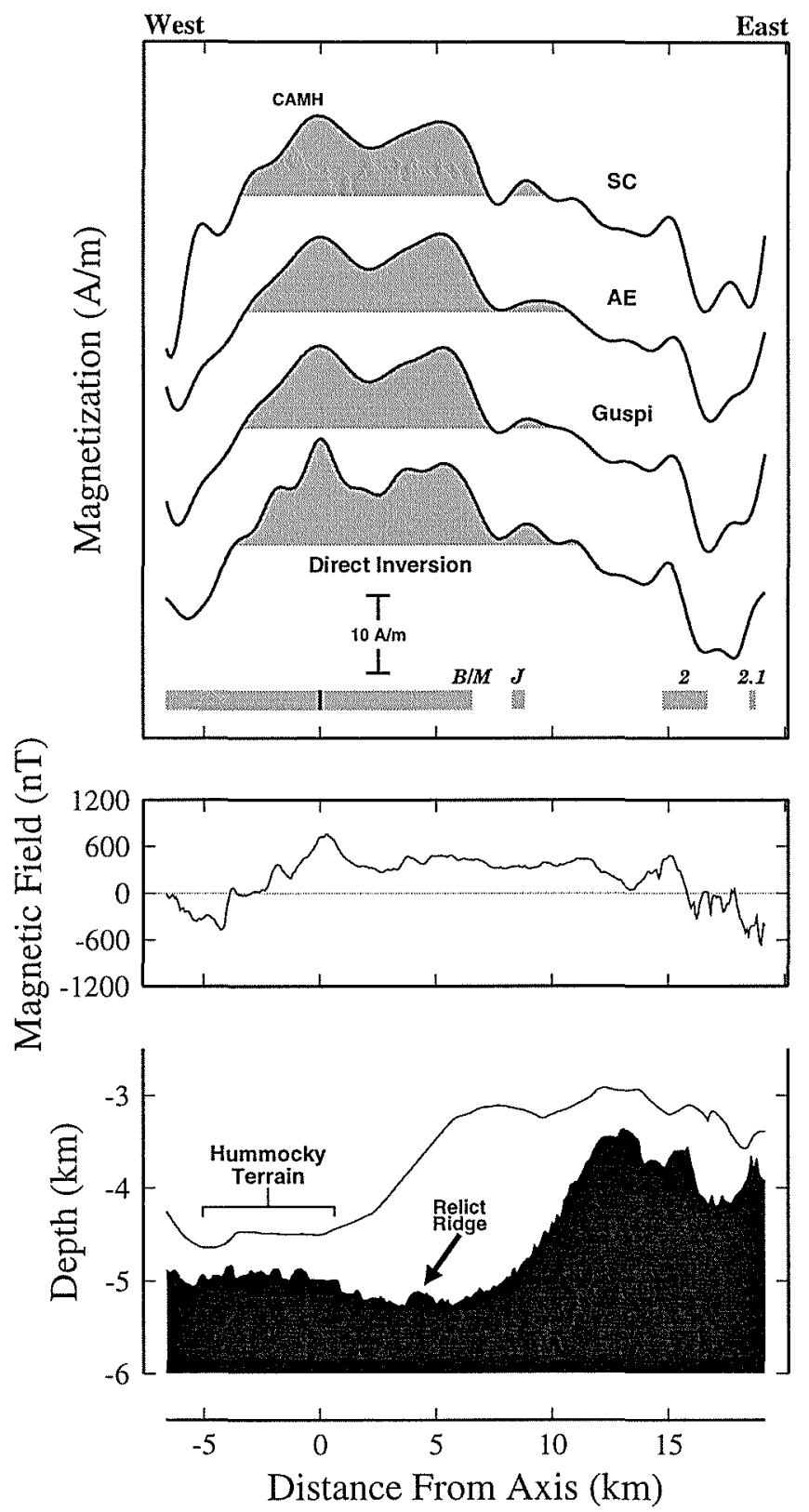

Figure 3-5d: Same as Figure 3-5a, except for nodal deep and inside corner high at $23^{\circ} 54^{\prime} \mathrm{N}$.

an implementation of Talwani and Heirtzler's [1964] analytic method [Won and Bevis, 1987]. The calculations assume two dimensionality and a 500-m-thick source layer. The input magnetic reversal pattern is based on recently published chronologies [Cande and Kent, 1995] (see Figure 3-6a for spreading rates). Two features have been included in the input magnetization: a time-dependent exponential amplitude decay, 
and discrete zones of zero magnetization at the axial valley walls. An exponential decay constant of $150 \mathrm{kyr}$ (i.e., decay to $1 / e$ the maximum value in $2 \mathrm{~km}$ for a 13 $\mathrm{mm} / \mathrm{yr}$ spreading rate) was chosen because of its overall best fit to the decay rate of these profiles. Although the peak amplitude of the input magnetization was varied between profiles in order to best fit the magnetic data, all forward models assumed the same decay rate to a background amplitude of $4 \mathrm{~A} / \mathrm{m}$. Nonmagnetic regions were varied in width and location to create a best fitting model. They were included to test the hypothesis that the valley walls are areas of magnetic source destruction.

Several specific questions concerning the accretion and subsequent alteration of ocean crust can be addressed by examining the deep tow magnetic anomalies in relation to the forward models and geologic observations [e.g., Zonenshain et al., 1989; Allerton et al., 1995]. The accretionary process is examined chiefly by studying the origin of the CAMH and its along-axis variability, whereas tectonic processes are examined by studying the valley wall magnetic lows.

\subsubsection{The Central Anomaly Magnetic High}

Does the CAMH accurately locate the most recent extrusive activity? The CAMH in all four profiles is characterized by a narrow peak $(<2 \mathrm{~km}$ half width to half magnetization on average) that corresponds to the axial volcanic ridge. If volcanic events were spatially distributed within a wide zone of accretion, a much broader magnetization high would result [Schouten and Denham, 1979]. The sharp peak of the CAMH not only suggests a precise location for focused accretion, but it also implies that the accretionary process is not responsible for Atlantic magnetic anomaly variability. The fact that the location of the CAMH consistently agrees with observations from both side-scan sonar data and camera tows is further evidence that this feature is a powerful complement to imaging techniques in locating the neovolcanic zone.

Disagreement does exist between TOBI side-scan images and near-bottom magnetic measurements in profile 2 , where the western volcanic ridge is more hummocky 

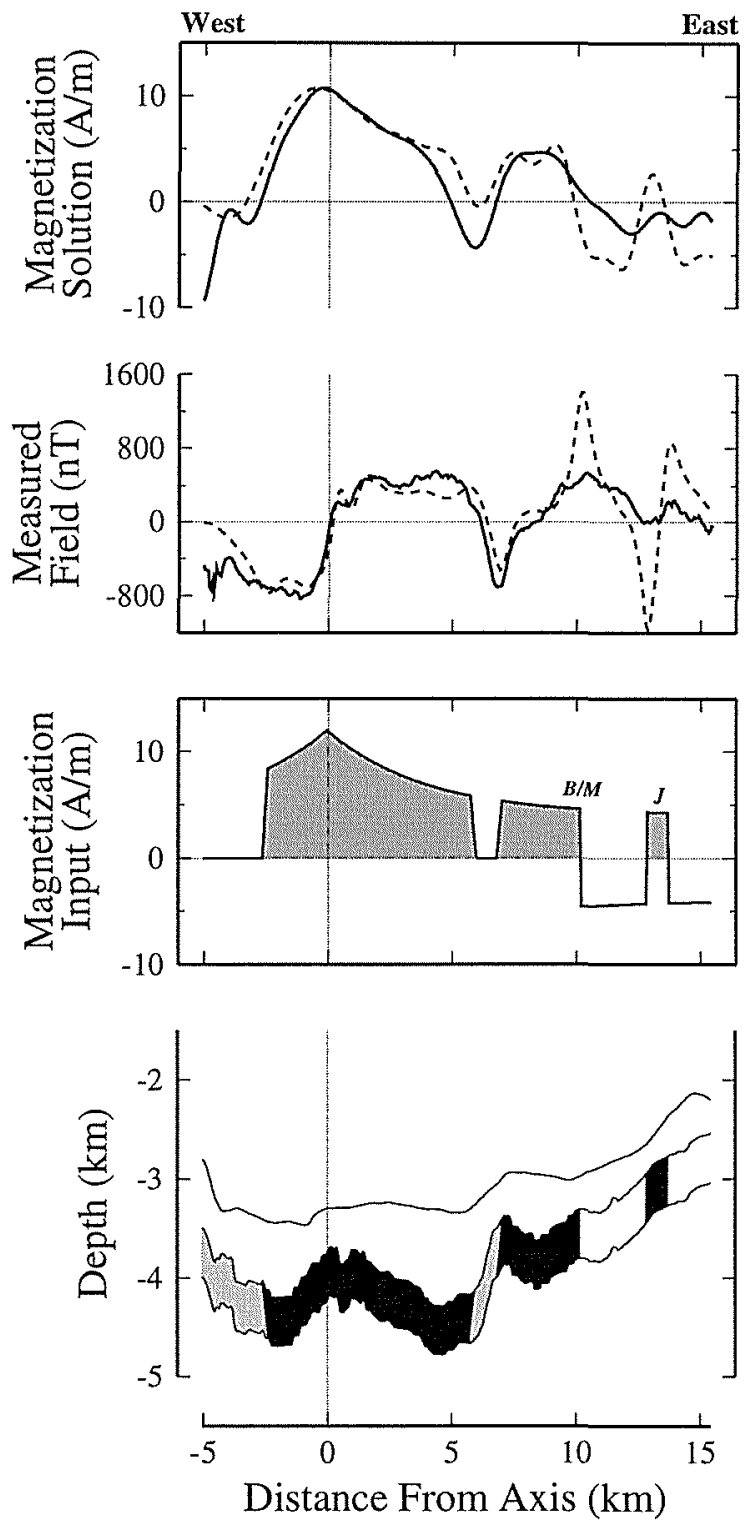

Figure 3-6a: Forward model of the profile crossing segment 2. (bottom) Bathymetry and instrument track as in Figure 3-5a. Modeled areas of magnetic source destruction (gray shaded zones) are shown within the positively (black) and negatively (white) magnetized crust, assumed to be $500 \mathrm{~m}$ thick for all inversions. (middle) The model magnetization distributions, which include an exponential magnetization decay rate (150 kyr time constant) and zones of source destruction flanking the CAMH. Reversals and normal polarity events are labeled. All models use a spreading rate of $13 \mathrm{~mm} / \mathrm{yr}$ to the west. Decreasing spreading rates of $13.1,12,10$, and $8.4 \mathrm{~mm} / \mathrm{yr}$ to the east are used to model the profiles in Figures $3-6 \mathrm{a}, 3-6 \mathrm{~b}, 3-6 \mathrm{c}$, and 3-6d respectively, based on reversal identifications [Cande and Kent, 1995]. (top) The model field anomaly and magnetization (dashed) plotted against values derived from the data (solid). The magnetization solutions are those obtained by the Guspi upward continuation method followed by inversion. 

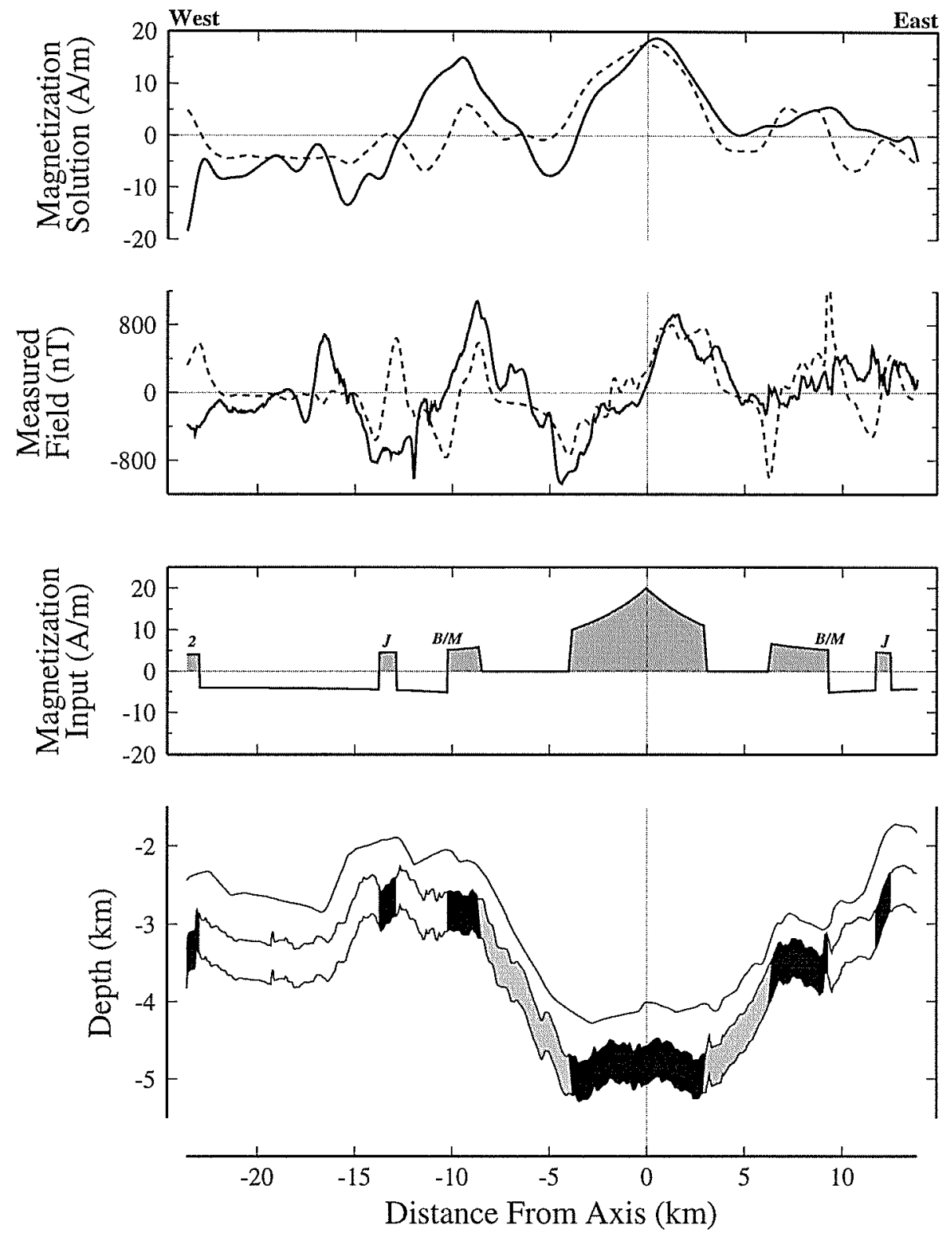

Figure 3-6b: Same as Figure 3-6a, except for north end of segment 1.

and less tectonized, while the eastern ridge exhibits a higher magnetization. This enigma may only be resolved by the analysis of samples gathered from this location. One must keep in mind that the magnetic signal is a depth-integrated function representing the time-averaged accretion of new material. If, on average, the eastern ridge is younger, but the western ridge is covered by a thin veneer of fresh lava, the conclu- 

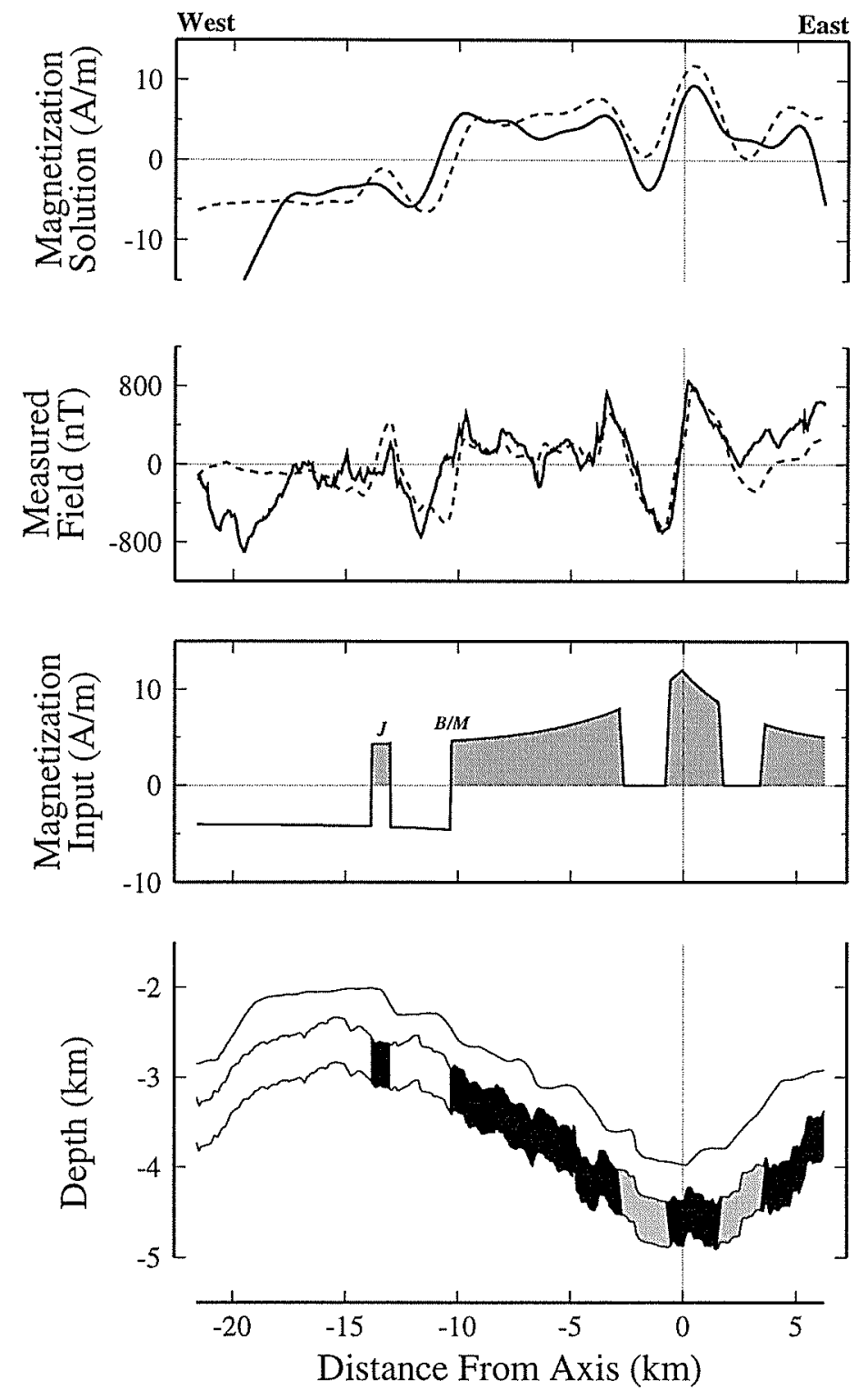

Figure 3-6c: Same as Figure 3-6a, except for south central part of segment 1.

sions obtained from these two observations would conflict as they do now. Overall, however, the CAMH is an excellent indicator for the location of most recent volcanic activity. The relict ridge in profile 4 may be the result rift propagation, recorded in the off-axis bathymetry [Fujimoto et al., 1994; N. Seama, personal communication, 1995]. Rift propagation occurs on timescales much longer than those used to describe the emplacement and evolution of the magnetic source layer. However, the creation 

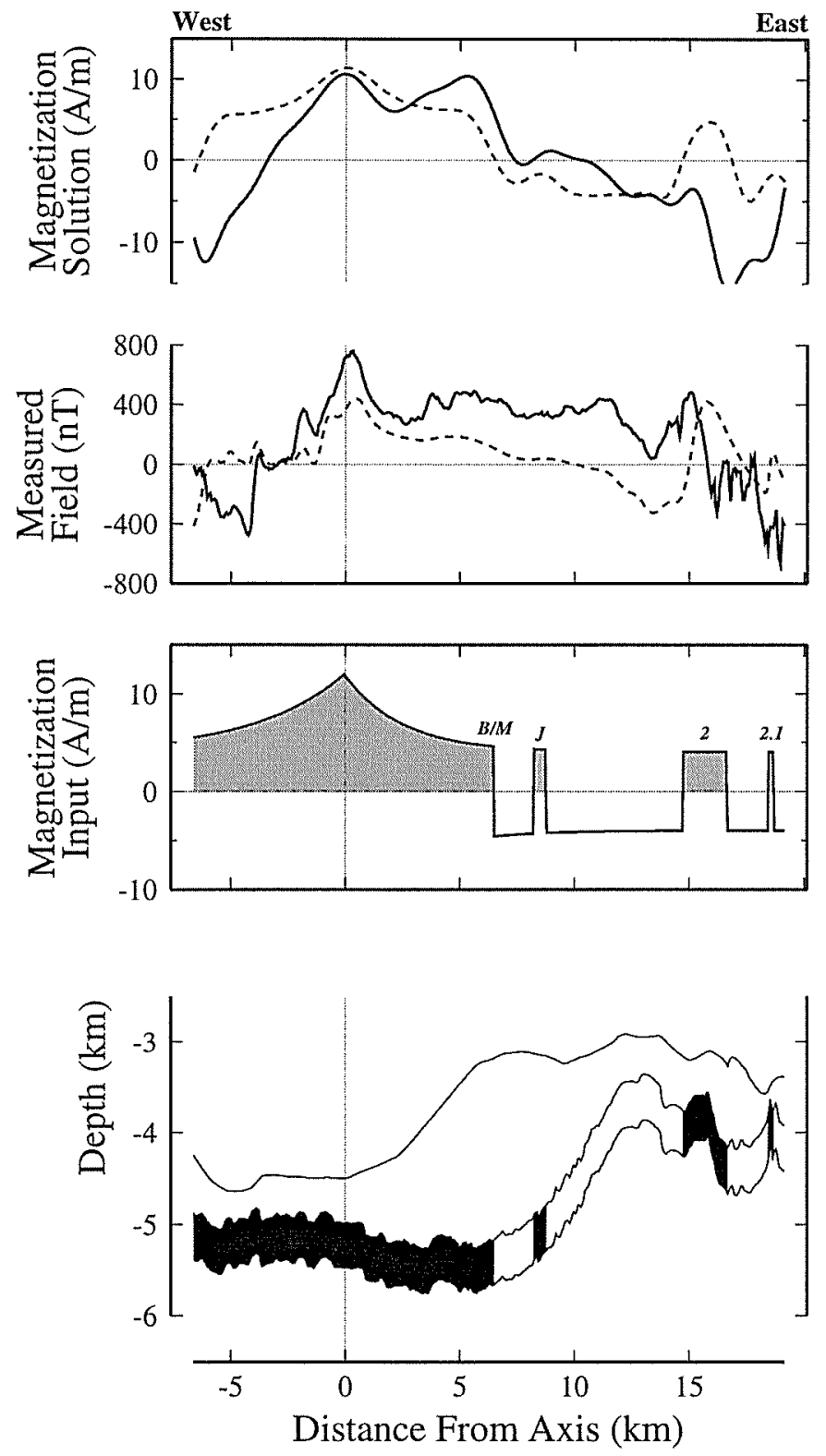

Figure 3-6d: Same as Figure 3-6a, except for nodal deep and inside corner high.

of this relict ridge allows us to determine the style of accretion that has occurred along that part of the spreading segment in the past. The narrow, peaked nature of the magnetic high corresponding to the relict ridge is further indication that volcanic emplacement is focused at slow spreading centers.

What is responsible for the along-axis variability of the CAMH? Both the sea 
surface and deep tow data agree that the axial magnetic high is much stronger and wider at the distal ends of both segments than at their bathymetrically shallow centers. A number of different hypotheses have been proposed to explain this behavior. The Curie isotherm model considers that each ridge segment is located above a hot zone of mantle upwelling [e.g., Whitehead et al., 1984; Lin et al., 1990]. Such a plume would impose a steep thermal gradient in the crust and hence thin the effective magnetic source layer by moving the Curie isotherm to a shallow level. Isotherms would deepen toward the distal ends of the segment, allowing a greater proportion of crust to contribute to the magnetic signal. This model predicts a more pronounced effect in segments that are volcanically active, such as segment 2. Yet, it is segment 1 that most visibly exhibits a saddle-shaped CAMH. This model also predicts that as crust is transported off-axis, the along-isochron magnetization pattern would disappear, since isotherms would become horizontal. However, high-amplitude magnetizations associated with segment ends are frequently observed to continue off-axis (Figure 3-3, west). This suggests that although this model may be physically plausible, it most likely accounts for only a small portion of the along-axis magnetic variation of these two segments.

A second hypothesis is that valley wall proximity, and the alteration associated with them, causes a reduction in the sea surface expression of the CAMH toward segment centers. In a narrowgate geometry, such as segment 1 , the valley walls converge toward the center of a segment, bringing the effects of tectonic disruption closer to the neovolcanic zone and reducing the amount of crust available to generate a magnetic high. These deep tow measurements are able to resolve a narrow CAMH and find that it has a lower magnetization than at the segment ends (Figure 3-6c). If the magnetization of fresh basalts at the segment center were as high as that for basalts at its distal ends, then the deep tow profile would be able to resolve a narrow, high-amplitude CAMH. The fact that it does not implies that the geometry argument is not valid and that the magnetization at segment ends is higher for other reasons. 
This brings us to the hypothesis that magnetization highs at segment ends are the result of increased iron and titanium content within the basalts. In this model, material at the distal ends fractionate over a longer time period in smaller, more isolated magma chambers, thereby increasing the percentage of magnetic minerals in the bulk composition of erupted basalts [Christie and Sinton, 1981]. This model has also been proposed as the source of high-amplitude magnetizations observed along the propagating limb of large-offset overlapping spreading centers [Sempéré and Macdonald, 1986; Sempéré, 1991]. Preliminary analysis of dredged samples along segment 1 has found the most primitive basalts at the Narrowgate $\left(\mathrm{Fe}_{2} \mathrm{O}_{3} \quad 9.5\right.$ wt $\%$ and $\mathrm{TiO}_{2} 1.2 \mathrm{wt} \%$ ), and a wide range of compositions, including high $\mathrm{Fe}$ and Ti basalts $\left(\mathrm{Fe}_{2} \mathrm{O}_{3} 10.75 \mathrm{wt} \%\right.$ and $\left.\mathrm{TiO}_{2} 1.65 \mathrm{wt} \%\right)$ at the segment ends [N. K. Lawson, personal communication, 1995]. Numerous studies have previously related the Fe and Ti content in basalts to their remanent magnetization [e.g., Vogt and Johnson, 1973; Vogt, 1979; Weiland et al., 1995]. Applying our results to the relationship determined by [Weiland et al., 1995] for the South Atlantic, we predict an increase in magnetization from 16 to $32 \mathrm{~A} / \mathrm{m}$ for the changes in Fe and Ti content measured along the axis of segment 1 . This is in agreement with the twofold increase in magnetization shown in the deep tow magnetic profiles (Figure 3-4). The low-amplitude CAMH associated with the robust NVZ of segment 2 (Figure 3-3) may also partially be a result of a basalt chemistry relatively low in $\mathrm{Fe}$ and $\mathrm{Ti}$.

It is not surprising that the rock sample measurements exhibit a higher magnetization than do the deep tow profiles, since the field represents a depth-integrated measure of source strength and dredges most likely sample recently erupted basalts capping the neovolcanic ridge. According to this argument, along-axis compositional variations in erupted material are largely responsible for the increase in magnetization observed toward segment ends. Care must be taken in making a direct comparison between iron content and remanent magnetization, however, because factors other than Fe and Ti content, such as magnetic mineralogy and grain size, can also control 
magnetization intensity [e.g., Watkins, 1974; Sempéré, 1991]. Thus, while we consider rock chemistry to play a key role in the along-axis variability of the CAMH, contributions from various other models can not be entirely ruled out. For instance, the magnetic properties of the lower crust are still poorly understood and may significantly vary along axis. At segment ends, where tectonic extension dominates and magmatic activity is relatively weak, serpentinite bodies within the lower crust may play a role in the along-axis magnetic structure of slow spreading ridges [Pockalny et al., 1995].

What does the off-axis decay in magnetization represent? Placing the CAMH in a broader context, we examine how magnetization decays with age. This decay has long been attributed to low-temperature alteration of titanomagnetite to titanomaghemite within the source layer [Johnson and Merrill, 1973; Prévot and Grommé, 1975]. Previous studies on the MAR concluded that the apparent magnetization decay rate is characterized by an exponential time constant of $500 \mathrm{kyr}$ [Johnson and Atwater, 1977; Macdonald, 1977]. They acknowledge that this value is dependent on the width and stationarity of accretion, and is therefore an upper bound to the contribution of low-temperature alteration. Recent evidence from the fast spreading East Pacific Rise shows that the decay rate is much faster, with a time constant of $20 \mathrm{kyr}$ [Gee and Kent, 1994]. As discussed previously, the apparent exponential time constant that is an overall best fit to the deep tow profiles in this study is $150 \mathrm{kyr}$, a value intermediate between those of previous studies. The variability observed between these studies may be due to further contributions from spatially variable processes, such as along-axis variation in intrusive accretion as crust is transported off-axis, the overlap of individual volcanic flows, or variations in the amount of crust exposed to seawater due to faulting and fissuring. Because of the trade-off between magnetization decay and width of the NVZ, our value of 150 kyr most likely places an upper bound to the width of volcanic emplacement and implies that spreading is focused at a scale of less than $2 \mathrm{~km}$. 
The 150-kyr decay rate fits all the profiles well, except for the western flank of segment 1, which is best fit by a 1.5-Myr time constant. The segment at this location, however, is slightly anomalous in that a large unaltered block of sedimented, but wellpreserved hummocky terrain with an extremely high magnetization sits outside the western valley wall (Figure 3-6b). In the sea surface magnetization map, it correlates with a knob of high magnetization bulging off-axis to the west at $24^{\circ} 19^{\prime} \mathrm{N}$ (Figure 33). The high magnetization would seem to indicate that it is quite young and probably a block of crust that has been transposed by a recent propagation event between the two segments. The profile crossing near the center of segment 1 is well modeled by the 150-kyr decay rate (Figure 3-6c). Modeling the magnetization decay across the nodal deep and inner corner high is a difficult task because of an off-axis relict neovolcanic ridge that is strongly magnetized (Figure 3-6d). The high magnetization over this ridge suggests the presence of fresh lavas. Forward modeling shows that the relict ridge causes the magnetization in that area to be higher than that predicted for a simple exponential decay.

In summary, the exponential magnetization decay rate in this area is best fit by a time constant of $150 \mathrm{kyr}$. This value reflects a combination of the decay in magnetization due to low-temperature alteration and the spatial averaging of the crustal accretionary process. The low-temperature alteration decay rate is likely to be much faster and may be as rapid as has been suggested for the EPR [Gee and Kent, 1994]. The varied estimates of decay rate along the mid-ocean ridge system are most likely due to a spatially varying crustal accretion process.

\subsubsection{Axial Valley Wall Magnetization Lows}

In all but the southern profile, zones of near-zero magnetization flank the NVZ within the Brunhes epoch, and several of these zones suggest negative values. If they truly mark the location of negatively magnetized crust, then it must be through either the capture of older crust or a geomagnetic reversal event within the Brunhes. Morpho- 
logic expressions that indicate segment propagation have been observed in this region [N. Seama, personal communication, 1995], but it is unlikely that the rift valley walls in each profile contain captured crust. Paleointensity variations or actual reversal events must correlate with time across all profiles. Drastic differences in the proximity of these zones to the CAMH show that such a geomagnetic event can not be responsible for their occurrence.

Another possibility is that a sudden drop to zero magnetization causes the inversion solution to overshoot into negative values. Although geological constraints significantly reduce the ambiguity of locating a zero level, magnetic anomalies alone contain information only on magnetization contrasts. If we consider the difficulty in retaining nonzero DC magnetization levels and linear trends in the Fourier method and the limiting assumption of two dimensionality, it is reasonable that these zones may represent nonmagnetic sections of crust. We believe that the drastic change in magnetization associated with the valley walls is geologically significant and that overshoot by the inversion process is the best interpretation of the predicted negative values at those locations.

Rift valley walls are associated with a high degree of faulting and fissuring. Increased seawater penetration and tectonic disruption in these zones is likely to cause intense magnetic source destruction in the form of enhanced hydrothermal alteration and mechanical randomization of the crustal fabric within disturbed zones, such as talus piles below fault scarps. Hydrothermal alteration alone has been shown to reduce the magnetization of seafloor basalts by at least an order of magnitude [e.g., Wooldridge et al., 1987]. If fault throw is significant, physical thinning of the magnetic source layer may also result. In the northern two profiles, these zones are several kilometers from the neovolcanic axis and therefore allow low-temperature alteration to substantially lower the magnetization at the flanks of the CAMH (Figures 3-6a and $3-6 \mathrm{~b})$. In profile 2 , the model inversion solution for the eastern valley wall crosses over into negative values (Figure $3-6 \mathrm{~b}$ ). This illustrates the argument that negative 
values predicted along the flanks of the CAMH are inversion overshoots due to the sharp amplitude transition and profile geometry. Profile 3 crosses segment 1 just south of its narrowest point. Here, the zones of intense demagnetization are very close to the volcanic axis, especially in the west. In this case, the magnetization peak does not correctly predict the location of most recent volcanic construction because the adjacent zone of source destruction to the west shifts the peak eastward by about $450 \mathrm{~m}$. In contrast, the inside corner wall in the southern profile is approximately $10 \mathrm{~km}$ from the NVZ. At that distance, the model magnetization has nearly dropped to its background level of $4 \mathrm{~A} / \mathrm{m}$. The magnetic reversal near the base of the valley wall may also help to explain the apparent absence of a zone of source destruction. If one exists, it would be partially masked by the reversal event and would have a much more subtle effect on the inversion solution than if it occurred closer to the axis, where the associated magnetization contrast would be more extreme.

Note the higher magnetization outside the inner valley walls (e.g., $8 \mathrm{~km}$ from the axis in Figure 3-5a and $-10 \mathrm{~km}$ in Figure 3-5b). One possible explanation is that crustal material is remagnetized as it passes into the rift mountains, either through chemical remanence [e.g., Raymond and LaBrecque, 1987] or by the formation of new magnetic minerals (e.g., serpentinization or off-axis volcanism). Little evidence has been found to support any type of remanence off-axis other than thermal [Beske-Diehl, 1989], and the lack of evidence for volcanism outside the rift valley, especially within the rift mountains (often $>10 \mathrm{~km}$ from the neovolcanic zone), makes this hypothesis unlikely. Serpentinites are typically associated with fault scarps, so it is difficult to separate the effects of serpentinization on the magnetic field from those of enhanced alteration and crustal disruption. Harrison [1987] reports that the magnetization of serpentinized peridotites can be quite high $(>6 \mathrm{~A} / \mathrm{m})$. However, serpentinites are usually recovered within $30 \mathrm{~km}$ of ridge axis discontinuities [Cannat, 1993] and thus cannot explain this return to higher magnetizations within the rift mountains near segment centers. The only other plausible explanation for these magnetization 
highs at the outer flanks of the axial valley lows is that sections of crust have been transported out of the median valley relatively undisturbed. TOBI side-scan sonar data show abundant evidence for undisrupted terrains between major faults in the median valley flanks that preserve morphologies similar to those seen in the young valley floor.

On the basis of our observations, we propose a cyclic faulting process similar to that described by Ballard and Van Andel [1977] and Macdonald and Luyendyk [1977] for the MAR at $37^{\circ} \mathrm{N}$ and more recently proposed by Kappel and Ryan [1986] and Tivey and Johnson [1987] for the Juan de Fuca ridge and by Bicknell et al. [1987] for the East Pacific Rise. The basic assumption of this model is that large, unaltered crustal blocks are lifted out of the rift valley by adjacent zones of faulting (Figure 3-7). This process contrasts with models in which the crust acts as a conveyor belt that moves across broad, stationary fault zones within the valley walls, because it predicts that portions of crust travel out of the rift valley tectonically unscathed and therefore magnetically preserved. We suggest that as the crust moves away from the zone of emplacement, it begins faulting and fissuring, which accelerates source destruction at those locations (Figure 3-7). Strain is localized in these zones until they are too distant from the NVZ to accommodate stresses near the ridge axis. At that point, a new zone, closer to the axis, begins failing. Previous studies have suggested that the most likely location for the genesis of this new fault zone is either on the flanks of the volcanic constructional ridge [Ballard and Van Andel, 1977] or within the ridge itself [Kappel and Ryan, 1986; Tivey and Johnson, 1987]. This faulting model predicts an oscillatory pattern of highs and lows for the cross-axis magnetization and areas of intense source destruction flanking the zone of emplacement (Figure 3-7). Yet it does not preclude large sections of unaltered crust from being transported into the rift mountains. This process is what Zonenshain et al. [1989] may have had in mind when they speculated that the relict volcanic ridge in the nodal deep will grow into a new inside corner high and that present-day tectonic activity has shifted to the 

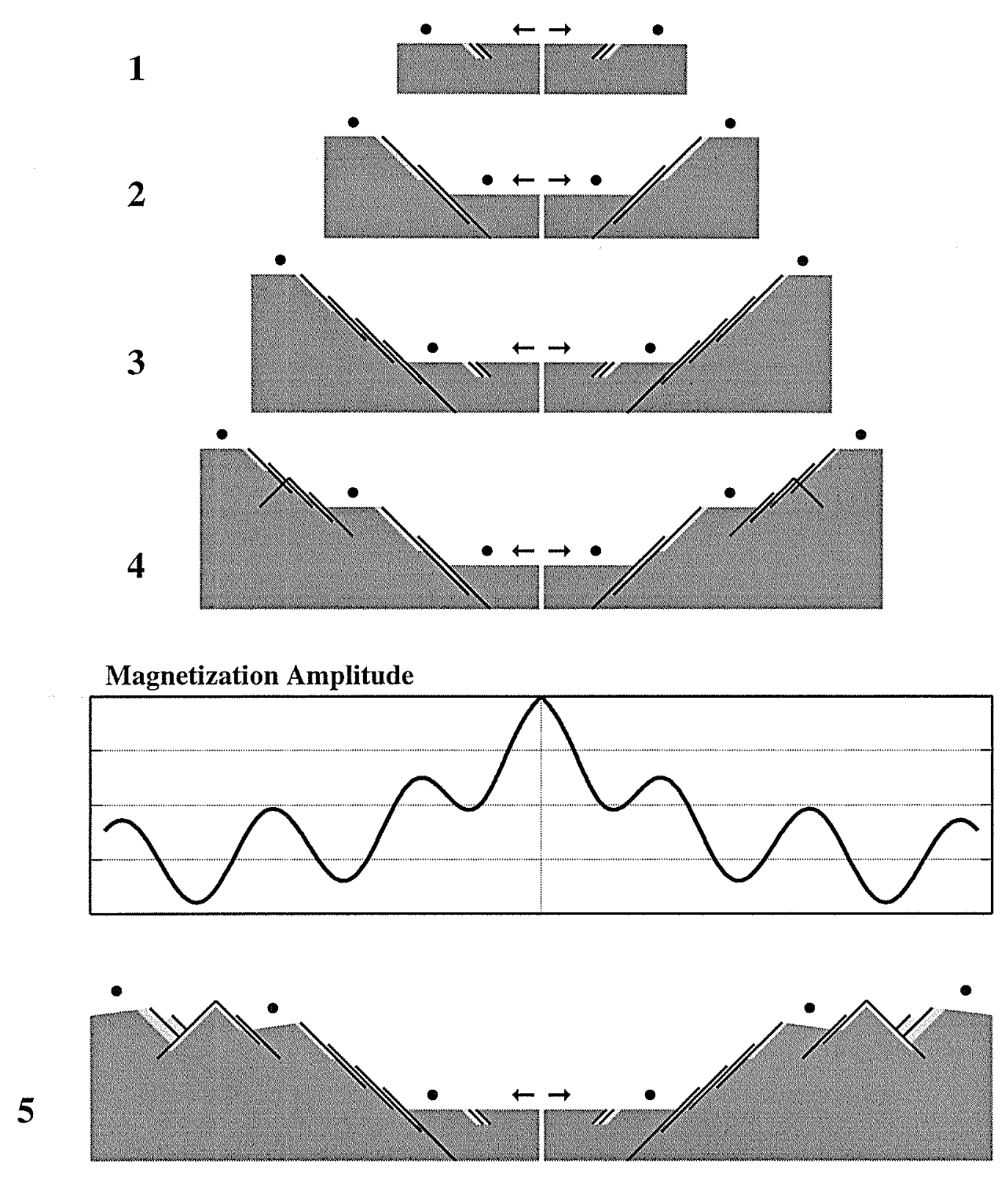

Figure 3-7: A sequential depiction of the cyclic faulting model proposed as a mechanism for magnetic source destruction. Bullets mark unaltered sections of crust. As material spreads away from the zone of emplacement (1), the brittle crust begins faulting and fissuring (2), which accelerates source destruction at those locations. Strain is localized in these zones until they can no longer accommodate stresses near the ridge axis, at which point a new zone, closer to the axis begins failing ( 3 and 4 ). This faulting model introduces an oscillatory pattern into the magnetization, which exponentially decays as a result of low-temperature alteration. The model predicts areas of intense source destruction flanking the zone of emplacement, yet it allows large sections of relatively unaltered crust to be transported into the rift mountains (5). 
intervening basin. Note that this cyclic faulting model is not coupled to any single accretionary process. The faulting process is more likely a function of crustal strength and spreading rate rather than volcanic episodicity. In two dimensions, one might envision this cyclicity manifesting itself as linear magnetic lows flanking the CAMH. Since different parts of the rift valley are not necessarily at the same stage within the faulting cycle, these lows would not always be the same distance from the NVZ.

Recent geologic models of structural processes at slow spreading ridges [Mutter and Karson, 1992; Tucholke and Lin, 1994] have suggested that a combination of detachment faulting and episodic magmatic as well as amagmatic extension is responsible for both the anomalously shallow crust on the inside corner of large discontinuities and the strong heterogeneity of crust off-axis. They propose that a low-angle detachment fault strips the extrusive layer from the inside corner and that during amagmatic periods, lower crust and upper mantle rocks are exposed in a relatively short period of time. The extrusive layer is thought to be the dominant source of magnetic field anomalies. Its possible absence at the inside corner is supported by the extremely weak anomaly pattern observed there in Figure 3-3. Episodic magmatic extension, in conjunction with detachment faulting, may also generate the oscillatory magnetization pattern observed in the deep tow profiles by periodically exposing ultramafics at inside corners and enhancing alteration and crustal thinning at outside corners during periods of tectonic extension. Further studies are needed to determine the extent that these models affect the magnetic signature of crust at slow spreading ridges.

\subsection{Conclusions}

We have examined four deep tow magnetic profiles obtained by the TOBI system across the ridge axis immediately north of the Kane transform and have studied their implications for the accretion and alteration processes at the MAR. A comparison of these profiles with sea surface data shows that deep tow magnetics offers increased 
resolution of the fine-scale magnetic structure in the upper crust. Two features are consistently observed in the inversion solutions: the central anomaly magnetization high and rift valley wall magnetization lows. In conjunction with preliminary analyses of sonar images and camera footage, as well as previous work in this area, we have constrained the volcano-tectonic evolution of oceanic crust in a slow spreading environment:

1. The CAMH accurately marks the zone of most recent crustal emplacement. Although not well resolved in sea surface data, its narrow, peaked nature in the deep tow profiles suggests that accretion is highly focused at the ridge axis. This contradicts previous models that invoke a temporal and spatial variability in crustal emplacement to account for the heterogeneity observed at the MAR.

2. Axial valley walls locate zones of magnetic source destruction due to fissuring and faulting of the upper crust. Associated magnetization lows are not resolved in sea surface data but are clearly recorded in the deep tow profiles. Magnetization highs within the rift mountains suggest that a cyclic faulting process is at work, whereby large, unaltered blocks of crust are lifted out of the rift valley by adjacent zones of faulting.

3. Along-axis variation of the CAMH on the segment scale in this survey may be largely explained by increased $\mathrm{Fe}$ and $\mathrm{Ti}$ content measured in basalts at segment ends.

4. The exponential magnetization decay constant due to low-temperature alteration has an upper bound of $150 \mathrm{kyr}$, a value intermediate to those suggested by previous studies. We suggest that the variability observed in the rate of magnetization decay is due to contributions from spatially variable processes, such as along-axis variations in off-axis intrusive accretion.

5. Our observations of fine-scale magnetic structure show that while the emplacement of crust at slow spreading ridges is highly focused, subsequent tectonic disruption and alteration at the rift valley walls degrade the crustal magnetic signal 
and may be the primary reasons for Atlantic magnetic anomaly variability.

\subsection{Acknowledgments}

Magnetic data were collected during cruise 57 of the RSS Charles Darwin by the National Environment Research Council. We thank N. K. Lawson, N. Seama, and D. K. Smith for their informative discussions. This manuscript was greatly enhanced by the helpful reviews of K. C. Macdonald, J. Gee, and W. W. Sager. Funding was provided by an Office of Naval Research fellowship (S. A. Hussenoeder), NSF grants OCE-9200905 and OCE-9204121 (M. A. Tivey and H. Schouten), and NERC GR3/7702 (R. C. Searle). Woods Hole Oceanographic Institution contribution 9160.

Hussenoeder, S. A., M. A. Tivey, and H. Schouten, Near-bottom magnetic survey of the Mid-Atlantic Ridge axis, $24^{\circ}-24^{\circ} 40^{\prime} \mathrm{N}$ : Implications for crustal accretion at a slow spreading ridge, J. Geophys. Res., 101, 22,051-22,069, 1996. Copyright by the American Geophysical Union. Reprinted with permission. 


\title{
Chapter 4
}

\section{Seismic analysis of the axial magma}

\section{chamber reflector along the southern}

\author{
East Pacific Rise from conventional
}

\section{reflection profiling}

\begin{abstract}
The thickness and internal properties of the magma sill located at the top of the axial magma chamber (AMC) along the southern East Pacific Rise (EPR) have been investigated through a combination of waveform modeling the near-vertical incidence reflections from this body and analysis of reflection amplitude variation as a function of source-receiver offset (or slowness). Our results show that the AMC reflector observed along the southern EPR is best modeled by a thin ( $<100 \mathrm{~m}$ thick) sill of partial melt $\left(V_{s} \neq 0 \mathrm{~km} / \mathrm{s}\right)$ sandwiched between higher-velocity material, and that the thickest sills are generally associated with the lowest $P$ and $S$ wave velocities. The comparatively high $P$ wave velocities and nonzero shear wave velocities inferred for this sill indicate that it is filled with partially molten magma which in some locations has a high crystal content. This may have important implications for eruption mechanisms and along-axis mixing of magma at the EPR. There is no simple relationship between morphologic indicators of magma supply (e.g., axial depth or volume) and sill thickness, depth, or velocity. Magma sill properties may be closely tied to the eruption and replenishment cycle of the AMC and thus may vary on a much shorter spatial and temporal scale than axial morphology, which reflects longer-term variations in magma supply to the ridge.
\end{abstract}




\subsection{Introduction}

The size and shape of the axial magma chamber (AMC) beneath the fast spreading East Pacific Rise (EPR) have been the subject of recent intense investigation [Detrick et al., 1987; Harding et al., 1989; Toomey et al., 1990; Vera et al., 1990; Kent et al., 1990; Caress et al., 1992; Detrick et al., 1993; Kent et al., 1993, 1994]. These studies have led to a model in which a thin $(<200 \mathrm{~m}$ ), narrow (typically $<1 \mathrm{~km}$ wide) lens or sill of magma 1-2 km below the seafloor overlies a zone of partial melt in the midcrust that is in turn surrounded by a broader low-velocity volume (5-10 km wide) extending to the base of the crust [Sinton and Detrick, 1992]. Although the magma sill at the roof of this low-velocity body may be volumetrically quite small, it is thought to play a key role in the availability and composition of magma at the rise axis [Sinton and Detrick, 1992]. A clearer understanding of its properties and their variation along axis will provide additional insight into the genesis of oceanic crust.

The existence of a shallow magma sill beneath the EPR is known primarily from seismic reflection data collected along and across the rise axis. A high-amplitude "AMC reflector" from the roof of the AMC has been mapped as a relatively continuous event 1-2 km below the seafloor along large sections of both the northern [Detrick et al., 1987] and southern [Detrick et al., 1993] EPR. The large normal-incidence reflection coefficients (as high as 0.2) associated with this event [Barth et al., 1987; Vera et al., 1990] and its often phase-reversed waveform relative to the seafloor reflection [Barth et al., 1987; Detrick et al., 1987; Vera et al., 1990] are consistent with the large negative impedence contrast expected between magma and overlying crustal rocks. Migration and forward modeling of diffraction hyperbolae in unmigrated cross-axis reflection profiles, generated by the abrupt termination of the sill, indicate a typical sill width of 500-1000 m [Kent et al., 1990, 1993, 1994]. Estimates of the thickness of this body are not well constrained. Tomographic velocity models [Toomey et al., 1990, 1994] and seismic attenuation studies [Wilcock et al., 1992] place an upper limit of 1-2 km on the thickness of any largely molten, midcrustal body beneath the EPR. 
The identification of a second, deeper reflection in waveform studies of the AMC indicates the presence of a thin magma sill with a thickness of only a few hundred meters or less [Harding et al., 1989; Kent et al., 1993; Detrick et al., 1993]. Both the waveform and amplitude of reflections from such a thin magma sill are highly sensitive to small changes in thickness and melt fraction [Kent et al., 1990; Phipps Morgan et al., 1994].

The internal properties (e.g., temperature, crystal content, composition) of this magma sill and their spatial and temporal variation along axis are still poorly known. On the basis of plane wave reflection coefficient modeling of an expanding spread profile (ESP) obtained along the northern EPR at $13^{\circ} 13^{\prime} \mathrm{N}$, Harding et al. [1989] inferred the presence of a partially molten magma sill (i.e., $V_{s} \neq 0 \mathrm{~km} / \mathrm{s}$ ) or a double transition from solid to mush to melt at the roof of the AMC. In contrast, an amplitude variation with offset analysis of the AMC event at $9^{\circ} 30^{\prime} \mathrm{N}$ led Vera et al. [1990] to suggest the presence of a fully molten magma body (i.e., $V_{s}=0 \mathrm{~km} / \mathrm{s}$ ). The variation in amplitude and waveform of the $\mathrm{AMC}$ reflector observed along the EPR, including its disappearance along some sections of the ridge, is further evidence that the AMC exhibits spatial and/or temporal variability both in size and melt content.

In this paper, we examine multichannel seismic reflection data to investigate the properties of the AMC reflector along the southern EPR. Through the use of waveform modeling, we show that a thin $(<100 \mathrm{~m})$ magma sill is present near the top of the AMC. From the analysis of the variation in reflection amplitude as a function of slowness we demonstrate that this sill is not entirely molten. Our results show that its thickness and crystallinity vary along the ridge in a way that is not simply related to other indicators of magma supply, such as axial depth or volume. We speculate that the properties of the magma sill are closely tied to the eruption and replenishment cycle of the AMC and vary on a much shorter spatial and temporal scale than axial morphology, which reflects the longer-term supply of magma to the ridge. 


\subsection{Data Collection and Analysis}

In 1991, TERA, a two-ship, three-institution multichannel experiment, investigated the seismic structure of the ultrafast spreading East Pacific Rise axis south of the Garrett transform [Detrick et al., 1993; Kent et al., 1994; Mutter et al., 1995]. Data were acquired aboard the R/V Maurice Ewing of the Lamont-Doherty Earth Observatory using a 160-channel, 4-km-long digital streamer with a 25-m group separation. For lines north of $17^{\circ} 20^{\prime} \mathrm{S}$ the seismic source was a 20 -gun, 8385 cubic inch air gun array fired every $20 \mathrm{~s}$, yielding a shot spacing of $50 \mathrm{~m}$ and forty-fold coverage for a nominal ship speed of 5 knots $(\sim 2.5 \mathrm{~m} / \mathrm{s})$. South of this area, port and starboard halves of the array were alternately fired every $10 \mathrm{~s}$, yielding a shot spacing of $25 \mathrm{~m}$ and eighty-fold coverage. Common depth point (CDP) reflection data along the northern EPR were collected in 1985 aboard the R/V Conrad using a 48-channel, 2.4-km-long streamer with a 50-m group separation. The shot interval was $20 \mathrm{~s}$, which yielded a $50-\mathrm{m}$ shot spacing and $\sim 24$-fold coverage.

Four locations along the southern EPR and one at $9^{\circ} 39^{\prime} \mathrm{N}$ were chosen for study (Figure 4-1 and Table 4.1). Constant offset stacks (COS) were constructed for each location by summing 50 CDP gathers in time-range $(t-x)$ space to enhance the signalto-noise ratio and band-pass filtering the traces between 5 and $40 \mathrm{~Hz}$ to remove low-frequency strumming of the streamer and spurious high-frequency noise. Data analysis consisted of the construction of common midpoint (CMP) stacks for each area of interest, estimation of the near-offset composite reflection coefficient of the AMC, waveform analysis of the near-offset AMC reflection, slant stacking of each COS into delay time-slowness space $(\tau-p)$, and forward modeling the AMC reflection amplitude versus slowness (AVS).

CMP stacks were constructed for each location by applying the appropriate normal moveout (NMO) velocities to the seafloor, layer 2A, and AMC reflections. Seafloor and postcritical reflections near the base of layer $2 \mathrm{~A}$ were muted at ranges $>3200 \mathrm{~m}$ to prevent excessive stretching that occurs in this region during the application of 


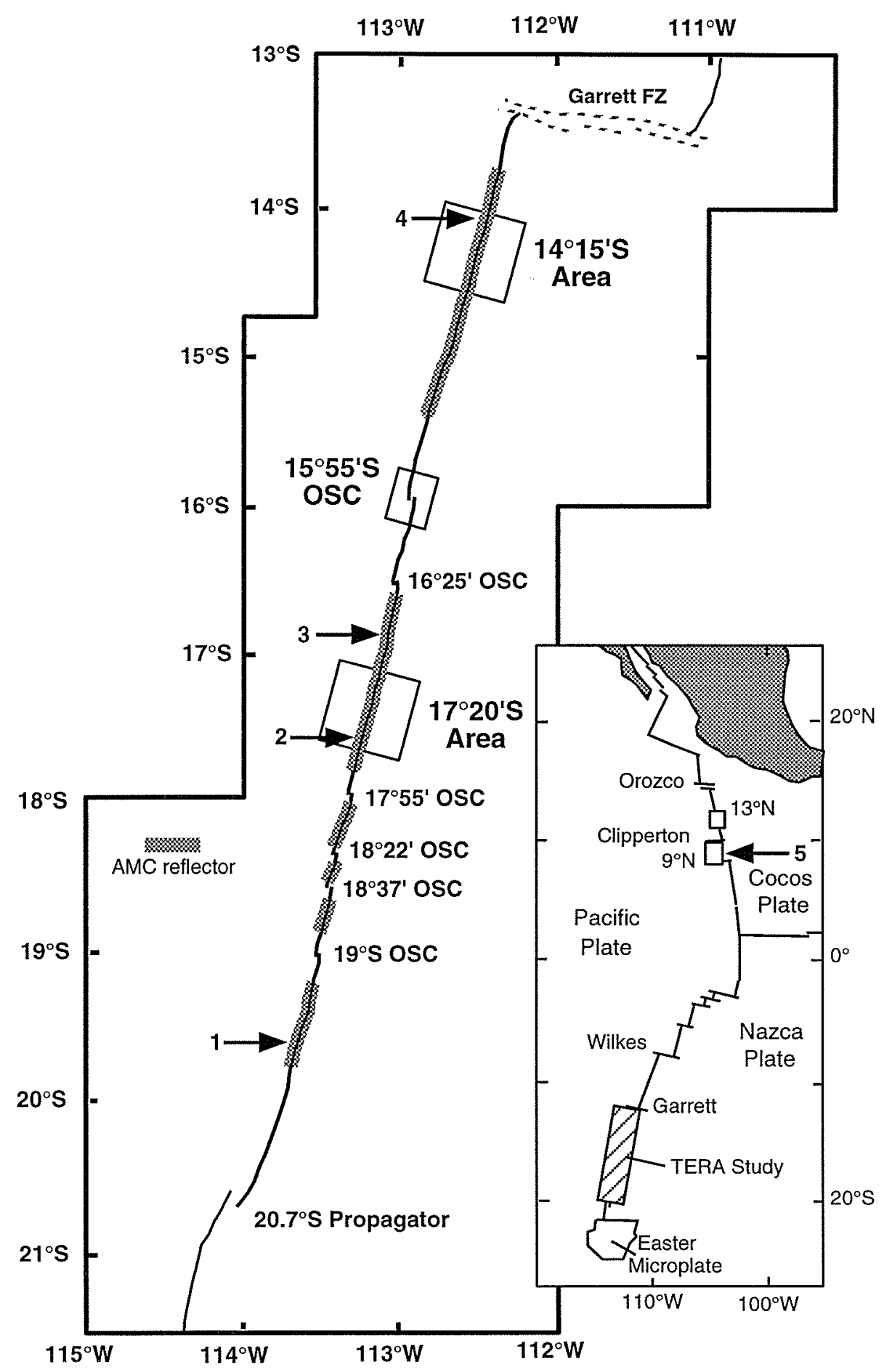

Figure 4-1: Map of the southern EPR. Major segment boundaries are indicated, and gray bands show those portions of ridge which exhibit an AMC reflection in the along-axis CDP data. Numbered arrows indicate those points along the rise axis from which data were extracted and analyzed in this study. Excerpted with permission from Detrick et al. [1993]. Copyright 1993 American Association for the Advancement of Science. 
Table 4.1: Summary of Results

\begin{tabular}{cccccc}
\hline Location & Line \# & CDPs $^{*}$ & Latitude & Longitude & Axial Depth \\
\hline 1 & SEPR03 & $5,330-5,380$ & $19^{\circ} 36^{\prime} \mathrm{S}$ & $113^{\circ} 35^{\prime} \mathrm{W}$ & $2791 \mathrm{~m}$ \\
2 & SEPR09 & $6,550-6,600$ & $17^{\circ} 33^{\prime} \mathrm{S}$ & $113^{\circ} 14^{\prime} \mathrm{W}$ & $2613 \mathrm{~m}$ \\
3 & SEPR35 & $6,650-6,700$ & $16^{\circ} 52^{\prime} \mathrm{S}$ & $113^{\circ} 05^{\prime} \mathrm{W}$ & $2629 \mathrm{~m}$ \\
4 & SEPR45 & $18,950-19,000$ & $14^{\circ} 05^{\prime} \mathrm{S}$ & $112^{\circ} 30^{\prime} \mathrm{W}$ & $2635 \mathrm{~m}$ \\
5 & NEPR41 & $10,310-10,360$ & $9^{\circ} 39^{\prime} \mathrm{N}$ & $104^{\circ} 16^{\prime} \mathrm{W}$ & $2547 \mathrm{~m}$ \\
\hline
\end{tabular}

\begin{tabular}{cccccc}
\hline Location & $\begin{array}{c}\text { AMC } \\
\text { Depth }\end{array}$ & $R_{A M C}$ & $\begin{array}{c}\text { Sill } \\
V_{p}(\mathrm{~km} / \mathrm{s})\end{array}$ & $\begin{array}{c}\text { Sill } \\
V_{s}(\mathrm{~km} / \mathrm{s})\end{array}$ & $\begin{array}{c}\text { Sill } \\
\text { Thickness }(\mathrm{m})\end{array}$ \\
\hline 1 & $472 \mathrm{~ms}$ & $-0.26 \pm .05$ & $3.50 \pm .4$ & $1.15 \pm .2$ & $48 \pm 10$ \\
2 & $492 \mathrm{~ms}$ & $-0.07 \pm .05$ & $4.65 \pm .4$ & $2.30 \pm .3$ & 9 or $32 \pm 10$ \\
3 & $520 \mathrm{~ms}$ & $-0.23 \pm .05$ & $3.75 \pm .2$ & $1.50 \pm .2$ & $60 \pm 10$ \\
4 & $448 \mathrm{~ms}$ & $-0.20 \pm .05$ & $4.05 \pm .5$ & $1.40 \pm .5$ & $50 \pm 10$ \\
5 & $624 \mathrm{~ms}$ & $-0.23 \pm .05$ & $3.80 \pm .4$ & $1.45 \pm .5$ & $82 \pm 10$ \\
\hline
\end{tabular}

${ }^{*} \mathrm{CDP}$, common depth point

NMO. Migration was not applied to these low-topography stacked sections.

An estimate of the near-offset AMC reflection coefficient $\left(R_{\mathrm{AMC}}\right)$ is obtained by first treating the seafloor reflectivity as a ratio of amplitudes $(W)$ between the seafloor and seafloor multiple event corrected for geometric spreading and water column attenuation:

$$
R_{\mathrm{SF}}=\frac{-2\left(W_{\mathrm{SFM}}\right)}{W_{\mathrm{SF}}} \exp \left(\frac{|\omega| T_{w}}{2 Q_{w}}\right)
$$

$\mathrm{AMC}$ reflectivity is then

$$
R_{\mathrm{AMC}}=\frac{W_{\mathrm{AMC}}}{W_{\mathrm{SF}}} \frac{R_{\mathrm{SF}}}{1-R_{\mathrm{SF}}} \frac{Z_{w}+Z_{c}}{Z_{w}} \exp \left(\frac{|\omega| T_{c}}{2 Q_{c}}\right)
$$

$Z_{w}$ and $Z_{c}$ are two-way travel distances, and the exponents are inverses of the intrinsic attenuation associated with the water column and crust, respectively. Attenuation depends on total time spent within each medium $(T)$, frictional energy lost per cycle $(Q)$ [O'Connell and Budiansky, 1978], and angular frequency $(\omega)$. Equation 4.1 assumes the seafloor and AMC roof to be the only interfaces [Brocher and Phinney, 
1981]. In reality, the upper crust consists of numerous attenuative structures that, if not accounted for, will result in an underestimate of the AMC reflection coefficient. Most of the attenuation occurs within layer $2 \mathrm{~A}$, where $Q$ may have values as low as 10 [Wilcock et al., 1992; Christeson et al., 1994b]. We therefore incorporate a 125-mthick layer 2 A with a $Q$ of 15 overlying "normal" crustal material with a $Q$ of 90 into our forward models.

Near-offset AMC waveforms were modeled by a delay time transmission/reflection method that calculates the composite waveform returning from a thin lens of magma [Meissner and Meixner, 1969; Juhlin and Young, 1993]. Reflection coefficients were calculated for plane waves [Aki and Richards, 1980], given the elastic properties of the melt lens and surrounding rock. Delay times were computed as a function of slowness. All intralens multiples and $P$-to- $S$ conversions were included. Model waveforms were then compared to observed near-offset AMC reflections using the sum of the squared residuals between the waveforms as a fitting parameter.

Transforming data from arrival time-range $(t-x)$ space into delay time-slowness $(\tau-p)$ space aids interpretation by reducing each trace to a single plane wave "experiment", thereby separating reflections from different interfaces and unraveling complicated triplications [Kappus et al., 1990]. The quasi-three-dimensional "2-D+" method developed by Chapman [1978] was chosen over the fully three-dimensional HOP technique of Henry, Orcutt, and Parker [Henry et al., 1980] because of its reduced sensitivity to frequency content, aliasing, and range limits [Kappus et al., 1990]. Although computationally similar to the two-dimensional inverse radon transform [Chapman, 1981], it is derived by assuming a three-dimensional, point source geometry.

To test the 2-D+ transform's ability to retain the correct AMC amplitude pattern in a typical along-axis CDP geometry, a synthetic set of $t-x$ seismograms for a representative crustal velocity model overlying a molten half-space (Figure 4-2a) was first generated using the enhanced reflectivity method of Kennett $[1980,1983]$ and transformed into $\tau-p$ space (Figure 4-2d). The AMC reflection amplitude was then 
a) Input Crustal Velocity Model

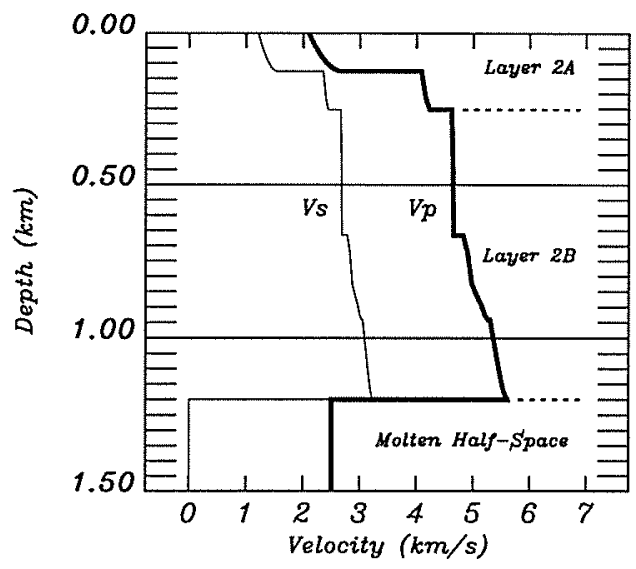

b) Estimated AMC Amplitude

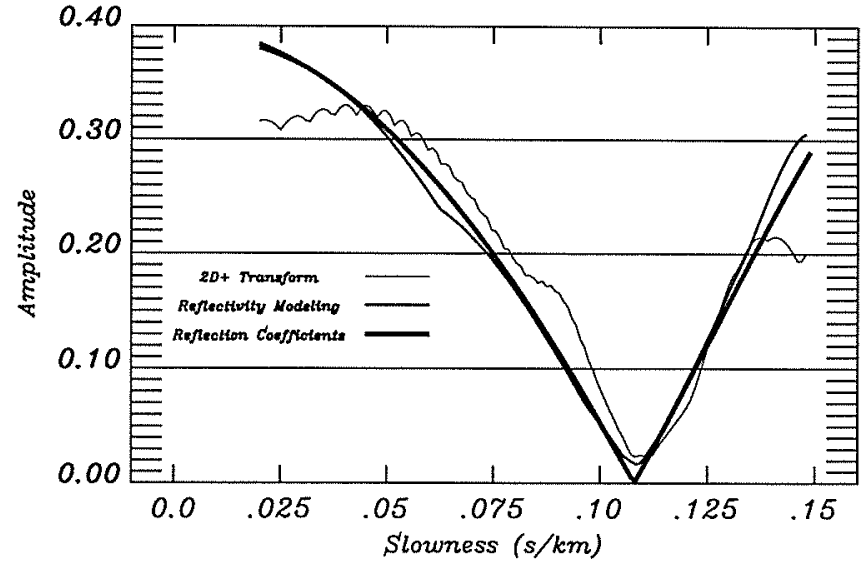

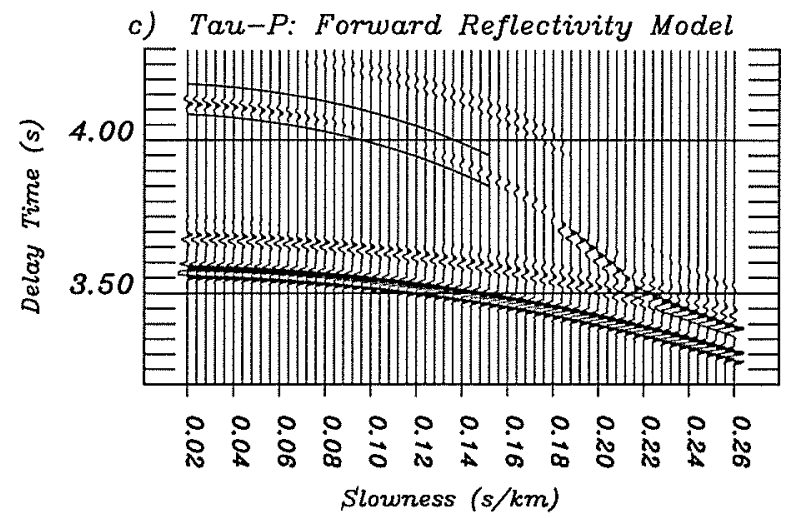

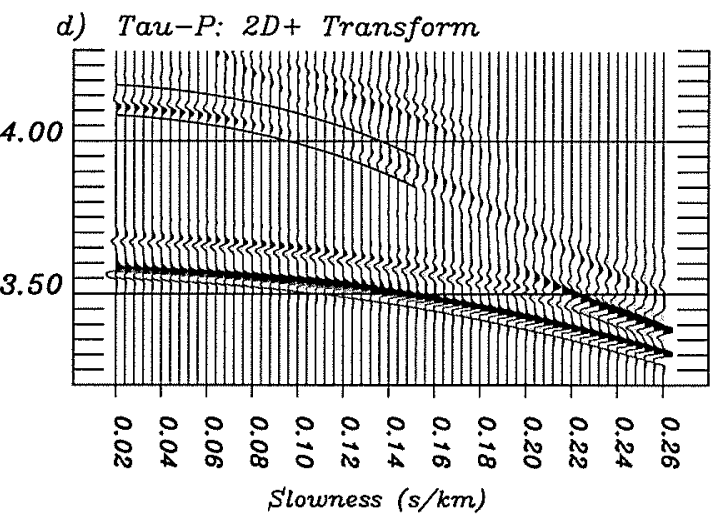

Figure 4-2: Analysis of the 2-D $+\tau-p$ transform method for a CDP experimental geometry. Using a modified velocity-depth model for (a) the EPR at $17^{\circ} 09^{\prime} \mathrm{S}$ [Detrick et al., 1993], an AMC amplitude plot (b) was constructed by generating $\tau$ - $p$ seismograms through (c) direct calculation in $\tau$ - $p$ space, using the reflectivity method of Kennett [1980, 1983], and (d) transforming a set of synthetically generated $t-x$ reflectivity seismograms between 0.263 and $4.263-\mathrm{km}$ range into $\tau-p$ space by the $2-\mathrm{D}+$ method. Parallel curves in the $\tau-p$ seismograms outline the window within which the maximum amplitude was calculated. The 2-D+ method is able to reproduce the phase inversion near $0.11 \mathrm{~s} / \mathrm{km}$ and the rapid amplitude decay toward this slowness.

calculated as a function of slowness and compared (Figure 4-2b) to the theoretical reflection coefficients for the rock/melt interface and the AMC amplitude from seismograms calculated directly (Figure 4-2c) in $\tau-p$ space. The transformed data accurately locate the phase inversion at $0.11 \mathrm{~s} / \mathrm{km}$ and reproduce the falloff in amplitude toward this slowness. The deviation at ray parameters less than $0.05 \mathrm{~s} / \mathrm{km}$ is attributed to approximations within the transform and the lack of data at small to negative ranges 
[Kappus et al., 1990]. Anomalous amplitudes at large slownesses are also generated if data at large ranges are not available. A 4-km streamer can generally record AMC reflections at the EPR out to a slowness of $0.15 \mathrm{~s} / \mathrm{km}$, and a $2.4-\mathrm{km}$ streamer can record AMC reflections out to $0.1 \mathrm{~s} / \mathrm{km}$. The close correlation between the amplitude curves in Figure 4-2b strongly suggests that the effect of interfaces within the upper crust on the amplitude characteristics of the AMC is negligible and that the AMC event can be studied by modeling the seismic response of the magma sill alone.

Forward models of the AMC event were calculated in the $\tau-p$ domain using the enhanced reflectivity method of Kennett [1980, 1983] and then convolved with a source waveform derived from near-offset seafloor reflections in the data. The resulting synthetic seismograms then included the effects of surface interaction in the vicinity of the source and receiver. Models consisted of a solid half-space above and below a magma lens of varying thickness. Model parameters included attenuation properties, compressional and shear wave velocities, density, sill thickness, and host rock properties.

AMC reflection amplitudes were calculated within a window encompassing the corresponding ellipsoidal travel time curves in $\tau-p$ space (Figures $4-2 \mathrm{c}$ and $4-2 \mathrm{~d}$ ). The largest negative value was calculated instead of a time average within the window so as to minimize the range of slownesses that any crossing event would interfere with the amplitude measurement. By inspection of the stacked data a window length of $0.1 \mathrm{~s}$ was sufficient to include all high-amplitude energy associated with the AMC reflection. At every study location along the EPR the AMC reflection amplitude at slownesses less than $0.05 \mathrm{~s} / \mathrm{km}$ decreases as the energy becomes vertically incident. The cause of this pattern, which is too large to be sea surface ghosting and is opposite to the array response function, is not fully understood at this time. We attempt to correct for this effect by calculating the difference at small slownesses between measured seafloor reflection amplitudes at each location and modeled seafloor amplitudes. This difference is then normalized and applied to the corresponding AMC amplitudes. 


\subsection{Data}

Four data sets collected during the 1991 TERA experiment along the southern EPR [Detrick et al., 1993] were chosen for analysis (Figure 4-1 and Table 4.1). For comparison we have also analyzed data collected at $9^{\circ} 39^{\prime} \mathrm{N}$ in the 1985 experiment along the northern EPR [Detrick et al., 1987]. Figure 4-3 shows both a COS (or "supergather") and CMP stack for each of these areas. The constant offset stacks display the reflected energy associated with each crustal interface as a function of source-receiver offset, while the CMP stacks show the reflections associated with each interface along track and can be used to indicate spatial variation. Figure 4-4 shows each data set transformed into $\tau$ - $p$ space and the corresponding amplitude of the AMC reflector as a function of slowness (or ray parameter). The amplitude at the smallest slowness $(p=0.02 \mathrm{~s} / \mathrm{km})$ has been set to the near-normal incidence composite reflection coefficient $\left(R_{\mathrm{AMC}}\right)$ determined for that study location using Equation 4.2. This provides a baseline for comparison with model results.

Several sections of rise axis between $19^{\circ} 36^{\prime} \mathrm{S}$ and $19^{\circ} 34^{\prime} \mathrm{S}$ on line SEPR03 were initially analyzed, all of which yielded AMC thicknesses within $2 \mathrm{~m}$ of each other and exhibited a variation in AMC velocity of less than $0.2 \mathrm{~km} / \mathrm{s}$. We therefore show only one data set from this area, that of $19^{\circ} 36^{\prime} \mathrm{S}$. Here the rise axis gradually deepens by over $300 \mathrm{~m}$ toward the $20.7^{\circ} \mathrm{S}$ propagator and has a narrower, more triangular cross section than the ridge to the north [Scheirer and Macdonald, 1993]]. A very strong AMC reflector, however, is seen at a depth of $\sim 472 \mathrm{~ms}$ beneath the seafloor (Figure 4-3a) and can be traced to within $90 \mathrm{~km}$ of the propagator [Detrick et al., 1993]. The second study area is a shallow $(\sim 2.6 \mathrm{~km}$ deep), inflated section of the ridge located at $17^{\circ} 33^{\prime} \mathrm{S}$ on line SEPR09 (Figure $4-1$ ). Although the rise axis here is significantly shallower than at $19^{\circ} 36^{\prime} \mathrm{S}$, the AMC event is slightly deeper ( $\sim 490 \mathrm{~ms}$ ) and much weaker in amplitude (Figure 4-3b). Mutter et al. [1995] propose that there has been recent volcanic activity less than $15 \mathrm{~km}$ to the north, based on the anomalously shallow depth $(<900 \mathrm{~m})$ of the AMC reflector in that region. The third site we 
a)

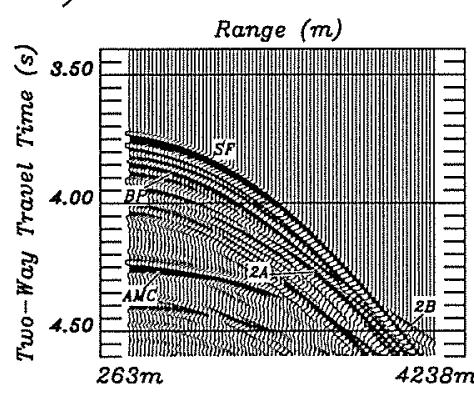

c)

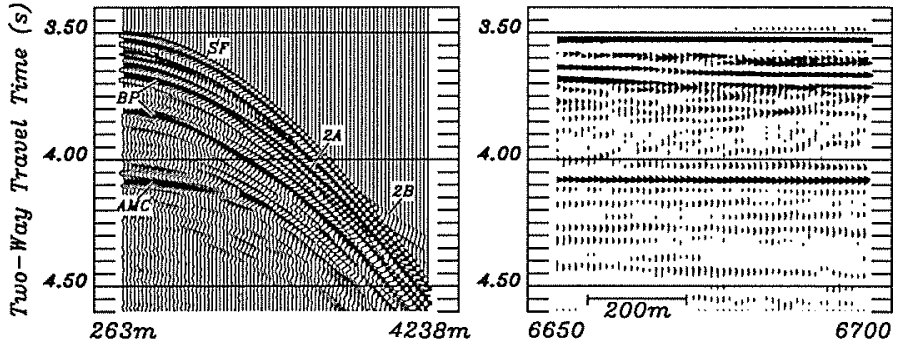

b)

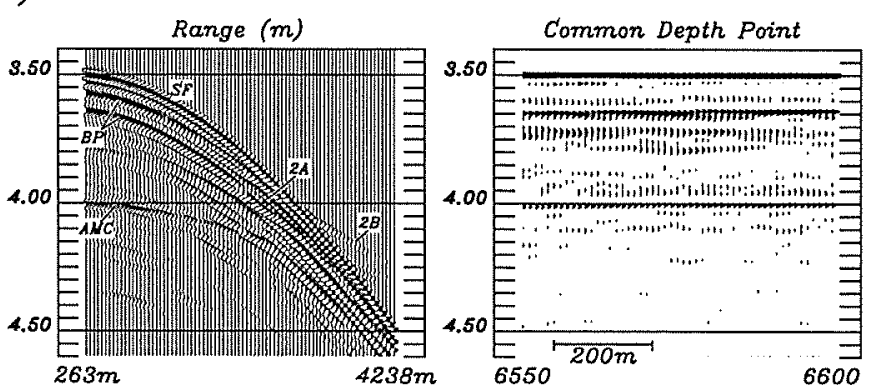

d)

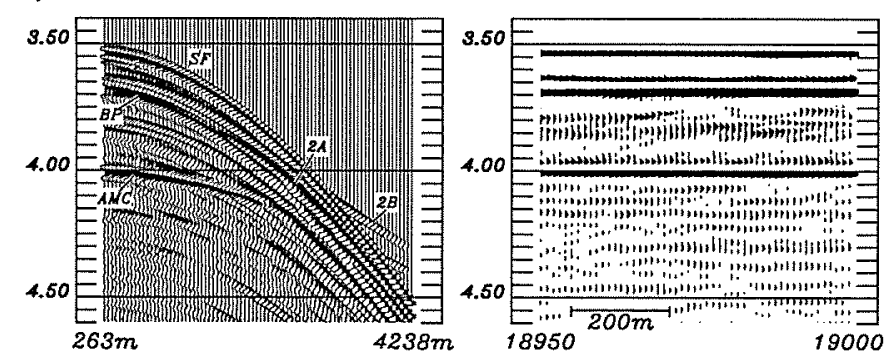

e)

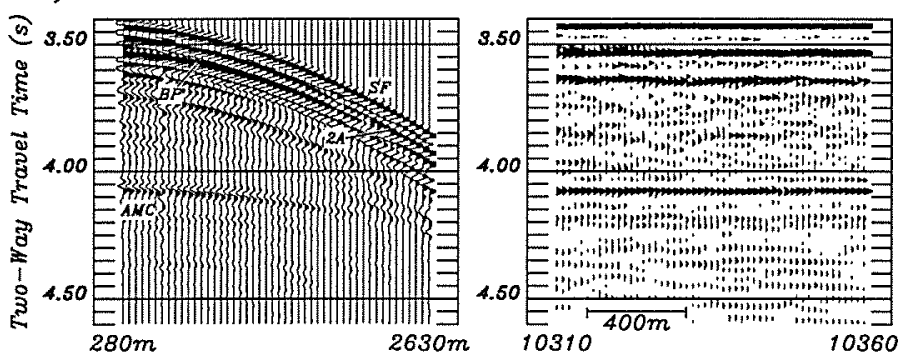

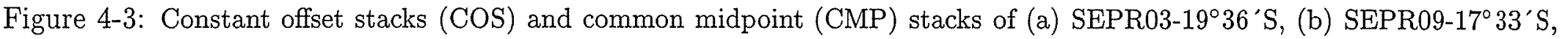

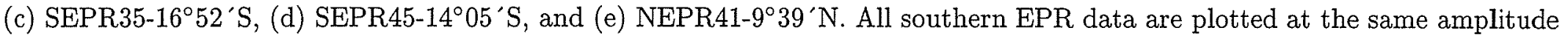
scale. Seafloor reflection (SF), bubble pulses (BP), shallow crustal refraction (2A), midcrustal refraction (2B), and AMC reflection are labeled. 
a)
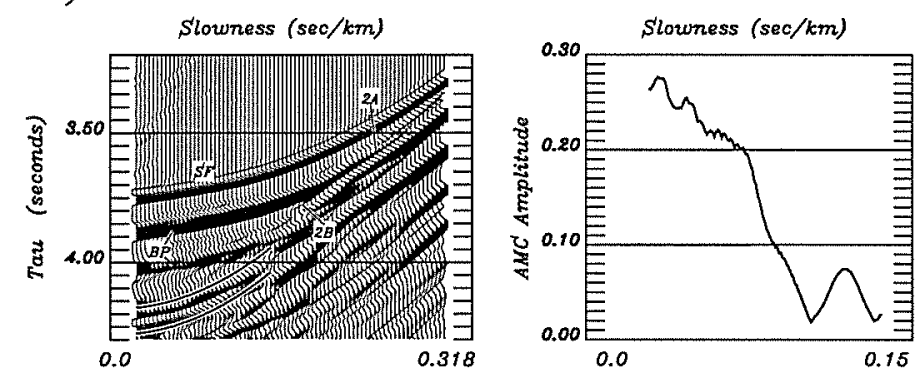

c)
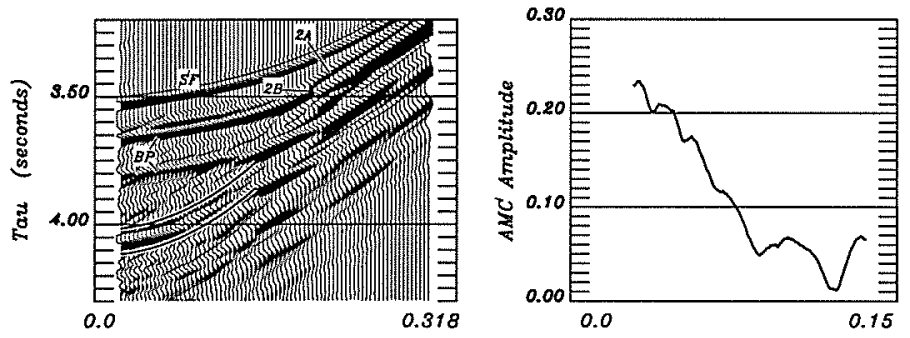

e)

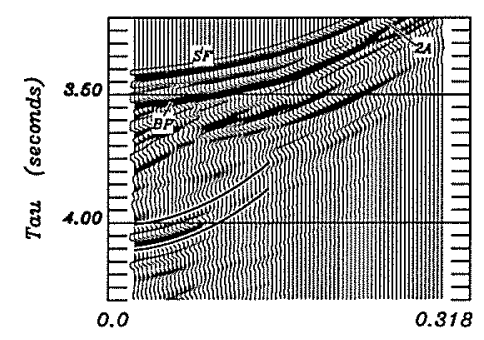

b)
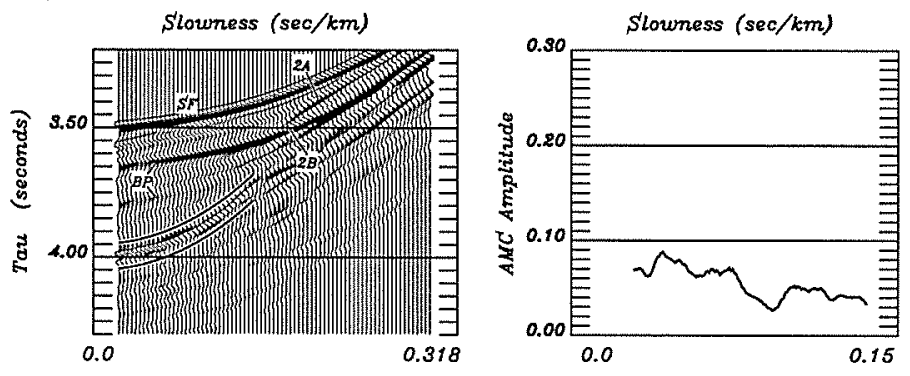

d)

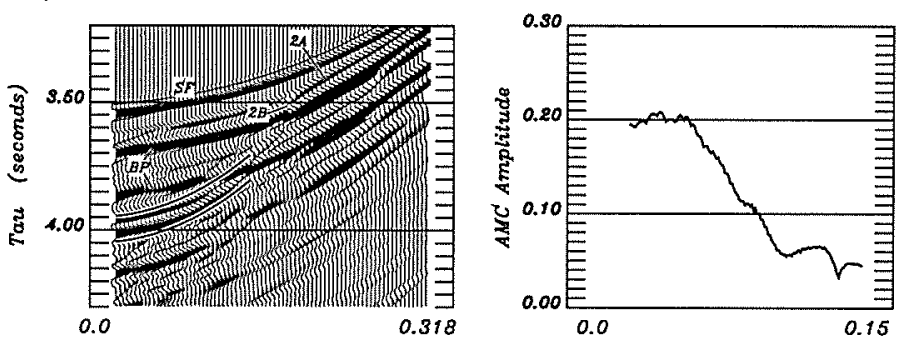

Figure 4-4: The $\tau$ - $p$ transformed seismograms and melt lens amplitude versus slowness (AVS) curves for (a) SEPR03-19 $36^{\prime} \mathrm{S}$, (b)

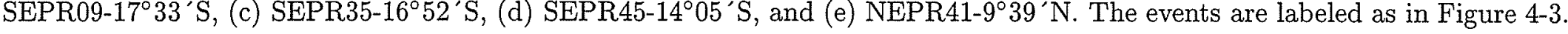
Thin curves outline windows within which the maximum negative amplitudes of the AMC event were calculated. All southern EPR $\tau$ - $p$ seismograms and all AVS curves are plotted at the same scale. 
examined lies at $16^{\circ} 52^{\prime} \mathrm{S}$ on line SEPR 35 , south of the $16^{\circ} 25^{\prime} \mathrm{S}$ overlapping spreading center (Figure 4-1). Southward from this OSC the ridge gradually becomes less narrow and blocky, and a continuous AMC event is observed, becoming quite strong near $16^{\circ} 55^{\prime} \mathrm{S}$. The AMC event here is located $\sim 520 \mathrm{~ms}$ below the seafloor, which is slightly deeper than at the two previous sites (Figure 4-3c). The northernmost area of interest on the southern EPR is located at $14^{\circ} 05^{\prime} \mathrm{S}$ on line SEPR45 (Figure 4-1) and lies on another shallow, inflated section of ridge [Scheirer and Macdonald, 1993]. At $450 \mathrm{~ms}$ below the seafloor $(\sim 1.0 \mathrm{~km})$ the strong AMC event here is the shallowest of the five study locations (Figure 4-3d). The final data set analyzed is from line NEPR41 at $9^{\circ} 39^{\prime} \mathrm{N}$ on the EPR (Figure 4-1). This area lies at the southern edge of a section of ridge that erupted in 1991 [Haymon et al., 1993], 6 years after these data were acquired. The symmetrically domed rise axis here is the shallowest of the five areas studied, but the strong AMC reflector is the deepest at $\sim 620 \mathrm{~ms}$ below the seafloor (Figure 4-3e). By modeling the shape of the AMC waveform only, Kent et al. [1993] predicted a magma sill thickness of $30 \mathrm{~m}$ at this location.

All five locations exhibit a strong, coherent AMC reflector, but the waveform of this event at near-normal incidence and its amplitude pattern as a function of slowness differ significantly among study areas. It is these differences that provide information on the variation of magma sill properties. At none of the locations is the waveform of the near-normal incidence AMC reflection clearly phase-reversed with respect to that of the seafloor reflection, as would be expected for a reflection from the top of a low-velocity half-space. Rather, its complicated pattern suggests that it is a composite reflection from multiple, closely spaced reflectors. The amplitude of the AMC reflection at near-normal incidence varies by nearly a factor of 4 between study areas, with the largest value associated with SEPR03-19 $36^{\prime}$ 'S and the lowest associated with SEPR09-17 $33^{\prime} \mathrm{S}$ (Figures 4-4a and 4-4b). Differences in the variation of amplitude with slowness are most easily observed between these two end-member cases. The AVS curve from $19^{\circ} 36^{\prime} \mathrm{S}$ exhibits an extremely high AMC reflection 
amplitude at small slownesses but abruptly drops in amplitude to less than one-tenth its near-offset value (Figure 4-4a). In contrast, the AVS curve observed at $17^{\circ} 33^{\prime} \mathrm{S}$ exhibits a low amplitude at all slownesses (Figure 4-4b). The dip in amplitude at 0.1 $\mathrm{s} / \mathrm{km}$ in SEPR09-17 $33^{\prime} \mathrm{S}$ is an interference artifact from crossing phases in the $\tau-p$ section and is not modeled in this AVS study. The AVS curves at the other three locations are intermediate to these two end-member cases (Figures 4-4c, $4-4 \mathrm{~d}$, and $4-4 \mathrm{e})$.

\subsection{Modeling and Results}

In order to understand the origin of these differences in AMC reflection amplitude and phase behavior we have forward modeled both the near-offset reflected waveforms and AVS curves. All models consist of a thin magma layer sandwiched between two half-spaces (Figure 4-5). Our approach has been to vary the velocity structure of the magma sill and host rock, as well as sill thickness, so as to optimize the fit between the observed and synthetic data. The synthetic data, generated either by reflectivity [Kennett, 1980] or the delay time transmission/reflection method [Meissner and Meixner, 1969], can be conceptually represented as a function of frequency $\omega$ and slowness $p$ :

$$
\operatorname{Model}(\omega, p)=S(\omega) \exp \left(\frac{-\omega T(p)}{2 Q}\right) R_{c}(\omega, p) D(p)
$$

The model seismograms are generated by attenuating the source waveform $S$ by an amount equivalent to propagating through the upper crust to the top of the AMC and back, where $T$ is total travel time and $Q$ is the crustal attenuation coefficient. A composite reflection term for the magma sill, $R_{c}$, that includes all multiples and wave conversions is applied to the waveform, as is a geometric spreading term $D(p)$ to account for energy loss with distance. A sum of the squared residuals between the model results and the observations was used to identify parameter values that best fit the data. It was found that waveform modeling provides tight constraints on sill 


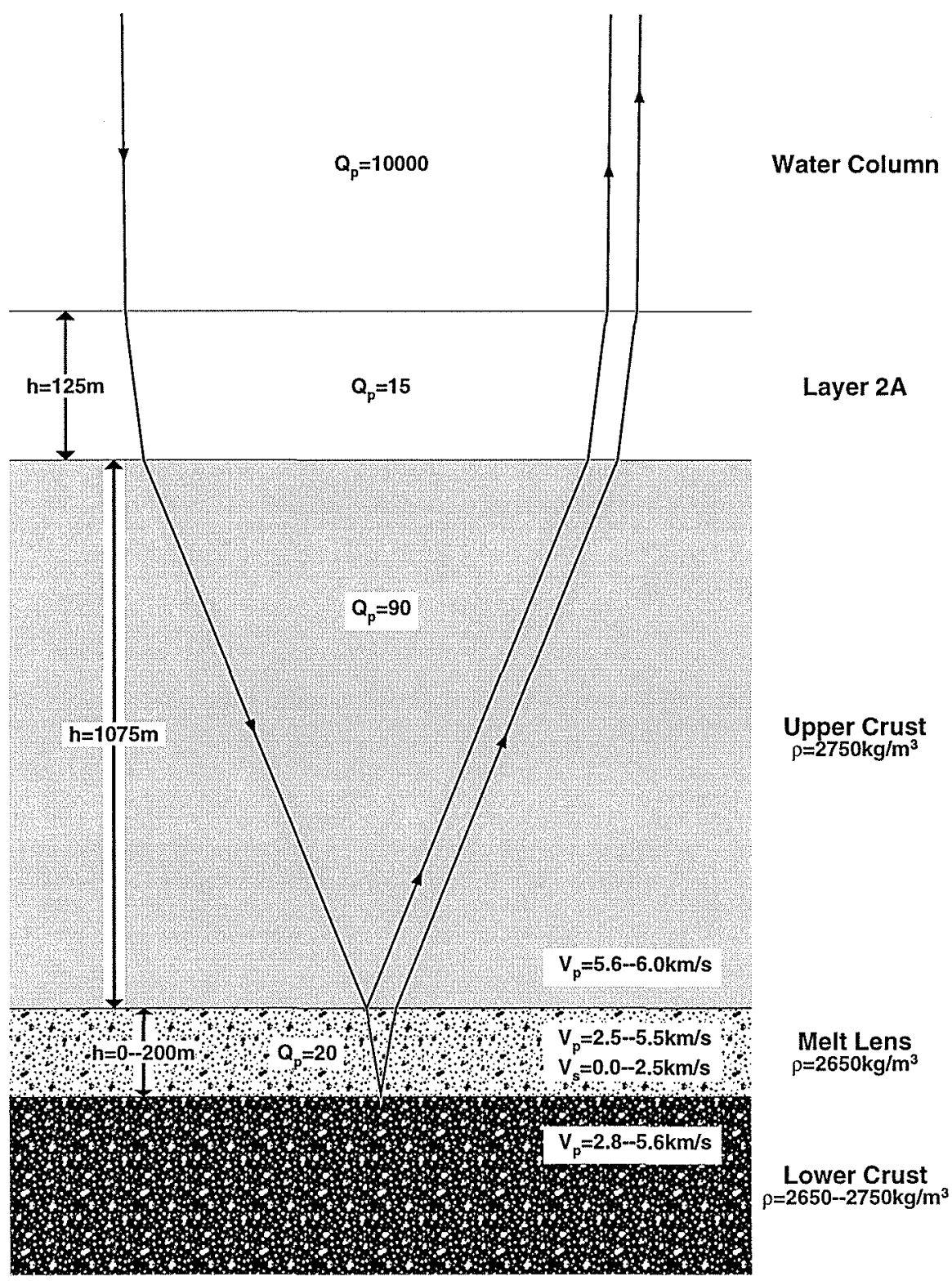

Figure 4-5: Depiction of the parameter space for both waveform and AVS modeling (see text for discussion). 
thickness and $P$ wave velocity, whereas AVS modeling best constrains $S$ wave velocity. In the following sections we discuss model parameter constraints and then summarize the results of modeling the near-vertical incidence waveforms and the AVS curves.

\subsubsection{Model Parameters}

The response of a magma sill to seismic energy is governed by numerous parameters, including sill thickness, density, compressional and shear wave velocities, and attenuation properties. The analysis methods used in this study model the seismic response of a varying magma lens sandwiched between two half-spaces. Although we show in Figure 4-2d that a realistic upper crustal structure does not cause the characteristics of the AMC reflection to deviate from theory, total attenuation within the upper crust (and water column) must still be considered. Figure 4-5 summarizes not only the range of parameters for the model magma sill and host rock but also displays the characteristics of the upper crust and water column essential to generating a realistically attenuated waveform incident on the magma sill and recorded by the receiver array.

Magma sill thickness values up to $200 \mathrm{~m}$ were modeled, as well as the end-member case of a magma half-space. $P$ and $S$ wave velocities within the magma sill were varied independently and assigned values between $2.5-5.5 \mathrm{~km} / \mathrm{s}$ and $0-2.5 \mathrm{~km} / \mathrm{s}$, respectively. This corresponds to the range of velocities measured in the laboratory for basaltic rock between its solid and purely molten state [Murase and McBirney, 1973]. The difference in density between cold (i.e., solid) and fully molten basalt is only $\sim 100 \mathrm{~kg} / \mathrm{m}^{3}$ [Murase and McBirney, 1973]. We have therefore included this density difference between the magma sill $\left(2650 \mathrm{~kg} / \mathrm{m}^{3}\right)$ and the surrounding rocks $\left(2750 \mathrm{~kg} / \mathrm{m}^{3}\right)$.

The crustal velocity immediately above the magma sill is equal to the slope of the event that is created by the convergence of the quasi-hyperbolic AMC reflection and the midcrustal refraction in a constant offset stack (Figure 4-6). Although the 


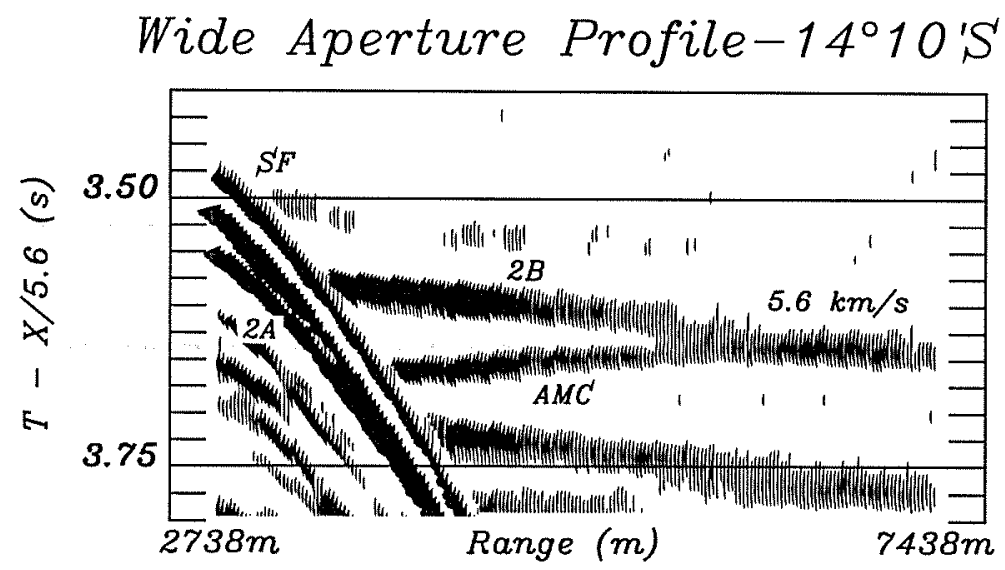

Figure 4-6: Wide-aperture profile COS from $14^{\circ} 10^{\prime} \mathrm{S}$ along the EPR. Events are labeled as in Figures 4-3 and 4-4. Horizontal event at ranges past which the layer 2B and AMC events converge shows the velocity immediately above the AMC to be equal to the reduction velocity of $5.6 \mathrm{~km} / \mathrm{s}$.

hydrophone streamers used for this analysis were too short to include this intersection, wide-aperture data from the $14^{\circ} 15^{\prime} \mathrm{N}$ study area (Figure 4-1) show the AMC lid velocity to be $5.6-5.8 \mathrm{~km} / \mathrm{s}$ over a $150-\mathrm{km}$ section of ridge (Figure 4-6). A reference value of $5.6 \mathrm{~km} / \mathrm{s}$ is therefore assigned to the lid velocity at all study locations. This is in agreement with tomographic imaging results from $9^{\circ} 30^{\prime} \mathrm{N}$ [Toomey et al., 1994] and refraction results at $14^{\circ} \mathrm{S}$ [Tolstoy et al., 1997] and $17^{\circ} \mathrm{S}$ [Detrick et al., 1993]. This value, however, is lower than typical midcrustal velocities of $6.1 \mathrm{~km} / \mathrm{s}$ [Houtz and Ewing, 1976] and lid velocities found at $13^{\circ} \mathrm{N}$ by Harding et al. [1989]. Models were calculated assuming a velocity of $6.0 \mathrm{~km} / \mathrm{s}$ in order to assess the effect of higher lid velocities on our results. It was found that this increase in lid velocity predicts a consistently thicker sill by no greater than $4 \mathrm{~m}$ and an increase in $P$ wave velocity no greater than $0.25 \mathrm{~km} / \mathrm{s}$. The increased lid velocity had little effect on sill shear wave velocity, since this parameter is primarily determined by the shape of AVS curves. Similarly, if the velocity above the magma sill is as low as $4.5 \mathrm{~km} / \mathrm{s}$, as suggested by Vera et al. [1990], sill thickness and $P$ wave velocity would be reduced and $S$ wave velocity would remain relatively unchanged. However, the analyses of Harding et al. [1989] and Vera et al. [1990] apply to the northern EPR, a spreading regime that 
differs from our study locations. In addition, Vera et al. [1990] assume the AMC event to be only the roof reflection from a purely molten body and invoke a low lid velocity largely to fit recorded travel times. The wide-aperture data available along the southern EPR place a much stronger constraint on the lid velocity (Figure 4-6). In this study, model velocities beneath the sill were varied between 2.8 and $5.6 \mathrm{~km} / \mathrm{s}$ to include the possibility of a double-step transition to a volume of partial melt. Shear wave velocities outside the sill were assumed to be equal to $V_{p} / \sqrt{3}$ (i.e., surrounding rock was modeled as a Poisson solid).

Estimates of the near-normal incidence composite reflection coefficient $\left(R_{\mathrm{AMC}}\right)$ are particularly sensitive to seismic attenuation. We have assigned a depth dependence to $Q$ in the crust above the AMC with low values $\left(Q_{p}=15\right)$ in seismic layer $2 \mathrm{~A}$ and higher values $\left(Q_{p}=90\right)$ in layer $2 \mathrm{~B}$. Seismic attenuation in partially molten rock is poorly understood, due in part to the difficulty of making such measurements. Experimental values of $Q_{p}$ for partially molten basalt range from 6 [Sato et al., 1988, 1989] to 10 [Murase and McBirney, 1973; Sato and Manghnani, 1985; Manghnani et al., 1986], but these measurements were made at high frequencies $(>100 \mathrm{kHz})$ and may overpredict attenuation of lower-frequency $(10 \mathrm{~Hz})$ seismic energy. Wilcock et al. [1992] estimate a $Q_{p}$ of 25-60 and a markedly higher shear wave attenuation (i.e., lower $Q_{s}$ ) for the lower crust below the AMC. We have chosen a value of 20 for $Q_{p}$ within the magma sill, a value intermediate to that predicted for partial and pure melt, and a value of 10 for $Q_{s}$. For thin sills our results are not especially sensitive to the assumed $Q$ values since the amount of time that seismic energy spends within the sill is quite small.

\subsubsection{Evidence for a Thin Magma Sill: Waveform Modeling of the Near-Offset Trace}

Results from waveform modeling the near-offset trace at three of the study areas are displayed in Figure 4-7. The composite reflection term $R_{c}$ was calculated by the 

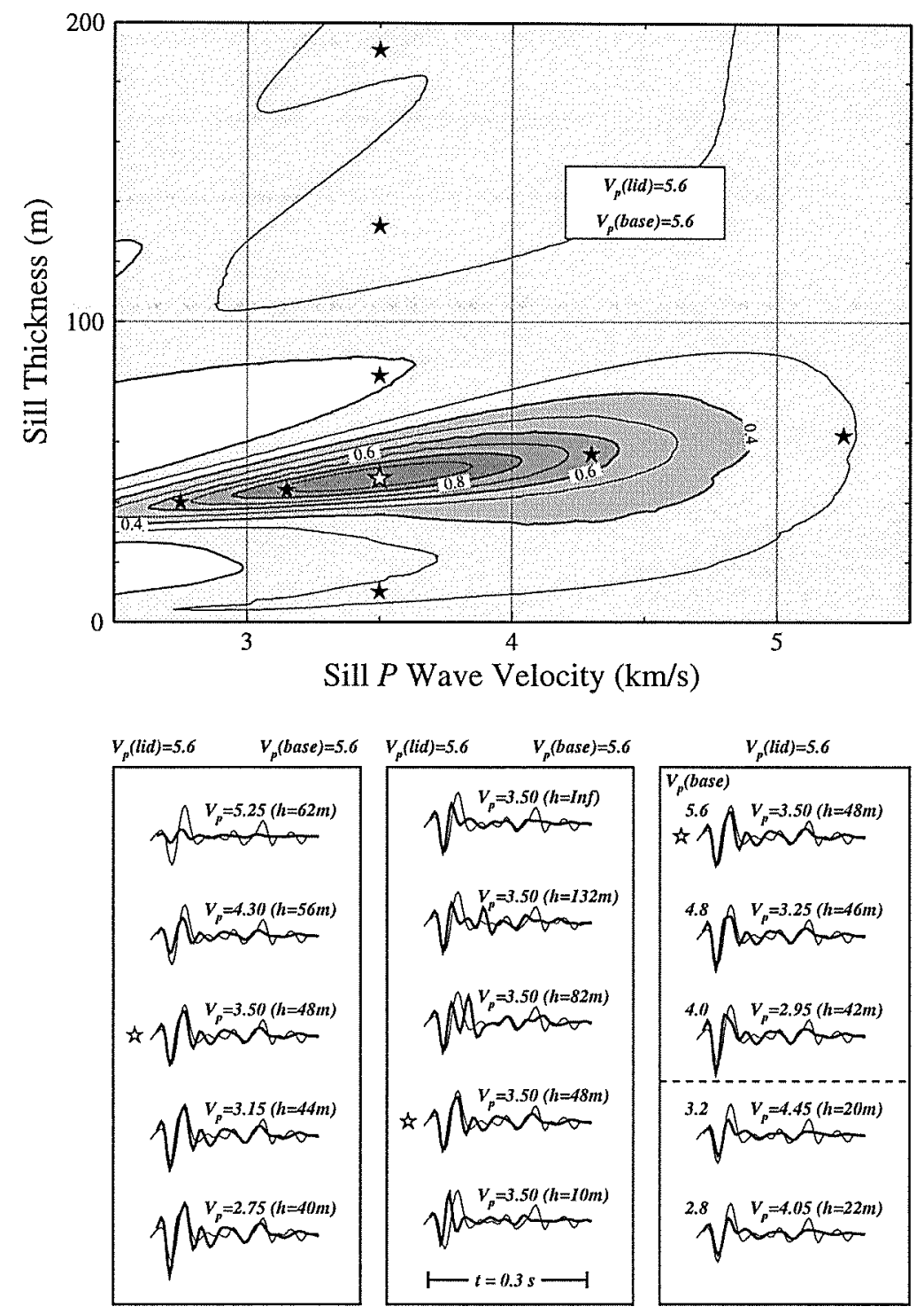

Figure 4-7a: Waveform modeling results for SEPR03-19 $36^{\prime} \mathrm{S}$. (top) The quality of fit between modeled AMC waveforms and the observed data is contoured for a lid and basal velocity of $5.6 \mathrm{~km} / \mathrm{s}$ as a function of sill thickness and $V_{p}$. The fitting parameter used for comparison is the sum of the squared residuals, inverted and normalized to ' 1 ' so that the quality of fit between the two waveforms is measured by its proximity to unity. (bottom) Model waveforms (thick curves) corresponding to the thickness and $V_{p}$ values marked by stars in the top panel plotted against the near-offset AMC reflection in the data (thin curves). (left) Effect on the waveform of moving along the best fitting constant-delay ridge in thickness- $V_{p}$ space. (middle) Effect on the waveform of varying only the sill thickness. (right) Best fitting waveforms at varying basal velocities. Open stars mark the best model unless a lower basal velocity (black arrow) better fits the data. A horizontal dashed line marks the boundary between waveforms from a mush sill and those from a double transition. At all study locations a mush sill fits the data better than a double transition. 

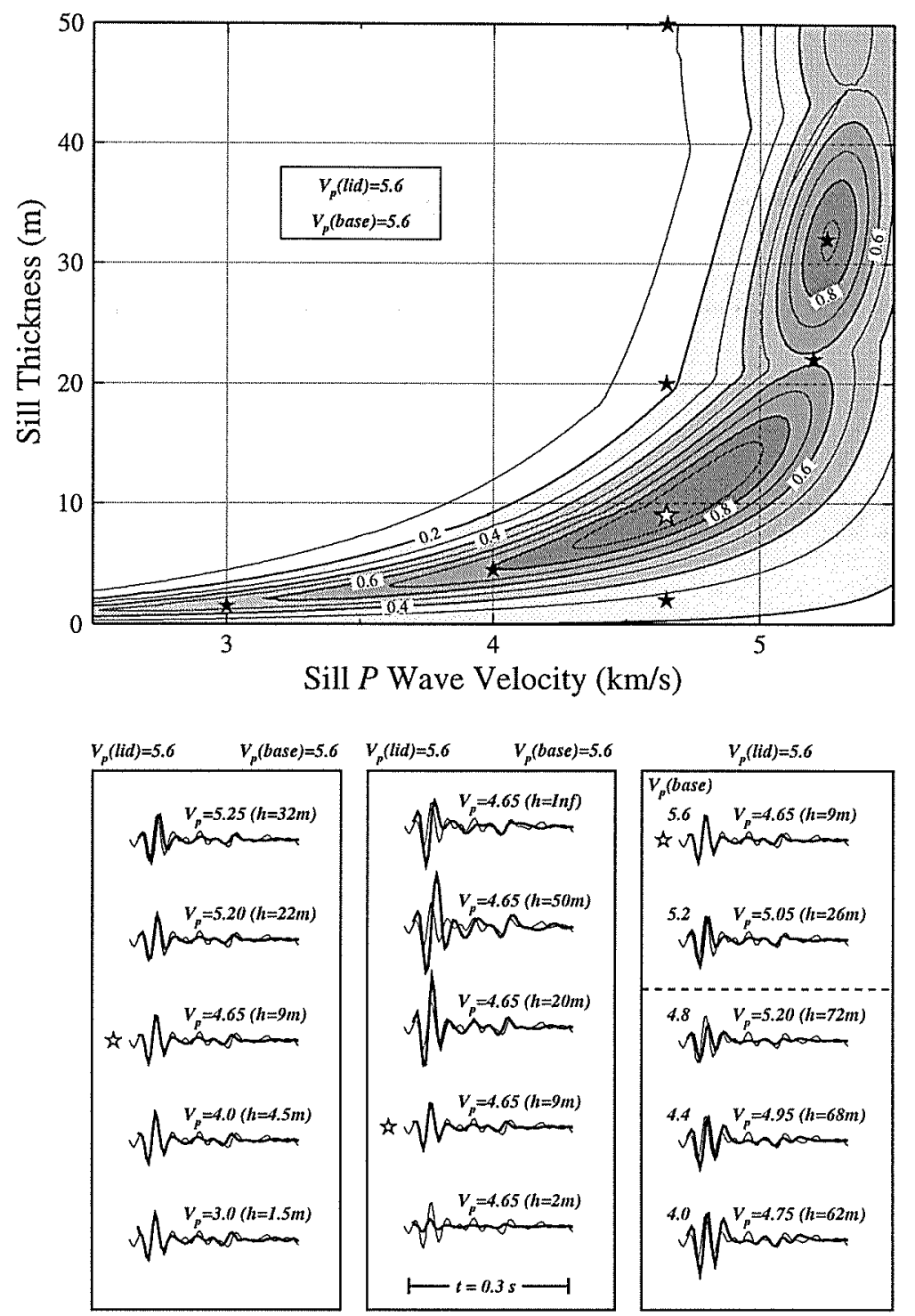

Figure 4-7b: Same as Figure 4-7a, except for SEPR09-17 $33^{\prime} \mathrm{S}$.

method of Meissner and Meixner [1969] and Juhlin and Young [1993] for the slowness value corresponding to the nearest-offset trace $(p=0.01 \mathrm{~s} / \mathrm{km})$, thereby simplifying Equation 4.3. Shear wave velocities were treated as a function of $V_{p}$ and were retained in the model solely for their effect on the $P$ wave reflection coefficient (little shear wave conversion occurs at near-normal incidence, so waveform amplitude is largely independent of sill $S$ wave velocity). The observed waveform amplitudes were normalized by their calculated near-normal incidence reflection coefficient $\left(R_{\mathrm{AMC}}\right)$ before 

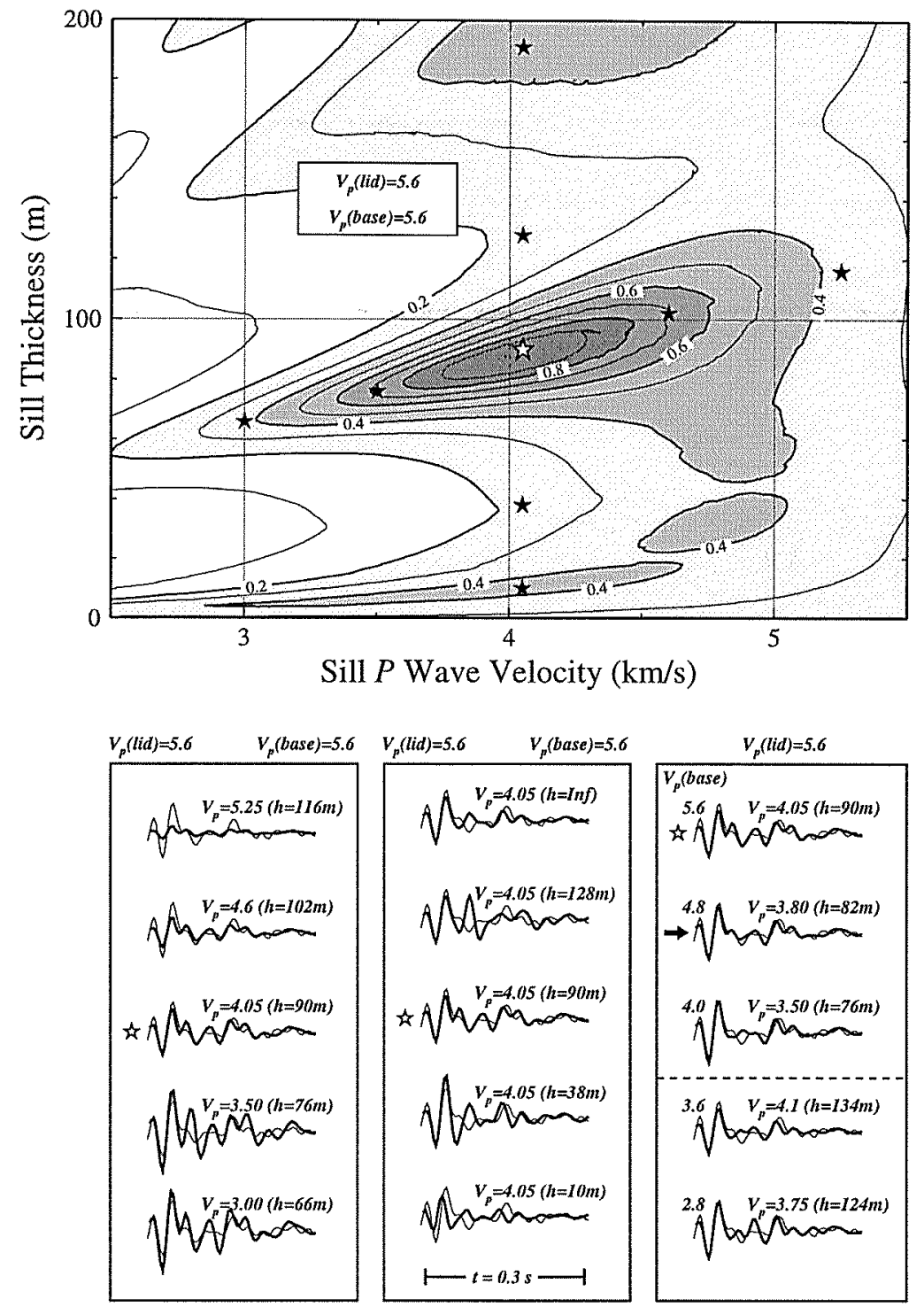

Figure 4-7c: Same as Figure 4-7a, except for NEPR41-9 $39^{\prime} \mathrm{N}$.

being compared with the model waveforms. The addition of amplitude information into the waveform modeling not only provides better constraints on $P$ wave velocities, but it also allows a more accurate estimate of the thickness of very thin magma sills (see bottom middle panel of Figure 4-7b) [Widess, 1973; Kallweit and Wood, 1982].

None of the waveforms calculated assuming a magma half-space beneath the AMC reflector match the observed data (Figure 4-7). Either the observed waveform is phase-shifted with respect to the source (Figures $4-7 \mathrm{a}$ and $4-7 \mathrm{~b}$ ), or it exhibits a sec- 
ondary pulse arriving later than the primary reflection (Figure 4-7c). The anomalous phase of the AMC event can not be modeled by the reflection from a simple interface nor by a steep gradient zone within the molten body. Only the interference between reflections from two closely spaced interfaces can explain the observed waveforms. In all study locations the observed waveforms are best modeled by reflections from the top and bottom of a magma sill, rather than a double transition to partial melt (bottom right panels of Figure 4-7).

If a normal-polarity basal reflection interferes with the phase-reversed roof reflection, the composite waveform will be phase-shifted by a variable amount depending on the sill thickness and velocities within the sill. This effect can be seen in the observed waveforms shown in Figures 4-7a and 4-7b and is well illustrated by comparing the model curves shown in Figure 4-7b for sill thicknesses of 9, 20, and $50 \mathrm{~m}$ with the predicted waveform for no basal reflector. Variations in sill thickness also strongly affect waveform amplitudes. At sill thicknesses less than one-quarter of the dominant wavelength $(30-40 \mathrm{~m})$ the basal reflection no longer phase shifts the waveform but begins to destructively interfere with the roof reflection, thereby reducing its total amplitude [Kallweit and Wood, 1982]. This effect can be seen in the model waveforms shown in Figure 4-7b as sill thickness is reduced from 20 to $2 \mathrm{~m}$. At thicknesses greater than one-half the seismic wavelength the delay of the basal reflection is enough to separate it from the roof reflection. In this case the initial pulse is phase-reversed, and a second, positive-polarity pulse appears behind the roof reflection. This pattern can be seen in the observed data from $9^{\circ} 39^{\prime} \mathrm{N}$ (Figure 4-7c) but does not match the other data sets well (Figures 4-7a and 4-7b). The anomalous phase and complicated waveform of the AMC reflection thus provide strong evidence for the existence of a thin magma sill at the top of the AMC.

The linear features trending toward larger thicknesses and $P$ wave velocities in the fitting parameter surfaces of Figure 4-7 delineate ridges of constant waveform offset. Any change in the waveform along these ridges can only be due to variations 
in amplitude. The primary control on amplitude is $V_{p}$. It determines the amount of total energy reflecting from the melt lens and the amplitude ratio between the roof and the basal reflection. The effect of variations in $P$ wave velocity on the waveform is shown in the bottom left panels of Figure 4-7.

We have used the dependence of waveform amplitude and phase on sill thickness and $P$ wave velocity to determine the combination of properties that best fit the observed data. Estimated sill thicknesses range from 9 to $82 \mathrm{~m}$ and are consistent with previous results at the EPR [Harding et al., 1989; Kent et al., 1993; Detrick et al., 1993]. Sill $P$ wave velocities vary between 3.5 and $4.65 \mathrm{~km} / \mathrm{s}$. The thickest sill is at $9^{\circ} 39^{\prime} \mathrm{N}(82 \mathrm{~m})$, while the thinnest is at $17^{\circ} 33^{\prime} \mathrm{S}(9$ or $32 \mathrm{~m})$ where there is evidence of recent volcanic activity. At the four sites along the southern EPR, lower $P$ wave velocities are associated with thicker sills.

\subsubsection{Evidence for Nonzero Shear Wave Velocities: Ampli- tude Versus Slowness Analysis}

The AVS behavior of reflections from a magma sill depends on sill thickness and compressional and shear wave velocities in different ways. The effects of variations in each of these parameters are shown in Figure 4-8. Model seismograms for AVS analysis are generated by the enhanced reflectivity method of Kennett $[1980,1983]$ in a fashion similar to Equation 4.3. At near-normal incidence the reflection amplitude depends primarily on compressional wave velocity and, because of the basal reflector, on sill thickness (Figures 4-8a and 4-8b). Little conversion to shear motion occurs at these slownesses, making the amplitude largely independent of $S$ wave velocity (Figure 4-8c). Sill thickness influences the reflection amplitude by a complicated pattern of constructive and destructive tuning of the basal reflection to the roof reflection, with vastly different thicknesses producing similar AVS patterns (Figure 4-8a). In contrast, the effect of $P$ wave velocity variations on the AVS curve follows a simple pattern (Figure 4-8b). Decreasing the $P$ wave velocity in the sill not only increases reflection 
a) $\mathrm{V}_{\mathrm{s}}=1.5 \mathrm{~km} / \mathrm{s} \& \mathrm{~V}_{\mathrm{p}}=3.75 \mathrm{~km} / \mathrm{s}$

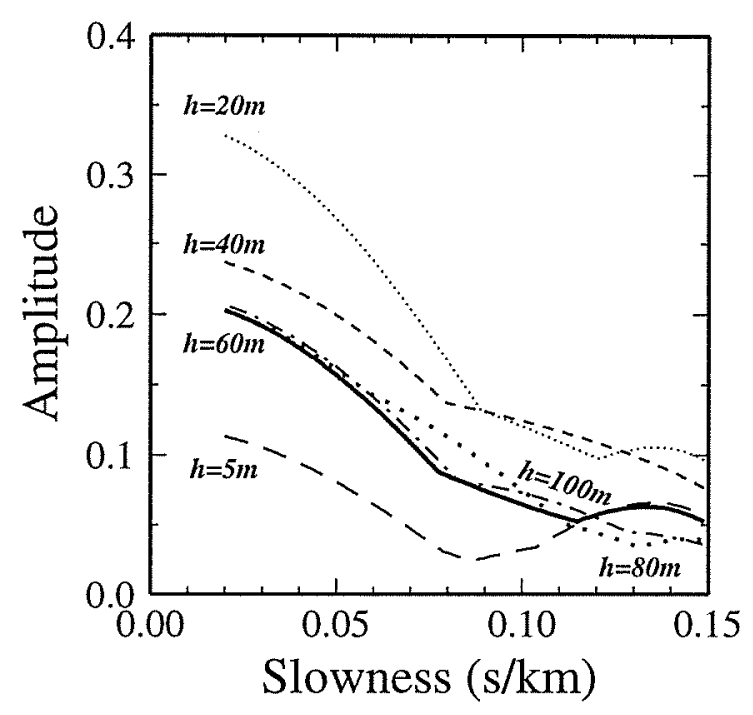

b) $\quad \mathrm{V}_{\mathrm{s}}=1.5 \mathrm{~km} / \mathrm{s} \& \mathrm{~h}=60 \mathrm{~m}$

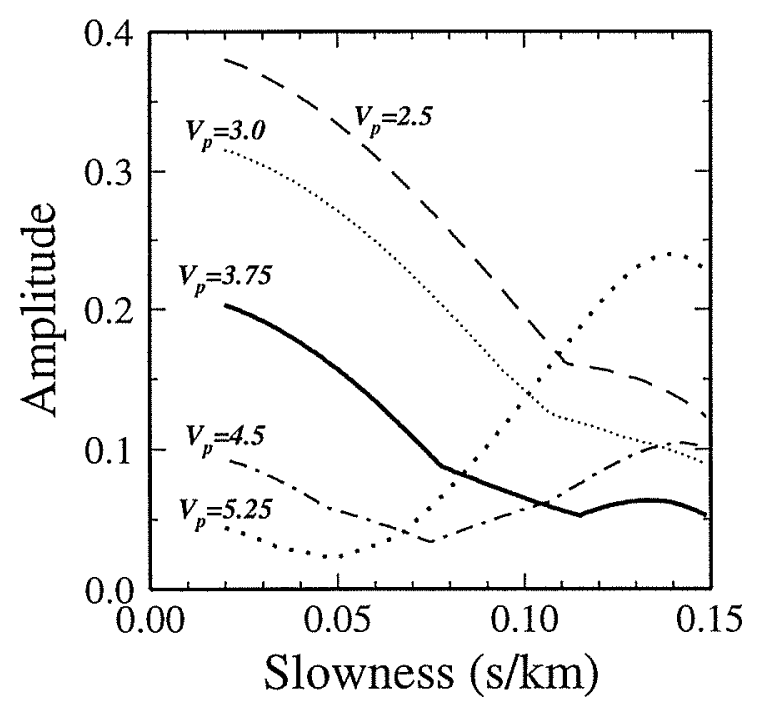

c) $\quad \mathrm{V}_{\mathrm{p}}=3.75 \mathrm{~km} / \mathrm{s} \& \mathrm{~h}=60 \mathrm{~m}$

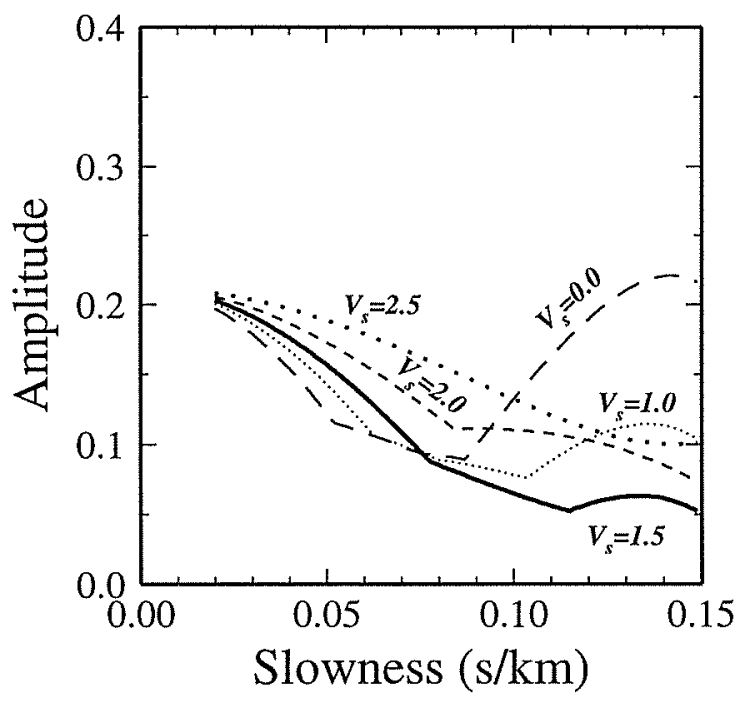

Figure 4-8: Effect of (a) melt lens thickness, (b) $V_{p}$, and (c) $V_{s}$ variations on AMC amplitude as a function of slowness. The solid curve in all three panels is a reference model, the parameters of which best fit the SEPR35-16 $52^{\prime} \mathrm{S}$ AVS curve $\left(V_{p}=3.75 \mathrm{~km} / \mathrm{s}, V_{s}=1.5\right.$ $\mathrm{km} / \mathrm{s}$, and $h=60 \mathrm{~m}$, with host rock properties of $V_{p}=5.6 \mathrm{~km} / \mathrm{s}$ and $V_{s}=3.23 \mathrm{~km} / \mathrm{s}$ ). 
amplitudes by increasing the impedence contrast across the roof of the sill but also shifts to greater slownesses the point where the reflection event reverses phase and becomes normal polarity (see Figures $4-2 \mathrm{c}$ and $4-2 \mathrm{~d}$ ). If the sill $P$ wave velocities are low enough (i.e., if the impedence contrast is high enough), there will be no phase inversion in the AMC reflection at large slowness values.

The shape of the AVS curve is particularly sensitive to the shear wave velocity contrast across the rock/magma interface, since it governs the amount of shear wave conversion. As sill $S$ wave velocity decreases, a proportionally greater amount of reflected energy is converted to shear motion, thereby producing a faster initial falloff in reflection amplitude with increasing slowness (Figure 4-8c). If the $S$ wave velocity is zero within the sill, amplitudes decrease rapidly with increasing slowness but display a diagnostic increase at larger slownesses (Figure 4-8c). The shape of the AVS curve is thus more sensitive to variations in sill $P$ and $S$ wave velocities, whereas AVS analysis provides comparatively weak constraints on sill thickness (Figure 4-8a). We therefore assume that the sill thicknesses inferred from waveform modeling of the near-offset traces are correct and model the observed AVS curves by varying only $V_{p}$ and $V_{s}$.

Figure 4-9 contours the quality of fit in $V_{p}-V_{s}$ space that each model produces with the data and displays the AVS pattern for the best fitting model at the same study locations as in Figure 4-7. None of the observed AVS curves show the diagnostic increase in amplitude at large slownesses expected if the magma sill were purely molten (i.e., if $V_{s}=0 \mathrm{~km} / \mathrm{s}$ ). The choice of lid velocity and crustal attenuation structure does not affect this result, since they do not control the overall shape of the AVS curves. The lowest shear wave velocity $(1.15 \mathrm{~km} / \mathrm{s})$ is observed at $19^{\circ} 36^{\prime} \mathrm{S}$, where the reflection amplitude falls off rapidly to values of less than one-tenth the initial values (Figure 4-9a). The highest shear wave velocity $(2.3 \mathrm{~km} / \mathrm{s})$ is observed at $17^{\circ} 33^{\prime} \mathrm{S}$, where reflection amplitudes remain low at all slownesses (Figure 4-9b). The other three locations, including $9^{\circ} 39^{\prime} \mathrm{N}$, exhibit AVS curves and shear wave velocities intermediate between these two extremes (Figures 4-4c, 4-4d, 4-4e and 4-9c). 

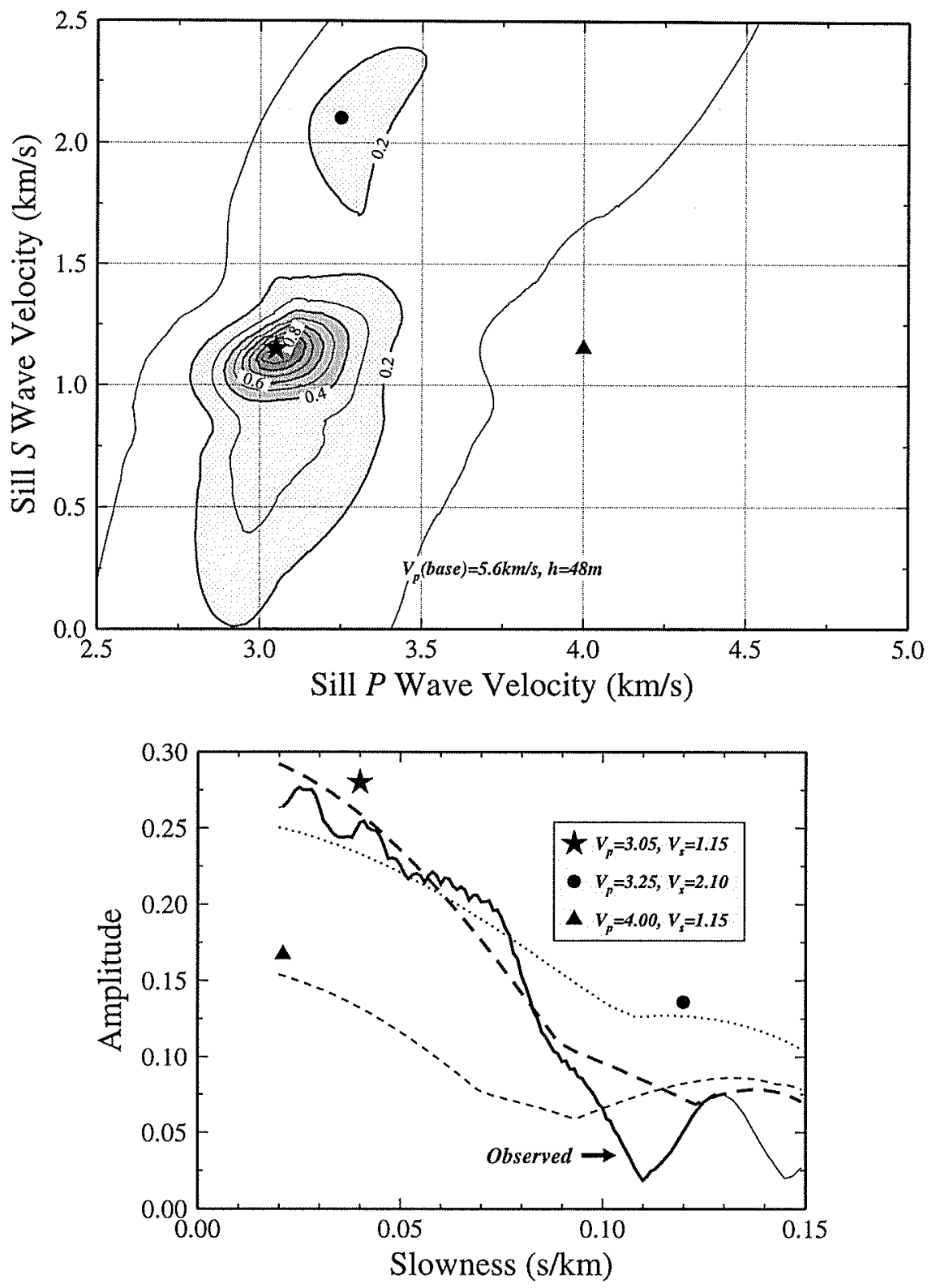

Figure 4-9a: AVS modeling results for SEPR03-19³6'S. Best fitting thickness in each data set's corresponding waveform study was held constant in AVS modeling. (top) Contoured fitting parameter is the sum of the squared residuals, inverted and normalized to ' 1 '. A star marks the best fitting model, and triangles and bullets mark additional models in $V_{s}-V_{p}$ space, the corresponding AVS curves of which (bottom) are compared to that of the data. Those parts of the data AVS curves which are not modeled are plotted with a finer curve thickness. 

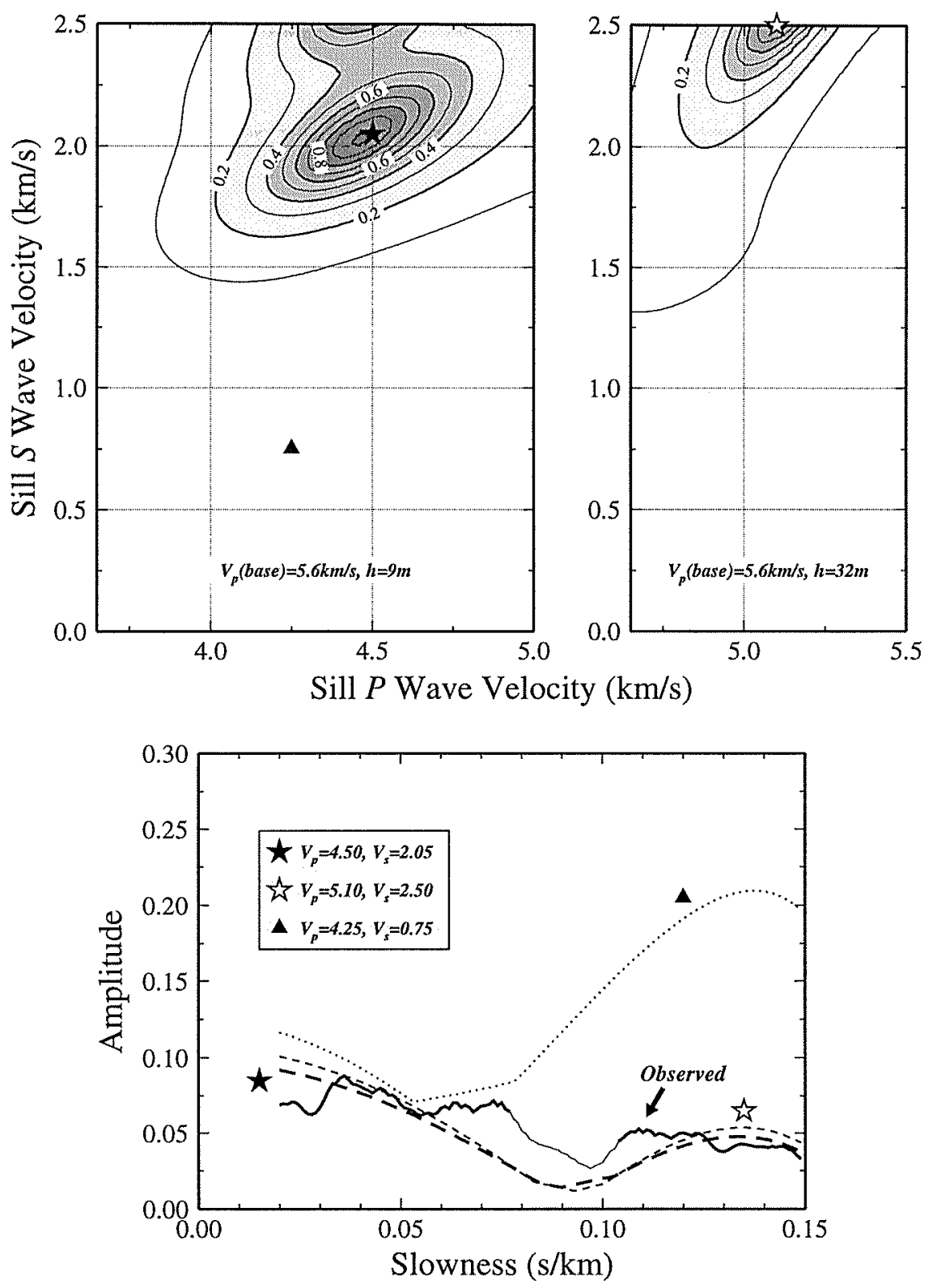

Figure 4-9b: Same as Figure 4-9a, except for SEPR09-17 33’S. Two thicknesses are examined because waveform modeling was not able to unambiguously determine a single thickness for this location. 

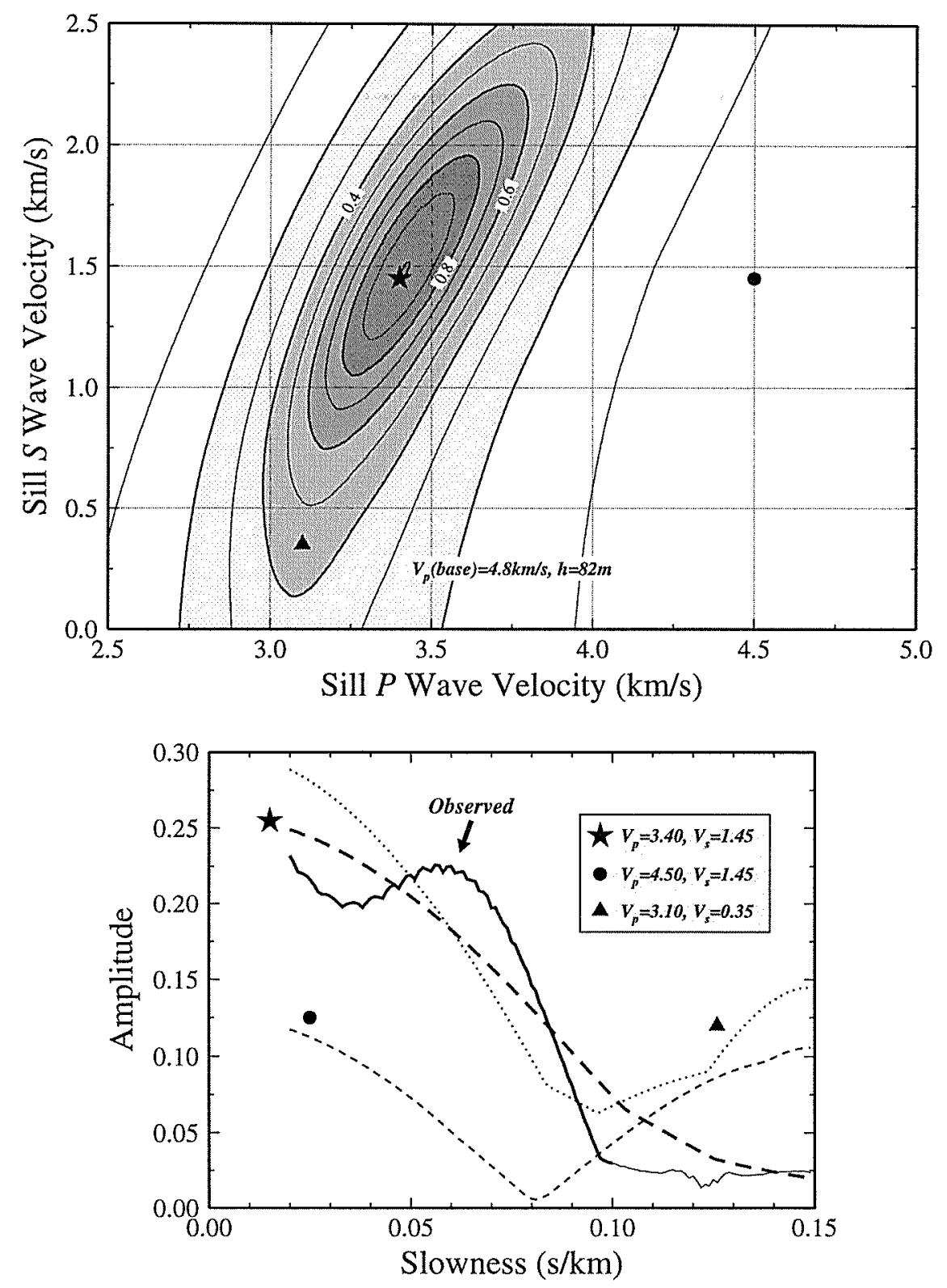

Figure 4-9c: Same as Figure 4-9a, except for NEPR41-9 $39^{\circ} \mathrm{N}$. 


\subsection{Discussion}

We have shown that $\mathrm{AMC}$ reflections from the EPR are well modeled by a composite waveform derived from the interference between reflections from the roof and base of a magma sill no thicker than $\sim 100 \mathrm{~m}$. The sill is characterized by compressional wave velocities between 3.5 and $4.65 \mathrm{~km} / \mathrm{s}$ and nonzero shear wave velocities between 1.15 and $2.3 \mathrm{~km} / \mathrm{s}$. Along the southern EPR we find that the thickest magma sills are associated with the lowest $P$ and $S$ wave velocities (Table 4.1).

The comparatively high $P$ wave velocities and nonzero shear wave velocities inferred from the amplitude and phase of the AMC reflection suggest that the magma sill found at the roof of the AMC is not entirely molten. The effect of melt on seismic properties is discussed in detail by Schmeling [1985] and Forsyth [1992]. In Figure 4-10 we compare our measurements of $V_{p}$ and $V_{s}$ in the magma sill with experimental results [after Murase and McBirney, 1973] for basaltic magmas. Laboratory results suggest that at temperatures above the basalt solidus, $V_{p}$ drops precipitously from $\sim 5.5 \mathrm{~km} / \mathrm{s}$ to values near $2.5 \mathrm{~km} / \mathrm{s}$. $S$ wave velocities remain near $2.5 \mathrm{~km} / \mathrm{s}$, then drop rapidly to zero once the $P$ wave velocity has begun leveling off. Initiation of the $P$ wave velocity drop is associated with a melt content near $5 \%$ [Sato et al., 1989; Forsyth, 1992] at which point melt becomes connected across grain boundaries. The shear wave falloff corresponds to a higher melt content $(\sim 20-30 \%)$ at which the crystals no longer form an aggregate that is able to support shear stress. One can infer from these results that the magma sill at $19^{\circ} 36^{\prime} \mathrm{S}$ contains $>20 \%$ melt, whereas the one at $17^{\circ} 33^{\prime} \mathrm{S}$ may contain less than this amount. These experimental values must be interpreted with caution since they were not obtained from gabbroic compositions thought to be representative of the magma sill, nor were they measured under confining pressures appropriate to midcrustal depths using seismic frequencies. However, there is little dispute that a purely molten sill should be characterized by a low $P$ wave velocity $(\sim 2.5 \mathrm{~km} / \mathrm{s})$ and a zero shear wave velocity. The higher $P$ and $S$ wave velocities inferred for the magma sill at the EPR indicate that it consists of a partially 


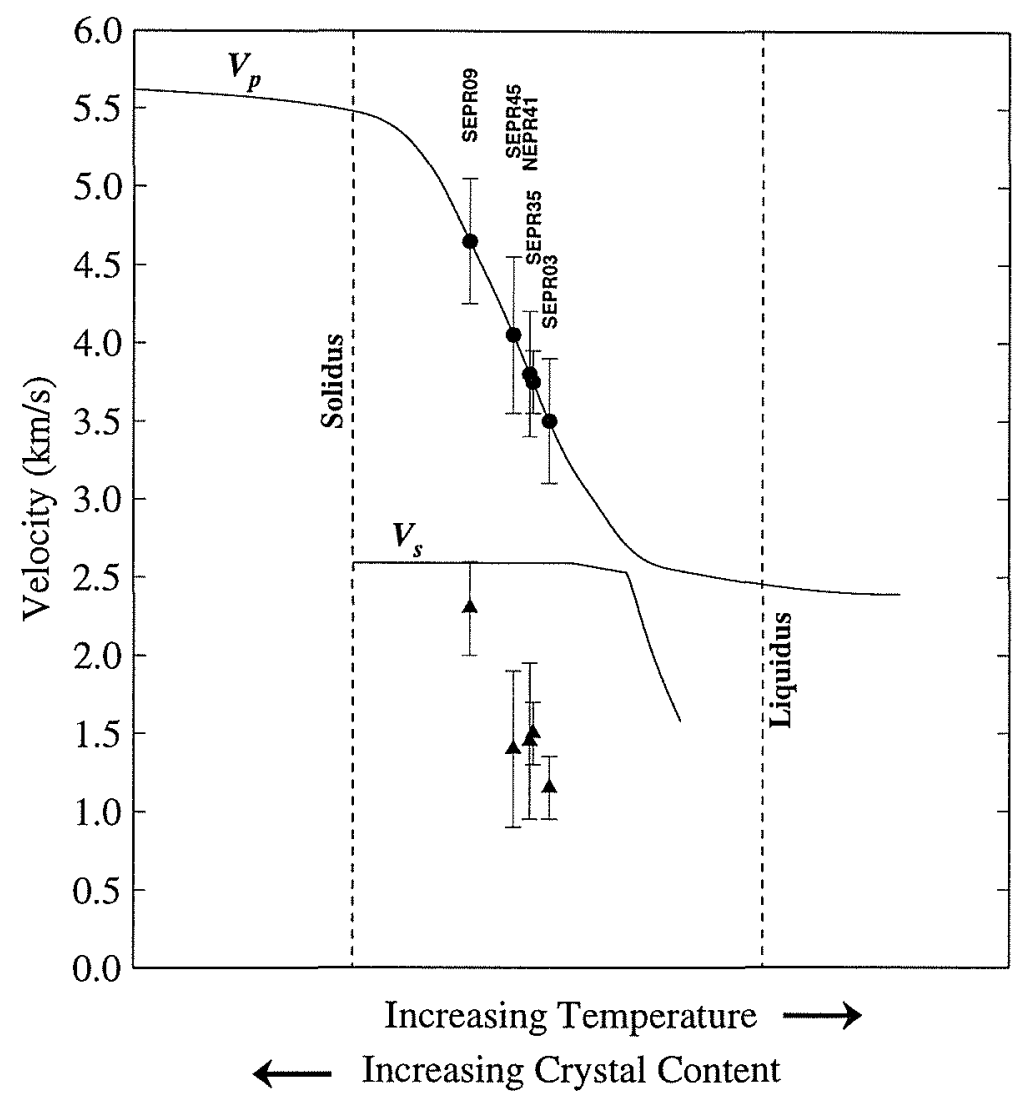

Figure 4-10: Variation of seismic velocity with temperature and/or crystal content of a basaltic composition. Solid curves show experimentally derived values [after Murase and McBirney, 1973]. $P$ wave and $S$ wave velocities predicted in this study are plotted according to the experimental $P$ wave velocity curve by dots and triangles, respectively.

molten crystal mush. The term "melt lens" often used to describe this feature is thus somewhat of a misnomer. The thicker magma sills (e.g., at $19^{\circ} 36^{\prime} \mathrm{S}$ and $9^{\circ} 39^{\prime} \mathrm{N}$ ) appear to be largely molten, while the comparatively high $P$ and $S$ wave velocities associated with thinner sills (e.g., $17^{\circ} 33^{\prime} \mathrm{S}$ ) indicate a relatively high crystal content.

A largely crystalline magma sill is in contrast to lavas erupted along the EPR, which typically contain less than $10 \%$ crystals in suspension [Sinton and Detrick, 1992]. It is possible that the source of the erupted basalts is a deeper magma reservoir (e.g., the base of the crust). In this case, the crystal content of the sill could be greatly reduced immediately prior to dike injection and eruption by the sudden introduction of melt from this deeper source. The magma sill would then primarily reflect the 
cooling residue of the last eruptive event, thereby playing a relatively passive role in the emplacement process. However, it may be possible to erupt largely aphyric lavas from a crystal-rich magma sill without invoking a sudden injection of melt from a deeper reservoir. One such mechanism is that the feeder dike taps a crystal-free layer within the magma sill. The volume of a typical eruption along the EPR has been estimated from submersible evidence to be $5 \times 10^{5} \mathrm{~m}^{3}$ per kilometer of ridge [Gregg et al., 1996]. Given a typical sill width of $750 \mathrm{~m}$ and a dike height and width of $1.2 \mathrm{~km}$ and $1 \mathrm{~m}$, respectively, this intralens melt layer need be only about $2 \mathrm{~m}$ thick to be the source of such a flow. Such a thin melt layer would not be seismically resolvable. Even if the feeder dike taps a uniformly crystal-rich sill, melt may be preferentially squeezed out through the interconnected pore space. Calculations of magma sill compaction assuming simple Poiseuille flow for the high-viscosity crystal matrix and Darcy flow through a porous medium for the interstitial melt show that the melt flux will indeed be much greater than the matrix (crystal) flux, since the compaction length of the crystal matrix (hundreds to thousands of meters [Spiegelman, 1993]) is much greater than either the sill thickness or dike width (J. M. Pringle, personal communication, 1996). These calculations also suggest that without a significant lateral pressure gradient, the high crystal content of the sill will severely inhibit mixing of magma along the rise axis. Although the assumptions in these compaction models break down if eruption volumes approach that of the magma sill or at melt contents that exceed the rigidus [Marsh, 1989; Sinton and Detrick, 1992], they are applicable to typical flow volumes observed at the EPR and magma sills exhibiting a nonzero shear wave velocity.

In Figure 4-11 we compare our determinations of magma sill thickness and velocity with variations in rise crest depth, axial volume, and AMC depth along the southern EPR. Along-axis variations in ridge crest morphology (e.g., depth and axial volume) have been widely interpreted as reflecting along-axis variations in magma supply [Macdonald et al., 1984, 1988; Scheirer and Macdonald, 1993]. Shallow, inflated sec- 

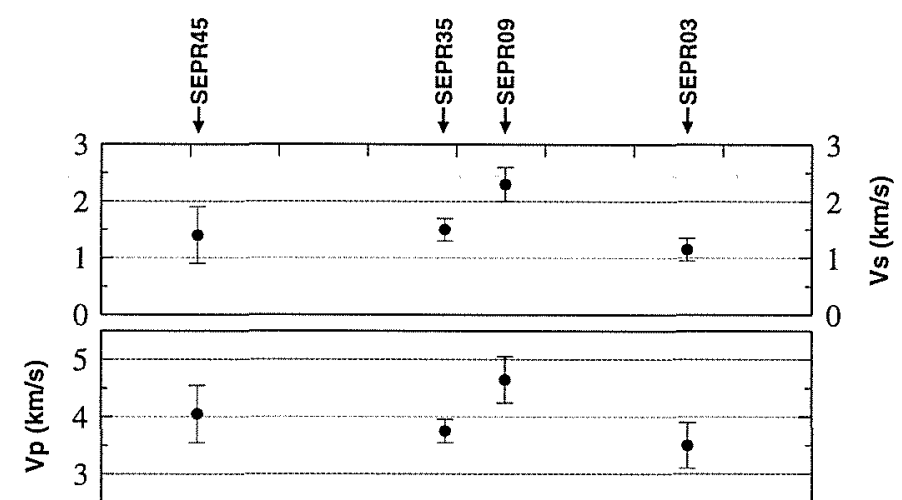

$\begin{array}{rr}100 & \bar{E} \\ 75 & \frac{5}{0} \\ 50 & \frac{8}{3} \\ 25 & \frac{5}{0} \\ 0 & \text { F }\end{array}$
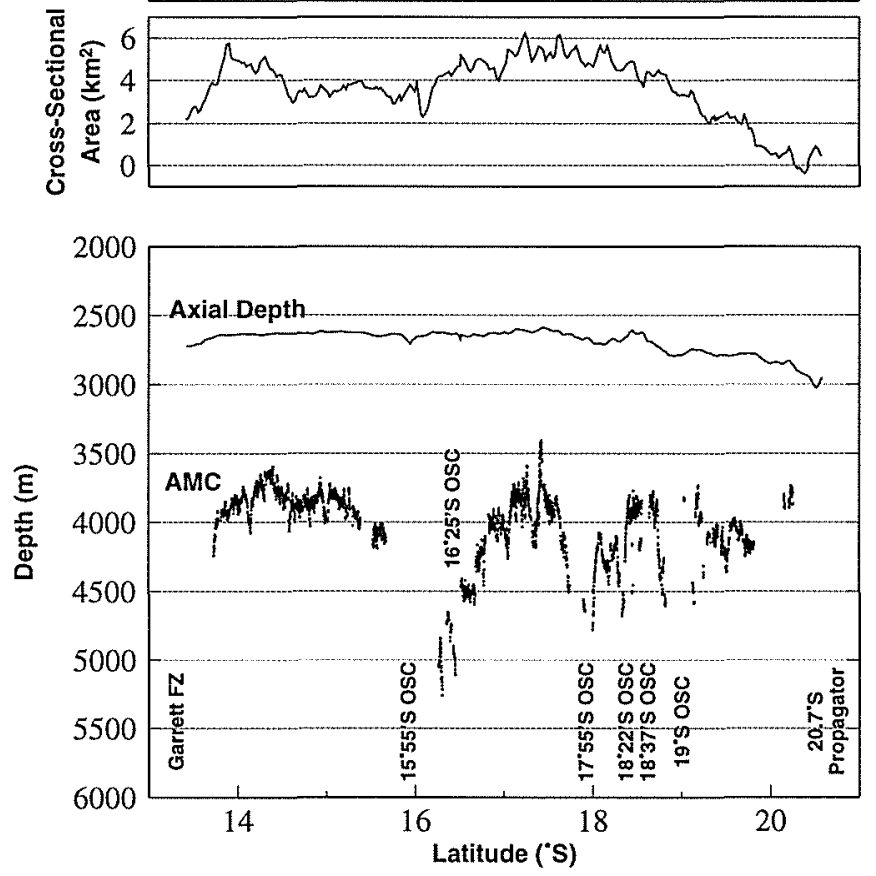

Figure 4-11: Sill thickness, $V_{p}$, and $V_{s}$ for the four study locations along the southern EPR. These values are plotted against along-axis variations in axial volume [after Scheirer and Macdonald, 1993], axial depth, and AMC depth below the seafloor (E.E. Hooft, personal communication, 1996). 
tions of ridge, like those found near $17^{\circ} \mathrm{S}$ and $14^{\circ} \mathrm{S}$, are believed to be magmatically robust, while deeper, narrower sections of ridge (e.g., 19-20'S) are interpreted as having a lower magma budget. One might therefore expect that the magma sill beneath shallow, inflated sections of the rise axis would be thicker and more molten than elsewhere along the ridge. There does not, however, appear to be a simple relationship between morphologic indicators of magma supply and magma sill properties. Of the five sites studied the thickest and most molten sill is located at $19^{\circ} 36^{\prime} \mathrm{S}$, where the rise axis is deep and narrow, while the thinnest and most crystalline sill is located at $17^{\circ} 33^{\prime} \mathrm{S}$, where the rise axis is very broad and shallow (Figure $4-11$ ). Similarly, there is little correlation between the depth of the magma sill below the seafloor and its thickness or crystallinity, as might be expected if it forms at a depth of neutral buoyancy $\left[\right.$ Ryan, 1987, 1993]. The magma sills at $19^{\circ} 36^{\prime} \mathrm{S}$ and $17^{\circ} 33^{\prime} \mathrm{S}$ have very different properties but lie at nearly the same depth below the seafloor. Also, the thick, largely molten sill at $9^{\circ} 39^{\prime} \mathrm{N}$ lies nearly $500 \mathrm{~m}$ deeper than the thinner, more crystalline magma sills found along parts of the southern EPR.

The lack of correlation between magma sill properties, like thickness and crystallinity, and morphologic indicators of magma supply, may reflect different timescales of variability. Axial morphology presumably reflects a long-term (approximately $>10^{3}-10^{4}$ years) thermal balance between heat supplied by intrusion of new magma and hydrothermal cooling. A higher magma supply results in hotter, less dense lower crust and upper mantle and a thicker crust, leading to the formation of a shallower, broader ridge [Scheirer and Macdonald, 1993] and a shallower AMC [Hooft and Detrick, 1993]. However, the properties of the magma sill may be much more closely tied to the eruptive cycle, which at these spreading rates may occur on timescales of only $10-10^{2}$ years. Replenishment of the AMC by a new batch of magma may lead to the ponding of more melt at the roof of the $\mathrm{AMC}$, resulting in a thicker, more molten sill. Dike intrusion and eruption will drain off the melt-rich portion of the sill, leaving behind a thin, largely crystalline residue. If this interpretation is correct, 
then the thin, largely crystalline magma sill at $17^{\circ} 33^{\prime} \mathrm{S}$ would represent a residual magma reservoir following the recent eruption of this section of ridge [Mutter et al., 1995], while the thicker, more molten sill at $19^{\circ} 36^{\prime} \mathrm{S}$ may reflect a replenished AMC,

possibly indicating an impending eruption. It may be more than coincidental that a thick, largely molten magma sill was imaged at $9^{\circ} 39^{\prime} \mathrm{N}$ in 1985 and that the section of ridge immediately to the north of this area subsequently erupted in 1991 [Haymon et al., 1993].

\subsection{Conclusions}

We have analyzed the AMC event observed in single-ship, multichannel reflection data at selected locations along the EPR in order to constrain the physical properties of the magma body thought to cause this reflection. Although information from wide-aperture seismic studies, such as two-ship wide-aperture profiles (WAPs) and expanding spread profiles (ESPs), would increase the accuracy of these results by, for example, better constraining the velocity immediately above the magma sill, these single-ship observations contain enough information on the midcrustal to uppercrustal structure of the rise crest to provide geologically significant constraints on midcrustal magma bodies at the EPR. Four locations along the EPR south of the Garrett fracture zone and one at $9^{\circ} 39^{\prime} \mathrm{N}$ were studied. The following conclusions are based on both waveform modeling and AVS analysis results at these locations:

1. Waveform modeling of the near-offset AMC reflection requires the existence of a second reflection with a positive impedence contrast below the initial roof reflection (i.e., the existence of a magma sill clearly defined at its roof and base) at the top of the AMC. Neither a melt half-space nor a double transition matches the data well.

2. The magma sill has a thickness of the order of 10-100 m. Greater thicknesses would result in a distinct basal reflection behind the initial AMC event; a feature that is not observed in the data. 
3. High $P$ wave velocities and nonzero shear wave velocities indicate that the magma sill is not entirely molten. In general, thicker sills are observed to be more molten than thinner sills, a pattern that may reflect the stage of that section of rise axis within the volcanic cycle of eruption and replenishment.

4. No simple relationship exists between magma sill properties (i.e., thickness, $V_{p}$, $V_{s}$ ) and $\mathrm{AMC}$ depth or axial morphology. We argue, however, that AMC variations act on a spatially short and temporally rapid timescale and do not necessarily correlate with the longer-term magma budget of the rise axis.

\subsection{Acknowledgments}

We wish to thank Paul Henkart and the Scripps Industrial Associates for use of the SIOSEIS processing package. This manuscript was greatly improved by the helpful comments of J. M. Sinton and an anonymous reviewer. We would also like to thank D. J. Fornari, G. Hirth, T. K. P. Gregg, D. Lizarralde, P. B. Kelemen, M. K. McNutt, J. M. Pringle, D. S. Scheirer, H. Schouten, and M. A. Tivey for their insightful comments and E. E. Hooft for her discussions and permission to include her data. This study was funded by NSF grant OCE-9402033 to R. S. Detrick and an ONR fellowship to S. A. Hussenoeder. Woods Hole Oceanographic Institution contribution 9117.

Hussenoeder, S. A., J. A. Collins, G. M. Kent, R. S. Detrick, and the TERA Group, Seismic analysis of the axial magma chamber reflector along the southern East Pacific Rise from conventional reflection profiling, J. Geophys. Res., 101, 22,087-22,105, 1996. Copyright by the American Geophysical Union. Reprinted with permission. 


\section{Chapter 5}

\section{Fine-scale seismic structure of young upper oceanic crust at $17^{\circ} 20^{\prime} \mathrm{S}$ on the fast spreading East Pacific Rise}

\subsection{Introduction}

Over two decades ago, seismic refraction experiments began providing strong evidence to support the concept of a layered upper ocean crust [Houtz, 1976; Houtz and Ewing, 1976]. Since that time, numerous seismic experiments along the fast-spreading East Pacific Rise (EPR) have combined to make it the most extensively studied ridge in the world. They include refraction experiments (ROSE [Bratt and Purdy, 1984; Ewing and Purdy, 1982; Purdy, 1982; Fischer and Purdy, 1986], MAGMA [McClain et al., 1985], EXCO [Grevemeyer and Weigel, 1997; Grevemeyer et al., 1997] and tomographic studies [Burnett et al., 1989; Toomey et al., 1990; Caress et al., 1992; Toomey et al., 1994]), single-ship multichannel seismics (MCS) [Detrick et al., 1993; Harding et al., 1993; Kent et al., 1994; Carbotte et al., 1997; Hooft et al., 1997a], two-ship wide aperture profiles (WAP) [Vera and Diebold, 1994; Kappus et al., 1995], two-ship expanding spread profiles (ESP) [Harding et al., 1989; Vera et al., 1990], and, more recently, on-bottom seismics [Christeson et al., 1992, 1996]. In combination, 
these methods have been a powerful tool in our effort to understand the structure of upper oceanic crust.

Since the majority of seismic experiments have focused on the EPR, the standard model of upper crustal structure is biased toward fast spreading centers. This model consists of two layers: a low-velocity layer $2 \mathrm{~A}(3-4 \mathrm{~km} / \mathrm{s})$, overlying a higher velocity $(5.2 \pm 0.4 \mathrm{~km} / \mathrm{s})$ layer 2B [Houtz, 1976; Houtz and Ewing, 1976]. The transition between these two seismic intervals is often a simple gradient [Harding et al., 1989; Kappus et al., 1995; Tolstoy et al., 1997], but can be more complicated [Vera et al., 1990; Detrick et al., 1993; Christeson et al., 1994a]. The thickness and internal structure of these two layers, and the transition between them, are a direct result of mid-ocean ridge processes, and have important implications for the emplacement and maturation of volcanically constructed crust.

The origin of the layer $2 \mathrm{~A} / 2 \mathrm{~B}$ transition is still a subject of debate. It has previously been interpreted as a porosity horizon within the extrusive section. The primary evidence that supports this interpretation is from Deep Sea Drilling Project/Ocean Drilling Program (DSDP/ODP) Hole 504B, where changes in seismic velocity and attenuation transition are associated with a reduction in bulk porosity of the extrusive section [Becker et al., 1989; Wilcock et al., 1992]. Alternatively, the layer 2A/2B boundary may represent the downward transition from extrusive basalts to sheeted dikes. This has become the preferred interpretation of most recent seismic studies [Herron, 1982; Toomey et al., 1990; Christeson et al., 1992, 1994a, 1996; Harding et al., 1993; Kent et al., 1994; Vera and Diebold, 1994; Kappus et al., 1995; Hooft et al., 1996], and is supported by submersible observations of exposed crustal sections [e.g., Francheteau et al., 1990], ophiolite studies [Nicolas, 1989], and models of extrusive emplacement [e.g., Cann, 1974]. However, a porosity boundary and lithological transition need not be mutually exclusive, and may actually be coincident near the ridge axis [Harding et al., 1993].

Seismic layer $2 \mathrm{~A}$ is observed to thicken two to threefold within $1-4 \mathrm{~km}$ of the 
rise axis [Harding et al., 1993; Kent et al., 1994; Carbotte et al., 1997], and then gradually thin with age [Houtz and Ewing, 1976; Becker et al., 1989]. This thickening profile is accompanied by an increase in basement velocity from $\sim 2.5 \mathrm{~km} / \mathrm{s}$ at the rise axis to $\sim 5 \mathrm{~km} / \mathrm{s}$ in crust 10 Myr old or greater. The initial off-axis thickening has previously been attributed to the downward propagation of fractures [McClain et al., 1985]. However, accumulating evidence suggests that layer $2 \mathrm{~A}$ is built up by successive eruptions both on-axis [Toomey et al., 1990; Vera and Diebold, 1994; Hooft et al., 1996] and off-axis [Macdonald et al., 1989], coupled with subsidence of the sheeted dikes [Cann, 1974; Kidd, 1977; Dewey and Kidd, 1977; Toomey et al., 1990; Christeson et al., 1996]. The long-term thinning of layer $2 \mathrm{~A}$ has often been attributed to a decrease in upper crustal porosity [Hyndman and Drury, 1976; Schreiber and Fox, 1976; Fox and Stroup, 1981; McClain et al., 1985; Purdy, 1987; Burnett et al., 1989; Caress et al., 1992; Vera et al., 1990], mainly due to crack closure and precipitation of hydrothermal minerals. Hydrothermal cementation is also the favored interpretation for the off-axis increase in seismic velocity within the extrusive crust [Wilkens et al., 1991; Grevemeyer and Weigel, 1997].

Individually, no current seismic method is ideal for studying the shallow structure of oceanic crust. Although well-suited to analyzing gross crustal properties, conventional refraction experiments with surface sources lack spatial resolution in the uppermost kilometer, especially far from the on-bottom recording instruments, where energy turning in the shallow depths is not recorded. They also suffer from a noncommon depth point (CDP) geometry in that the source-receiver midpoint changes (and rays sample different depths) as the source ship steams along the refraction line. The CDP geometry and large source-receiver offsets of ESPs make them ideal for resolving crustal velocity structure, but only at one point. WAPs, although more limited in source-receiver aperture, are better suited to acquire velocity information in a spatially continuous fashion. However, the effectiveness of two-ship studies, like all sea-surface methods in deep water (including single-ship MCS and conventional 
refraction studies), is degraded by a seafloor reflection that can mask energy turning within the upper crust. They are also costly, and have therefore been performed at only a few locations. By placing both source and receiver near the seafloor, on-bottom seismics effectively remove the interference of the water wave, and allow crustal refractions to be recorded within a much shorter source-receiver offset. Yet, these benefits come at the cost of difficult logistics, a non-CDP geometry and sparse coverage (equal to the number of on-bottom receivers). Consequently, few on-bottom seismic experiments have been performed. Through their single-ship, CDP geometry, conventional multichannel studies are a cost-effective means of imaging the upper crust in a continuous fashion. Depending on water depth, however, this method's limited source-receiver aperture (generally $4 \mathrm{~km}$ or less) often fails to include refracted energy turning within the shallowmost crust [Harding et al., 1993; Carbotte et al., 1997]. As a result, it has historically been used to generate seismic "reflectivity" images of the subseafloor structure, but rarely to study crustal velocities.

In this study, we perform a semi-automated waveform inversion on conventional reflection data acquired between $17^{\circ} 18^{\prime} \mathrm{S}$ and $17^{\circ} 36^{\prime} \mathrm{S}$ on the East Pacific Rise. The goals of this study are two-fold. We wish to test the ability of single-ship multichannel data to provide detailed velocity information about extrusive crust in the near-ridge environment, where the seafloor is shallow enough to bring upper crustal refractions within the aperture of the multichannel streamer. Our second goal is to map the internal structure of the upper crust, including the geometry of layer $2 \mathrm{~A}$, both onand off-axis. By constructing a detailed and continuous map of upper crustal structure at several ages, we not only hope to chart the development of the extrusive crust, but also to discriminate between the various geological interpretations presented above. After introducing the study area in the following section, we present the reflection data and discuss how changes in upper crustal structure affect the layer $2 \mathrm{~A}$ and $2 \mathrm{~B}$ seismic events. We then outline the genetic algorithm-based approach used to invert the reflection data for upper crustal velocity structure. Finally, we present the results 
of these inversions in the form of cross-sectional and map views of the upper crust, and discuss their implications for the temporal and spatial distribution of processes affecting the extrusive section.

\subsubsection{The Study Area}

The East Pacific Rise between $17^{\circ} \mathrm{S}$ and $17^{\circ} 40^{\prime} \mathrm{S}$ is part of a $1150 \mathrm{~km}$ section of ridge between the Garrett transform and Easter microplate that is interrupted only by nontransform offsets [Lonsdale, 1989]. This area has been extensively mapped [Scheirer et al., 1996], and was the focus of two submersible dive programs [Renard et al., 1985; Auzende et al., 1996], a detailed seismic refraction/reflection survey (TERA) [Detrick et al., 1993], and the Mantle ELectromagnetic and Tomographic (MELT) experiment [Forsyth and Chave, 1994]. Plate motion models and magnetic anomaly picks predict this region to be spreading asymmetrically at $151-162 \mathrm{~mm} / \mathrm{yr}$ full-rate [Naar and Hey, 1989; DeMets et al., 1990]—among the fastest spreading rates in the world. The rise axis in this region is quite linear and varies in depth by less than $50 \mathrm{~m}$. Two small ridge-axis discontinuities are distinguished in Figure 5-1 by bathymetric lows at $17^{\circ} 05^{\prime} \mathrm{S}$ and $17^{\circ} 40^{\prime} \mathrm{S}$. They belong to a series of small $(<5 \mathrm{~km})$, left-stepping non-transform offsets extending from the Garrett transform to the large right-stepping $\mathrm{OSC}$ at $20.7^{\circ} \mathrm{S}$ [Lonsdale, 1989]. Based on magnetic and side-scan sonar data, Cormier and Macdonald [1994] propose that the total offset across this stairstep pattern was initially accommodated by a large discontinuity between $17^{\circ}$ and $17^{\circ} 30^{\prime} \mathrm{S}$, and that it was redistributed by a substantial replenishment of the magma reservoir during the past $1 \mathrm{Myr}$.

The Garrett-Easter ridge system reaches its shallowest, most inflated points at $17^{\circ} 27^{\prime} \mathrm{S}$ and $17^{\circ} 09^{\prime} \mathrm{S}$, both within this study area. These features indicate that this section of the EPR is magmatically robust, a result of enhanced melt generation in the upper mantle [Macdonald and Fox, 1988; Haymon et al., 1991; Scheirer and Macdonald, 1993]. Submersible dives show that the rise crest is largely unfaulted, 


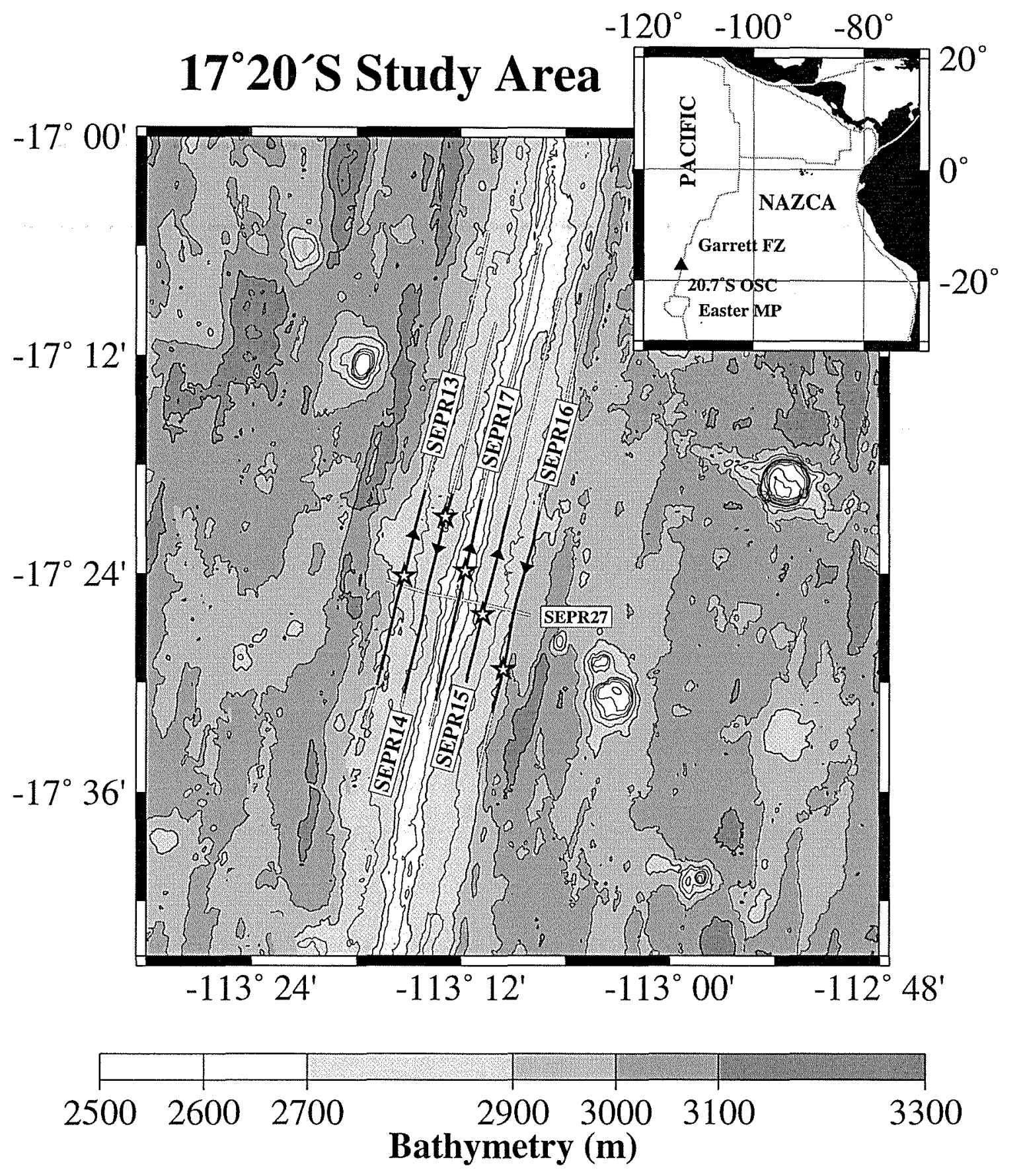

Figure 5-1: Bathymetric map of the $17^{\circ} 20^{\prime}$ S study area [after Scheirer et al., 1998] on the EPR (triangle in inset). Contour interval is $100 \mathrm{~m}$, except at the rise axis, where it is 50 m. Upper crustal structure was analyzed along $21-\mathrm{km}$ segments (black) of the five labeled seismic lines: one on-axis, and two on each side in 42- and 85-ky-old crust. Cross-axis line SEPR27 bisects the examined line segments and is discussed later. Stars locate the supergathers shown in later figures, and arrows show the direction each profile was acquired. Selected rise-axis discontinuities are labeled in the inset. 
hydrothermally active, and intermittently contains an axial summit depression up to $50 \mathrm{~m}$ deep and $100 \mathrm{~m}$ wide [Renard et al., 1985; Auzende et al., 1996]. By comparing recent submersible observations [Haymon et al., 1993] and rock analyses [Sinton et al., 1993] with their own, Auzende et al. [1996] suggest that the shallow rise axis at $17^{\circ} 27^{\prime}$ 'S may have erupted only a.few months to years prior to their dive program in 1993. This interpretation is consistent with seismic data, which image a locally thin ( $<10 \mathrm{~m})$, crystal-rich (i.e., melt depleted) magma sill [Hussenoeder et al., 1996a] that shoals by over $200 \mathrm{~m}$ beneath this region to within $800 \mathrm{~m}$ of the seafloor [Detrick et al., 1993; Mutter et al., 1995].

Hooft et al. [1997a] and Carbotte et al. [1997] have previously examined the geometry of layer $2 \mathrm{~A}$ in this area using conventionally processed reflection profiles. Both document a significant, yet variable, off-axis thickening of layer $2 \mathrm{~A}$, but they disagree on the absolute depths of this horizon, especially on-axis. Carbotte et al. [1997] also document a variability in the width of the extrusive accumulation zone (2.9-7.4 $\mathrm{km}$ ), with the broadest area of volcanic emplacement overlying the magma sill at its shallowest point. In contrast to these studies, we examine selected lines along, and parallel to, the rise crest. Our approach offers less cross-axis resolution, but yields detailed information on both the velocity and reflectivity structure along the profiles and in depth.

\subsection{Data Collection and Analysis}

As part of the 1991 TERA experiment, multichannel seismic data were collected in this region both on- and off-axis. Reflection data from the single-ship component of the survey were acquired by the R/V Maurice Ewing's 160-channel, 4-km-long digital streamer. The seismic source was generated by port and starboard halves of a 20 -gun, 8385 -cubic-inch airgun array, alternately firing every $10 \mathrm{~s}$ to yield a $25 \mathrm{~m}$ shot spacing and eighty-fold coverage. 
Five profiles in this study area were chosen for a detailed velocity analysis of the upper crust: one along-axis, and lines along 42- and 85-ky-old crust on either side of the spreading center ( 3 and $6 \mathrm{~km}$ off-axis, respectively). Only lines following the morphotectonic grain were chosen for analysis, since bathymetric and subsurface variations are less extreme in this direction. Data gathers from these lines exhibit energy turning beneath layer $2 \mathrm{~A}$ in front of the seafloor reflector at large ranges, and were chosen for this reason (see Fig. 5-2). Constant offset stacks (COS), or supergathers, were constructed by summing $80 \mathrm{CDP}$ gathers to enhance the signal to noise ratio. Traces were band-pass filtered between 5 and $40 \mathrm{~Hz}$ to remove the lowfrequency strumming of the streamer and spurious high-frequency noise. Twenty-one supergathers (SG) were constructed in this fashion at $1 \mathrm{~km}$ intervals along each of the five seismic profiles, generating a 105-point grid in map view centered on the rise axis at $17^{\circ} 24^{\prime} \mathrm{S}$. A velocity analysis was performed at each grid location. Figure 5-2 displays a representative supergather from each line.

Figure 5-3 shows a synthetic 1-D model for young upper oceanic crust at a fast spreading center and the associated arrivals that are identifiable in the data (Fig. 52). We define layer $2 \mathrm{~A}$ to be both the surficial low-velocity layer and the underlying high-gradient transition. This is consistent with earlier definitions of layer $2 \mathrm{~A}$, and correlates well with CDP sections, in which the layer $2 \mathrm{~A}$ event is generated by energy turning at the base of the velocity gradient. This gradient layer bends energy back toward the source, generating a triplication in arrival times and concentrating energy into a high-amplitude arrival at the caustic (Fig. 5-3b,c). The location and character of these events is highly dependent on the water depth, the velocity and thickness of layer $2 \mathrm{~A}$, the gradient zone and layer $2 \mathrm{~B}$, as well as the presence of secondary structures. For example, a deeper seafloor would shift arrivals to greater times and farther ranges, since energy at equivalent incidence angles would travel farther vertically and horizontally. A thickening of the surficial low-velocity layer would produce a similar result, but only for the gradient and layer $2 \mathrm{~B}$ events. Differences in these two pa- 

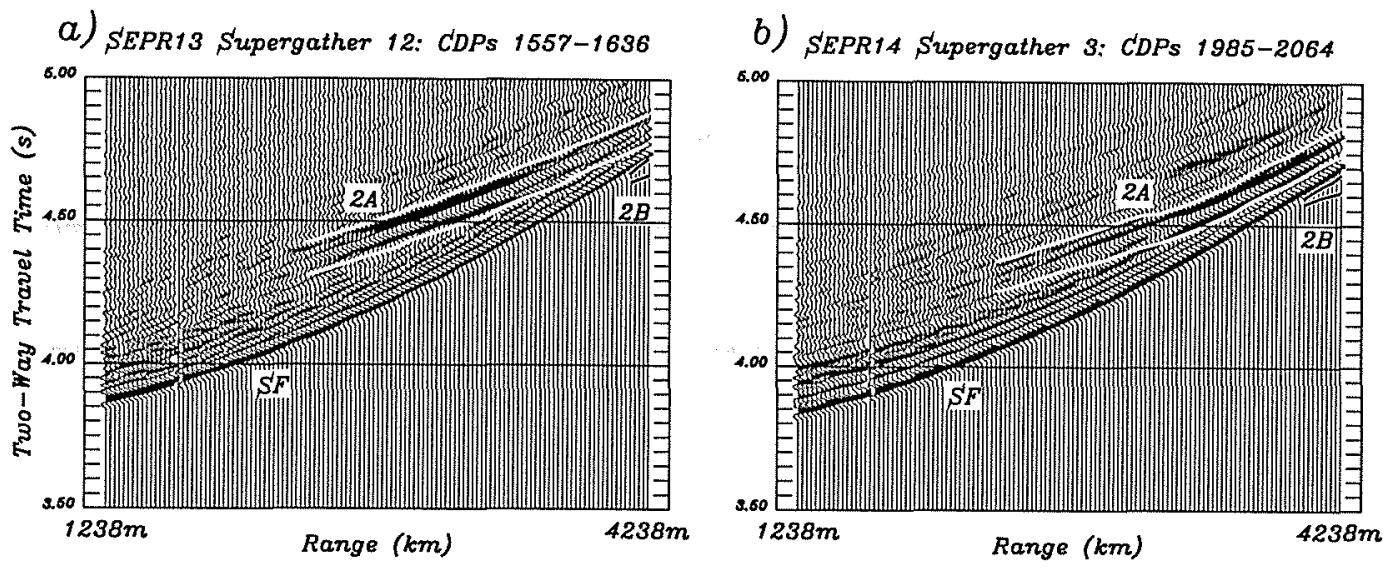

C) SEPR17 Supergather 14: CDPS 1676-1755
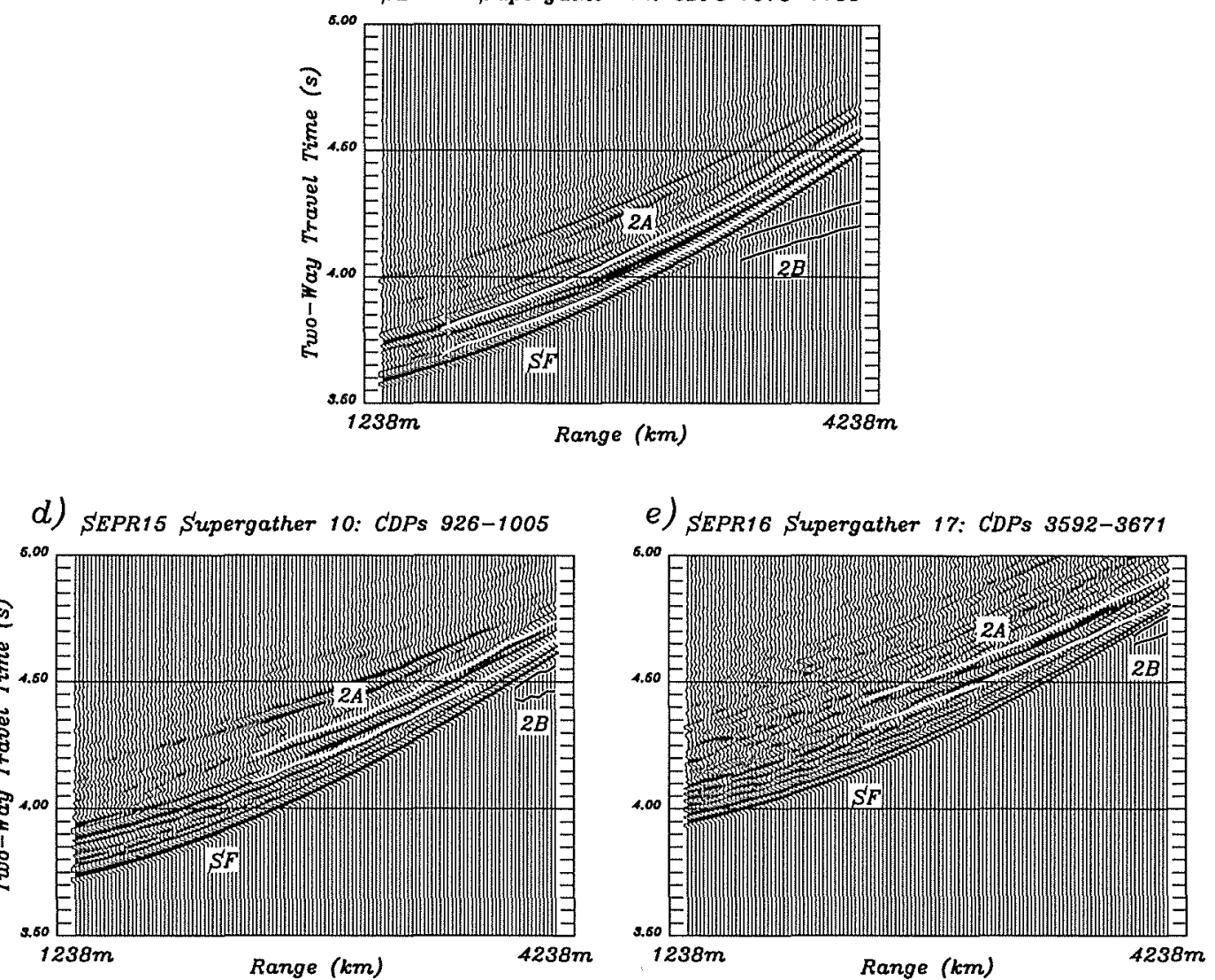

Figure 5-2: Representative supergathers for a) SEPR13, $6 \mathrm{~km}$ west of the rise axis, b) SEPR14, $3 \mathrm{~km}$ west, c) SEPR17, along-axis, d) SEPR15, $3 \mathrm{~km}$ east, and e) SEPR16, $6 \mathrm{~km}$ east. All data are plotted at the same amplitude scale. The $2 \mathrm{~A}$ and $2 \mathrm{~B}$ events are outlined by white and black curves, respectively. Synthetic seismograms were compared to the data only within these windows. Note how the increased time delay of the layer $2 \mathrm{~A}$ event off-axis and the greater water depth causes the layer $2 \mathrm{~B}$ event to cross the seafloor reflection at larger ranges. No 2B energy was recorded prior to the seafloor reflection (SF) in a). 

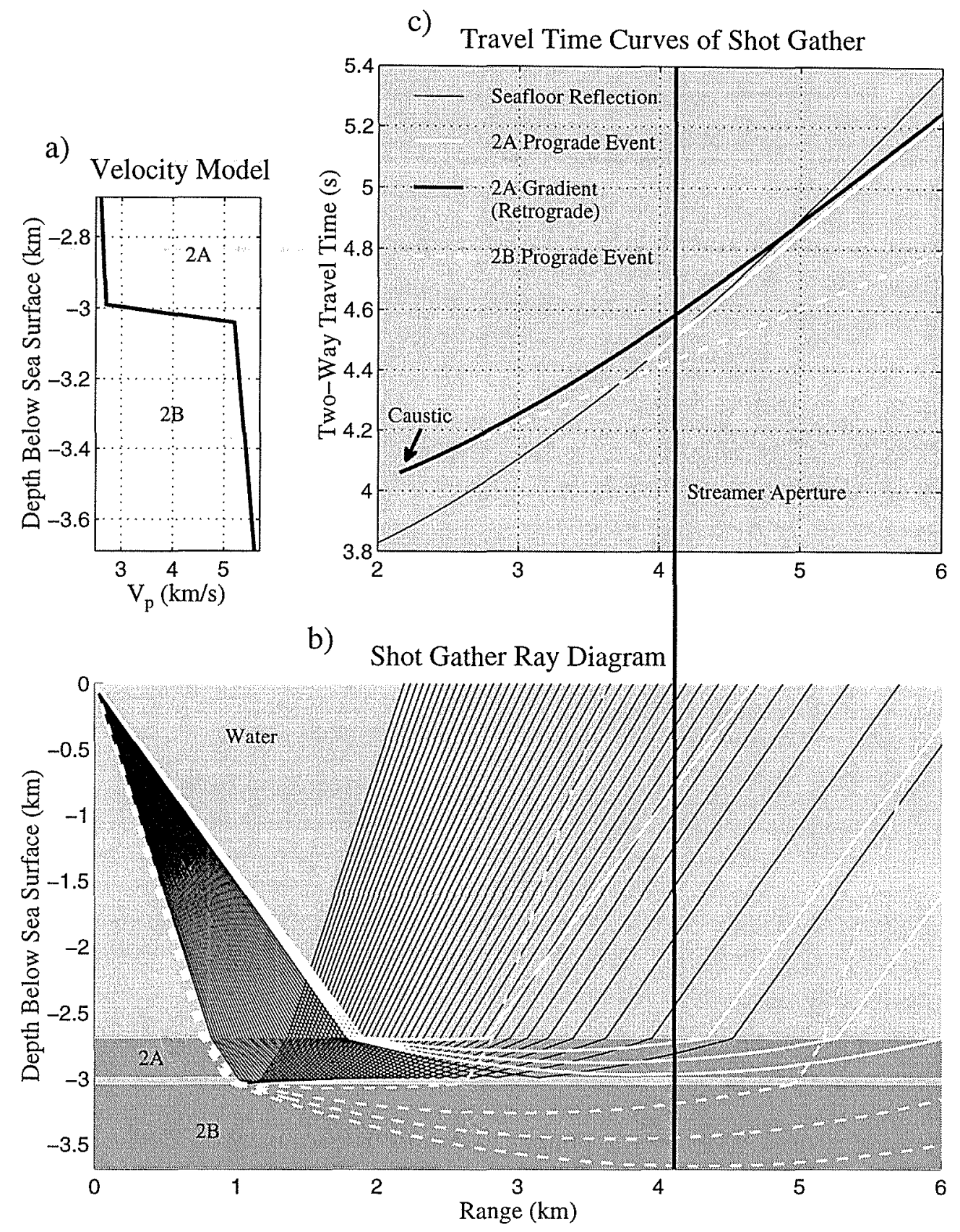

Figure 5-3: a) Canonical P-wave velocity model for young oceanic crust in which a lowvelocity volcanic layer $(2 \mathrm{~A}$ ) overlies a steep velocity transition (also $2 \mathrm{~A}$ ) to a higher velocity dike section (2B), b) corresponding ray diagram, and c) travel time curves for a shot gather geometry (equivalent to a CDP geometry when velocity structure is 1-D). Energy traveling through the uppermost layer (white), the radient zone (thick black), and layer $2 \mathrm{~B}$ (dashed) generates a distinct triplication pattern. A thick vertical line represents the maximum source-receiver distance in the data. Rays are plotted at equal slowness increments to show the increased ray density (i.e., focused energy) near the caustic at $2.15 \mathrm{~km}$ range. 
rameters account for most of the variation between the supergathers in Figure 5-2. An increase in crustal seismic velocity would produce the opposite effect by bending energy closer to the source and lessening the time spent within the crust. Refractions provide excellent constraints on seismic velocities, since the inverse slope of a refracted arrival represents the velocity at that ray's turning point (note the curved gradient arrival and linear prograde arrivals in Figure 5-3c).

This study takes advantage of both the common depth point geometry and continuous mapping that single-ship MCS data offer. By constructing data gathers that record energy turning at a single midpoint, we drastically reduce the lateral extent over which the crust is sampled, thereby allowing each study site to be better approximated by a model varying only with depth. In Figure 5-3b, for example, energy turning at the base of layer $2 \mathrm{~A}$ (the primary focus of this study) and recorded by a 4- $\mathrm{km}$ streamer laterally samples $1.1 \mathrm{~km}$ of the transition zone in a shot gather geometry, whereas only $0.2 \mathrm{~km}$ is sampled in CDP mode. CDP sorting equivalently reduces the amount of seafloor this event samples. For the end-member case of a velocity discontinuity, a CDP gather would sample the transition only at a single point. Although stacking adjacent gathers over a 1-km interval (80 CDPs) slightly degrades our assumption of one-dimensionality, the spatial averaging $(<2 \mathrm{~km})$ is small enough that the overlap in ray coverage between grid locations is less than $50 \%$.

Our analysis method is essentially an iterative pre-stack velocity inversion, whereby synthetic seismograms are calculated from model velocity functions and compared to the data. Performing our inversions in the pre-stack domain has several advantages over conventional post-stack velocity analyses. Both Harding et al. [1993] and Carbotte et al. [1997] caution that conventional CDP processing methods do not guarantee the successful imaging of upper crustal structure. One source of error is the use of stacking velocities, which assume that all events, including refracted energy, follow hyperbolic reflection trajectories. The common use of geologically approximate velocity models, not constrained by the CDP data, to convert the resultant seismic 
profiles from two-way travel time to depth also results in the possible mislocation of important structures. No conversion to depth is necessary following pre-stack velocity analysis. The output velocity model is already a continuous depth representation of reflectivity (or impedence contrast). In addition, the success of conventional velocity analysis methods depends on the existence of multiple reflectors, a trait oceanic crust seldom exhibits. Waveform inversion not only models velocity discontinuities and gradients, but also uses both travel time and waveform information to provide a higher degree of resolution. Modeling refraction triplications is a highly nonlinear problem. For this reason, and to eliminate human errors associated with hand-picking events, we automate this process through the use of a genetic algorithm.

The genetic algorithm (GA) was employed to search velocity-depth model space and construct new velocity models at each successive iteration. For a discussion on its specific implementation, see Appendix A. Synthetic seismograms were computed using the WKBJ method [Chapman, 1978], and convolved with a source waveform. In the absence of a recorded far-field waveform, our source was derived from stacking the near-offset seafloor reflections of all on- and off-axis supergathers. The resulting WKBJ synthetic seismograms then included the effects of surface interaction near the source and receiver. Synthetics were compared to the data seismograms only within two windows surrounding energy emanating from the upper crust (Fig. 52): the layer $2 \mathrm{~A}$ event, which represents energy being turned by the high-gradient $2 \mathrm{~A} / 2 \mathrm{~B}$ boundary; and the layer $2 \mathrm{~B}$ event, which records energy turning within the low-gradient layer directly below this boundary. Energy turning in the surficial lowvelocity layer generally falls outside the 4 -km aperture of the hydrophone streamer (Fig. 5-3). Yet, the velocity structure of this layer is still resolvable, since all energy turning below this section must also travel through it. 


\subsection{Results}

The analysis of all five profiles was begun by choosing a supergather that exhibits a clearly defined layer $2 \mathrm{~A}$ and $2 \mathrm{~B}$ event. A reasonable starting model was constructed for this location by hand-fitting the travel times generated by a four-node velocitydepth function (such as in Fig. 5-3). After applying the GA to this supergather, the mean final model was treated as the mean starting model for the two adjacent locations. This process was repeated until the ends of the profile were reached. Depths greater than $1 \mathrm{~km}$ below the seafloor were not included in our models, since refractions sampling those levels generally return to the sea surface outside the streamer aperture (Fig. 5-3). Table 5.1 summarizes our inversion results.

\subsubsection{Along-Axis Profile: SEPR17}

After a careful examination of the data, supergather 5 was chosen to be our on-axis starting location. Hand-fitting the travel times in this gather resulted in a starting model composed of a 150-m-thick, low-velocity layer $(2.1-2.2 \mathrm{~km} / \mathrm{s})$ overlying a single, $50-\mathrm{m}$-thick transition to a layer $2 \mathrm{~B}$ velocity of $5.4 \mathrm{~km} / \mathrm{s}$. The analysis of this line was then automated by the GA-driven waveform inversion. Our results show that, although our simple input model correctly predicts the velocities within each layer, the character of the $2 \mathrm{~A} / 2 \mathrm{~B}$ transition is more complicated than a single gradient (Figs. 5-4 \& 5-5). At every point along line SEPR17, the best fitting models are those that include two velocity transitions separated by a zone of moderate velocity increase. These velocity-depth functions illustrate the GA's ability to find solutions far from the starting model.

The shallowmost crust along this section is generally characterized by a uniform, 70 to 100 -m-thick low-velocity interval overlying an extremely steep gradient. Energy turning within the surficial layer falls outside the streamer aperture (Fig. 5-3, due to its low velocity, shallow vertical velocity gradient and the water depth. Its 
Table 5.1: Summary of Inversion Results

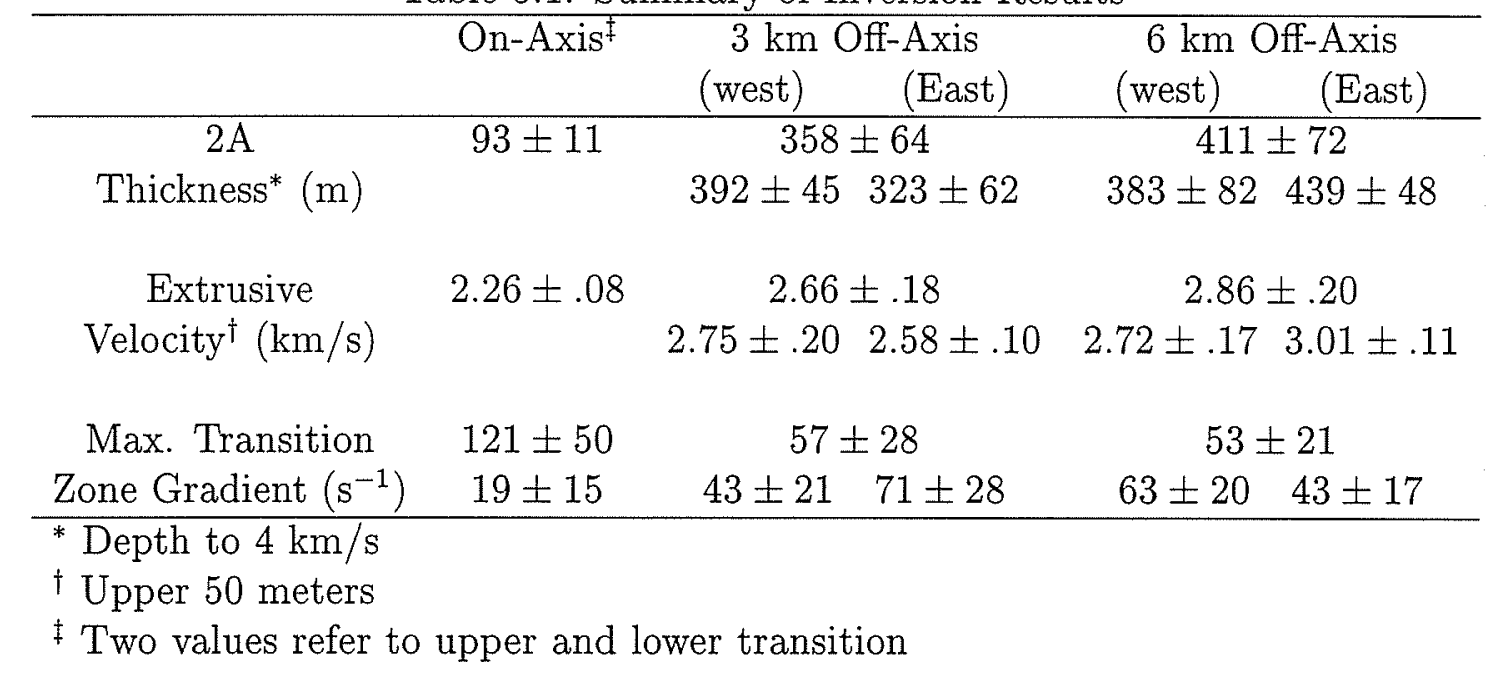



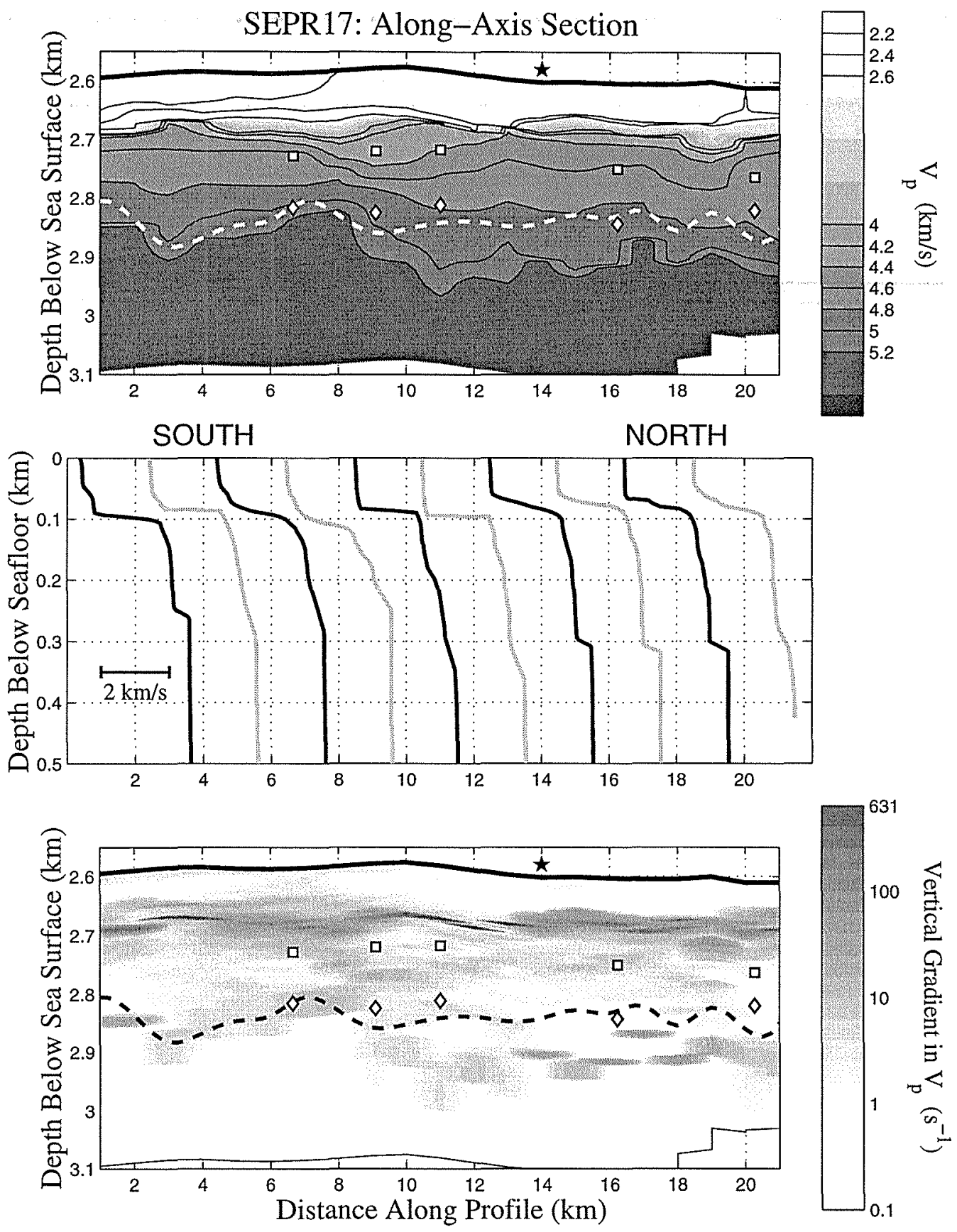
Figure 5-4: (previous page) Seismic cross-section of the along axis profile, SEPR17. (top) Contoured seismic velocity in the upper 500 meters obtained by the GA at $1 \mathrm{~km}$ intervals. Contour lines are keyed to their respective velocities in the colorbar. A star (see Figure 5-1) marks the location of the representative supergather shown in Figure 5-2c. A dashed line marks the base of layer $2 \mathrm{~A}$ from picking the travel times of conventionally processed along-axis seismic profiles [E. E. E. Hooft, unpublished data]. These picks were converted to depth in this study by using the corresponding velocity-depth functions obtained by the GA. Symbols represent published layer $2 \mathrm{~A}$ thicknesses from similarly picking five cross-axis seismic profiles. Squares are from Carbotte et al. [1997]; diamonds are from Hooft et al. [1997a]. (center) A sampling of the mean final velocity-depth functions (Eq. A.11] that make up the upper panel. Models at evenly numbered distances along the profile are shown versus depth below seafloor (alternating grey and black for clarity). (bottom) Grey-shaded velocity gradients within the uppermost crust. The symbols, dashed line and spatial scale are the same as in the upper panel. Note the nearly uniform thickness of low-velocity crust overlying a 150 to 250 -m-thick section of moderate velocity gradients, beneath which a second jump in velocity often occurs (e.g., at $2,14,16$ and $18 \mathrm{~km}$ ).

Figure 5-5: (next page) GA waveform inversion results for the on-axis supergather 14, shown in Figure 5-2c and marked by a star in Figures 5-1 and 5-4. a) Waveform comparison between the best fitting model (thick traces) and the data (thin traces). Every fourth trace within the $2 \mathrm{~A}$ and $2 \mathrm{~B}$ windows is shown, since only these were used in calculating the misfit for all on-axis supergathers. Synthetic traces are shifted for optimal fit in the lower plot, with the row of numbers (zeros in this case) corresponding to the number of samples shifted. A thick vertical line separates the layer $2 \mathrm{~A}$ and $2 \mathrm{~B}$ events. Note the small misfit $(\phi=14.1$, see Appendix A.2). b) Travel time curves for the mean final model, superimposed on the data seismograms. The layer $2 \mathrm{~A}$ and $2 \mathrm{~B}$ windows are shaded grey. c) Mean final (thick line) and best (thin line) velocity model for this location. The two standard deviation error bound is shaded grey. The increased uncertainty with depth is partly a result of model parameterization. Note the models' double-step character, which manifests itself as a small triplication in the travel times of refracted energy crossing the seafloor reflection. 
a) SEPR17 Supergather 14: Trace Comparison with Best Model (Unshifted) [ $\phi=14.092]$

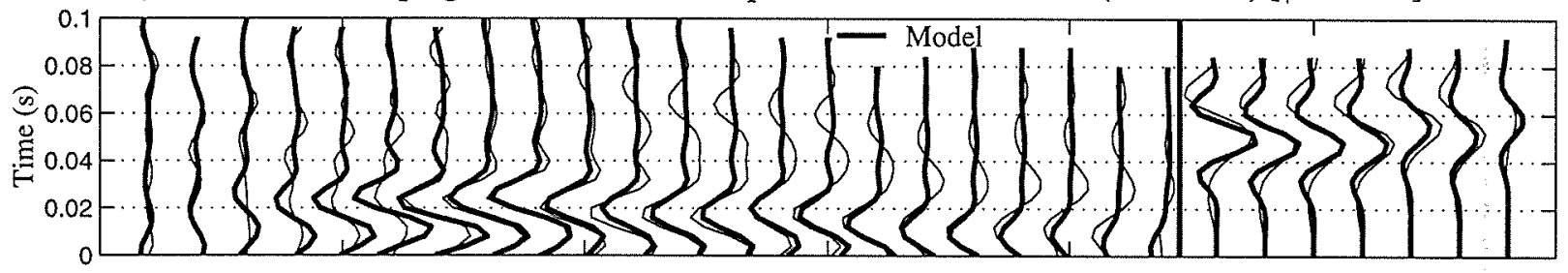

Trace Comparison (Shifted)

N

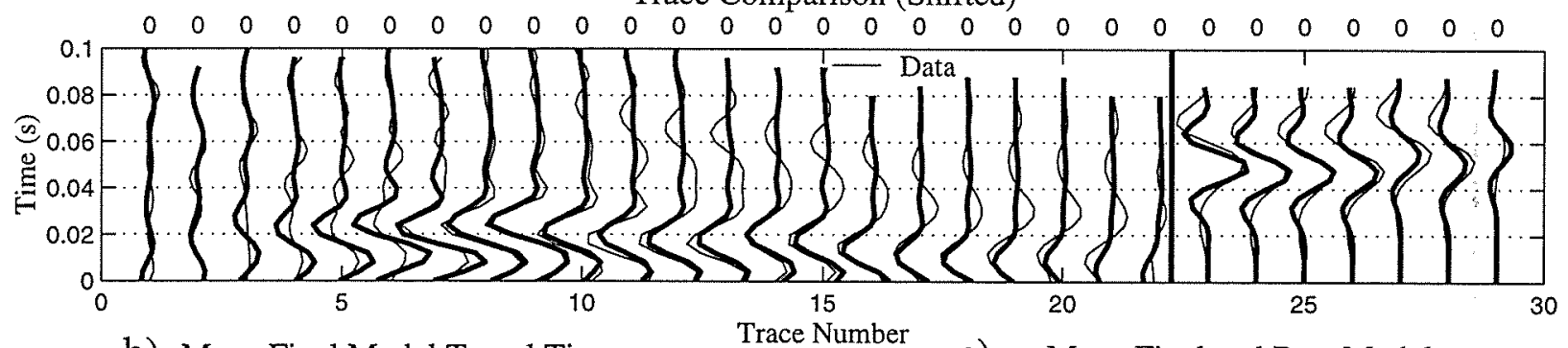

b) Mean Final Model Travel Times

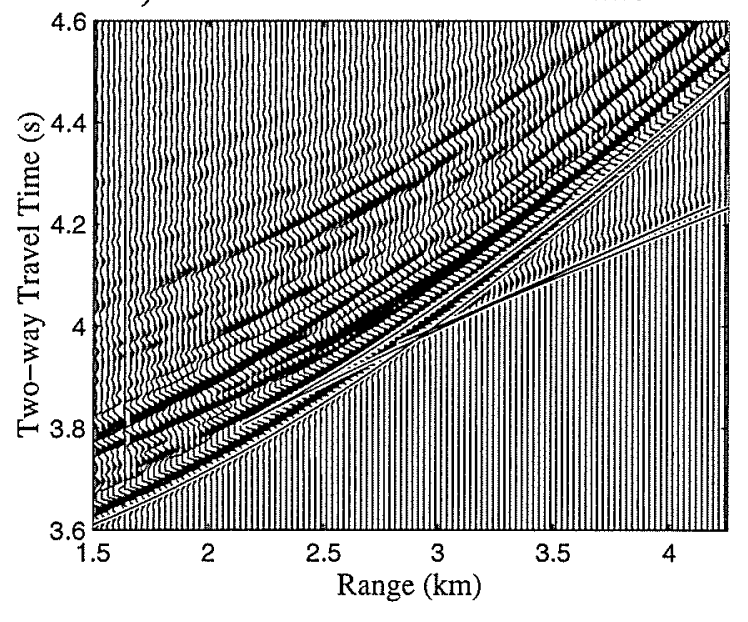

c) Mean Final and Best Model

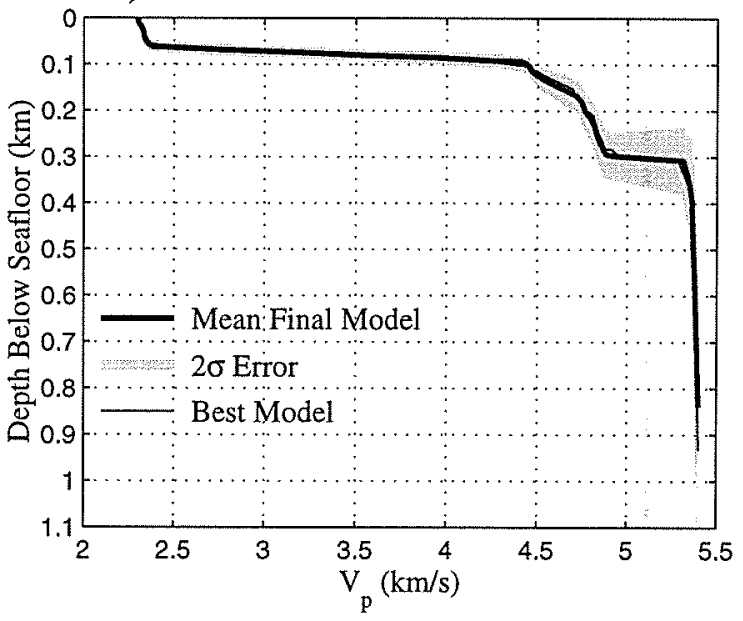


fine-scale structure is therefore not constrained by the data. However, the average velocity and remarkably uniform thickness of this layer is well-constrained, because it controls where the retrograde branch from the underlying transition zone re-enters the streamer aperture. The transition spans $2 \mathrm{~km} / \mathrm{s}$ within only a few tens of meters. Gradients above $100 \mathrm{~s}^{-1}$ are reached in a majority of locations, but are concentrated near the center and southern end of the profile. The short depth interval over which this transition occurs is constrained by the shallow curvature of the retrograde $2 \mathrm{~A}$ branch, and its extension back to near-offset traces-both indicators of sharply refracted energy (Figs. 5-2c \& 5-3).

Beneath this feature lies a 150 to 210 -m-thick region of moderate velocity increase $\left(\sim 2 \mathrm{~s}^{-1}\right)$, overlying a second, smaller velocity jump. This pattern is manifested to varying degrees along this profile, with remarkably sharp transitions predicted for supergathers 1-2 and 14-19. By examining in detail the modeling results from one of these locations (Fig. 5-5), it is evident that the double-step structure produces a small triplication of travel times between the main caustic (behind the seafloor reflection) and the $2 \mathrm{~B}$ branch. This solution is made possible by the GA's ability to simultaneously model both amplitude and travel times. The triplication is necessary to connect the amplitude-constrained location of the main caustic to the $2 \mathrm{~B}$ branch, and still match the slope of this pre-seafloor arrival. The greater the mismatch between the caustic and the backward projection of the $2 \mathrm{~B}$ branch, the greater the velocity jump necessary to generate the correct triplication. Figure 5-5 illustrates how the single-gradient model is modified to produce an excellent fit to the data in amplitude, travel time and waveform. Part of the energy from this transition to layer $2 \mathrm{~B}$ velocities is masked by the seafloor reflection. As a result, the error bound for the lower transition is significantly higher than for the upper gradient.

Layer $2 \mathrm{~B}$ velocities near $5.4 \mathrm{~km} / \mathrm{s}$ are reached within 240-380 meters of the seafloor, below which the crust remains relatively uniform in velocity. For the same reasons as for the surficial low-velocity layer, the detailed structure of this layer's 
lower portion is unconstrained by the data. However, no events generated by velocity jumps within this interval are evident. Energy from such features would be bent back within the aperture of the streamer and detected as high-amplitude events. Seismic evidence for a high-velocity conductive lid overlying the axial magma chamber (AMC) has been recorded in wider aperture data [Vera et al., 1990; Tolstoy et al., 1997; Singh et al., 1998]. We therefore suggest that the relatively uniform velocities observed in the top portion of layer 2B may extend down to the conductive lid above the AMC.

\section{$5.3 .23 \mathrm{~km}$ Off-Axis: SEPR14 \& SEPR15}

Lines SEPR14 and SEPR15 image 42-kyr-old crust. The starting supergather for these lines was located at 3 and $10 \mathrm{~km}$ along their lengths, respectively. Handmodeling yielded a single-gradient model at both starting points, with a depth to the transition significantly deeper than on-axis. The solutions obtained by the automated inversion retain the single-gradient structure, but vary the depth and sharpness of the transition considerably (Figs. 5-6 \& 5-8).

Velocities within the uppermost layer are consistently $0.3-0.5 \mathrm{~km} / \mathrm{s}$ higher $3 \mathrm{~km}$ off-axis than along the rise crest. This change is manifested in the data by a reduction in slope of the retrograde $2 \mathrm{~A}$ branch at large offsets (Figs. 5-7b \& 5-9b). The increased travel times that would be associated with this thickening section are initially offset by its higher velocities, and become evident only as the caustic is approached. With few exceptions, the caustic along lines SEPR14 and SEPR15 is located at farther ranges than it is on-axis. This indicates that the $2 \mathrm{~A} / 2 \mathrm{~B}$ velocity transition is more gradual. Maximum gradients typically approach $50-60 \mathrm{~s}^{-1}$. The increased distance between the caustic and the seafloor event reflects the significant off-axis deepening of the $2 \mathrm{~A} / 2 \mathrm{~B}$ transition. This transition commonly occurs $300-400$ meters below the seafloor, and overlies a relatively uniform layer 2B. Velocities in this layer are constrained by slope of the $2 \mathrm{~B}$ event to gradually approach $5.6 \mathrm{~km} / \mathrm{s}$, slightly faster than at the rise axis. 

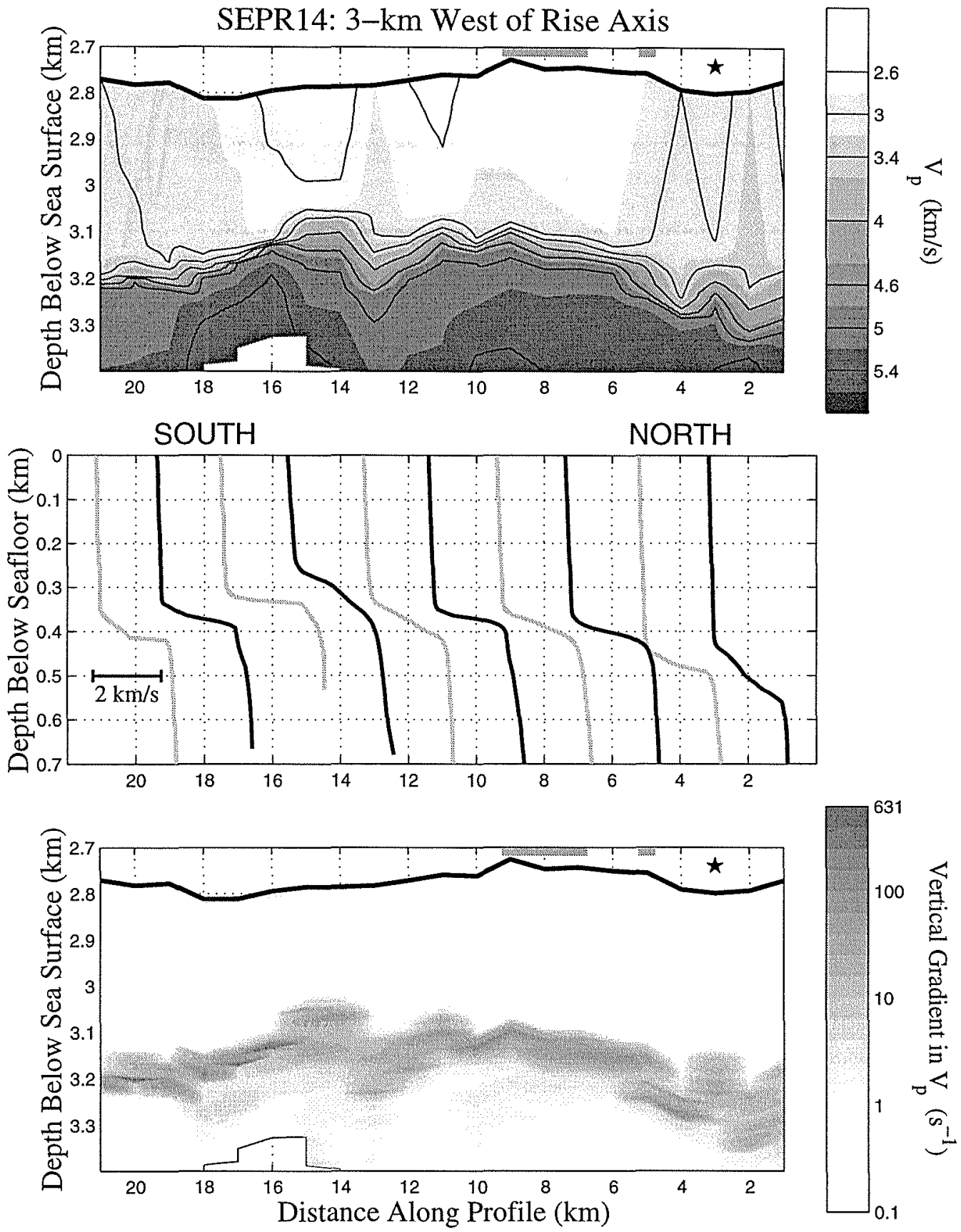

Figure 5-6: Same as Figure 5-4, except for the profile $3 \mathrm{~km}$ west of rise axis: SEPR14. The profile corresponds to 42 -ky-old crust and is plotted to $\sim 700$ meters below seafloor. The star marks the location of the supergather shown in Figure 5-2b. In this profile, as in all off-axis profiles, a single high-velocity gradient is sandwiched between two zones of nearly constant velocity. This transition is significantly deeper and generally thicker than the upper velocity jump in the along-axis profile of Figure 5-4. Gaps exist (grey bars) where the data were not reliable enough to accurately construct a velocity model. 

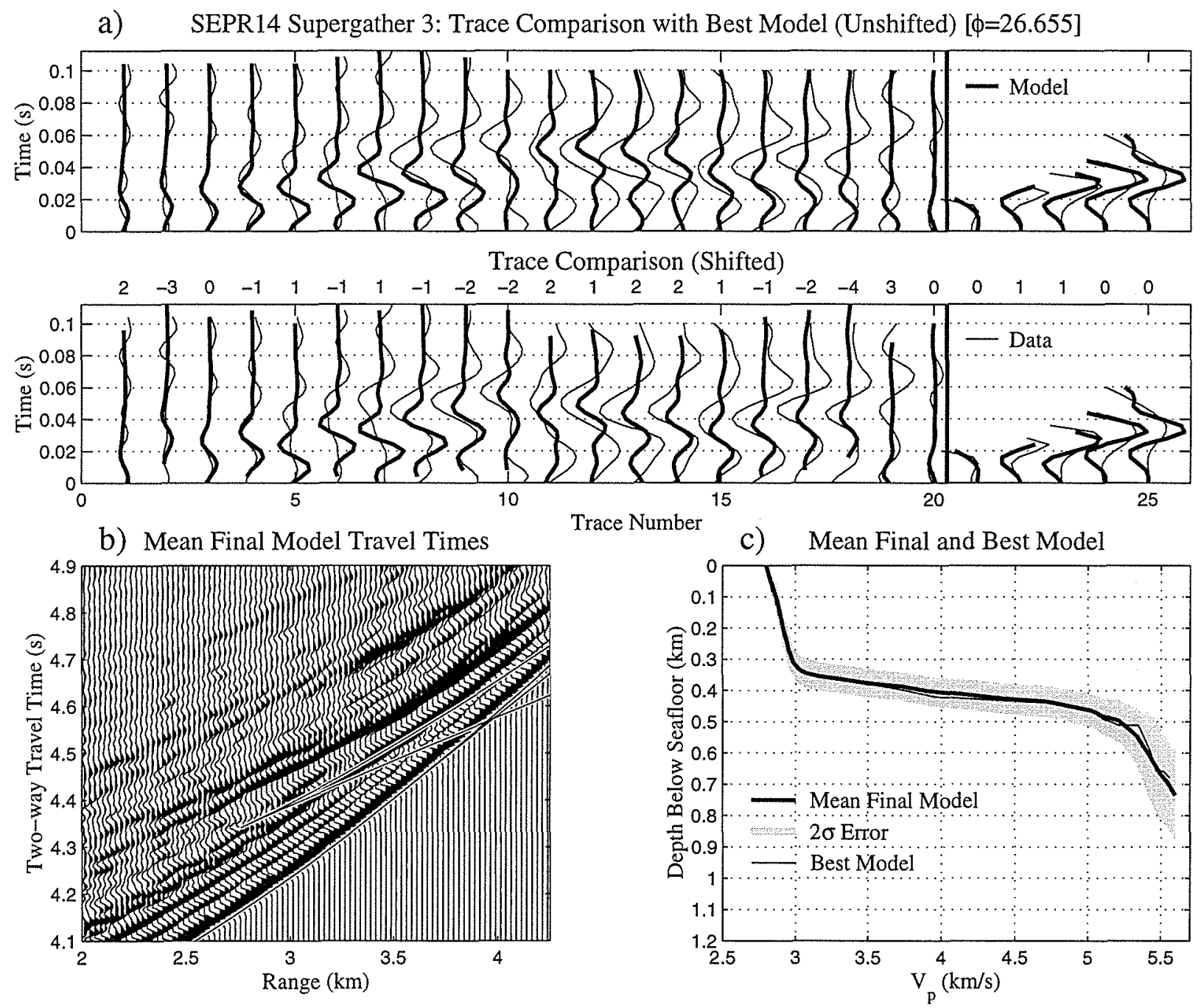
Figure 5-7: (previous page) Same as Figure 5-5, except for the $3 \mathrm{~km}$ off-axis supergather 3, shown in Figure 5-2b and marked by a star in Figures 5-1 and 5-6. Every fourth trace in the $2 \mathrm{~A}$ window and every second trace in the $2 \mathrm{~B}$ window was used in calculating the misfit for all off- axis supergathers. Although the travel times of the mean final model in $\mathrm{b}$ ) show that the synthetic travel times match the data well, the width of the event (i.e., frequency content) is not matched. This results in a moderate misfit and, consequently, a larger error bound in c) than for on-axis locations. Reasons for this mismatch are discussed in the text. Note the single-step character of the models in c).

Mean final models for line SEPR15 fit the data better than those for SEPR14, but the misfits along both are consistently higher than for on-axis profile SEPR17. Consequently, the error bounds surrounding the mean final models are larger (compare Figs. 5-5, 5-7 \& 5-9). The decrease in fit off-axis is likely to be caused by out-of-plane energy (not modeled by the inversion process) partially interfering with the windowed events. Off-axis, the tectonic fabric is more pronounced, and the seafloor and $2 \mathrm{~A} / 2 \mathrm{~B}$ boundaries are no longer at a depth minimum directly beneath the ship. Another possible source of higher misfits is the increased intrinsic attenuation $(Q)$ associated with a thicker extrusive section. Attenuation is not included in the WKBJ forward calculation. Recent studies at the EPR estimate extremely low $Q$-values within the extrusives $\left(11 \leq Q_{p} \leq 22\right)$ [Christeson et al., 1994b], and an increased attenuation slightly off-axis, due to in-situ hydrothermal processes [Wilcock et al., 1992]. Intrinsic attenuation is frequency dependent. A thicker, possibly more attenuative, off-axis volcanic section would return longer period events with a more limited frequency content, as evidenced by Figures 5-7a and 5-9a.

\section{$5.3 .36 \mathrm{~km}$ Off-Axis: SEPR13 \& SEPR16}

The analysis of lines SEPR13 and SEPR16 began with a single-gradient input model at the 13 and $15 \mathrm{~km}$ mark, respectively. The analysis results are shown in Figures 5-10 through 5-13. The inversions yield a distinct difference between these profiles in that the average extrusive velocity and layer $2 \mathrm{~A}$ thickness is greater to the east of 

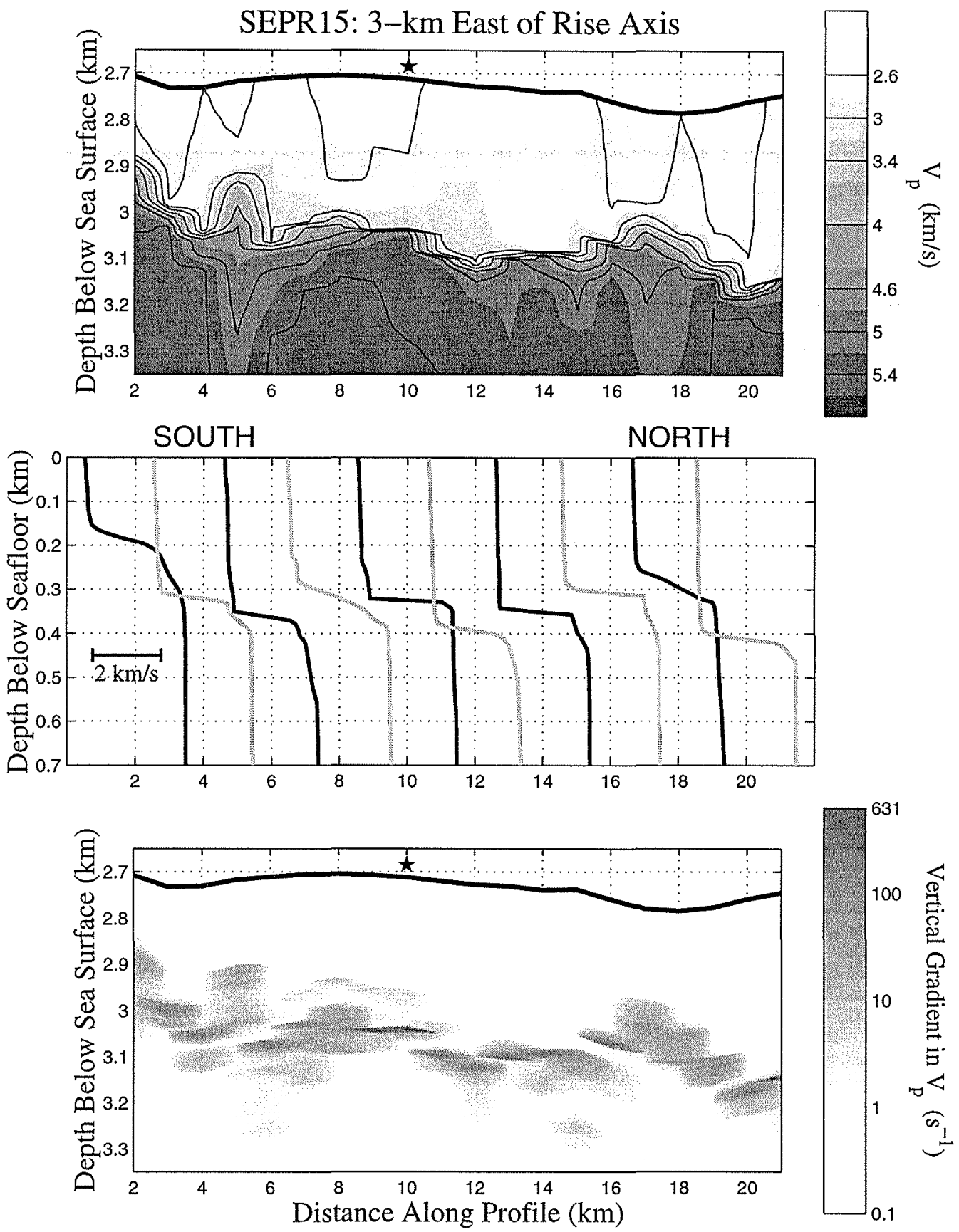

Figure 5-8: Same as Figure 5-6, except for the profile $3 \mathrm{~km}$ East of rise axis: SEPR15. The star marks the location of the supergather shown in Figure 5-2d. 
a) SEPR15 Supergather 10: Trace Comparison with Best Model (Unshifted) [ $\phi=20.389]$

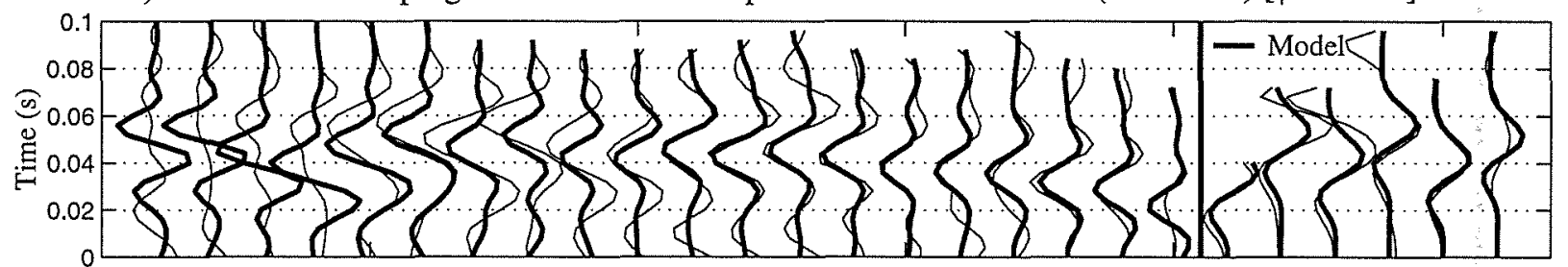

Trace Comparison (Shifted)

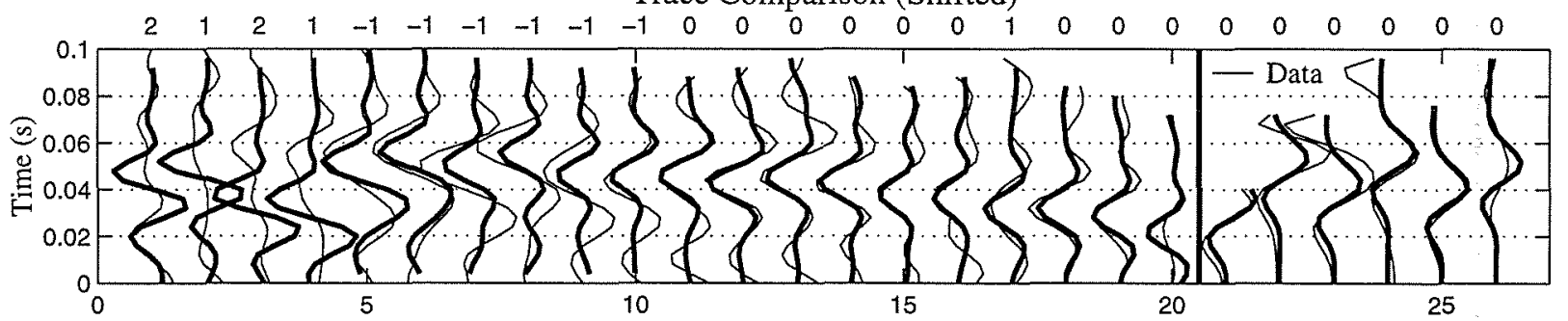

b) Mean Final Model Travel Times

Trace Number

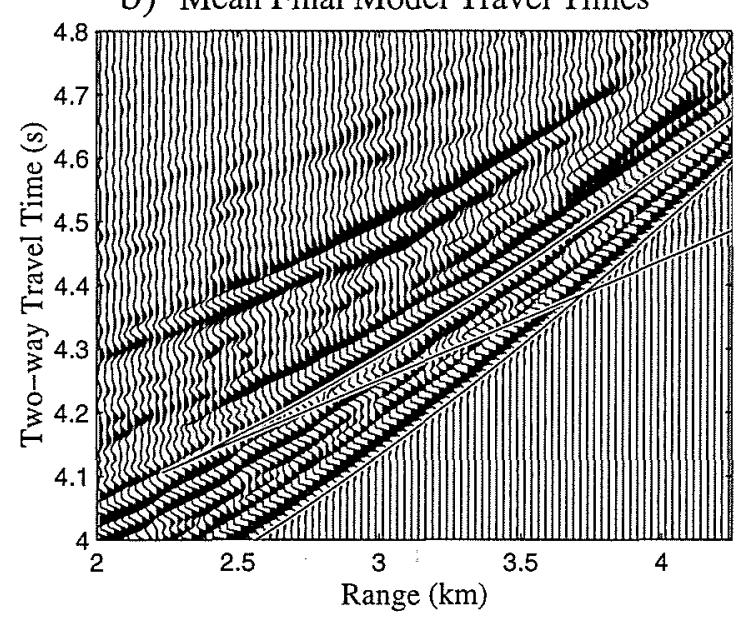

c) Mean Final and Best Model

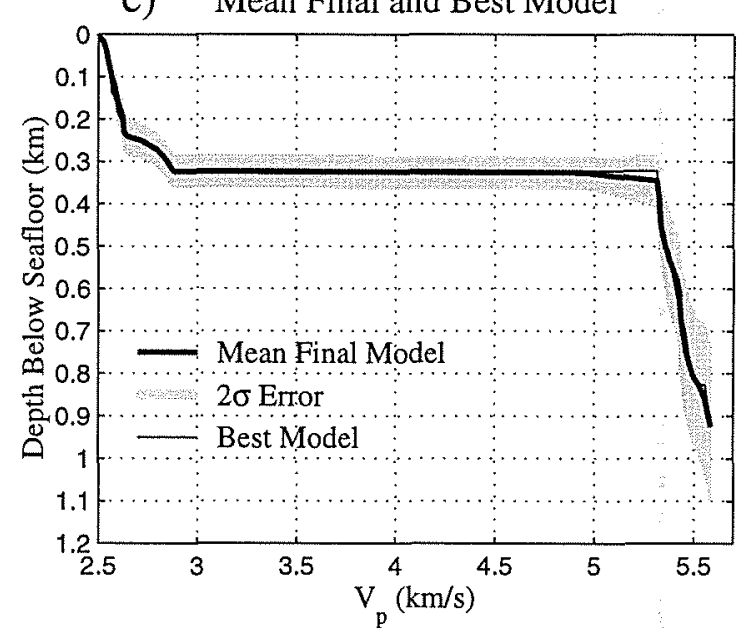

Figure 5-9: Same as Figure 5-7, except for the $3 \mathrm{~km}$ off-axis supergather 10, shown in Figure 5-2d and marked by a star in Figures 5-1 and 5-8. The misfit of the best fitting model is low, and it correlates well with the data. 
the rise crest than to the west. Crustal age along these profiles is $\sim 85 \mathrm{kyr}$.

High extrusive velocities along SEPR16 are constrained not only by the long-range character of the $2 \mathrm{~A}$ retrograde branch, but also by the appearance of the prograde $2 \mathrm{~A}$ event within the data aperture (e.g., Fig. 5-13b). This event records energy turning immediately beneath the seafloor. Figure 5-13 illustrates how the interference of this event with the $2 \mathrm{~B}$ branch slightly modulates the amplitude and period of waveforms in front of the seafloor reflection. Only along line SEPR16, and a small portion of SEPR13, are the seafloor velocities high enough to be directly and unambiguously recorded in the data. With the exception of the southern half of SEPR13, both lines reflect additional thickening of the low-velocity layer between 3 and $6 \mathrm{~km}$ off-axis. The velocity transition lies at a relatively uniform depth along SEPR16, whereas line SEPR13 exhibits an almost bimodal character, with shallow depths to the south. Although gradients within the transition zone remain relatively high along both profiles, a slight decrease is resolved between 3 and $6 \mathrm{~km}$ to the East. Velocities in layer $2 \mathrm{~B}$ increase to $5.8 \mathrm{~km} / \mathrm{s}-0.2 \mathrm{~km} / \mathrm{s}$ higher than the near-axis profiles, and $0.4 \mathrm{~km} / \mathrm{s}$ higher than on-axis.

In addition to along-axis variations, cross-axis trends in upper crustal structure can be examined by combining the results from all five profiles. When making such a comparison across horizons of varying depth and structure, it is important to objectively define the crustal measures to be examined. By imposing as few preconceived notions as possible about the geology in our analysis, we hope to generate an unbiased picture of the shallow crustal structure in this region. Figure 5-14 is a contoured map view of the extrusive velocities in our $6 \times 20 \mathrm{~km}$ study area. Each datum is the mean velocity across the upper $50 \mathrm{~m}$, and thereby includes only material within the low-velocity layer, regardless of location. The off-axis increase in velocity is clearly shown, as is the apparent cessation of this trend to the west. Along-axis, the extrusives velocities become distinctly faster to the north. This trend is not reflected along the flanks of the rise crest, which exhibit a rather uniform pattern to the east and a 

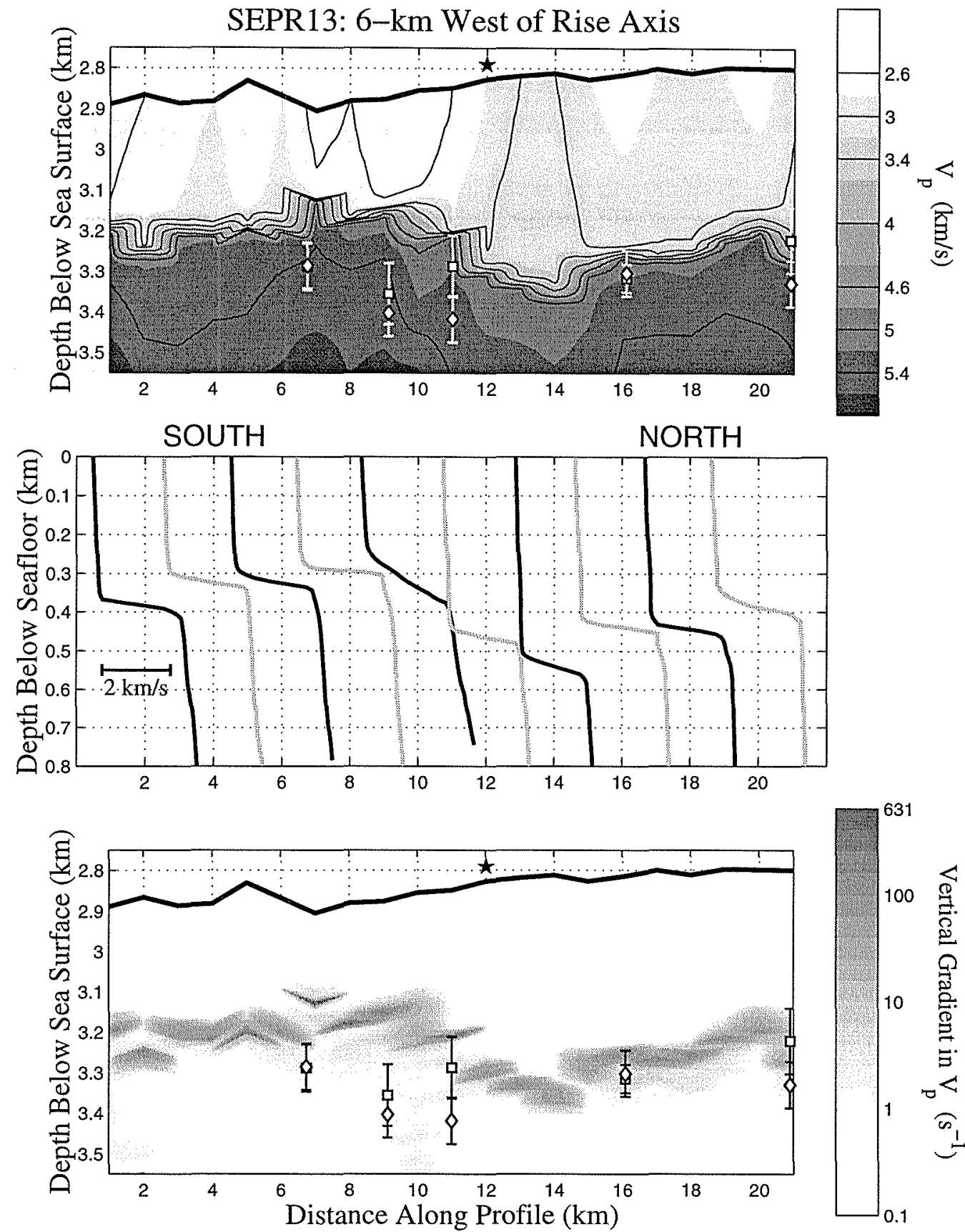

Figure 5-10: Same as Figure 5-8, except for the profile $6 \mathrm{~km}$ west of rise axis: SEPR13. The profile corresponds to 85 -ky-old crust and is plotted to $\sim 800$ meters depth. The star marks the location of the supergather shown in Figure 5-2a. Squares and diamonds in the upper and lower panel represent the off-axis picks for the base of layer 2A from Carbotte et al. [1997] and Hooft et al. [1997a], respectively. The transition zone is deeper in this profile than in both the along-axis (Figure 5-4) and $3 \mathrm{~km}$ off-axis (Figs. 5-6 \& 5-8) profiles. 
a) SEPR13 Supergather 12: Trace Comparison with Best Model (Unshifted) [ $\phi=23.523]$
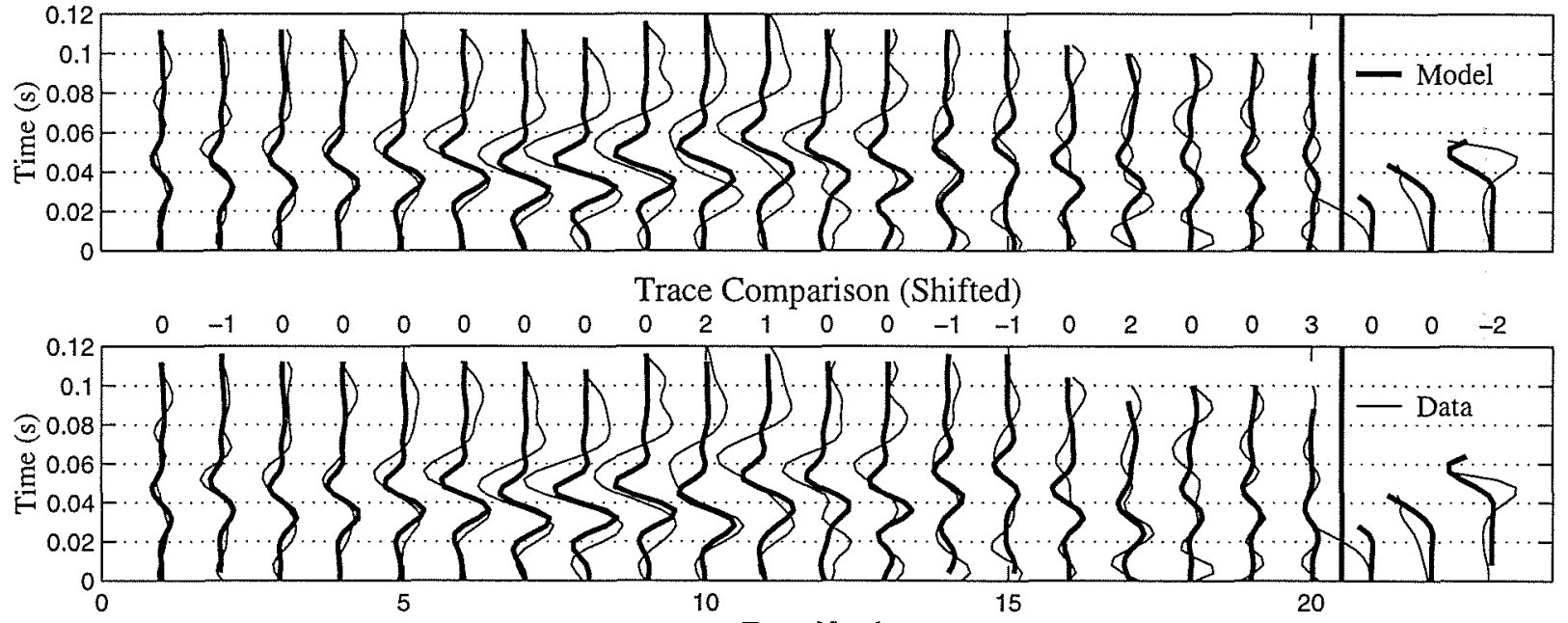

b) Mean Final Model Travel Times

Trace Number

c) Mean Final and Best Model
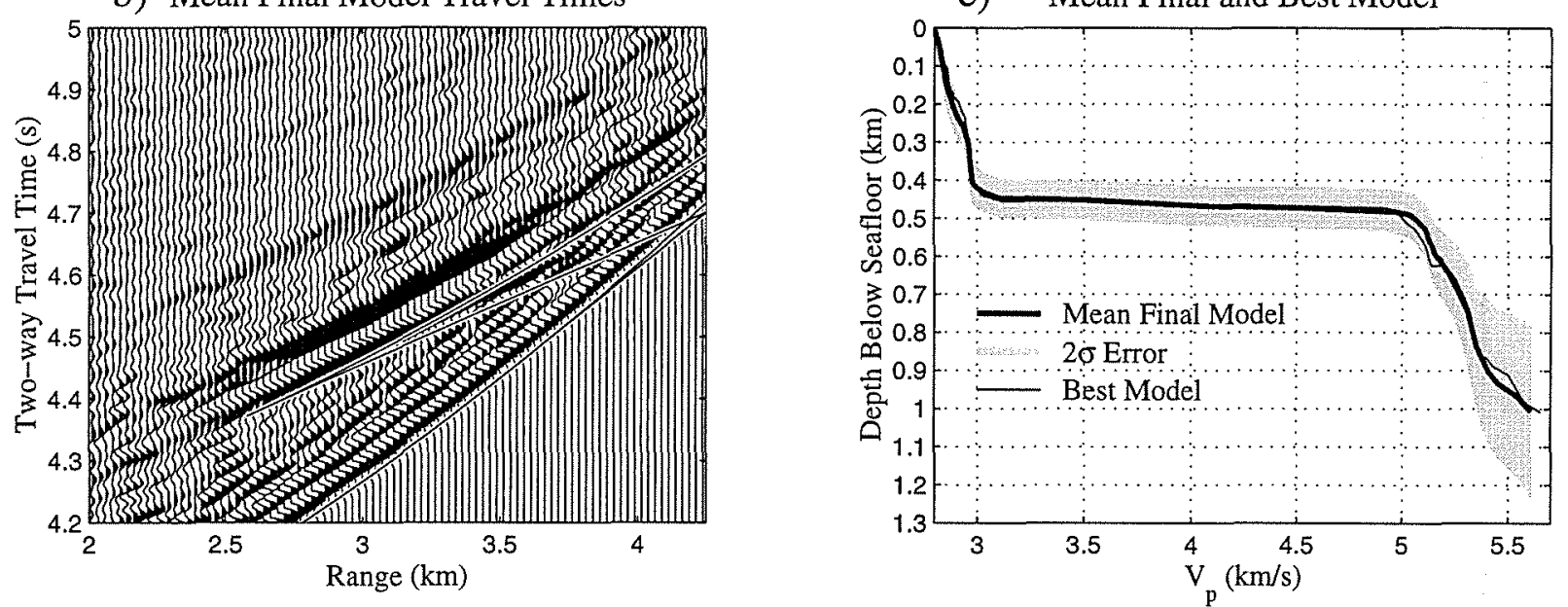

Figure 5-11: Same as Figure 5-9, except for the $6 \mathrm{~km}$ off-axis supergather 12, shown in Figure 5-2a and marked by a star in Figures 5-1 and 5-10. 

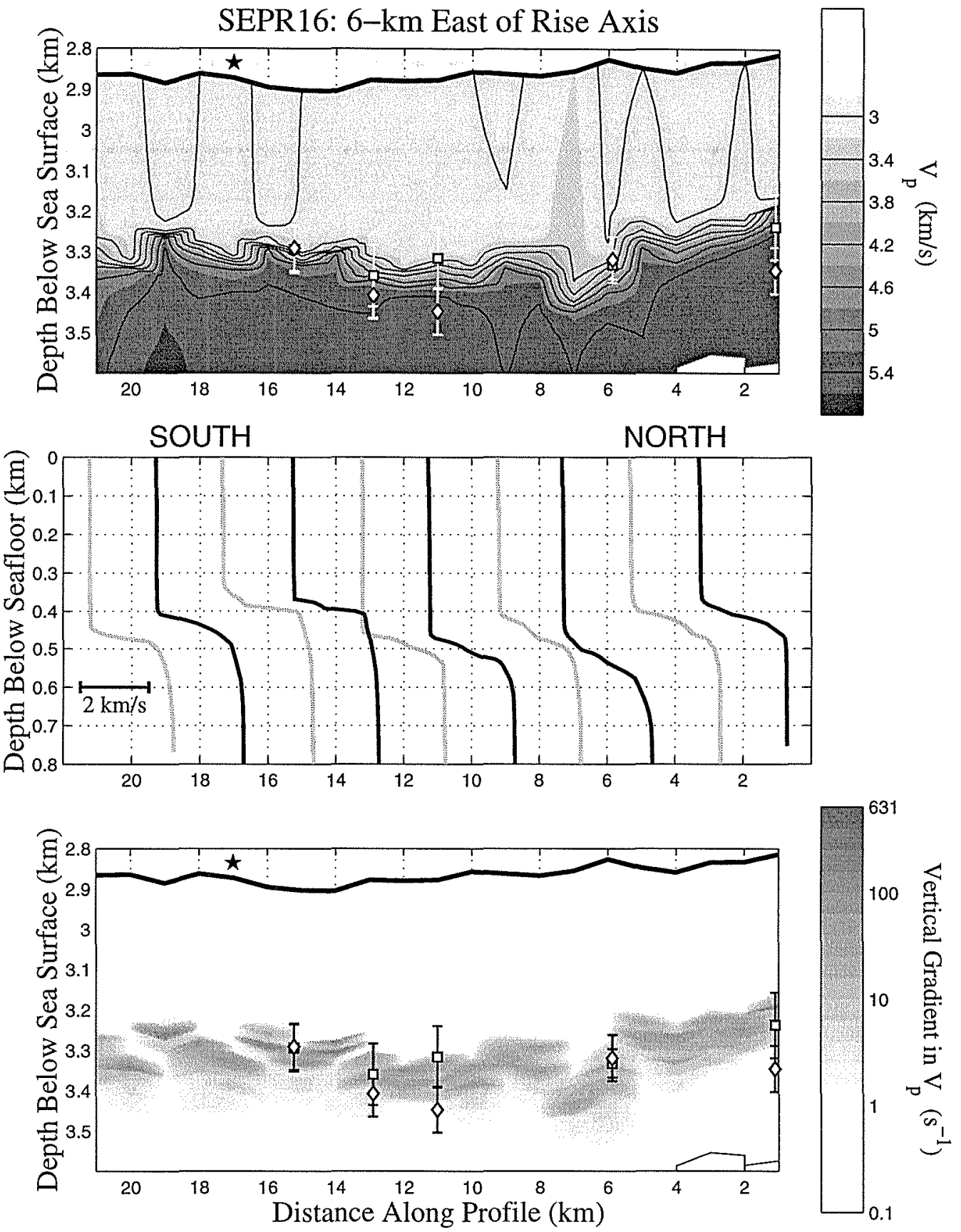

Figure 5-12: Same as Figure 5-10, except for the profile $6 \mathrm{~km}$ East of rise axis: SEPR16. The star marks the location of the supergather shown in Figure 5-2e. 
a) SEPR16 Supergather 17: Trace Comparison with Best Model (Unshifted) [ $\phi=22.097]$

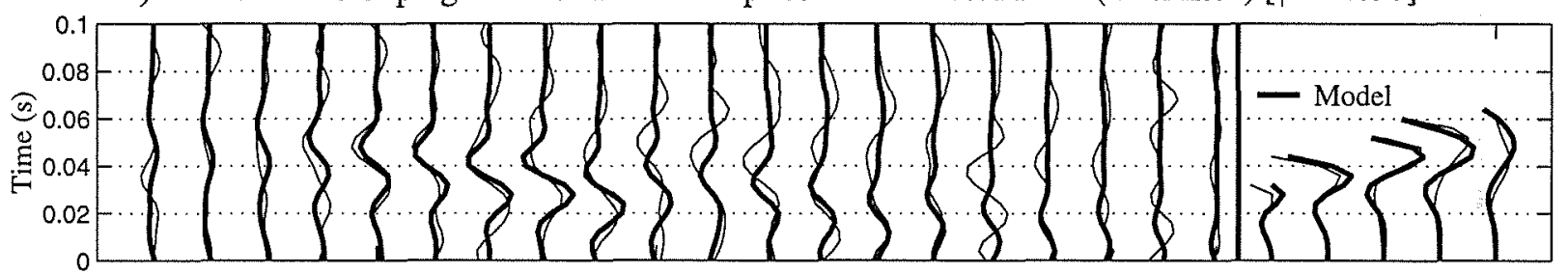

Trace Comparison (Shifted)

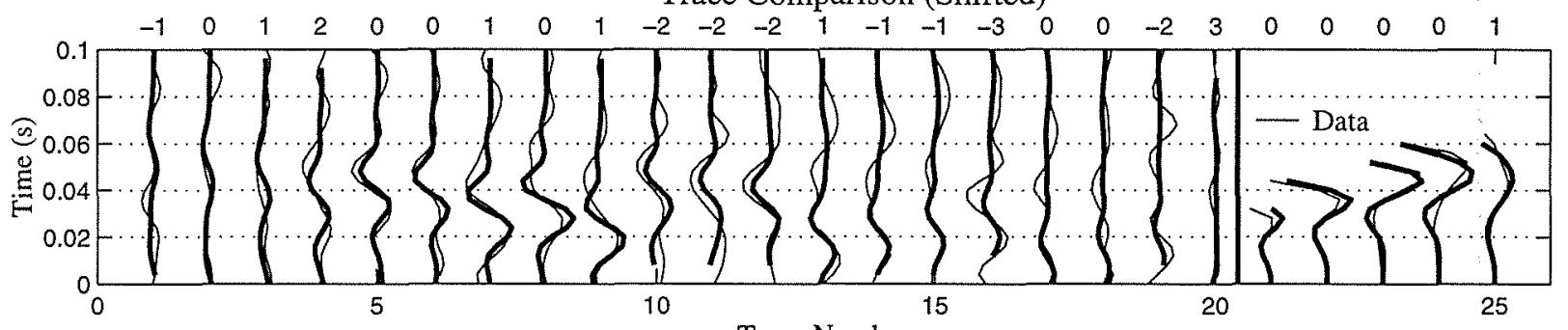

b) Mean Final Model Travel Times

Trace Number

c) Mean Final and Best Model
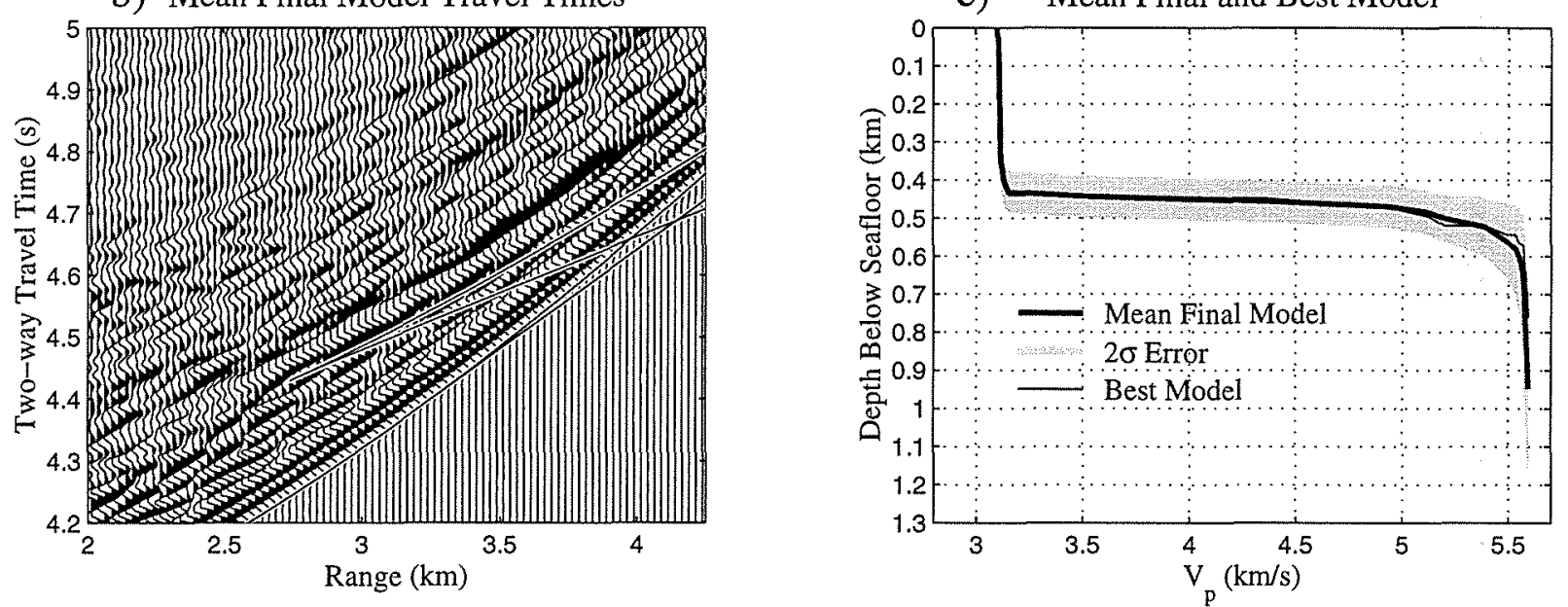

Figure 5-13: Same as Figure 5-11, except for the $6 \mathrm{~km}$ off-axis supergather 17, shown in Figure 5-2e and marked by a star in Figures 5-1 and 5-12. 

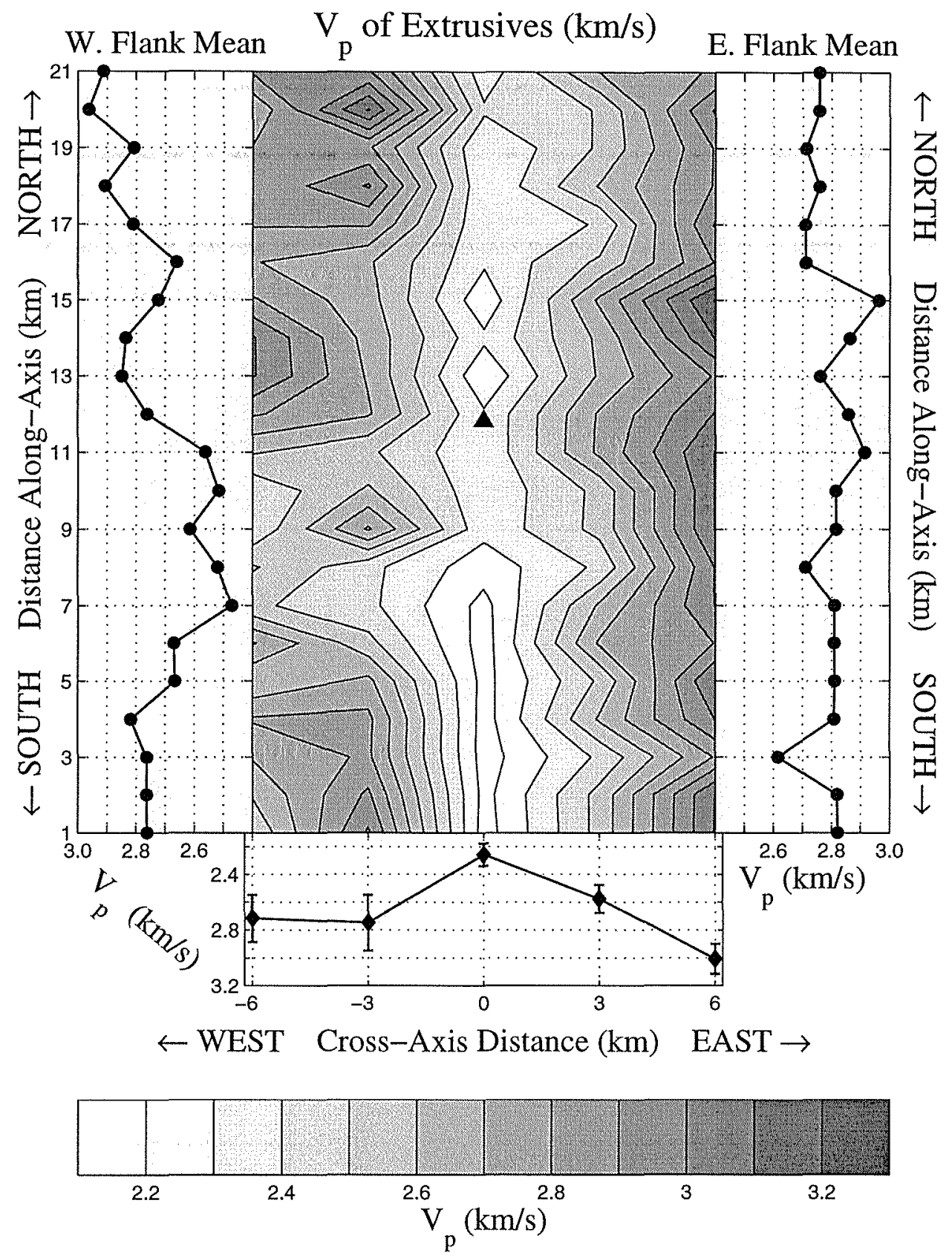

Figure 5-14: Contoured map of the mean velocity in the upper 50 meters of extrusive crust. A triangle on the rise axis marks where the axial magma chamber reflector reaches its shallowest point. The average velocity and standard deviation of each profile is shown in the lower panel to highlight the increase in velocity with age, although the two profiles on the western flank are essentially identical. The mean extrusive velocity on the flanks of the rise axis are shown in the side panels. Each panel is the average of the two corresponding off-axis profiles. Note the increase in velocities to the north along the rise axis, and the lack of such a trend off-axis. 
corridor of low velocities (between the 5 and $11 \mathrm{~km}$ mark) to the west. The higher variability along the western flank is reflected in the standard deviation assigned to the average of the two contributing profiles.

Layer $2 \mathrm{~A}$ thickness is difficult to measure by hand, since the abruptness and internal character of the transition from low to high velocities varies considerably. For purposes of consistency, we define layer $2 \mathrm{~A}$ as the depth interval containing material slower than $4 \mathrm{~km} / \mathrm{s}$. Since the major velocity transition at every location includes this value, our definition guarantees that at least part of the transition is sampled, and that our measure is linked to, albeit thinner than, the conventional layer $2 \mathrm{~A}$ definition. Layer 2A thickness is contoured in Figure 5-15. As with extrusive velocity, the off-axis thickening of layer $2 \mathrm{~A}$ is evident: $90 \mathrm{~m}$ on-axis, $320-390$ meters $3 \mathrm{~km}$ off-axis, and 380-440 meters $6 \mathrm{~km}$ off-axis. This represents a $250-350 \%$ increase in thickness within $3 \mathrm{~km}$ of the rise axis and continued thickening off-axis to the east. The along-axis and rise-parallel trends in thickness are different from those in $V_{p}$ (Fig. 5-14). Not only is layer $2 \mathrm{~A}$ extremely uniform on-axis, but off-axis it noticeably increases in thickness to the north. This occurs gradually along the eastern flank, and is step-like to the west. The sudden transition along the western flank occurs at the $11 \mathrm{~km}$ mark and may be related, perhaps coincidentally, to the proximity of the AMC at its shallowest point (triangle in Figs. 5-14 \& 5-15) [Detrick et al., 1993; Mutter et al., 1995].

\subsection{Discussion}

This study represents the first detailed velocity analysis performed on single-ship multichannel data. It is therefore helpful to gauge the usefulness of our velocity analysis technique by comparing these results to those from more conventional acquisition and processing methods. This comparison will allow us to interpret our inversion results more confidently in terms of construction and modification of upper oceanic crust. 

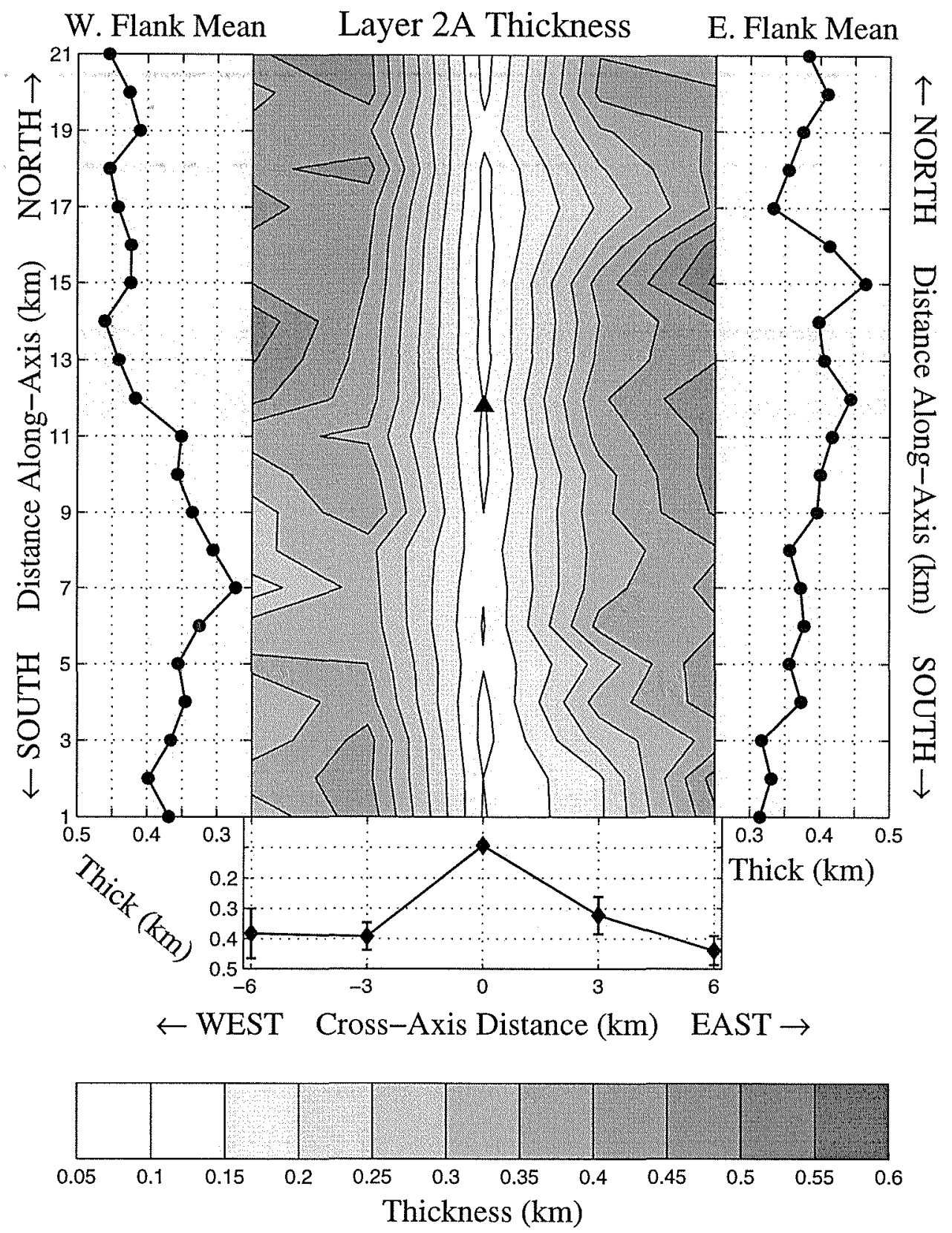

Figure 5-15: Contoured map of layer 2A thickness. Panels are arranged as in Figure 514. Extrusive thickness is defined as the depth to the $4 \mathrm{~km} / \mathrm{s}$ velocity contour. Note the extremely uniform along-axis thickness, and its increase with age. Also note the transition to a thicker extrusive section north of the AMC depth minimum (triangle). 


\subsubsection{Comparison with Previous Results and Methods}

A thin, surficial low-velocity layer on the order of $100 \mathrm{~m}$ or less is commonly found along the crest of the East Pacific Rise, but a double-step transition to layer $2 \mathrm{~B}$ velocities is not (Fig. 5-16). Expanding spread profile data at $9^{\circ} \mathrm{N}$ reveal a major jump in velocities $100 \mathrm{~m}$ below the seafloor, underlain by $100-150 \mathrm{~m}$ of low-gradient material and a second, smaller velocity step [Vera et al., 1990]. A detailed on-bottom survey in the same area confirms this pattern, but assigns a shallower depth $(<60$ m) to the upper transition [Christeson et al., 1994a]. Inversions of wide-aperture profile and ESP data at $13^{\circ} \mathrm{N}$ also yield a shallow low-velocity layer $(125-130 \mathrm{~m})$, but fail to detect a double-step structure [Harding et al., 1989; Kappus et al., 1995]. They predict a single, 150 to 200 -m-thick gradient transition, which places the base of layer $2 \mathrm{~A}$ at a depth comparable to other locations. The analysis of an ESP at $17^{\circ} 09^{\prime S}$ [Detrick et al., 1993], $20 \mathrm{~km}$ north of our study area, resolves an on-axis structure that is strikingly similar to our results. Although the depths to the two step-like transitions are slightly mismatched, the velocities encompassed by each are nearly identical. The comparison of these studies suggests that the rapid increase in velocities within $100-150 \mathrm{~m}$ of the seafloor is ubiquitous along the $\mathrm{EPR}$, but that the step-like lower transition is a less common, second-order feature. The similarity of our results with those of Detrick et al. [1993] indicates that the double-step transition to layer $2 \mathrm{~B}$ velocities is present, but possibly intermittent, along the rise crest between $17^{\circ}$ and $17^{\circ} 30^{\prime} \mathrm{S}$.

The on-bottom refraction results of Christeson et al. [1994a] show an off-axis deepening and strengthening of the double-step structure within the upper $500 \mathrm{~m}$ of crust. However, other studies on the northern EPR fail to confirm this result, including that of Vera et al. [1990], which was conducted in the same area. Our results also resolve only a single gradient transition off-axis, in agreement with the results of Vera et al. [1990] and Harding et al. [1989]. Other than these studies, no other upper crustal velocity analysis has been performed along the ridge flanks of the 

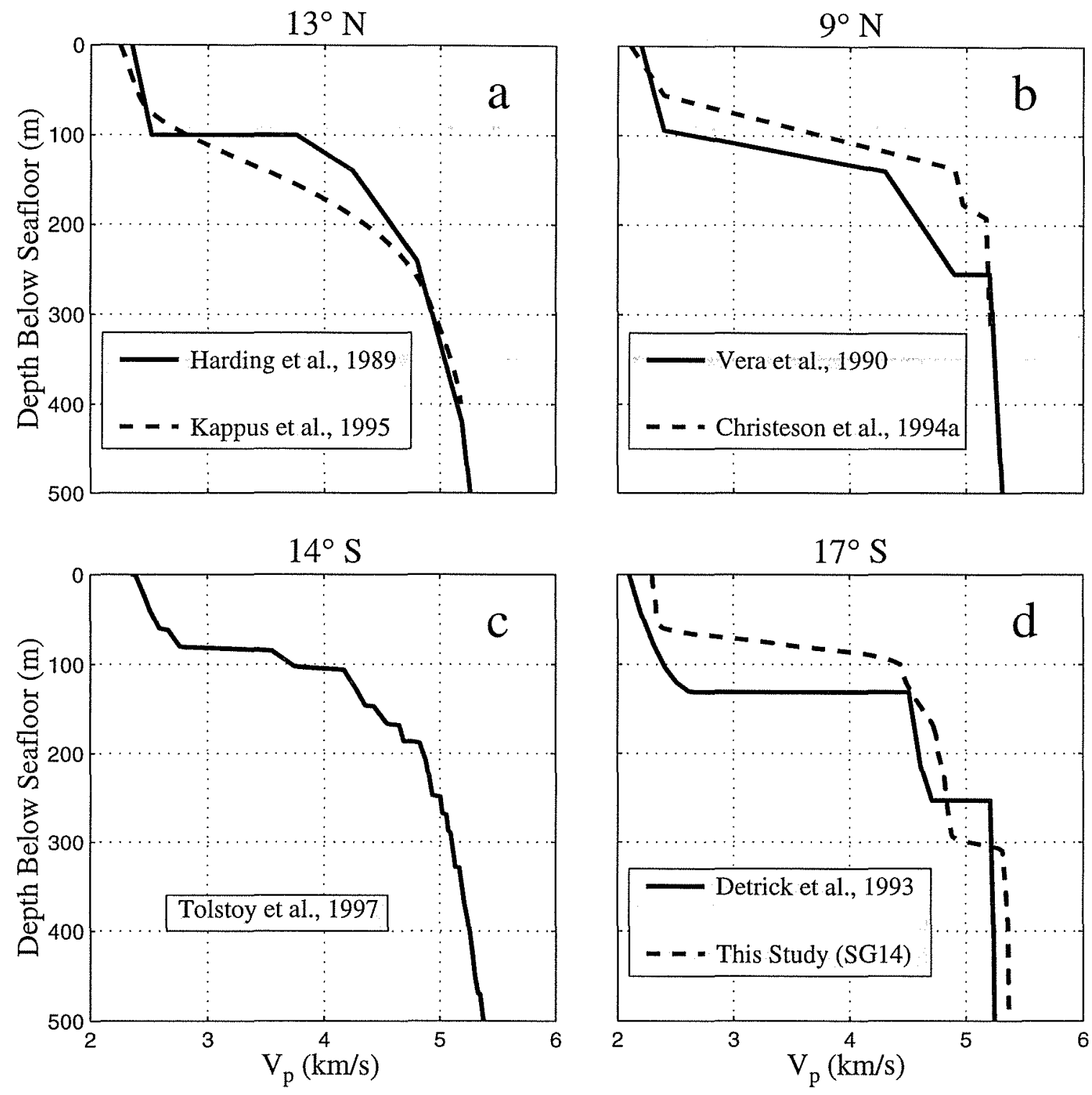

Figure 5-16: Velocity analysis results from four locations along the East Pacific Rise: a) ESP [Harding et al., 1989] and WAP data [Kappus et al., 1995] at $13^{\circ} \mathrm{N}$, b) ESP [Vera et al., 1990] and on-bottom data [Christeson et al., 1994a] at $9^{\circ} \mathrm{N}, \mathrm{c}$ ) WAP data [Tolstoy et al., 1997] at $14^{\circ} \mathrm{S}$, and d) ESP data [Detrick et al., 1993] at $17^{\circ} \mathrm{S}$. The representative on-axis velocity function from this study is included in panel d) for comparison. 
southern EPR. Clearly, additional information is needed to unambiguously establish the fine-scale velocity structure of crust on the flanks of the EPR.

Due to the predominance of reflected energy over refracted arrivals at small ranges, single-ship multichannel data have never been the subject of detailed velocity analyses. A more typical approach has been to create a cross-sectional image of subsurface reflectivity by collapsing each gather along a profile to its vertical-incidence equivalent. Previous authors have applied conventional CDP processing techniques to this data set in order to image and identify the base of layer 2A [Hooft et al., 1997a; Carbotte et al., 1997]. Hooft et al. [1997a], analyzing both cross- and along-axis profiles, found layer $2 \mathrm{~A}$ to thicken from an average of $230 \mathrm{~m}$ on-axis to $\sim 500 \mathrm{~m}$ outside the $2 \mathrm{~A}$ accumulation zone. In contrast, Carbotte et al. [1997] found that it thickened from 140 to $450 \mathrm{~m}$ on average along the same cross-axis lines- $40 \%$ and $10 \%$ thinner than the former values, respectively (Figs. 5-4, 5-10 \& 5-12). The difference of nearly 100 $\mathrm{m}$ between the two on-axis estimates falls well outside the error bounds associated with depth conversion, and can only be explained by the presence of more than one distinct horizon. In fact, the values of Hooft et al. [1997a] match both the depth and variation pattern of the lower transition zone found in this study, while those of Carbotte et al. [1997] correspond more closely to the upper gradient (Fig. 5-4). This provides further evidence for a double-step transition to layer $2 \mathrm{~B}$ velocities in this area. Within the resolution of their analysis techniques, these studies agree on the off-axis thickness of layer $2 \mathrm{~A}$ and, together, completely span the depth interval over which the velocity transition occurs (Figs. 5-10 \& 5-12).

To further compare our waveform inversion results to those from conventional CDP processing, we created stacked seismic images of on-axis line SEPR17 and off-axis line SEPR16 (Figs. $5-17 \& 5-18$ ). Moveout velocities between $1.5 \mathrm{~km} / \mathrm{s}$ (seawater) and $2.2 \mathrm{~km} / \mathrm{s}$ (typical of the magma sill) were tested on constant velocity stacks of the data, and a coarse stacking velocity model was generated every $250 \mathrm{~m}$. The seismic profiles were then constructed with the velocity field from this analysis. Ranges be- 

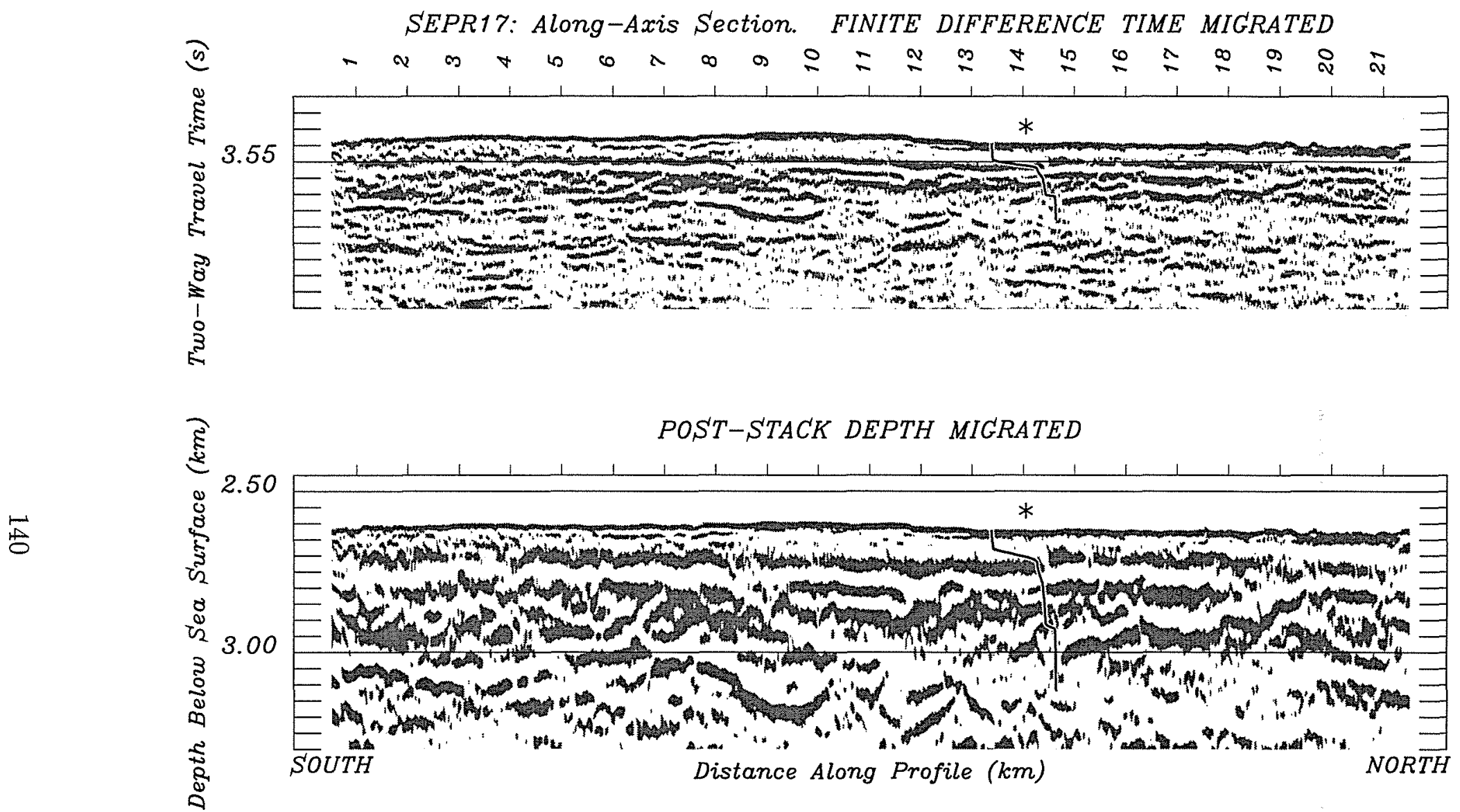

Figure 5-17: Stacked seismic section of the along-axis profile, SEPR17, time migrated with the $45^{\circ}$ finite difference algorithm [Brysk, 1983] (top), and depth migrated with the split-step algorithm of Stoffa et al. [1990] (bottom). Negative amplitudes are white, and positives are dark. Energy above the seafloor is muted. The site examined in Figure 5-5 is marked by a star, beneath which the GA-derived velocity model is drawn. Note the excellent agreement between the velocity and reflectivity data regarding the depth of the two seismic horizons. Energy from these horizons is refracted to varying degrees, and is therefore phase-shifted with respect to the seafloor event. In some areas, the lower event is diffuse, and difficult to identify. 


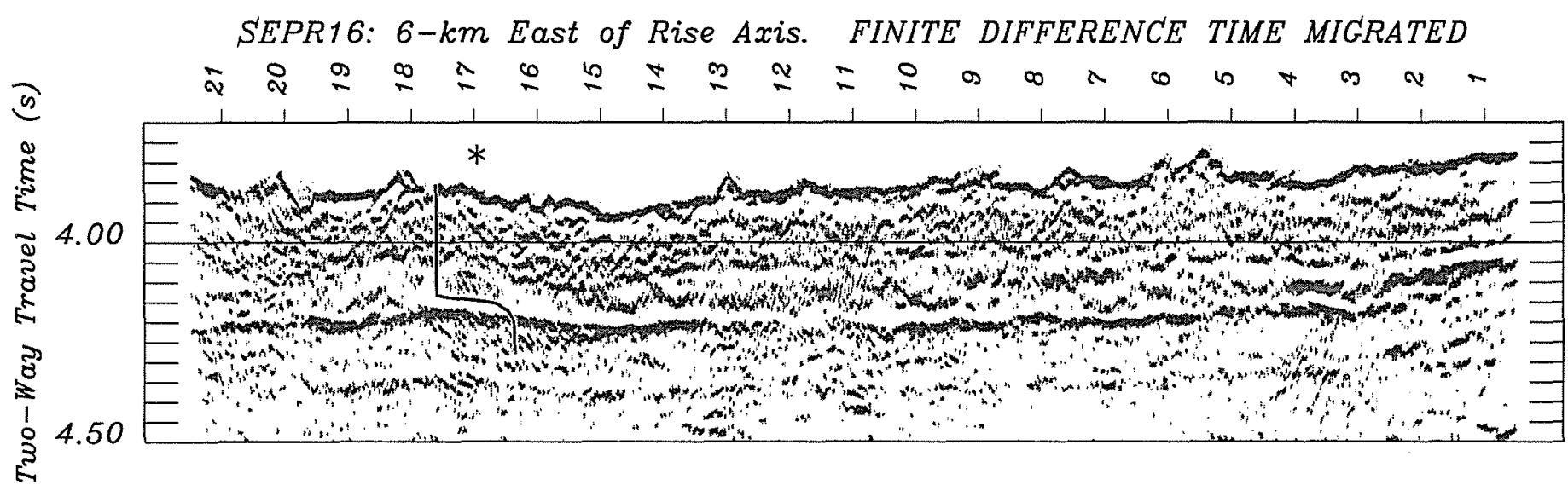

\section{POST-STACK DEPTH MIGRATED}

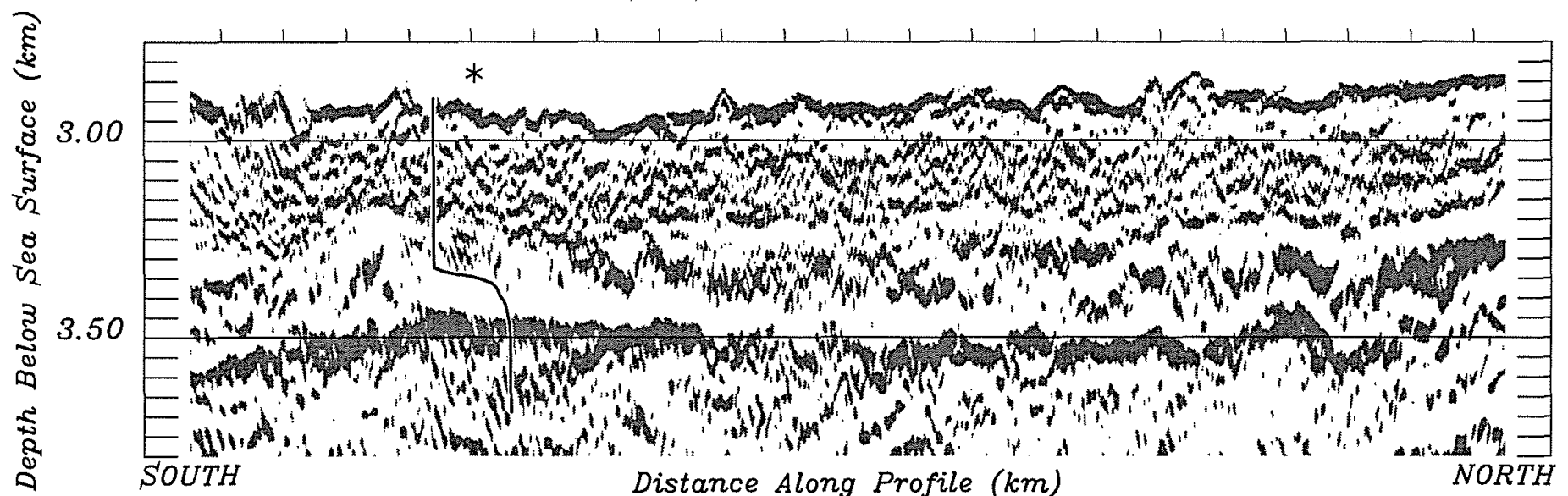

Figure 5-18: Same as Figure 5-17, except for the profile $6 \mathrm{~km}$ east of rise axis: SEPR16. CDP processing produces a single, deeper horizon that spans a considerable depth interval in some regions. The mean final model of Figure 5-5 is also plotted. Note the excellent agreement between the predicted depth of the velocity transition and the onset of the layer $2 \mathrm{~A}$ horizon. 
yond $3.2 \mathrm{~km}$ were not included in the final stacks, because the nonhyperbolic (i.e., non-reflective) moveout of refractions from the gradient at the base of layer $2 \mathrm{~A}$ forces the stacking process to distort this event at far offsets [Harding et al., 1993; Vera and Diebold, 1994]. As a final step, the profiles were either time migrated with a $45^{\circ}$ finite difference algorithm [Claerbout, 1976; Brysk, 1983] or depth migrated with the split-step algorithm of Stoffa et al. [1990] (Figs. 5-17 \& 5-18). The finite difference algorithm was chosen for its ability to handle steep dips and two-dimensional velocity variations. In addition to focusing energy in strongly varying media, the depth migration places horizons at their true subsurface position, but in doing so, requires an accurate representation of the velocity field. The velocity functions generated by the GA are ideal for that purpose (Figs. 5-4 \& 5-12, top), and were therefore supplied to the split-step algorithm.

On-axis, two distinct horizons are imaged below the seafloor (Fig. 5-17). They correspond to the upper and lower transition zones predicted by the waveform inversion (Fig. 5-4). We define the location of these subsurface events as the transition to positive (dark) amplitudes, since refracted energy is phase-shifted with respect to the negative-onset seafloor reflection. The upper horizon is optimally stacked 0.075 to 0.1 $\mathrm{s}$ below the seafloor with a moveout velocity of $1525 \mathrm{~m} / \mathrm{s}$. Applying the GA-derived velocity field, this corresponds to a thickness of $\sim 100 \mathrm{~m}$. The lower horizon is most clearly focused with moveout velocities near $1600 \mathrm{~m} / \mathrm{s}$, and is located at a variable basement depth of 250-425 m, with regions of diffuse reflectivity at the center and northern end of the profile. Whereas the upper horizon in the stack sections exactly matches that predicted by the GA, the lower one is imaged too deeply in the south. Only in the north-central portion (14-18 km mark), where the deeper velocity transition is step-like, is this horizon shallow enough to match the depths predicted by the GA.

The source of this mismatch lies in the assumptions made during the stacking procedure and in the sensitivity of the waveform inversion to velocities between the 
two transitions. Stacking refractions along hyperbolic moveout curves not only distorts the event [Harding et al., 1993; Vera and Diebold, 1994], but also places it too deeply at zero offset [Harding et al., 1993; Christeson et al., 1996]. The shallower the gradient, and larger its depth extent, the more stacking will overshoot its true location. Treating the 2A transition as a single gradient, Harding et al. [1993] showed that energy from its base is imaged too deeply by up to $80 \mathrm{~m}$, depending on the average velocity used. The common practice of muting the farthest offsets, where the assumption of hyperbolic moveout breaks down, further deepens the rendering of gradient horizons by including energy only near their caustic (i.e., base). Carbotte et al. [1997] estimate that the combined uncertainties associated with CDP processing may produce layer $2 \mathrm{~A}$ thickness errors on the order of 50-200 $\mathrm{m}$. This is quite extreme, especially when dealing with depth estimates of only 100-500 meters. The GA-derived velocity functions are also not free of uncertainty. Much of the energy turning immediately above and within the lower transition zone is partially masked by the seafloor event in the data gathers (e.g., Fig. 5-5b). Since the velocities across this depth interval are less constrained by the data, the uncertainty estimates for this region are larger. The fact that the deeper horizon stacks best at a velocity lower than the $1675 \mathrm{~m} / \mathrm{s}$ derived from GA results indicates that the average velocity between the two transitions may be up to $1 \mathrm{~km} / \mathrm{s}$ lower than the inversion results suggest.

Off-axis, we image a single, broad event (Fig. 5-19) that is best focused by a stacking velocity that varies between 1600 and $1700 \mathrm{~m} / \mathrm{s}$. Its average depth of $\sim 450$ $\mathrm{m}$ matches that given by the GA (Table 5.1). In fact a slight thickening of layer $2 \mathrm{~A}$ in the north-central portion of this profile is predicted by both methods. In summary, the successful application of our velocities to the depth conversion of CDP data show that enough velocity information exists in single-ship multichannel data without turning to outside sources (i.e., refraction of two-ship experiments).

A comparison of the cross-axis reflectivity profile, SEPR27 [G. Kent, unpublished data], with the velocity functions nearest to it, shows that, within the error bounds 


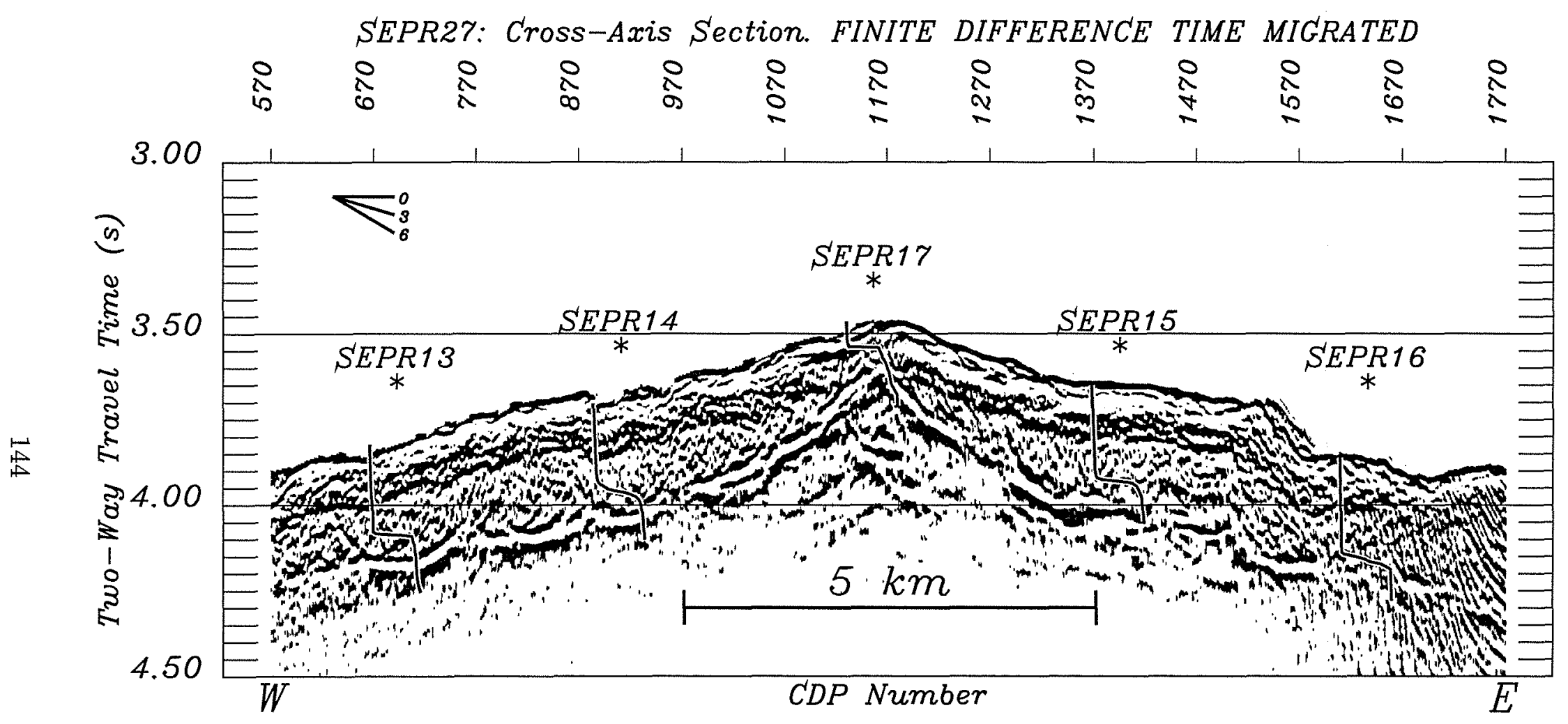

Figure 5-19: Stacked, time migrated seismic section of cross-axis profile SEPR27, shown in Figure 5-1. The profile was provided by G. Kent (unpublished data). Velocity models corresponding to the midpoint (11-km mark) of all five on- and along-axis profiles are plotted at their point of intersection with this line. The layer $2 \mathrm{~A}$ horizon correlates well with the base of the velocity gradient off-axis and the upper transition on-axis. A dip diagram illustrates seafloor slopes of $0^{\circ}, 3^{\circ}$ and $6^{\circ}$. 
discussed above, the layer $2 \mathrm{~A}$ thickening profile matches the transition zone depths predicted by this study (Fig. 5-19). Careful processing of SEPR27 reveals that the layer $2 \mathrm{~A}$ horizon is a continuous event that shoals to within $0.12 \mathrm{~s}(<140 \mathrm{~m})$ of the seafloor at the rise crest. This on-axis depth corresponds more closely to the upper velocity transition, which is the sharper and more continuous of the two gradient zones detected at the rise crest (Fig. 5-4). In linking the off-axis layer $2 \mathrm{~A}$ event to the shallow rise crest transition zone, we argue that the layer $2 \mathrm{~A}$ thickness map of Figure 5-15 correctly represents the cross-axis geometry of a distinct geological horizon that deepens by $300-400 \%$ within $1-4 \mathrm{~km}$ of the rise axis. Since previous seismic estimates of on-axis layer $2 \mathrm{~A}$ thickness have typically been larger than presented here [e.g., Kent et al., 1994; Hooft et al., 1997a; Carbotte et al., 1997], our thickening profile represents a significant revision to the off-axis doubling previously attributed to layer $2 \mathrm{~A}$. The excellent correlation between our results and the reflection profiles, in general, demonstrates the ability of the GA-based inversion to extract detailed velocity information from single-ship data, and establishes these velocity models as an accurate representation of the upper crust from which geological interpretations can be made.

\subsubsection{Aging of Extrusive Crust}

The off-axis increase in compressional wave velocities of the extrusive crust is well established in our study area (Fig. 5-14 \& Table 5.1). Since Houtz and Ewing [1976] first documented an age-dependent increase in the velocity of the uppermost crust, several studies have confirmed this relationship. In the Pacific alone, these studies include conventional refraction [Spudich and Orcutt, 1980; Purdy, 1982; Bratt and Purdy, 1984; Bratt and Solomon, 1984; McClain and Atallah, 1985; Bee and Bibee, 1989; Grevemeyer and Weigel, 1997; Grevemeyer et al., 1997], borehole [Stephen and Harding, 1983; Little and Stephen, 1985; Duennebier et al., 1987; Whitmarsh et al., 1987; Collins et al., 1989], single-ship [Detrick et al., 1993; Tolstoy et al., 1997] 
and two-ship multichannel [Harding et al., 1989; Vera et al., 1990], and on-bottom seismics [Christeson et al., 1994a]. Sensitivity to velocities immediately beneath the seafloor varies between methods. For instance, the large trace spacing of conventional refraction studies limits how shallow the layer $2 \mathrm{~A}$ prograde event can be accurately tracked. The short trace spacing of multichannel studies allows energy turning just below the seafloor to be precisely located, but only in areas where extrusive velocities are high enough $(>3 \mathrm{~km} / \mathrm{s})$ to place the $2 \mathrm{~A}$ prograde branch within the streamer aperture ( $>50$-kyr-old crust in this case). Otherwise, as with the three youngest profiles in this study, the retrograde branch is used to constrain surficial velocities, since it contains information integrated through the entire upper section.

Velocities in the upper crust are predominantly controlled by porosity. As a result, the off-axis increase in $V_{p}$ is commonly attributed to the precipitation of hydrothermal minerals in small-scale pores and the collapse of voids in the form of lava pillows and tubes. The reduction of large-scale porosity is a function of overburden pressure, rock strength and tectonic forces acting within the basaltic pile. The extent that hydrothermal processes contribute to the modification of oceanic crust is largely governed by permeability and proximity to a heat source. At the rise crest, the combination of a shallow melt supply zone and highly disrupted extrusives provides an ideal environment for hydrothermal processes to permeate the volcanic section and decrease its bulk porosity. As the increasingly impermeable basaltic crust is transported away from its heat source, an accumulating sediment cover reinforces the transition from a vigorous, openly convecting system to a weak, closed system with little water-rock interaction and, hence, porosity change. The crustal age at which this transition occurs is not well constrained, and is likely to vary somewhat between ocean basins and from one volcanic flow to another.

The rapid increase in extrusive velocities recorded within $6 \mathrm{~km}$ of the rise axis indicates that crust less than $\sim 100 \mathrm{kyr}$ old is permeable and unsedimented enough to allow the hydrothermal infilling of small-scale voids to remain a vigorous process. A 
comparison between our results with seismic velocities for other locations along the flanks of the EPR reveals that the increase we record in the $17^{\circ} 20^{\prime} \mathrm{S}$ area is faster than for the EPR as a whole (Fig. 5-20). This may be linked to the anomalously shallow magma sill and robust cross-sectional shape of the rise crest-both indicators of a high magma (i.e., heat) budget. Such a shallow heat source and pervasive volcanic activity would drive a vigorous hydrothermal system that would, in turn, substantially decrease porosity in the near-ridge environment.

The difference between the east and west flank in both layer $2 \mathrm{~A}$ velocity and thickness (Figs. 5-14 \& 5-15) may be symptomatic of asymmetric spreading. Based on the location of the Brunhes/Matuyama magnetic polarity reversal (0.78 Ma), Naar and Hey [1989] and Cormier and Macdonald [1994] show that spreading asymmetry in this region is pronounced (up to $40 \%$ ) and highly variable, with the fast direction abruptly switching to the western flank north of our study area. Since the formation (e.g., Fig. 5-19) and significant maturation of layer $2 \mathrm{~A}$ is achieved well within the Brunhes epoch (Fig. 5-20), we are unable to resolve whether the skewed cross-axis trends in layer $2 \mathrm{~A}$ are the result of current asymmetric spreading. A cross-axis bias in the character of layer $2 \mathrm{~A}$ may simply be the result of short-term (i.e., $<100 \mathrm{kyr}$ ) variability in volcanic deposition, independent of spreading asymmetry.

Non-systematic kilometer-scale velocity variations may reflect local differences in crack formation or changes in volcanic morphology - for example, the corridor of low velocities on the western flank of the rise crest (Fig. 5-14). Laboratory [Gregg and Fink, 1995] and numerous field observations (e.g., Luyendyk and Macdonald [1985]; Fox et al. [1987]) document a variety of flow morphologies produced at the rise crest, which are likely to vary in their ability to impede fluid flow into the volcanic substructure [Pezard, 1990].

Recently, Grevemeyer and Weigel [1996] and Carlson [1998] compiled the most comprehensive set of compressional wave velocities for acoustic basement since Houtz and Ewing [1976]. Grevemeyer and Weigel [1996] approximate the relationship be- 


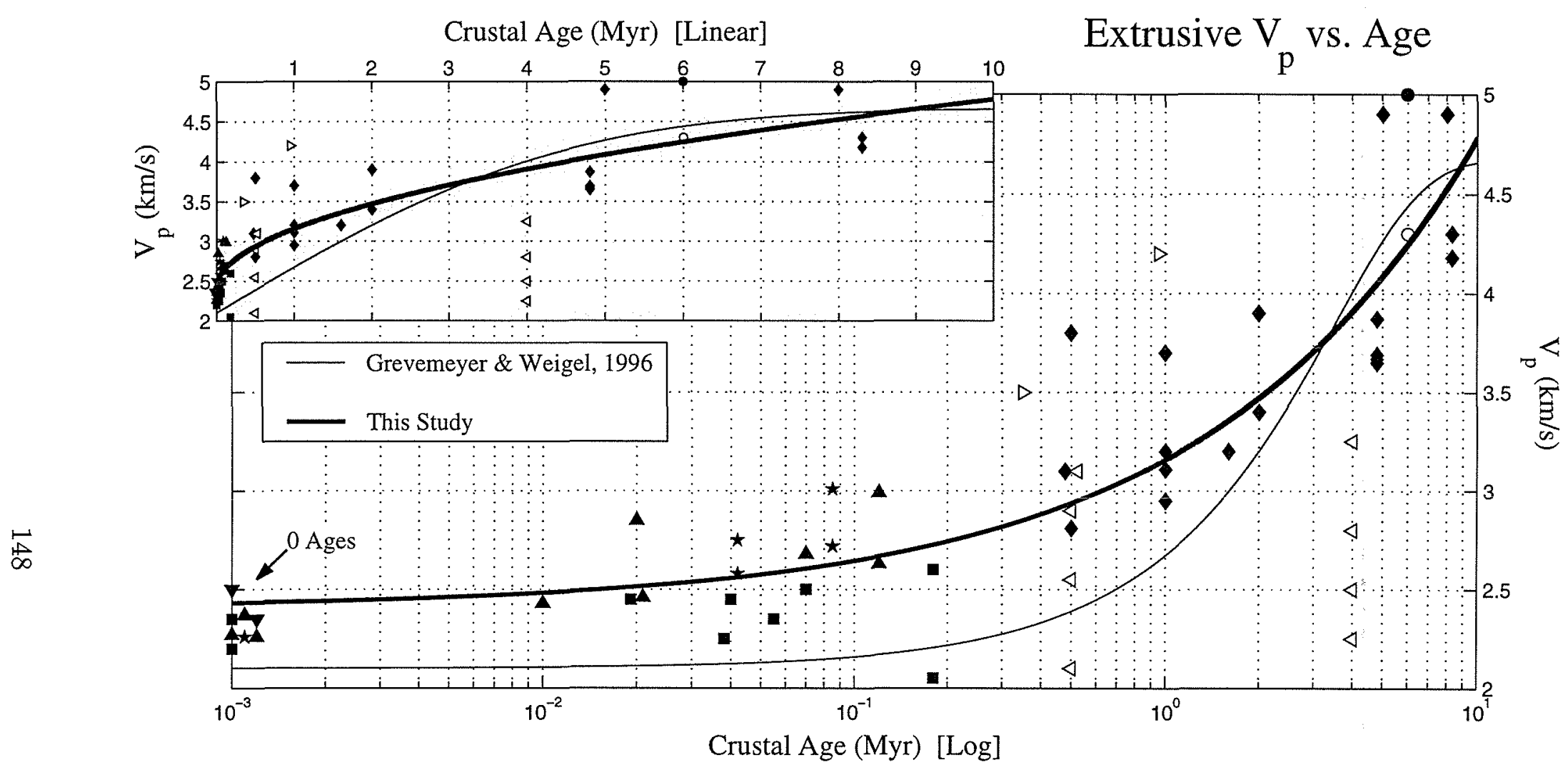

Figure 5-20: Compressional wave velocity in the extrusive section as a function of crustal age. Included is data from ROSE

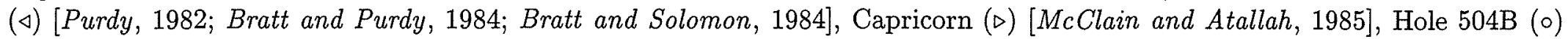

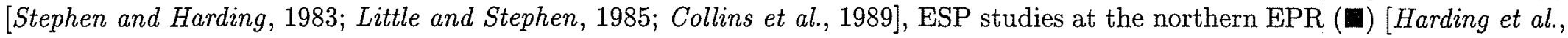
1989; Vera et al., 1990], multichannel studies at the southern EPR ( $\mathbf{\nabla})$ [Detrick et al., 1993; Tolstoy et al., 1997], EXCO ( $)$ [Grevemeyer and Weigel, 1997; Grevemeyer et al., 1997], NOBEL ( $\mathbf{\Delta}$ [Christeson et al., 1994a], and this study ( $\star$ ). The best fitting curve from Grevemeyer and Weigel [1996] (thin) and this study (thick) are shown. Results older than a decade (i.e., ROSE, Capricorn and early 504B) were not used in the least squares inversion and are marked by an unfilled symbol. The inset plots the data of the larger, log-scale graph as a linear function of age. 
tween layer $2 \mathrm{~A}$ velocity and plate age with a single error function. However, the leverage of data points for crust older than $100 \mathrm{Myr}$ results in a substantial misfit at young ages. In Figure 5-20, we add the results from this study to a similar compilation, but only for basement ages less than 10 Myr. A least squares inversion of these data yields a best fitting function defined by the square root of age:

$$
V_{p}(t)=V_{0}+\alpha t^{\frac{1}{2}} \quad V_{0}=2.41, \quad \alpha=0.75
$$

$V_{0}$ is the zero-age velocity and $\alpha$ is a constant. The $t^{\frac{1}{2}}$ approximation produces an excellent fit to the data, with a least squares misfit three times smaller than that of the best fitting error function. For ages greater than 10-20 Myr, however, velocities increase more slowly and are better modeled by the curve of Grevemeyer and Weigel [1996] or a simple exponential relation. This transition may mark the range of crustal ages at which the combination of crustal cooling and sediment cover causes hydrothermal circulation to become a closed system that reduces porosity more

slowly. The above empirical approximation is similar to those for seafloor subsidence and heat flow, which also switch from a square root of age relation to an exponential one. Considering that the reduction of porosity through hydrothermal processes is, in part, thermally controlled, the similarity may not be coincidental.

\subsubsection{Geological Interpretation}

It has been established that the most prominent seismic horizon in the uppermost crust of this area, and of fast-spreading ridges in general (Fig. 5-16), is associated with a dramatic velocity jump of over $2 \mathrm{~km} / \mathrm{s}$ (Fig. 5-16), and that the subseafloor depth of this event increases from $\sim 100 \mathrm{~m}$ at the rise crest to over $400 \mathrm{~m}$ along its flanks (Figs. 5-15 \& 5-19).

This feature has previously been interpreted in terms of a porosity horizon within the extrusive section. Such a lithology-independent horizon may exist as a lithostatic 
compaction boundary, below which cracks are mechanically sealed by the increased overburden pressure [Spudich and Orcutt, 1980; Vera et al., 1990], or as a tectonic fracture front that deepens off-axis [McClain et al., 1985]. However, the systematic relationship between confining pressure and depth should result in a velocity increase that is more gradual than we observe [Birch, 1960]. Similarly, there is no requirement that tectonic fracturing be localized within the upper extrusives rather than be pervasive. The tectonic deepening of a fracture front off-axis is also difficult to reconcile with seafloor observations, which show the onset of large-scale fracturing to occur outside the zone where layer 2A thickening begins [Bicknell et al., 1987; Edwards et al., 1991; Carbotte et al., 1997]. The layer 2A horizon may alternatively be hydrothermal or metamorphic in origin [Rohr et al., 1988; Vera et al., 1990; Wilkens et al., 1991]. The primary evidence in support of this argument comes from DSDP/ODP Hole 504B, where a transition in seismic velocity [Becker et al., 1988], acoustic attenuation [Wilcock et al., 1992], permeability [Anderson et al., 1985] and porosity [Becker et al., 1982] is recorded within the extrusive section at basement depth of 100-200 m. However, as Harding et al. [1993] and Christeson et al. [1994a] point out, this site is situated in crust that has matured considerably during its 6 Myr existence. Both argue that the base of layer $2 \mathrm{~A}$ represents a porosity boundary that is lithologically controlled near the rise crest, and shoals with crustal age as pore spaces within the extrusives are filled with alteration products. Petrographic analyses of recovered samples from Hole 504B detect a zeolite alteration front that separates the lower lavas, characterized by secondary mineral formation in veins and vugs, from the overlying "submarine weathering" zone of the upper lavas [Alt et al., 1986]. Porosity studies of the Troodos Ophiolite, thought to be late Cretaceous in origin, reveal a similar decrease in porosity $250 \mathrm{~m}$ below the paleoseafloor [Gillis and Sapp , 1997]. The shoaling of such a hydrothermally controlled porosity front may still be an active process at Hole 504B [e.g., Becker et al., 1989], and is consistent with global compilations of seismic refraction data, which show a systematic thinning of 
layer 2A with crustal age [Houtz, 1976; Houtz and Ewing, 1976]. A porosity boundary may also form within the extrusive section if the lower lavas, emplaced at the rise axis, exhibit a fundamentally different flow morphology than those deposited on its flanks, as argued by Harding et al. [1989]. Although thin $(<5 \mathrm{~m})$ sheet flows have been observed within the axial summit trough [Haymon et al., 1993], and pillow flows off-axis [Fornari et al., 1992; Perfit et al., 1992], the segregation of lava morphologies by rise axis proximity is not well established.

Possibly a more fundamental difference between on- and off-axis flows is their respective likelihood to be intruded by a future diking event, and hence be incorporated into the pillow/dike transition. Extrusives deposited on-axis, especially within the summit trough, and ultimately buried at the base of the volcanic pile, are likely to be riddled with low-porosity dike material, which would increase their bulk velocity with respect to the overlying lavas deposited outside the dike intrusion zone. We therefore believe that the major velocity transition in young upper oceanic crust is controlled by, and is coincident with, the downward transition from the high-porosity extrusive basalts to sheeted dikes, and that its cross-axis thickening profile is governed by the successive buildup of lavas on the flanks of the rise crest.

Numerical models that simulate the construction of the upper crust support a lithologic origin of the layer $2 \mathrm{~A}$ seismic event. By modeling the repeated deposition of lava flows, these studies successfully predict not only the structure observed at ophiolites [Dewey and Kidd, 1977; Kidd, 1977] and Icelandic rift zones [Pálmason, 1980], but also the off-axis thickening geometry of layer $2 \mathrm{~A}$ at fast spreading ridges [Hooft et al., 1996]. A rapid subsidence of the sheeted dikes beneath a thickening extrusive layer is the preferred interpretation of the shallow, high-velocity anomalies observed along the rise crest by tomographic studies [Toomey et al., 1990, 1994]. Direct observations of the pillow/dike transition have only been made in mature ( $\geq 1$ Myr old) or obducted ocean crust. These include drill-hole measurements (Hole 504B: $571 \mathrm{~m}$ [Becker et al., 1989], Hole 395A: >571 m [Shipboard Scientific Party, 1979]), 
submersible observations (Hess Deep: 100-200 [Francheteau et al., 1992], Vema fracture zone: 280-800 m [Auzende et al., 1989; Cannat et al., 1991], FAMOUS Area: $230 \mathrm{~m}$ [Francheteau et al., 1975]), and ophiolite studies (Samail, Oman: 750-1500 m [Alabaster et al., 1982]; Troodos, Cyprus: 1-1.5 km [Schmincke et al., 1983]). Because of the variability in these estimates, and the geological setting from which they come (e.g., slow spreading or highly tectonized environment), it is unreasonable to present any as a paradigm for young, fast spreading crust. However, as a whole, these estimates are consistent with the $350-550 \mathrm{~m}$ thicknesses that layer $2 \mathrm{~A}$ is observed to attain on the flanks of the EPR [e.g., Kent et al., 1994; Hooft et al., 1997a; Carbotte et al., 1997; this study].

Since lithologically controlled porosity variations are thought to dominate the seismic structure of young upper crust, our velocity functions may be a proxy for the depth distribution of low-porosity dikes $(5.4-5.8 \mathrm{~km} / \mathrm{s})$ within the high-porosity extrusives $(2.3-2.9 \mathrm{~km} / \mathrm{s})$. An important consequence of this interpretation is that the surficial low-velocity interval represents a layer of nearly pure extrusives, below which the dike fraction drastically increases. The existence of such a layer at the rise crest (Fig. 5-4) constrains the zone of dike emplacement to be quite narrow, since a wide dike distribution would permeate the entire near-axis volcanic section with high-velocity diabase.

To better constrain the extent of lava and dike emplacement in the $17^{\circ} 20^{\prime} \mathrm{S}$ area, we modify the crustal emplacement equations of Hooft et al. [1996], and apply them to our results (Table 5.1). In this method, a Gaussian probability (standard deviation, $\left.\sigma_{i}\right)$ is assigned to the spatial distribution of dike emplacement. A Rayleigh distribution (standard deviation, $\sigma_{e}$ ), the integral of a Gaussian, is assigned to the maximum distance of lava emplacement so as to form a Gaussian-shaped thickening profile at steady-state. Given geologically reasonable values for lava flow thickness $(t)$, dike width $(w)$, and probability of dike eruption $\left(P_{d}\right)$, the emplacement model requires only on- and off-axis extrusive thicknesses $\left(T_{o n}\right.$ and $\left.T_{\text {off }}\right)$ to completely describe the 
width of the two distributions:

$$
\begin{aligned}
\sigma_{e}=\frac{T_{\text {off }} \sqrt{2}}{C} & \sigma_{i} & =\frac{-T_{\text {off }}}{C} \ln \left(1-\frac{T_{o n}}{T_{\text {off }}}\right) \\
\text { where } & C & =\sqrt{2 \pi}\left(\frac{t \cdot P_{d}}{w}\right)
\end{aligned}
$$

We define the zone of dike intrusion to be $2 \sigma_{i}$, which contains $95 \%$ of all events. Similarly, the zone of volcanic emplacement is defined as twice the geometric mean of the dike standard deviation (relative to the axis) and the lava standard deviation (relative to the dikes), $2 \sqrt{\sigma_{i}^{2}+\sigma_{e}^{2}}$. Dike width $(w)$ and lava flow thickness $(t)$ were both assumed to be $1 \mathrm{~m}$ [e.g., Schmincke et al., 1983; Karson et al., 1992], and 38\% of all dikes $\left(P_{d}\right)$ were allowed to form a surface eruption [Hooft et al., 1996]. Since these parameters affect the width of the two zones equally (Eq. 5.2), they do not affect their ratio $(r)$, which then becomes a measure of focused accretion. We use, as our on- and off-axis extrusive thicknesses $\left(T_{o n}\right.$ and $\left.T_{\text {off }}\right)$, the layer $2 \mathrm{~A}$ values derived from mapping the $4 \mathrm{~km} / \mathrm{s}$ contour ( $93 \pm 11$ and $411 \pm 72 \mathrm{~m}$, respectively). Although we include a thin interval of steep gradient material, the effect on the calculation is negligible. Using these values, we calculate the zone of dike intrusion to extend $\sim 200$ $\mathrm{m}$ off-axis within a zone of active lava deposition $2-3 \mathrm{~km}$ wide. The limited off-axis extent of dike emplacement relative to the lavas $(r>5)$ reinforces the idea that the two-dimensional thermal and mechanical structure of fast spreading ridges focuses melt generation and efficiently delivers it to the seafloor.

Models of crustal accretion [e.g., Pálmason, 1980; Hooft et al., 1996] also demonstrate that the width of the transition zone, and distribution of erupted material within it is directly related to the off-axis extent of dike intrusion. A broad region of diking would encompass distally deposited lavas, and hence produce a nearly monotonic increase in dike fraction over a thick depth interval. The rapid velocity increase at the top of the pillow/dike transition in this area (Fig. 5-4), and along the EPR in general (Fig. 5-16), is indicative of a narrow zone of dike emplacement, and further 
supports a model of focused accretion at fast spreading ridges. Hooft et al. [1996] calculate that the thickness of the transition zone is generally equal to or greater than its on-axis depth. Hole 504B contains a 209-m-thick transition [Becker et al., 1989] and ophiolite studies commonly document a 100 to 200-m-thick mixed layer [Nicolas, 1989]. We therefore argue that the secondary velocity jump 250-350 $\mathrm{m}$ below the rise crest (Fig. 5-4) corresponds to the base of a variable, $\sim 200$-m-thick transition zone that overlies nearly $100 \%$ dikes.

What process is responsible for generating the velocity contrast intermittently observed at the base of the transition zone? One possible explanation is that the rheological differences between massive, columnar intrusives and more brecciated, horizontally layered volcanics may produce a mechanical boundary between fundamentally different styles of deformation. Magnetic measurements at Hole 504B reveal that the stable inclination of the extrusive section is rotated by over $20^{\circ}$ from expected, and that this mismatch is reduced within the transition zone to nearly $0^{\circ}$ in the upper dikes [Smith and Banerjee, 1986; Kinoshita et al., 1989; Pariso and Johnson, 1989]. The authors infer from these observations that the volcanic section was tectonically rotated as it moved away from the ridge axis, whereas the sheeted dikes were deformed in such a way that they remained vertically oriented. By studying the fracture evolution of the recovered drill samples, Agar [1990] found evidence for numerous mechanical discontinuities within the upper crust that separate units of varying rotational history. They also discovered a highly tectonized interval at the base of the transition zone, which they interpret to be a detachment fault. Agar and Klitgord [1995] propose that relief on lithological boundaries and the rheological contrasts across them may promote the formation of decoupling surfaces at various depths in the oceanic lithosphere, not just the pillow/dike boundary. The fault zone at Hole 504B indicates that the downward transition from cataclastic flow and rotation in the extrusives to faulting along vertically oriented dike contacts may occur abruptly, thereby generating a seismically observable impedence contrast. In areas 
where this seismic horizon is undetected, the decoupling may be accommodated across a thicker interval (i.e., Fig. 5-16a,c and the 10-13 km mark in Figs. 5-4, and Fig. 5-17). A consequence of this model is that dikes within the transition zone should also be rotated, with the degree of rotation dependent on the off-axis location they were deposited. Supporting evidence is found at the Troodos ophiolite in Cyprus, where the dikes and lava flow units within the transition zone (termed the "basal group") are significantly rotated with respect to the underlying, vertically oriented sheeted dikes [Xenophontos and Malpas, 1987]. Significant rotations are also observed in the uppermost dikes at Hess Deep, which Karson et al. [1992] propose may be the result of rapid near-axis subsidence as the uppermost crust thickened.

Another source of the deeper velocity contrast may be the differing effect that the two major lithologies have on dike propagation. The transport of lava is likely to be focused vertically along the dominant orientation of sheeted dikes, whereas magmatic cracking in the porous, horizontally layered volcanics may be less directed. This would produce a more diffuse melt channel within the extrusives, and numerous sills at the base of the transition zone, where horizontal stratigraphy is first encountered by the propagating dikes. Submersible observations show that the dikes exposed at Hess Deep are more sinuous in the lava unit than deeper in the section [Karson et al., 1992], and that massive subhorizontal layers exist in the transition zone [Francheteau et al., 1992]. Similar massive units were recovered from the transition zone of Hole 504B [Anderson et al., 1982].

Within the resolution of the data, we find no evidence that the on-axis velocity contrast at the base of the transition zone is maintained off-axis. Forward models show that, despite the increased water depth, thickened extrusive pile and more variable topography off-axis, a velocity contrast of $0.5 \mathrm{~km} / \mathrm{s}$ or greater at the base of a 200-m-thick transition zone would still be detected at the sea surface. The inability of the GA to resolve this event off-axis may be a consequence of sampling a horizon that is formed intermittently, and therefore absent in certain areas. It is also pos- 
sible that velocities within the transition zone are rapidly modified by the vigorous hydrothermal circulation thought to permeate this magmatically robust segment. If hydrothermal mineralization is responsible for the $0.6 \mathrm{~km} / \mathrm{s}$ increase in the uppermost extrusives within $6 \mathrm{~km}$ of the axis (Table 5.1), it is reasonable to suggest that the initial $0.5 \mathrm{~km} / \mathrm{s}$ contrast above the sheeted dikes is subdued by the same process. In fact, extensive alteration of the transition zone is documented at Hole 504B [Alt et al., 1986], and in exposures of the upper crust, both on land [Swift and Johnson, 1984; Johnson and Pariso, 1987] and at the seafloor. Based on drill-hole observations, Becker et al. [1989] suggest that the transition zone acts as a hydrothermal mixing front between fluids upwelling through the sheeted dikes and cooler seawater circulating within the porous extrusives. If the transition zone is also a mechanical discontinuity, Agar [1990] suggests that enhanced fluid flow along the boundary would promote the rapid mineralization of this interval.

Figure 5-21 depicts the seismic evolution of the upper crust in terms of the geological processes discussed above. Extrusives deposited on-axis, possibly within an axial summit trough, are incorporated into the pillow/dike transition zone within a narrow region of dike injection. These units rapidly subside beneath a thickening extrusive layer that is built by overflow of the axial summit trough and deposition near the axis, but outside the dike injection zone. Since seismic studies do not image the dikes reaching the seafloor, initial subsidence is thought to be too rapid to resolve (within the Fresnel zone of $\sim 300 \mathrm{~m}$ at the seafloor). Deformation is accommodated by cataclastic flow and rotation in the extrusives and transition zone, and by cataclastic faulting in the sheeted dikes. Outside the narrow corridor of dike subsidence, the on-axis seismic structure is characterized by $\sim 100$ meters of low-velocity extrusives (2.1-2.4 $\mathrm{km} / \mathrm{s}$ ) overlying a sharp transition to diabase dikes mixed with proximally deposited extrusives. This constitutes seismic layer $2 \mathrm{~A}$. A deeper velocity contrast may exist at the base of the transition zone in areas where the change in deformation styles is abrupt and possibly accompanied by sill emplacement. As the crust is transported 


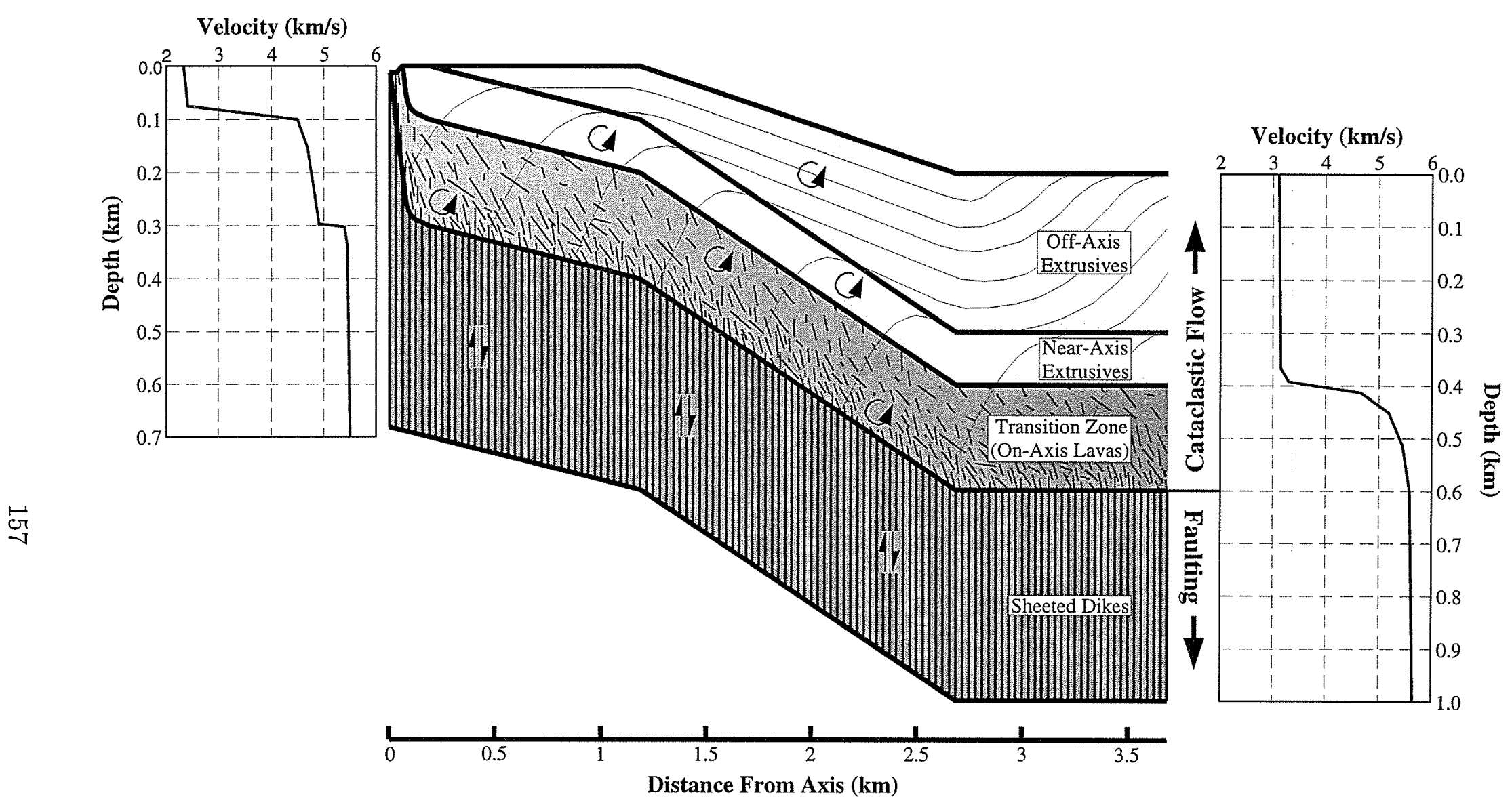

Figure 5-21: Cartoon depicting the geological processes responsible for the shallow seismic structure of fast spreading ridges. Dimensions are approximate, with a vertical exaggeration of 2.5. Extrusives are divided into three depositional domains: onaxis (transition zone), near-axis, and off-axis. Thin lines represent isochrons, and thick lines track flow paths between volcanic domains. Gray shading roughly maps the depth- and age-dependent increase in seismic velocity. Two velocity functions depict the seismic structure observed on- and off-axis. The zone of rapid subsidence at the rise crest is thought to be too narrow to image seismically. See text for discussion. 
away, off-axis lava deposition further deforms layer $2 \mathrm{~A}$ and thickens it by a factor of four to five within 1-4 km of the rise axis. Simultaneously, hydrothermal processes modify the initial porosity structure of the shallow layers, raising their seismic velocities by over $0.5 \mathrm{~km} / \mathrm{s}$ within $6 \mathrm{~km}$ of the rise axis. Pervasive mineralization within the transition zone may also subdue any velocity contrast initially formed at its base, rendering it seismically transparent. Continued hydrothermal circulation raises basement velocities to near $5 \mathrm{~km} / \mathrm{s}$ within $10 \mathrm{Myr}$ and produces seismically identifiable metamorphic fronts within the extrusive section, as observed at Hole 504B.

\subsection{Summary and Conclusions}

Using a genetic algorithm-based inversion technique, we have examined the seismic velocity structure along five multichannel lines from the EPR at $17^{\circ} 20^{\prime} \mathrm{S}$. The success of our analysis was facilitated by the continuous coverage that the multichannel data provides and the GA's efficient use of seismic information to construct accurate velocity models. By mapping the shallow structure of young, fast-spreading crust with unprecedented detail, we have demonstrated the ability of single-ship MCS studies to incorporate reflectivity and velocity information into a more complete structural model of the subsurface. The major scientific results of this study are as follows:

1. A 70 to 100 -m-thick surficial low-velocity layer $(2.1-2.4 \mathrm{~km} / \mathrm{s})$ exists at the rise crest of the $17^{\circ} 20^{\prime} \mathrm{S}$ study area. This feature is common, if not ubiquitous, along the fast spreading EPR, with little variation in velocity or thickness. Because of its uniformly low velocities, we equate this layer with pure extrusives, below which a sharp increase in dike fraction generates a seismic velocity boundary that can be continuously mapped for tens of kilometers. We label this horizon as the base of seismic layer $2 \mathrm{~A}$, and note its shallow location with respect to previous estimates of layer 2A thickness $(150-250 \mathrm{~m})$.

2. Also on-axis, a second, smaller velocity contrast is intermittently observed at a 
variable basement depth of 250-320 m. The depth of this horizon closely corresponds to previous layer $2 \mathrm{~A}$ thickness estimates. Its occurrence outside our study area $\left(9^{\circ} \mathrm{N}\right)$, and clear separation from the seafloor event suggest that this event may be the source of thick layer $2 \mathrm{~A}$ estimates elsewhere along the EPR. Based on direct seafloor observations, borehole evidence, and theoretical constraints, we place this horizon at the base of the pillow/dike transition, where the change in rheology, depositional history and possibly deformation style may generate an abrupt impedence contrast.

3 . The continued off-axis accumulation of lavas thickens layer $2 \mathrm{~A}$ by an additional $300-400 \mathrm{~m}$ within $1-4 \mathrm{~km}$ of the rise axis. This extrusive thickening profile predicts a pattern of focused melt delivery in which a narrow zone of on-axis dike intrusion ( $\sim 200 \mathrm{~m}$ half-width) feeds lava flows that typically extend up to $1.5 \mathrm{~km}$ from the rise axis.

4. An increase in extrusive velocities of $0.6 \mathrm{~km} / \mathrm{s}$ within $6 \mathrm{~km}$ of the rise crest is attributed to the cementation of small-scale porosity by hydrothermal alteration products. This is faster than for the EPR as a whole and may indicate that the level of hydrothermal activity is elevated at this magmatically robust ridge segment. We show that extrusive velocities near $5 \mathrm{~km} / \mathrm{s}$ are reached within $10 \mathrm{Myr}$ of formation, and that this increase can be described by a square root of age relation.

5. Within the resolution of our data, no double-step velocity structure is observed off-axis. Although the initial formation of such a pattern is not certain, we propose that hydrothermal mineralization is enhanced in the transition zone, and would therefore decay any initial velocity contrast at the base of the transition zone. 


\section{Chapter 6}

\section{Upper crustal seismic structure of}

the slow spreading Mid-Atlantic Ridge, $35^{\circ} \mathrm{N}$ : Constraints on volcanic emplacement processes

\subsection{Introduction}

Geophysical evidence accumulated over the last three decades convincingly suggests that the style of melt generation and crustal accretion at slow spreading ridges is fundamentally different than at their faster spreading counterparts [Macdonald, 1986, 1989; Solomon and Toomey, 1992; Forsyth, 1992; Sparks and Parmentier, 1994]. Bathymetry [Sempéré et al., 1993; Thibaud et al., 1997], gravity [Kuo and Forsyth, 1988; Lin et al., 1990; Rommevaux et al., 1994; Detrick et al., 1995], and seismic data [Sinha and Louden, 1983; Tolstoy et al., 1993] collected at slow spreading ridges reveal a pattern of pronounced crustal thickening $(1-4 \mathrm{~km})$ beneath spreading segment centers relative to their distal ends. No such pattern has been documented at fast spreading ridges. Coupled with numerical models [Parmentier and Phipps Morgan, 1990], these observations suggest a transition from two-dimensional, plate-driven 
mantle flow and/or crustal accretion at fast spreading rates to three-dimensional, buoyant mantle upwelling at slower spreading rates [Whitehead et al., 1984; Lin and Phipps Morgan, 1992]. The rift valley morphology common to the slowly spreading Mid-Atlantic Ridge (MAR) indicates that the lithosphere is at a cooler thermal state, especially near segment offsets, and is therefore mechanically stronger than at faster spreading rates [Neumann and Forsyth, 1993; Phipps Morgan and Chen, 1993]. This concept is supported by the lack of seismic evidence for a long-lived intracrustal magma chamber beneath the rift valley of the MAR [e.g., Fowler, 1976, 1978; Toomey et al., 1988; Detrick et al., 1990], and by the regular occurrence of seafloor morphologies dominated by tectonic extension rather than magmatic accretion [Karson and Dick, 1983; Macdonald, 1986; Tucholke and Lin, 1994].

Relatively little is known about how these differences in crustal structure and mantle upwelling manifest themselves, if at all, in the formation and internal structure of the volcanically emplaced upper crust. Extensive observations of the rift valley floor [e.g., Ballard and Van Andel, 1977; Smith and Cann, 1992; Searle et al., 1998] have yielded valuable information on the emplacement and modification of the extrusive section, but deeper structure can only be inferred from the surface expression of this layer. Multichannel seismics, arguably the best method for directly measuring the internal structure and thickness of the extrusive crust, is severely hampered at slow spreading ridges by the extreme topography of the rift valley environment [e.g., Detrick et al., 1990; Calvert, 1997]. Consequently, the bulk of our understanding about the basaltic crust has come from fast spreading ridges such as the East Pacific Rise (EPR).

Numerous refraction studies along the East Pacific Rise axis reveal a thin $(<125 \mathrm{~m}$ thick) low-velocity layer immediately beneath the seafloor that is surprisingly uniform in thickness along the rise axis [Harding et al., 1989; Vera et al., 1990; Detrick et al., 1993; Christeson et al., 1994a; Kappus et al., 1995; Tolstoy et al., 1997; Ch. 5]. This section, thought to be pure extrusives, is underlain by a $100-200 \mathrm{~m}$ thick transition to 
velocities typical of the diabase dikes which feed it. In some locations, this transition is a single gradient (e.g., $13^{\circ} \mathrm{N}$ [Harding et al., 1989; Kappus et al., 1995] and $14^{\circ} \mathrm{S}$ [Tolstoy et al., 1997]), and at others it is double-step in character (e.g., $9^{\circ} \mathrm{N}$ [Vera

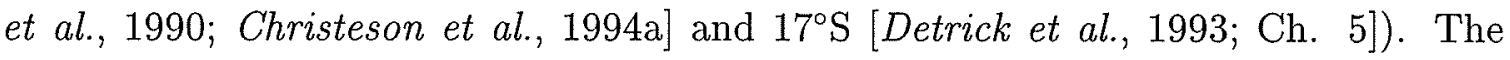
low-velocity and transition layers together form what is termed seismic layer $2 \mathrm{~A}$, below which lie the relatively uniform dike velocities of layer 2B. Multichannel seismic (MCS) profiles have shown that layer $2 \mathrm{~A}$ typically doubles in thickness within $\sim 3 \mathrm{~km}$ of the rise axis [Harding et al., 1993; Kent et al., 1994; Carbotte et al., 1997; Hooft et al., 1997a] - a geometry that has recently been modeled by Hooft et al. [1996] through the repeated deposition of lavas. The detailed velocity analysis of Chapter 5 suggests that this thickening is accomplished by a four- to five-fold buildup of pure extrusives away from the axial peak, and a thinning of the seismic transition zone due to hydrothermal infilling of pores/cracks.

A more limited number of seismic studies have examined the upper crust built at the intermediate spreading Juan de Fuca Ridge (JDFR) [Poujol and Jacobson, 1988; Rohr et al., 1988; White and Clowes, 1990; Cudrack and Clowes, 1993; McDonald et al., 1994]. They reveal a thicker, more variable layer $2 \mathrm{~A}$ than at the EPR. Refraction studies of the Endeavor segment at $48^{\circ} \mathrm{N}$ [White and Clowes, 1990; Cudrack and Clowes, 1993] find layer $2 \mathrm{~A}$ to be $200-650 \mathrm{~m}$ thick, with velocities of $2.5-2.8 \mathrm{~km} / \mathrm{s}$. An abrupt increase in velocity beneath this layer is equated with an intermittent reflector imaged $0.3-0.5 \mathrm{~s}$ beneath the seafloor along a coincident multichannel line [Rohr et al., 1988]. Refraction data from the Cleft segment at $45^{\circ} \mathrm{N}$ also document a highly variable layer $2 \mathrm{~A}(200-550 \mathrm{~m}$ thick, $\sim 2.7 \mathrm{~km} / \mathrm{s})$ that is $\sim 350 \mathrm{~m}$ thick beneath the rise axis, but exhibits no systematic cross- or along-axis trends $M c D o n a l d ~ e t ~ a l$. [1994]. Thickness estimates of the magnetic source layer in this region correlate well with the seismic layer 2A results [Tivey, 1994].

The few studies that have resolved upper crustal structure at the Mid-Atlantic Ridge have discovered a thick, variable layer $2 \mathrm{~A}$ structure similar to that at the Juan 
de Fuca Ridge. The first direct evidence of a surficial low-velocity layer at the MAR was recorded by Purdy and Detrick [1986] during a conventional refraction study at $23^{\circ} \mathrm{N}$. Their preferred model includes a 450 -m-thick low-velocity interval (2.55 $\mathrm{km} / \mathrm{s}$ ) overlying a discontinuous jump to higher velocities. Purdy [1987], using onbottom sources and receivers, confirmed the existence of this layer, and documented an increase in its seismic velocity from $2.1 \mathrm{~km} / \mathrm{s}$ within the median valley to $4.1 \mathrm{~km} / \mathrm{s}$ in $7 \mathrm{Myr}$-old crust. In contrast to these refraction experiments, previous multichannel studies have been less successful at imaging energy that turns at the base of layer $2 \mathrm{~A}$ [Detrick et al., 1990; Calvert, 1997; Smallwood and White, 1998]. However, Smallwood and White [1998] constructed a layer 2A thickness profile from MCS data along the Reykjanes Ridge by hand fitting two-layer constant velocity models to refractions recorded on selected common depth point (CDP) gathers. Using a constant seafloor velocity of $2.45 \mathrm{~km} / \mathrm{s}$, they measured the average thickness of this highly variable layer at $400 \pm 100 \mathrm{~m}$. No age-dependent velocity trends or systematic thickness variations were resolved. A similar lack of consistent velocity trends in tomographic data from the rift valley at $35^{\circ} \mathrm{N}$ led Barclay et al. [1998] to infer that the axis of extrusion is highly variable on timescales of $25 \mathrm{kyr}$ or less. Anomalously low velocities in the shallow crust of this area further suggest that the transition to seismic layer $2 \mathrm{~B}$ occurs at a greater depth than at the EPR [Barclay et al., 1998]. As at the JDFR [McDonald et al., 1994], the significant anisotropy recorded in the upper crust of the MAR is most likely due to ridge-parallel fractures [Barclay et al., 1998]. This observation reflects the increased role of tectonic extension at slower spreading rates.

In this study, we present the first detailed investigation of seismic layer $2 \mathrm{~A}$ structure at the MAR using multichannel seismics. After introducing the study area and outlining our analysis techniques, we examine the data and present upper crustal velocity models for selected locations in the survey area. These models are then embedded within conventionally processed seismic profiles to better interpret the seismic structures they resolve. Finally, we discuss the implications of our results on the em- 
placement of extrusive crust at slow spreading centers and compare the upper crustal seismic structure of this area to that of the fast spreading East Pacific Rise.

\subsubsection{The Study Area}

Situated south of the Azores platform, the Oceanographer (at $35.2^{\circ} \mathrm{N}$ ) and Hayes $\left(\right.$ at $33.6^{\circ} \mathrm{N}$ ) transforms bound three distinct spreading segments separated by rightstepping non-transform offsets (NTO). The full spreading rate at this part of the Mid-Atlantic Ridge is constrained by magnetic anomaly picks [LeDouaran et al., 1982] and plate motion models [e.g., DeMets et al., 1990] to be $21-22 \mathrm{~mm} / \mathrm{yr}$. Multibeam bathymetry data obtained during the 1991 FARA-SIGMA cruise [Needham et al., 1991] reveals the northernmost segment, termed OH-1, to be hourglass-shaped in map view - a morphology that has typically been associated with enhanced and focused mantle upwelling at these slow spreading rates [Sempéré et al., 1993; Thibaud et al., 1997] (Fig. 6-1). The inner valley floor narrows at the segment center, and shoals by over $1 \mathrm{~km}$ to just over $2 \mathrm{~km}$ in depth, resulting in a cross-axis relief of only $\sim 500 \mathrm{~m}$. In addition, this segment contains a robust constructional volcanic ridge that stands up to $300 \mathrm{~m}$ above the inner valley floor. This morphology is in contrast to the deep, tectonically dominated rift valleys of the southern two segments, whose inner valley floors only slightly shoal toward the center and are largely devoid of any robust constructional ridges.

Sea surface gravity measurements from the FARA-SIGMA cruise show that segment $\mathrm{OH}-1$ is associated with one of the largest mantle Bouger anomalies, or "bull's eyes", of any segment along the northern MAR [Detrick et al., 1995]. The source of this gravity low is inferred by Detrick et al. [1995] to be a substantial thickening $(\sim 5 \mathrm{~km})$ of crust toward the segment center. This interpretation is supported by the seismic refraction results of Sinha and Louden [1983], which indicate that crust underlying the eastern rift mountains thins from $9-10 \mathrm{~km}$ near the middle of the segment to $<6 \mathrm{~km}$ at the ends. Such gravity lows and crustal thickness variations 


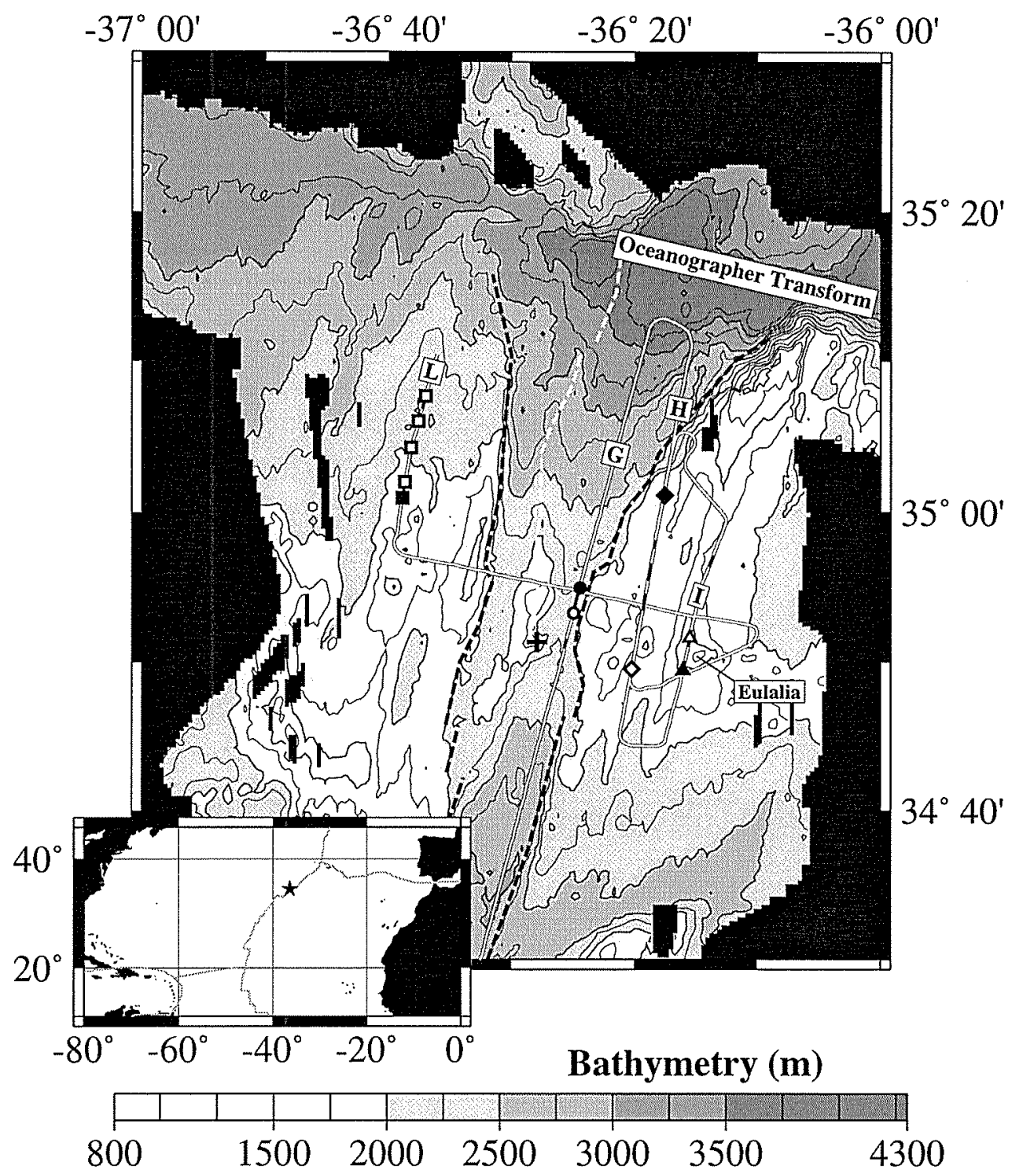

Figure 6-1: Bathymetric map of OH-1 (star in inset), the northernmost spreading segment between the Oceanographer and Hayes transforms (merged data from the FARA-SIGMA [Needham et al., 1991] and MARBE studies [A. Hosford, unpublished data]. MCS track lines (white) were shot along the morphotectonic grain and are black in areas where the bathymetry is subdued and shallow enough to allow stacking of adjacent gathers. Refractions through layer $2 \mathrm{~A}$ are observed in the rift mountains and the shallow, central portion of the rift valley. Profiles are labeled alphabetically in order of acquisition, and were shot along 0, 0.7, 1.6, and 1.9 Myr-old crust, respectively. Symbols mark where the data were of high enough quality to allow a successful velocity analysis: two along line $G(0)$, two along $H(\diamond)$, two along $I(\triangle)$ and five along $L(\square)$. The detailed results at one site per line (filled symbols) are shown later. Black dashed lines mark the first major bounding faults on either side of the rift valley floor and a white dashed line follows the main axial volcanic ridge northward from the subsurface low-velocity body detected by Barclay et al. [1998] (cross). 
are further evidence that segment $\mathrm{OH}-1$ is experiencing voluminous, sustained and focused magma upwelling and melt production [Lin et al., 1990].

The center of OH-1 was one of two study sites explored by the submersible Nautile during the 1995 OCEANAUT cruise [Bideau et al., 1996]. These dives find no evidence of tectonic activity within the inner valley floor, which suggests that the rift valley is dominated by magmatic processes at the segment center. A single uplifted scarp, which may be a juvenile bounding fault forming within the rift valley, splits the southern portion of the constructional ridge and has confined recent volcanic emplacement within the eastern trough. This is confirmed by the presence of fresh lavas east of the median ridge and more sedimented flows in the western trough.

The shallowest of the median ridge edifices is bisected by this juvenile bounding fault (Figure 6-1). It is beneath this seamount that anomalously low seismic velocities were recorded during the 1991 FARA microearthquake experiment [Purdy et al., 1993; Barclay et al., 1998], suggesting the presence of partial melt. However, Barclay et al. [1998] found no evidence in the seismic travel time delays for off-axis deepening of the high-velocity dikes, as observed at fast spreading centers. They interpret this as indicating that the axis of volcanic accretion at $\mathrm{OH}-1$ does not remain stable on timescales greater than $25 \mathrm{kyr}$.

A line of seamounts intersects the median ridge at its shallowest point and extends parallel to the spreading direction out to the limit of bathymetric coverage on both sides of the axis (Fig. 6-1). Submersible observations by Bideau et al. [1996] reveal that these off-axis seamounts are heavily sedimented and of the same magnetic polarity as the surrounding crust, indicating that they formed near-axis and were subsequently rafted out of the rift valley intact. Seven kilometers west of the median ridge, a large-throw normal fault uplifts the crust to depths of less than $1.5 \mathrm{~km}$ in the rift mountains. To the east, a more gradual transition to similar depths is punctuated by an 11-km-wide intermediate terrace containing several small constructional edifices. A large seamount, dubbed Eulália volcano by Bideau et al. [1996], sits at 
the western edge of the upper terrace and rises to within $1 \mathrm{~km}$ of the sea surface. The rift mountains bordering both the ridge axis and active transform (inside corner) are bathymetrically shallower and exhibit a larger mantle Bouger anomaly than their cross-axis counterparts (outside corner) [Detrick et al., 1995]. This segmentscale asymmetry is not uncommon along the MAR, and is thought to be a result of the removal of upper crust by a detachment fault and subsequent isostatic uplift of the inside corner, aided by the decoupling effects of both the ridge axis and active transform [Severinghaus and Macdonald, 1988; Tucholke and Lin, 1994; Escartin and Lin, 1995].

\subsection{Data Collection and Analysis}

In the fall of 1996, multichannel seismic data were acquired between the Oceanographer and Hayes transforms during four days of the Mid-Atlantic Ridge Bullseye Experiment (MARBE) [Detrick et al., 1997]. The goals of the MCS study were to evaluate the effectiveness of new processing methods that suppress the scattered energy generated by the rugged topography of the MAR [G. Kent, in preparation] and to image the velocity structure of the shallowmost crust (this study).

MCS lines were shot along the rift valleys of all three major spreading segments, and parallel to the ridge in the rift mountains of OH-1 (Fig. 6-1). Since seafloor topography is most subdued along the morphotectonic grain, lines were shot in this orientation to minimize the amount of scattered energy recorded (Fig. 6-2). Data were acquired by the RV Maurice Ewing's 160-channel, $4 \mathrm{~km}$ long digital streamer. To achieve a nominal shot spacing of $33 \mathrm{~m}$, a towing speed of 3.75 knots was attempted, with the 10-gun, 3005 cubic inch airgun array firing every $16 \mathrm{~s}$. The airguns were tethered to floating buoys at a depth of $10 \mathrm{~m}$ so as to maintain a repeatable source waveform. This configuration yielded a densely spaced data set of $\sim 60$-fold coverage.

Because of the shallow water depths associated with proximity to the Azores 

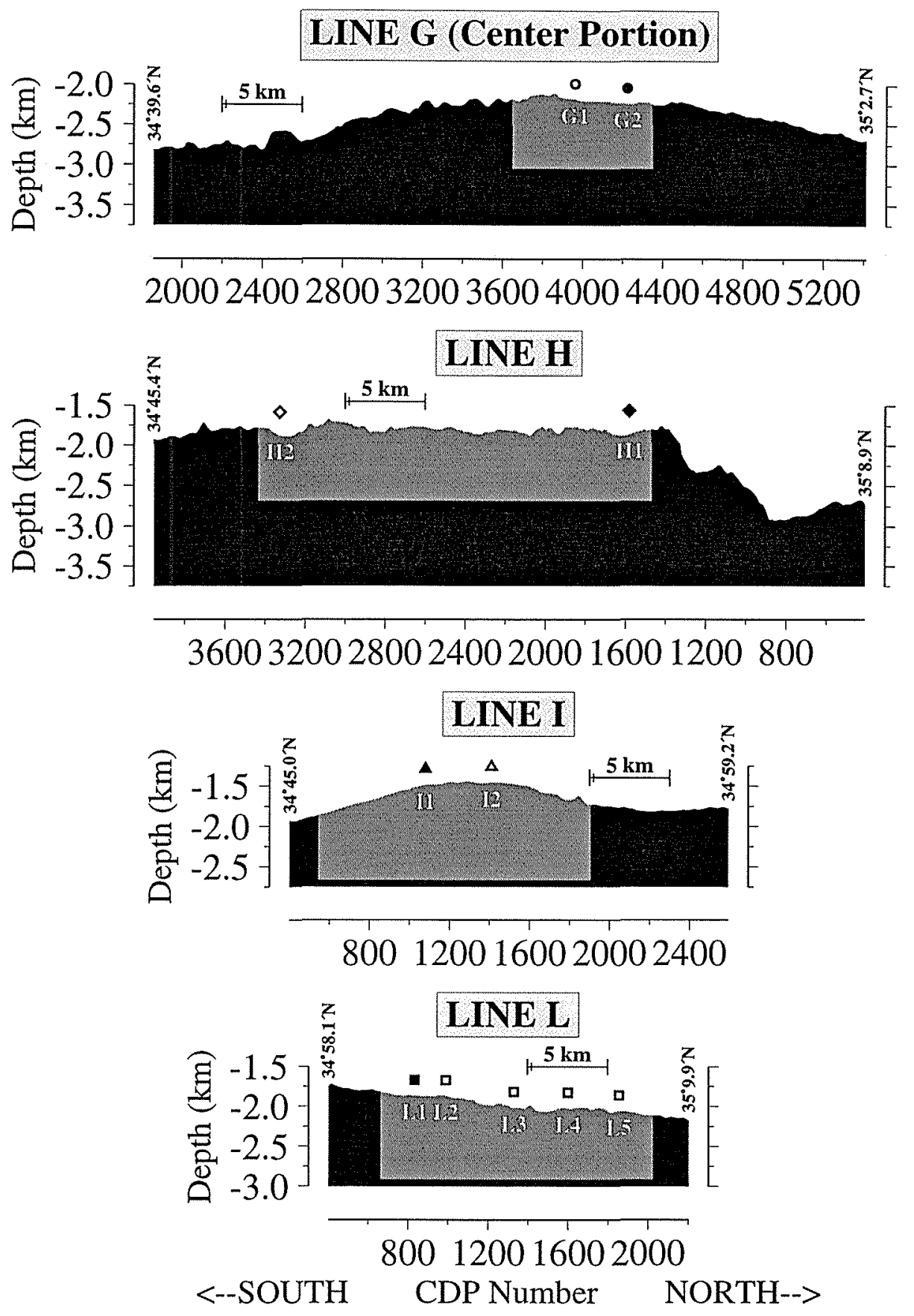

Figure 6-2: Bathymetric profiles along the four north-south MCS lines. All are plotted at the same scale, with a 5:1 vertical exaggeration. Symbols are as in Figure 6-1. Note the flatlying character of the seafloor, especially in areas where a detailed velocity analysis is performed. Twenty CDPs were summed at each location, the width of which is marked by the two leftmost tickmarks on the scale bars. Grey regions enclose both the depth to which our velocity analysis is sensitive $(\sim 1 \mathrm{~km})$ and the lateral extent of the seismic sections in Figure 6-15. 
hotspot, refractions from the uppermost crust were clearly recorded along all rift mountain profiles and within the shallowest parts of the $\mathrm{OH}-1$ median valley. The great rift valley depths of the southern segments $(>3 \mathrm{~km})$ prevented shallow refractions from being recorded within the limited aperture of the streamer. Based on the Hydrosweep centerbeam depth and the raw seismic images (stacked at only the water velocity of $1500 \mathrm{~m} / \mathrm{s}$ ), flatlying sections of seafloor were identified. Of these, eleven exhibit coherent, identifiable upper crustal refractions and are topographically subdued enough to allow the stacking of adjacent common midpoint (CMP) gathers without adversely affecting the data (Figs. 6-1 \& 6-2). Two are situated at the shallow, central part of the inner valley floor (line G), two sit at the northern and southern end, respectively, of the intermediate terrace east of the rift valley (line $\mathrm{H}$ ), two straddle the flanks of Eulália volcano on the upper terrace (line I), and five are aligned along an axis-parallel topographic high in the western rift mountains (line L). These locations were chosen for a detailed velocity analysis.

To enhance the signal-to-noise ratio, supergathers were constructed at these locations by summing $20 \mathrm{CMP}$ gathers ( $250 \mathrm{~m}$ lateral extent). In each supergather, energy turning within the high-gradient transition to layer $2 \mathrm{~B}$ velocities - the primary focus of this analysis-laterally samples only $0.4-1.0 \mathrm{~km}$ of this layer, and encompasses only $1.7-2.5 \mathrm{~km}$ of seafloor, depending on the water depth. Traces were band-pass filtered between 5 and $40 \mathrm{~Hz}$ to remove low-frequency strumming of the streamer and

Figure 6-3: (next page) Data supergathers for on-axis line G (a,b), eastern terrace line $\mathrm{H}$ $(b, c)$ and eastern rift mountain line $I(e, f)$. All are plotted at the same amplitude scale, with arrivals preceding the seafloor reflection enhanced by a factor of five. Upper crustal refractions are outlined by black and white curves, and labeled as follows: "2Ap", prograde energy from the surficial low-velocity layer, " $2 \mathrm{~A}$ ", retrograde energy from the underlying velocity gradient (split into " $\mathrm{a}$ " and " $\mathrm{b}$ " branches where necessary), and "2B", prograde energy from the high-velocity layer beneath this transition. " $2 \mathrm{Am}$ " is a multiple of " $2 \mathrm{~A}$ ". Synthetic seismograms were compared to the data only within these windows. Note how the shallow bathymetry in the rift mountains brings refractions to close ranges and travel times relative to the seafloor reflection. 
a) Supergather G1: CDPs 3955-3975

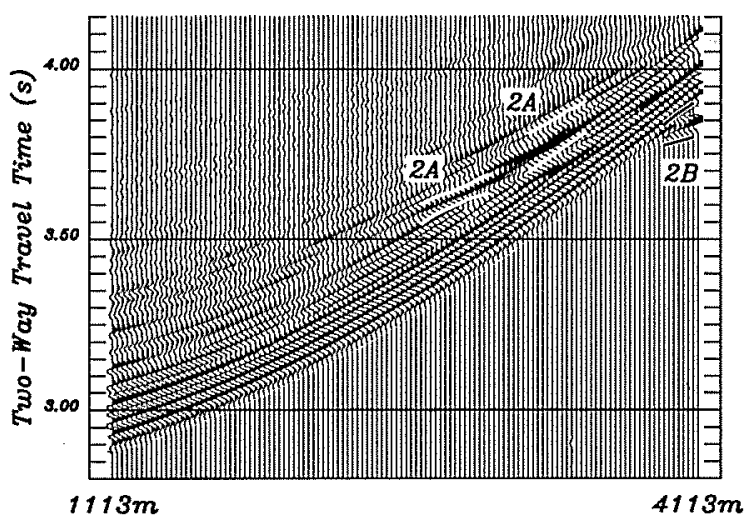

c) Supergather H1: CDPs 1570-1590

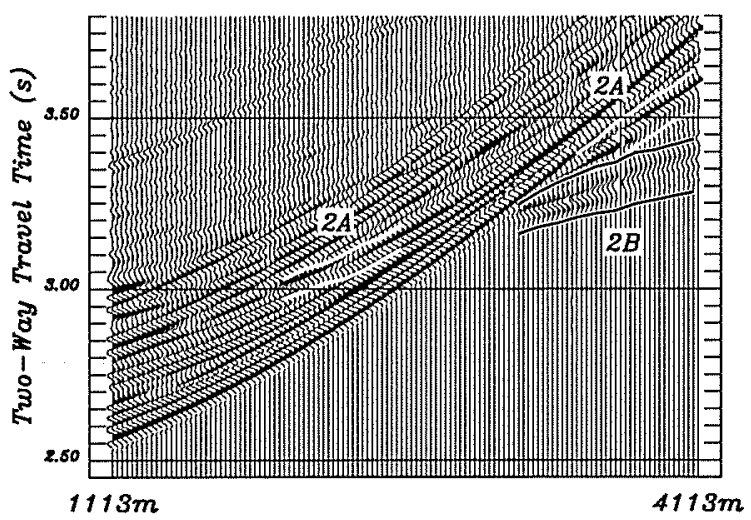

e) Supergather 11: CDPs 1070-1090

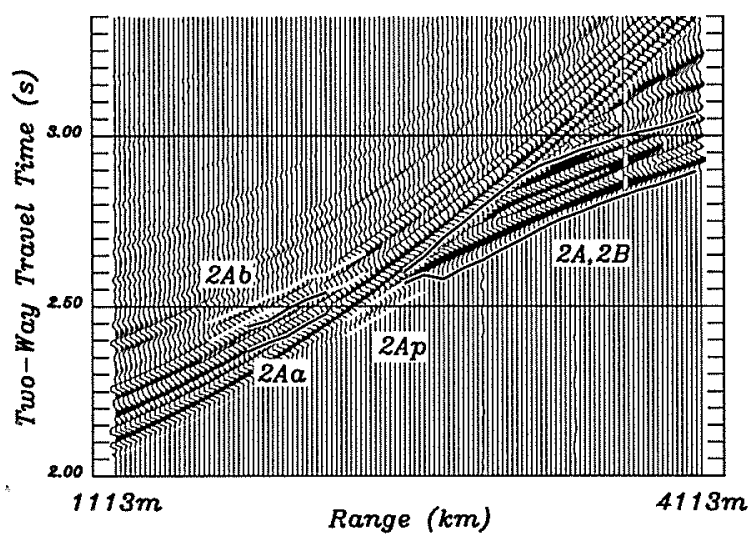

b) Supergather C2: CDPS 4215-4235

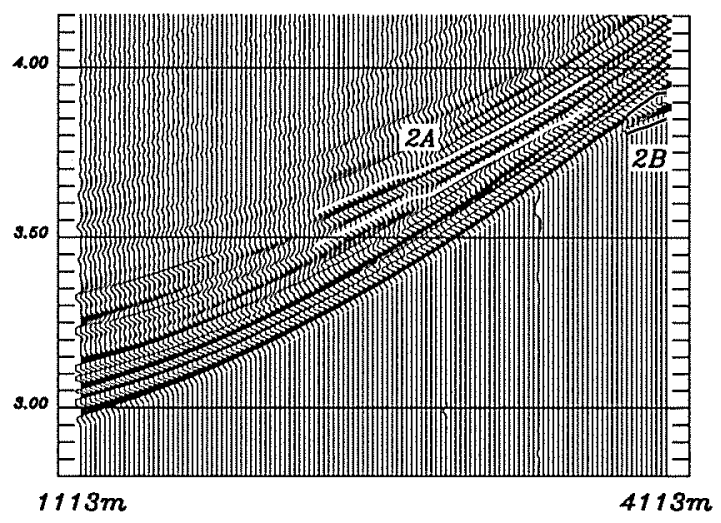

d) Supergather H2: CDPs 3315-3335

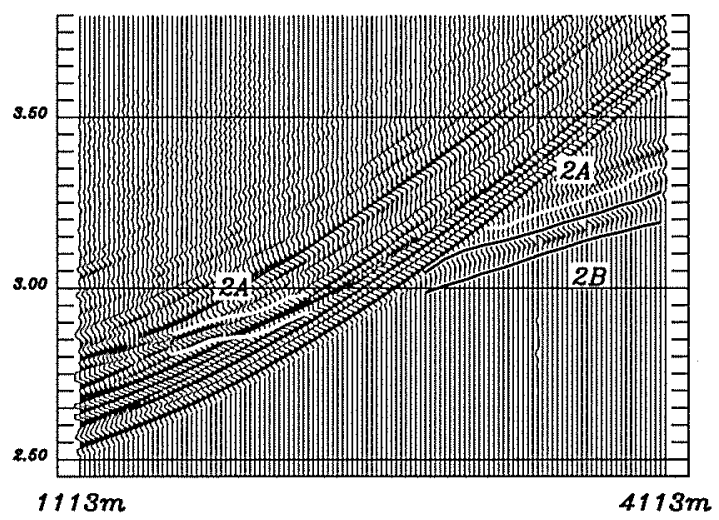

f) Supergather I2: CDPS 1400-1420

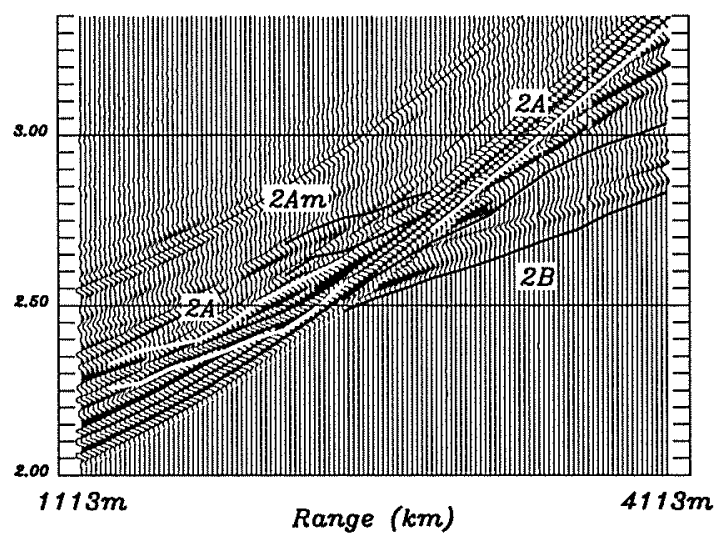



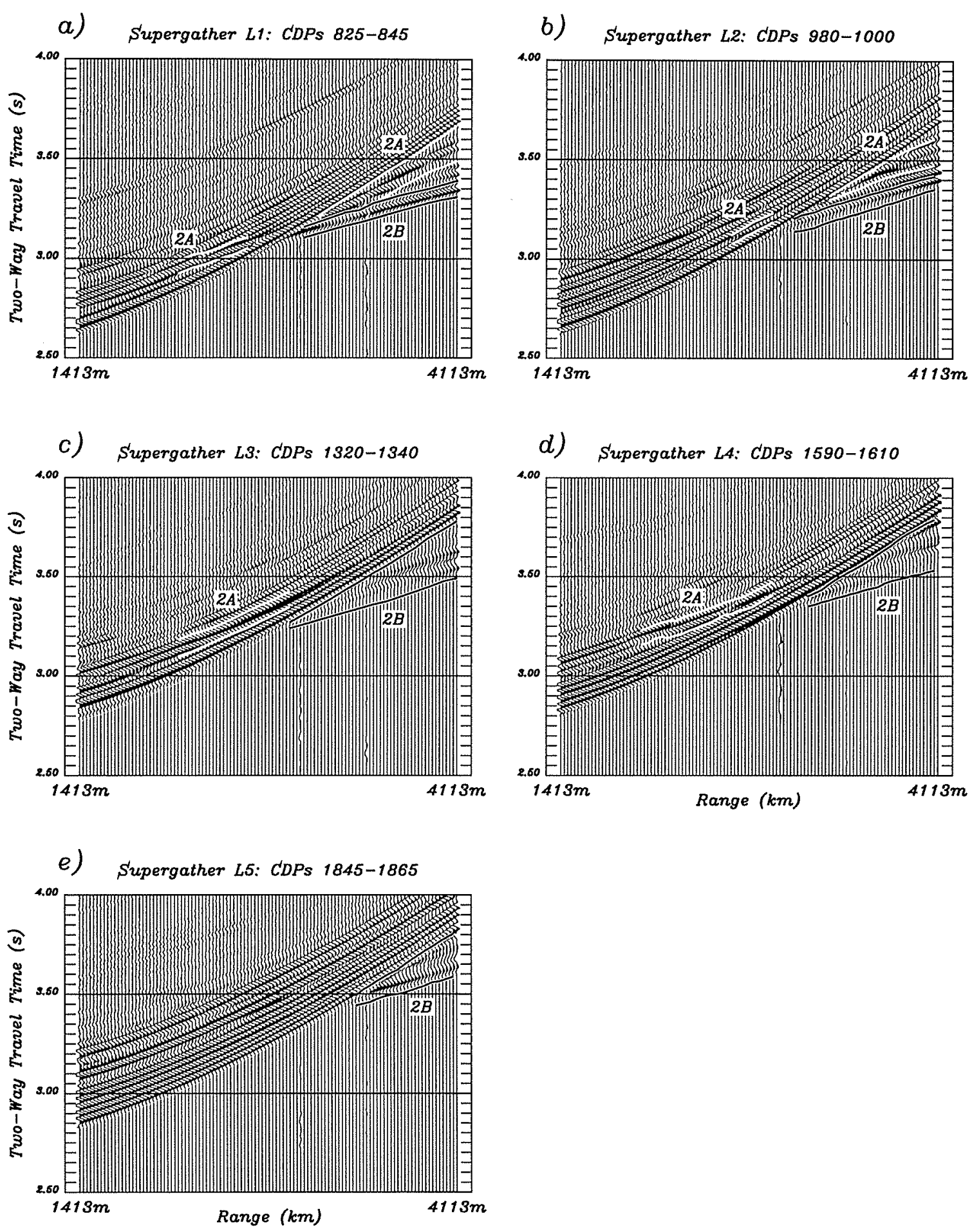

Figure 6-4: Same as Figure 6-3, except for western rift mountain line L. The gradual deepening of the seafloor to the north diminishes the amount of refraction data available. 
spurious high-frequency noise. Finally, upper crustal refractions were identified (Figs. $6-3 \& 6-4)$, and modeled by an automated waveform inversion in the form of a genetic algorithm (GA).

The GA is a pre-stack velocity inversion technique that has successfully been applied to two-ship multichannel data [Harding and Kappus, 1996; Tolstoy et al., 1997], and recently to single-ship data [Ch. 5] from the East Pacific Rise. As in the previous chapter, we use the GA to iteratively construct populations of velocity models and compare their corresponding synthetic seismograms to the data. The WKBJ method of Chapman [1978] was used to calculate all synthetics, which were then convolved with a source waveform and compared to the data seismograms only within the windows encompassing identified upper crustal refractions (Figs. 6-3 \& $6-4)$.

Based on the concept of biological natural selection, the GA robustly searches model space for a global optimum by using components of better fitting models as building blocks for new models [Goldberg, 1989; Davis, 1991; Sen and Stoffa, 1995]. For a more detailed discussion on the application of genetic algorithms to seismic waveform inversion, see Sambridge and Drijkoningen [1992], Sen and Stoffa [1992], Harding and Kappus [1996] and Appendix A. In essence, the success of the GA is based on its coding scheme, which uniquely describes each model. In this study, each velocity model is coded by a series of two-bit binary strings (0-3), which represents the number of depth scalings, $\delta z$, each node (i.e., layer) lies beneath the preceding one. The larger the depth scaling (i.e., lower resolution), the fewer the depth nodes required to describe a specific velocity model, and hence, the more efficient the GA becomes. We test this tradeoff between resolution and efficiency by performing two separate inversions on each supergather, one with a substantially higher depth scaling $(25 \mathrm{~m})$ than the other $(10 \mathrm{~m})$. In nearly half the cases ( 5 of 11$)$, the coarser resolution allowed the GA to more efficiently find the best fitting models (Table 6.1).

The inversion procedure used in this study is similar to that of Chapter 5. Each 
Table 6.1: Variable Parameter Settings for the Genetic Algorithm ${ }^{a}$

\begin{tabular}{|c|c|c|c|c|c|c|c|}
\hline $\begin{array}{l}\text { Location } \\
\text { ID }\end{array}$ & $\begin{array}{c}\text { Depth } \\
\text { Scaling, } \delta z\end{array}$ & $\begin{array}{c}\text { Number of } \\
\text { Depth Nodes }\end{array}$ & $\begin{array}{l}\text { Max. Depth } \\
\text { Modeled }^{c}\end{array}$ & $\begin{array}{c}\text { Number of } \\
\text { Generations }^{b}\end{array}$ & $\begin{array}{c}\text { Models } \\
\text { per Gen. }\end{array}$ & $\begin{array}{c}\text { Data } \\
\text { Multiple }^{d}\end{array}$ & $\begin{array}{l}\text { Synthetic } \\
\text { Multiple }\end{array}$ \\
\hline G1 & $10 \mathrm{~m}$ & 53 & $0.8 \mathrm{~km}$ & $100(500)$ & $40(14)$ & & \\
\hline $\mathrm{G}_{2}$ & $25 \mathrm{~m}$ & 26 & $1.0 \mathrm{~km}$ & $70(350)$ & $60(25)$ & & \\
\hline $\mathrm{H} 1$ & $10 \mathrm{~m}$ & 53 & $0.8 \mathrm{~km}$ & $100(500)$ & $40(14)$ & & $\checkmark$ \\
\hline $\mathrm{H} 2$ & $10 \mathrm{~m}$ & 53 & $0.8 \mathrm{~km}$ & $100(500)$ & $40(14)$ & & \\
\hline $\mathrm{I} 1$ & $25 \mathrm{~m}$ & 26 & $1.0 \mathrm{~km}$ & $70(350)$ & $60(25)$ & & $\checkmark$ \\
\hline $\mathrm{I} 2$ & $10 \mathrm{~m}$ & 53 & $0.8 \mathrm{~km}$ & $100(500)$ & $40(14)$ & $\checkmark$ & $\checkmark$ \\
\hline L1 & $25 \mathrm{~m}$ & 26 & $1.0 \mathrm{~km}$ & $70(350)$ & $60(25)$ & & $\checkmark$ \\
\hline $\mathrm{L} 2$ & $25 \mathrm{~m}$ & 26 & $1.0 \mathrm{~km}$ & $70(350)$ & $60(25)$ & & $\checkmark$ \\
\hline L3 & $10 \mathrm{~m}$ & 53 & $0.8 \mathrm{~km}$ & $100(500)$ & $40(14)$ & & $\checkmark$ \\
\hline $\mathrm{L} 4$ & $25 \mathrm{~m}$ & 26 & $1.0 \mathrm{~km}$ & $70(350)$ & $60(25)$ & & \\
\hline L5 & $10 \mathrm{~m}$ & 53 & $0.8 \mathrm{~km}$ & $100(500)$ & $40(14)$ & & \\
\hline
\end{tabular}

${ }^{a}$ All other GA parameters as in Chapter 5

${ }^{b}$ Values in parenthesis are for the initial seafloor velocity search run

${ }^{c}$ Equals the number of depth nodes multiplied by $1.5(\delta z)$

${ }^{d}$ Windowed behind seafloor reflection 
inversion consisted of three GA runs. The first was tailored to find the seafloor velocity by simultaneously modeling five distinct subpopulations, each with a different seafloor node (Table 6.1). The solution of each run was used as the input starting model to the next, but only the statistics of the best run were used to calculate the final solution. Only two modifications were introduced into the inversion procedure of Chapter 5 . The first is that multiples reflecting once from the underside of the seafloor were modeled where appropriate (Table 6.1). These events record energy that has traveled twice through the uppermost crust, and can therefore help constrain its structure. Even in areas that do not exhibit a high-amplitude multiple, doubly refracted energy may still significantly modify primary phases at large ranges, and must therefore be included in the synthetic calculations. The second modification is that the source waveform was attenuated prior to its convolution with the WKBJ seismograms to simulate the effects of travel through layer $2 \mathrm{~A}$ (the WKBJ calculation does not model attenuation). Intrinsic attenuation is a frequency dependent relation given by,

$$
S^{\prime}(\omega)=S(\omega) \cdot \exp \left(\frac{-\omega d}{Q V}\right)=S(\omega) \cdot \exp \left(\frac{-\omega T}{2 Q}\right)
$$

where $S$ and $S^{\prime}$ are the original and attenuated waveforms, respectively, $Q$ is the attenuation coefficient, $\omega$ is frequency, $d$ is layer thickness, $V$ is layer velocity, and $T$ is the two-way travel time through that layer. Recent estimates of $Q$ in layer $2 \mathrm{~A}$ range from 11 to 22 [Christeson et al., 1994b; Wilcock et al., 1992]. These values are low enough to significantly modify the waveforms traveling through an extrusive layer of geologically reasonable thickness. Since all of the windowed events (except for "2ap" in supergather I1) enclose energy that has turned at or below the base of layer 2A, we use the attenuated waveform shown in Figure 6-5.

In addition to analyzing the velocity structure at specific locations, a seismic profile that includes these study sites was generated along each line (grey regions in Fig. 6-2). A coarse stacking velocity model was constructed every $250 \mathrm{~m}$ (i.e., 20 CDPs) by testing moveout velocities at $0.25 \mathrm{~km} / \mathrm{s}$ increments between 1.5 and 


\section{MARBE Source Waveform}

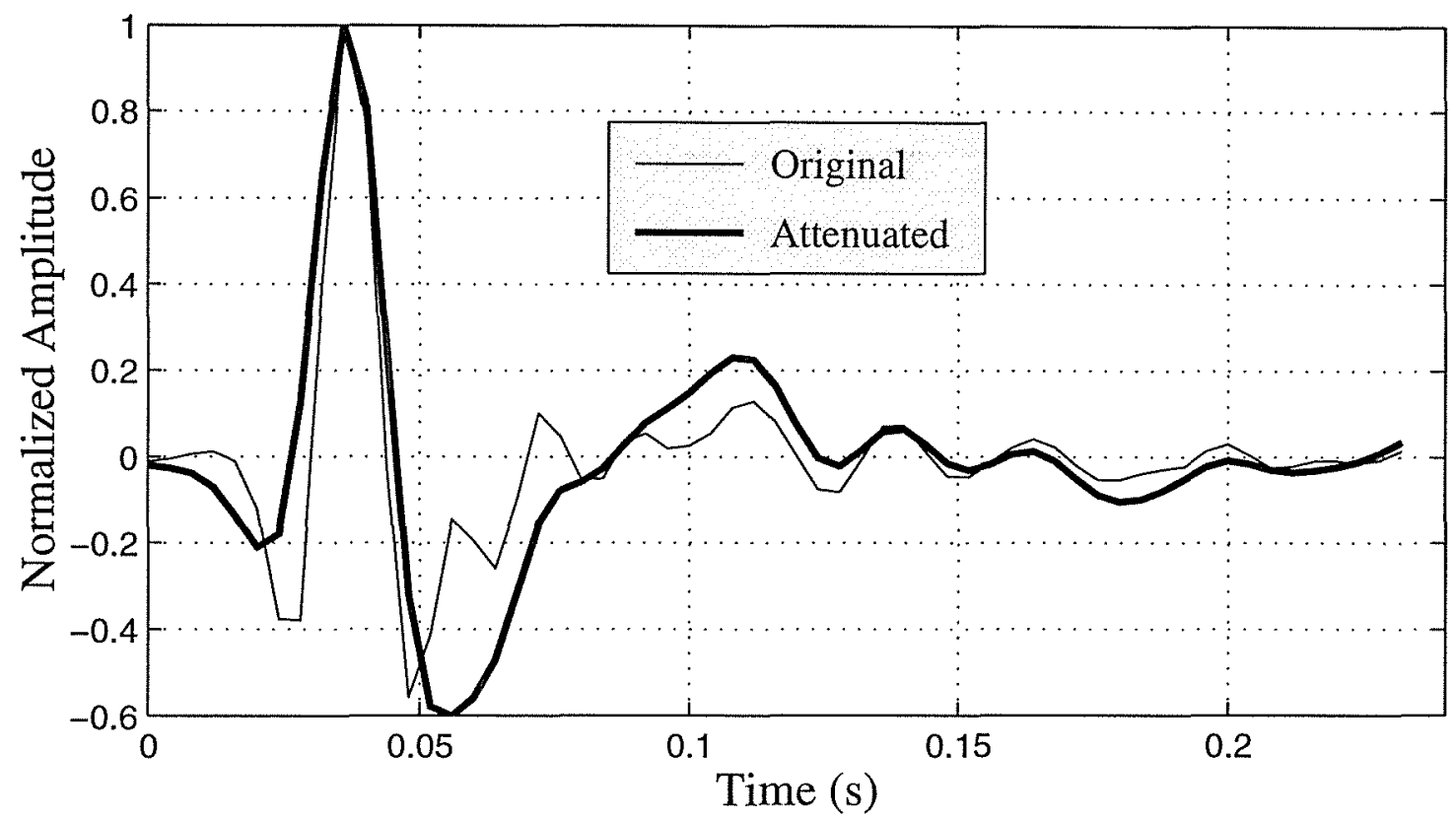

Figure 6-5: Original and attenuated source waveform used by the GA to analyze data from the MARBE survey. The original waveform was constructed by appropriately shifting and summing the near vertical incidence seafloor reflections along several sections of the survey (black lines or symbols in Figure 6-1). The effect of traveling twice through a typical layer $2 \mathrm{~A}\left(h=300 \mathrm{~m}, V_{p}=2.5 \mathrm{~km} / \mathrm{s}, Q=15\right.$ in this case) is contained in the attenuated waveform.

$2.1 \mathrm{~km} / \mathrm{s}$ on constant velocity stacks, and by consulting the GA supplied velocity functions. This velocity field was then used to construct the seismic profiles. Final stacks include information from only a limited set of ranges: $1.2-3.6 \mathrm{~km}$ for line $\mathrm{G}$, 0.8-3.2 for line H, 0.6-3.0 km for line I, and 1.0-3.4 for line L. Near-offset traces were omitted to enhance the amplitudes of refracted events relative to the seafloor and other reflections. Large ranges were not included because the nonhyperbolic moveout of upper crustal refractions turning within the high-velocity gradient tends to excessively distort far-offset energy during the stacking process [Harding et al., 1993; Vera and Diebold, 1994]. Final stacks were time migrated with the $45^{\circ}$ finite difference algorithm of Brysk [1983], which was chosen for its ability to handle steep dips and lateral velocity variations. 


\subsection{Results}

Upper crustal refractions were recorded and identified at all of the selected locations. For a detailed discussion on the geometry and origin of refracted energy turning within the upper crust, see Chapter 5 . The shoaling of the inner valley floor toward the center of OH-1 (Fig. 6-2) allows us to record upper crustal refractions in this area. A high-amplitude retrograde " $2 \mathrm{~A}$ " event, representing energy that turned within a steep velocity gradient, was clearly recorded in the two supergathers chosen from this area (Fig. 6-3a,b). Prograde "2B" energy from beneath this transition emerges from the seafloor reflection only at the farthest offsets. The successively elevated terraces east of the rift valley allow more refractions to fall within the $4 \mathrm{~km}$ aperture of the streamer. Line $\mathrm{H}$ supergathers from the intermediate terrace record shallower layer $2 \mathrm{~A}$ refractions prior to the seafloor reflection and capture more $2 \mathrm{~B}$ energy than their rift valley counterparts (Fig. 6-3 c,d). Line I is sufficiently shallow to allow the majority of upper crustal refractions to be recorded in front of the seafloor event (Figs. 6-3e,f), including prograde energy from immediately beneath the seafloor- "2ap" (Fig. 6-3e). In addition, two distinct retrograde $2 \mathrm{~A}$ branches were identified in supergather $\mathrm{I}$, and a $2 \mathrm{~A}$ multiple was recorded in supergather $\mathrm{I} 2$. West of the rift valley, the deepening of line $\mathrm{L}$ to the north is manifested in the successive delay of $2 \mathrm{~A}$ energy relative to the seafloor event (Fig. 6-4). No refractions were identified behind the seafloor reflection in the northernmost supergather (Fig.6-4e). All identified refractions were modeled during the GA analysis, the results of which are summarized in Table 6.2.

\subsubsection{Velocity Models}

The two study sites along line G are located $3.25 \mathrm{~km}$ apart, and lie within a smooth section of seafloor midway between the median valley ridge and the first bounding fault to the east (Figs. 6-1 \& 6-2). This region is documented by Bideau et al. [1996] as being the currently active volcanic depocenter. According to the inversion results, 
Table 6.2: Summary of Inversion Results

\begin{tabular}{|c|c|c|c|c|c|c|c|}
\hline \multirow{2}{*}{$\begin{array}{l}\text { Location } \\
\text { ID }\end{array}$} & \multirow{2}{*}{$\begin{array}{l}\text { Age } \\
\text { (Myr) }\end{array}$} & \multicolumn{3}{|c|}{ Layer 2A Thickness (m) } & \multirow{2}{*}{$\begin{array}{c}\text { Extrusive } \\
\text { Velocity* } \\
(\mathrm{km} / \mathrm{s})\end{array}$} & \multirow{2}{*}{$\begin{array}{c}\text { Transition } \\
\text { Zone Gradient } \\
\left(\mathrm{s}^{-1}\right)\end{array}$} & \multirow{2}{*}{$\begin{array}{c}\text { Depth to } \\
6 \mathrm{~km} / \mathrm{s} \\
(\mathrm{m})\end{array}$} \\
\hline & & $\begin{array}{l}\text { Low-Velocity } \\
\text { Interval }\end{array}$ & $\begin{array}{c}\text { Transition } \\
\text { Zone }\end{array}$ & Total & & & \\
\hline G1 & 0.0 & 161 & 417 & 578 & 2.31 & 6 & $?$ \\
\hline G2 & 0.0 & 181 & 228 & 409 & 2.31 & 11 & $?$ \\
\hline Mean & & 171 & 323 & 494 & 2.31 & 8 & \\
\hline H1 & 0.7 & 377 & 74 & 451 & 2.71 & 19 & 530 \\
\hline $\mathrm{H} 2$ & 0.7 & 239 & 73 & $\underline{312}$ & 2.64 & 31 & $\underline{395}$ \\
\hline Mean & & 308 & 74 & 382 & 2.68 & 25 & 463 \\
\hline I1 & 1.6 & 291 & 456 & 747 & 3.11 & 4 & $?$ \\
\hline $\mathrm{I} 2$ & 1.6 & $\underline{238}$ & $\underline{235}$ & $\underline{473}$ & 2.54 & 9 & $?$ \\
\hline Mean & & 265 & 346 & 610 & 2.83 & 6 & \\
\hline L1 & 1.9 & 325 & 26 & 351 & 3.00 & 44 & ? \\
\hline L2 & 1.9 & 311 & 39 & 350 & 2.66 & 38 & $?$ \\
\hline L3 & 1.9 & 67 & 218 & 285 & 2.42 & 10 & $?$ \\
\hline L4 & 1.9 & 396 & 32 & 428 & 2.82 & 58 & ? \\
\hline L5 & 1.9 & 257 & $\underline{250}$ & $\underline{507}$ & 2.81 & $\underline{8}$ & $?$ \\
\hline Mean & & 271 & 113 & 384 & 2.74 & 32 & \\
\hline
\end{tabular}

${ }^{*}$ Upper 50 meters 
both locations exhibit a thick $(160-180 \mathrm{~m})$, low-velocity $(2.3 \mathrm{~km} / \mathrm{s})$ surficial layer overlying an even thicker transition to layer 2B velocities (Fig. 6-6). However, from south (G1) to north (G2), the transition zone thins by nearly half, thereby raising the velocity gradient over this interval significantly (Table 6.2). We define layer $2 \mathrm{~A}$ as both the low-velocity layer and the transition zone. Our calculations therefore yield an on-axis layer $2 \mathrm{~A}$ thickness of 580 and $410 \mathrm{~m}$, for locations $\mathrm{G} 1$ and $\mathrm{G} 2$, respectively. A detailed examination of the inversion results for supergather G2 (Fig. 6-7) shows that the best velocity model fits the data quite well in both waveform and travel time ( $\phi=17.2$, see Appendix A.2). Note that, according to the Hydrosweep centerbeam data and seismic amplitudes, the true seafloor reflection arrives after a strong out-ofplane arrival. Although energy turning within the surficial low-velocity interval is not directly recorded, its thickness and velocity are constrained by the location and slope of the $2 \mathrm{~A}$ event, especially at large offsets. The data preclude seafloor velocities above $3 \mathrm{~km} / \mathrm{s}$, since energy turning within the uppermost crust would then be a first arrival (as in Fig. 6-3e). The minimum range to which the retrograde $2 \mathrm{~A}$ branch retreats is determined by the severity of the underlying transition zone. In supergather G2, a relatively weak velocity gradient places the caustic at a large range of $\sim 2.3 \mathrm{~km}$. This effect is more pronounced at site G1, where the gradient is even more subdued (Fig. 6-3a). Finally, the location and slope of the 2B branch record information on the velocity and depth of material below the transition zone. Although little of it precedes the seafloor reflection, the $2 \mathrm{~B}$ branch is sufficient to constrain the shallow properties of this layer.

On the eastern terrace (line $\mathrm{H}$ ), one study location is adjacent to a cluster of small seamounts, while the second is $2 \mathrm{~km}$ southeast of the platform edge, near the inside corner high. Both exhibit extremely high velocities $(>6 \mathrm{~km} / \mathrm{s})$ within a few hundred meters of the seafloor (Fig. 6-8). Basement velocities are slightly higher $(2.6 \mathrm{~km} / \mathrm{s})$, and thicknesses of the low-velocity layer are significantly greater (380 and $240 \mathrm{~m}$ thick, respectively) than on-axis. The transition zones of both span over 2.5 


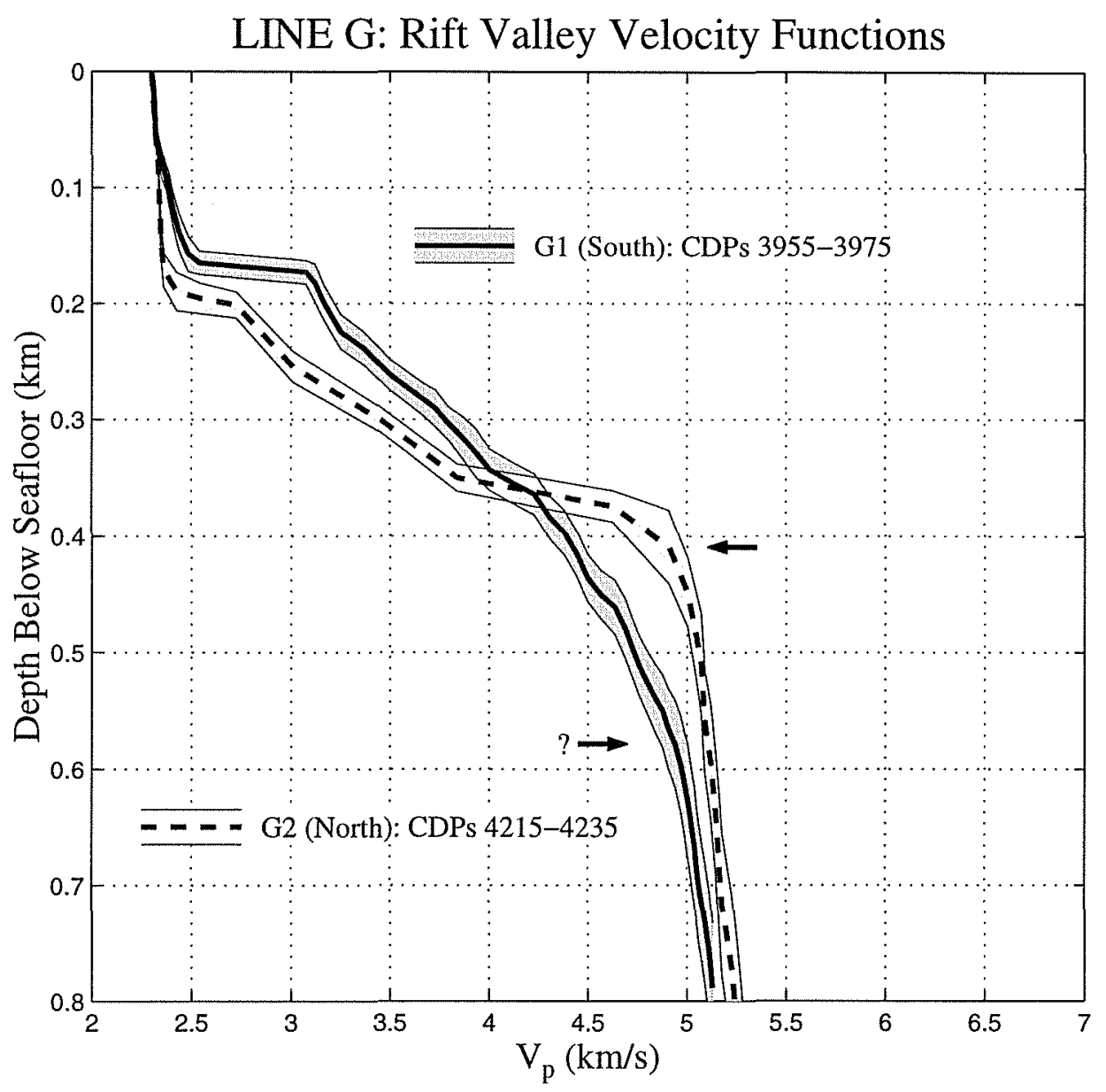

Figure 6-6: Mean final velocity models for the two study sites along on-axis line G. Structure deeper than $800 \mathrm{~m}$ was generally not modeled in this study. Grey shading delineates the one standard deviation error bound. These models show a $\sim 200-\mathrm{m}$-thick low-velocity layer overlying a transition zone of comparable or greater thickness. The base of layer $2 \mathrm{~A}$ (i.e., gradient) is picked by eye, and marked by an arrow along each curve. The gradual transition in the G2 velocity model makes the location of this horizon difficult ("?").

$\mathrm{km} / \mathrm{s}$ within an $80 \mathrm{~m}$ depth interval, but only the northern location (H1) suggests the existence of a layer with intermediate $(\sim 5 \mathrm{~km} / \mathrm{s})$ velocities. Evidence for this thin band of low-gradient material can be seen in the data at $2.3 \mathrm{~km}$ range, where an abrupt change in the slope and amplitude of the $2 \mathrm{~A}$ branch indicates the presence of a small triplication (Fig. 6-9). Although the data multiple was not used in the inversion, the final model's ability to locate the $2 \mathrm{~A}$ multiple $(2.5-2.8 \mathrm{~km}$ range) verifies its accuracy. A comparison of waveforms between the best model and the data shows 

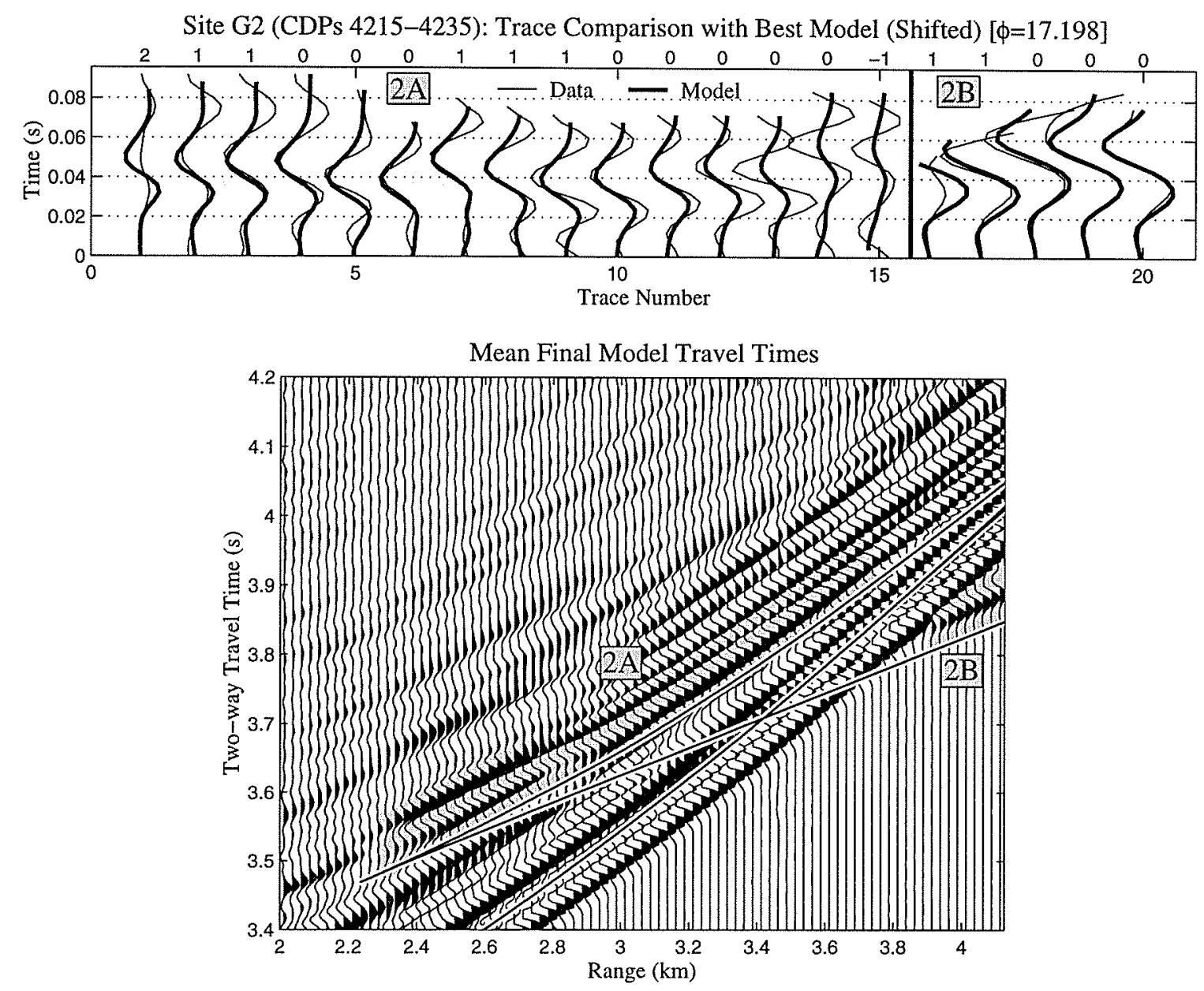

Figure 6-7: GA inversion results for on-axis supergather G2. (top) Waveform comparison between the best fitting model (thick) and data traces (thin). A vertical bar separates trace windows. To maintain computational efficiency, every fourth trace of the $2 \mathrm{~A}$ event and every second trace of the $2 \mathrm{~B}$ event was used to calculate the misfit. The row of integers corresponds to the number of samples each synthetic trace was shifted to achieve optimal fit, $\phi$. (bottom) Travel time curves for the mean final model in Figure 6-6, superimposed on the data seismograms. Unlike Figure 6-3, arrivals preceding and following the seafloor event are plotted at the same scale. No multiples were included in the forward models. Windows enclosing the selected refractions are shaded grey and labeled accordingly.

the close match between them, especially along the 2B branch (Fig. 6-9). Extremely high velocities near the seafloor are responsible for the shallow slope of this event at both study sites.

Both study locations along line I flank Eulália volcano (Fig. 6-1), but as is evident from their corresponding supergathers (Fig. 6-3e,f), they exhibit remarkably different 


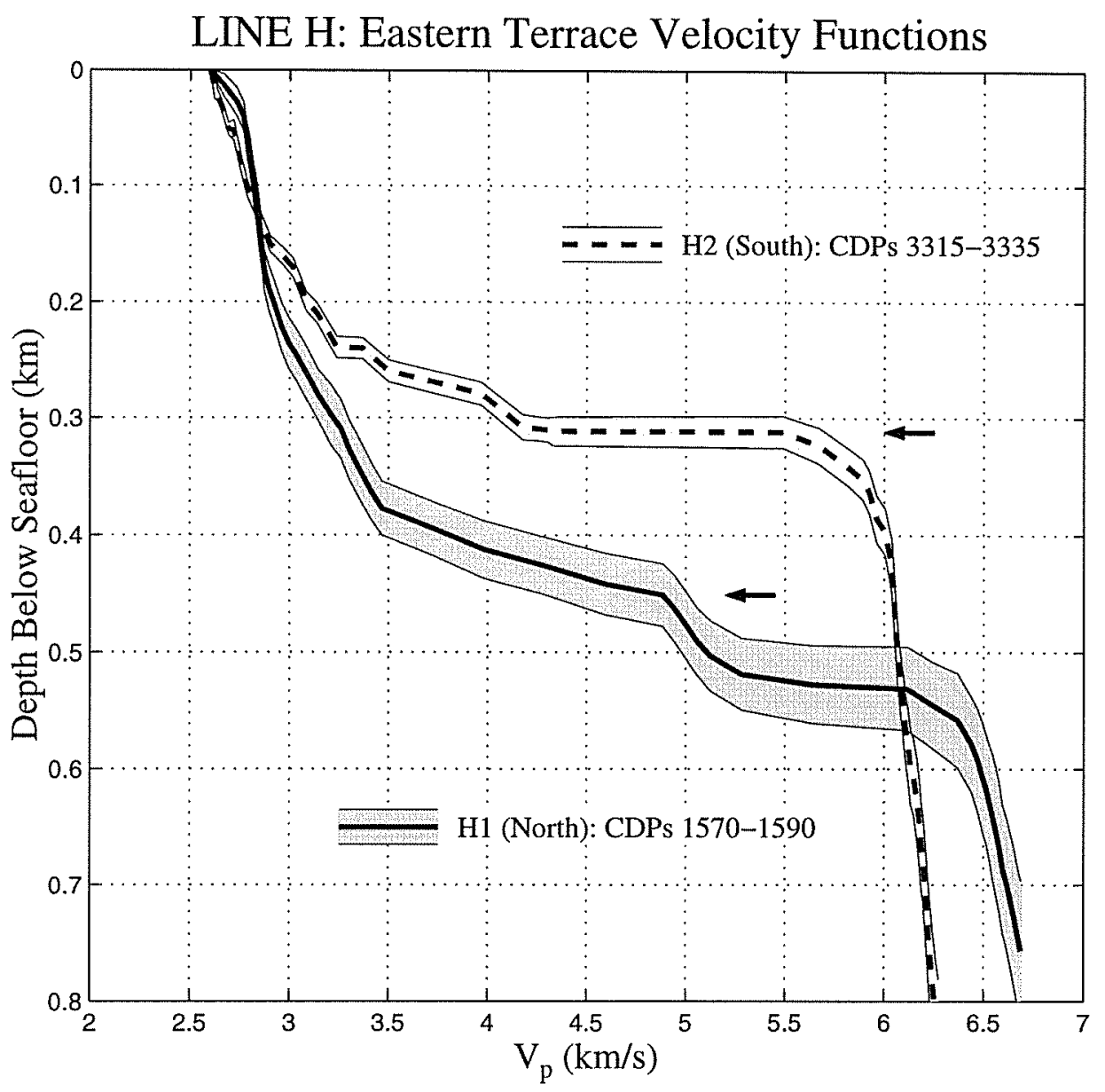

Figure 6-8: Same as Figure 6-6, except for the two study sites along eastern terrace line $\mathrm{H}$. Both models exhibit a transition from extrusive velocities $(<3 \mathrm{~km} / \mathrm{s})$ to values $>6 \mathrm{~km} / \mathrm{s}$ within a few hundred meters of the seafloor. An interval of layer $2 \mathrm{~B}$ velocities is found at $\mathrm{H} 1$, but is quite thin.

seismic structures (Fig. 6-10). Whereas the transition zone on the northern flank lies beneath a $240-\mathrm{m}$-thick low-velocity $(<3 \mathrm{~km} / \mathrm{s})$ layer and exhibits a weak undulation in velocity gradient, it is distinctly double-stepped beneath a slightly thicker, higher velocity layer $(>3 \mathrm{~km} / \mathrm{s})$ on the southern flank. Both models reach comparable layer 2B velocities at depth (470 and $750 \mathrm{~m}$, respectively), and contain a thickened, seismically faster surficial layer than on-axis. The first refracted arrival ("2ap") at location $\mathrm{I} 1$ is tangent to the seafloor reflection, and therefore records energy that has turned in the shallowmost crust (Fig. 6-11). Its slope is a direct measurement of the 

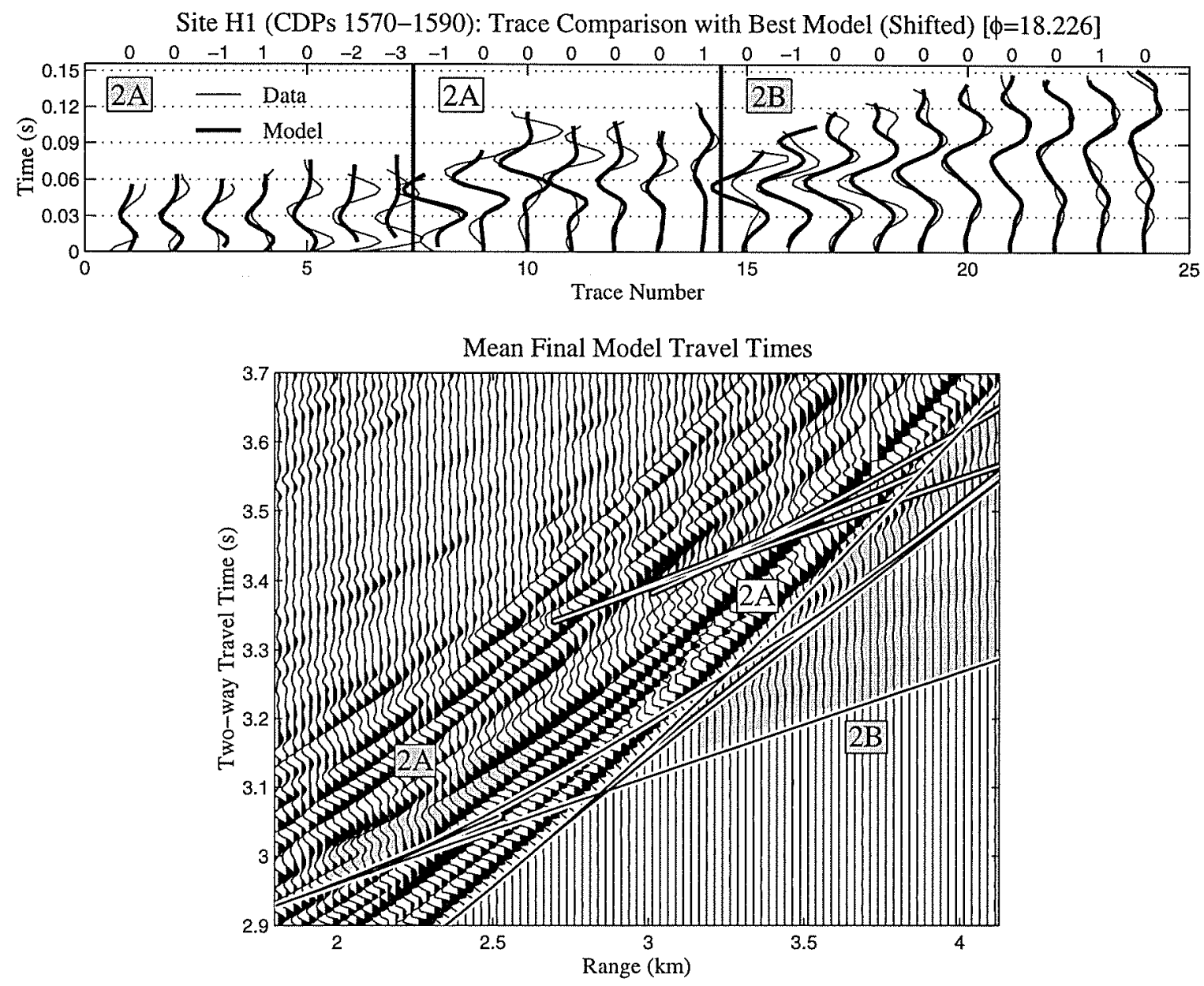

Figure 6-9: Same as Figure 6-7, except for eastern terrace supergather H1. Every fourth trace (including the $2 \mathrm{~B}$ event) was used to calculate the misfit. Synthetics did include multiples, which are shown as white-in-black curves on the travel time plot.

seafloor velocity, yielding a value of $3.1 \mathrm{~km} / \mathrm{s}$. Since velocity generally increases with depth, it is geologically reasonable that no high-slope refracted phases are observed, and that the majority of energy is recorded well in front of the seafloor reflection. The double-step structure at this location is constrained by the split $2 \mathrm{~A}$ branch located behind the seafloor reflection and by the bright triplication amplitudes at $3.1-3.4 \mathrm{~km}$ range. However, the complicated seismic pattern that is generated by the overlap of several refracted phases within a short time interval makes an accurate determination of the transition zone structure difficult (Fig. 6-10). The fact that the travel times of the mean final model do not extend back to the two windowed caustics observed in the 


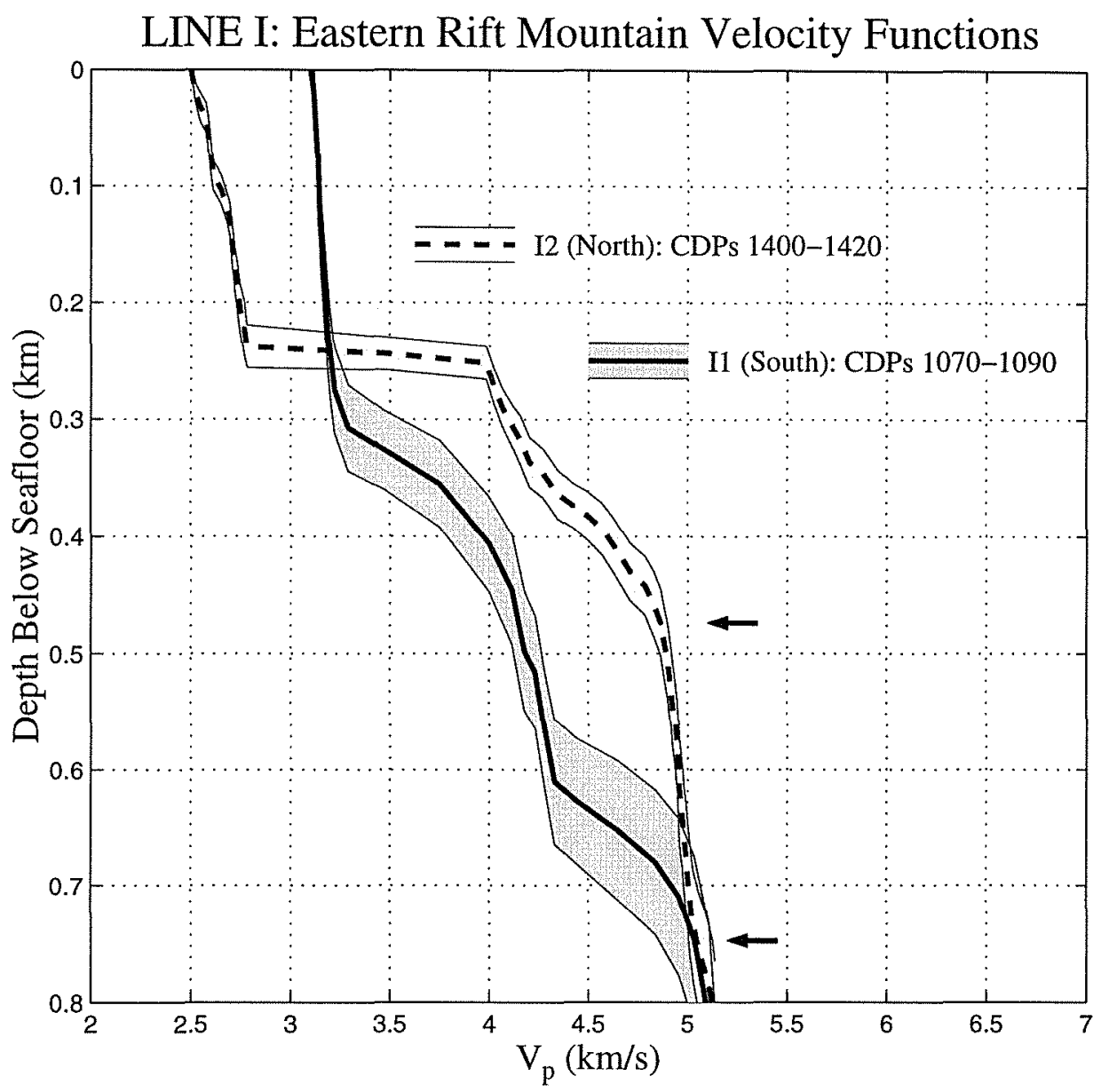

Figure 6-10: Same as Figure 6-8, except for the two study sites along eastern rift mountain line I. Whereas I2 displays a single-step transition to layer $2 \mathrm{~B}$ velocities, I1 exhibits a wider, two-step transition. Note, however, the greater uncertainty associated with the latter.

data (Fig. 6-11) indicates that the two high-gradient intervals may be sharper than predicted. Keep in mind that the mean final model is a weighted sum of all trials during a GA simulation [Ch. 5], and may therefore be a smoother function than any of the individual models used to construct it. Regardless of specific structure, however, the delayed $2 \mathrm{~B}$ arrivals alone prescribe the existence of an extremely thick transition zone at site I1. The early arrival of layer 2B energy at site I2 (Fig. 6-3f), and the existence of the $2 \mathrm{~A}$ caustic (and its multiple) at close range, constrain layer $2 \mathrm{~A}$, including the transition zone, to be thinner than at I1.

Line $\mathrm{L}$, shot along a ridge in the western rift mountains, contains five closely spaced 
Site I1 (CDPs 1070-1090): Trace Comparison with Best Model (Shifted) [ $\phi=24.055]$
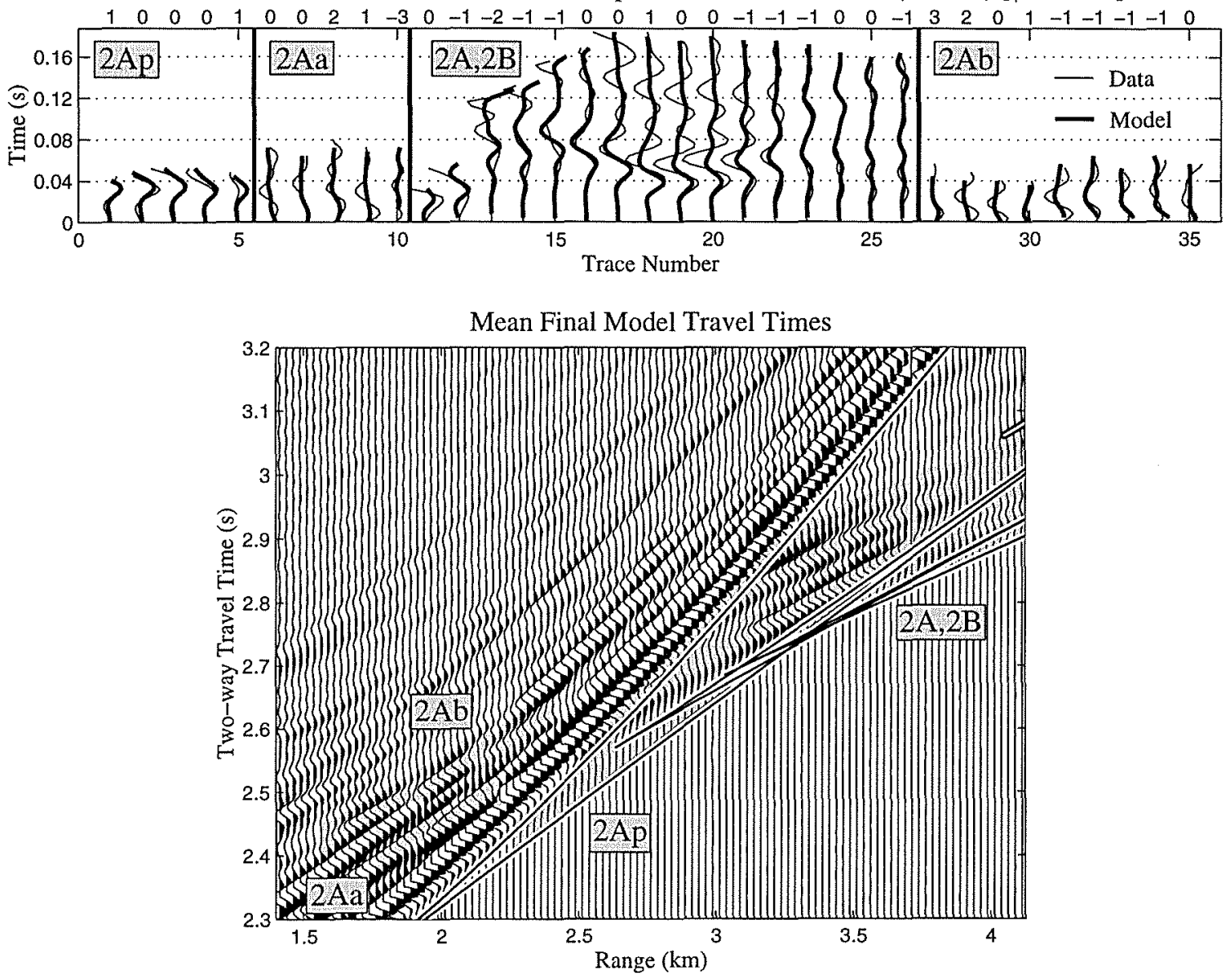

Figure 6-11: Same as Figure 6-9, except for eastern rift mountain supergather I1. Every fourth trace was used to calculate the misfit, which is significantly higher than at other locations. Multiples were included in the synthetics calculations, and are shown entering the lower panel at the far right. The bulk of their travel times, however, fall outside the streamer aperture.

study sites that descend toward the Oceanographer fracture zone in the north (Figs. 6-1 \& 6-2). Only $2 \mathrm{~km}$ separate the two southern locations (L1 and L2) which contain crust generated near the segment center and exhibit a very similar seismic character. Best fitting models for both areas consist of a 310-330 m thick low-velocity interval overlying an extremely sharp transition zone (Fig. 6-12). However, differences do exist within the uppermost crust. Not only does L1 exhibit a seafloor velocity 0.5 $\mathrm{km} / \mathrm{s}$ higher than at L2, it also contains a secondary structure near the base of the 


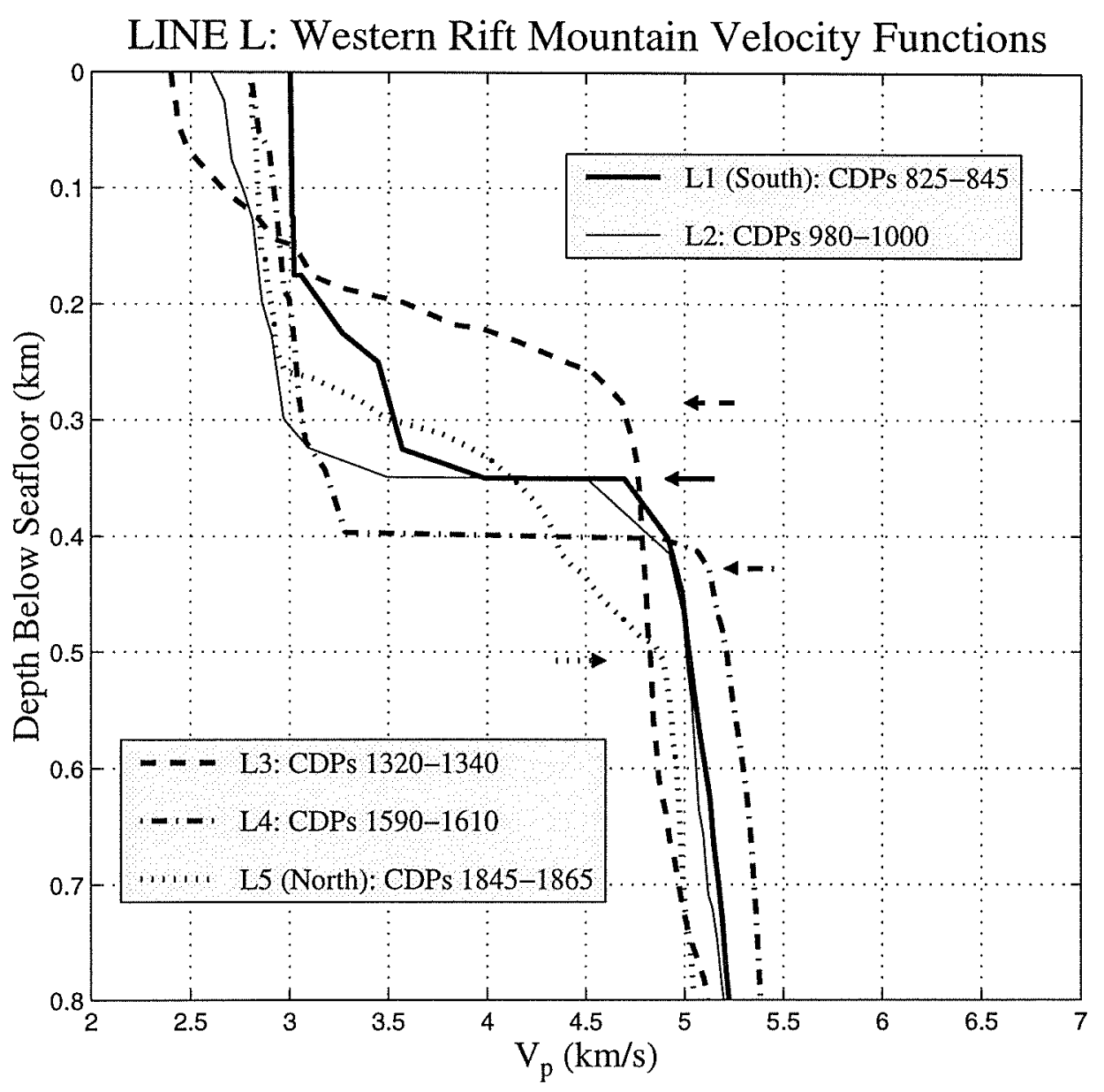

Figure 6-12: Same as Figure 6-10, except for the five study sites along western rift mountain line L. Error bounds are not shown, but are similar to those for the rift valley models. Each arrow matches the line pattern of its associated velocity function. The two most closely spaced sites, L1 \& L2, predict the same layer 2A thickness and 2B structure, but exhibit different velocities in the uppermost interval. From a velocity and thickness minimum at the center of the profile (L3), Layer $2 \mathrm{~A}$ thickens substantially to the north.

low-velocity interval. This thin, intermediate layer generates a small triplication that modifies the amplitudes of the $2 \mathrm{~A}$ event just in front of the seafloor reflection (Fig. 613). The small data misfit in Figure 6-13 exemplifies the excellent correlation between our final models and the data throughout line L. Sites L3 through L5 demonstrate a thickening of layer $2 \mathrm{~A}$ toward the inactive transform. It is thinnest at $\mathrm{L} 3$, where low velocities extend only $70 \mathrm{~m}$ below the seafloor before increasing to layer $2 \mathrm{~B}$ velocities at $280 \mathrm{~m}$ in depth. The $2.4 \mathrm{~km} / \mathrm{s}$ seafloor velocity of this area is comparable to that 
Site L1 (CDPs 825-845): Trace Comparison with Best Model (Shifted) [ $\phi=18.517]$
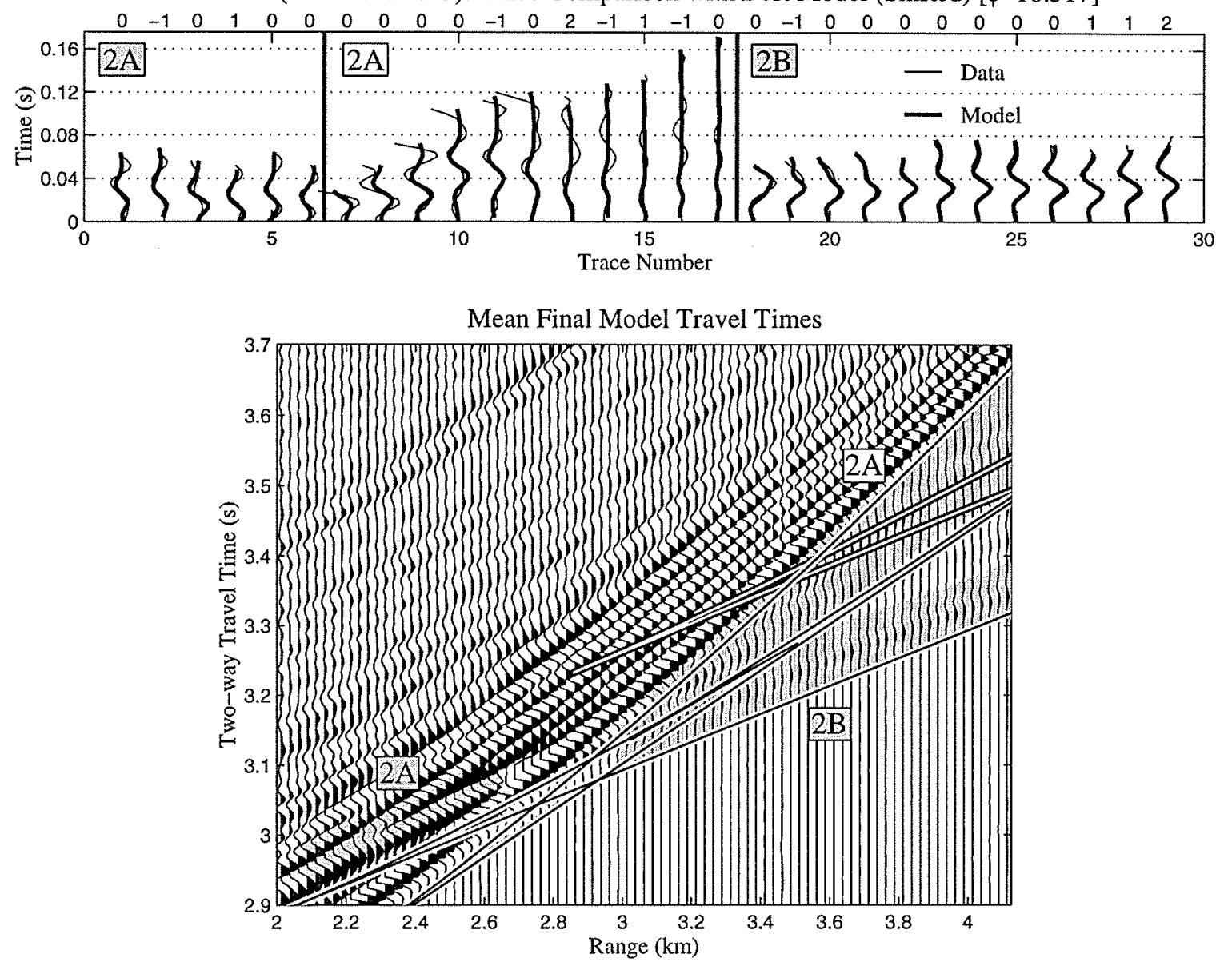

Figure 6-13: Same as Figure 6-11, except for western rift mountain supergather L1.

within the rift valley. Layer $2 \mathrm{~A}$ is thickest at L5, where the depth extent of the surficial low-velocity interval $(260 \mathrm{~m})$ and that of the transition zone $(250 \mathrm{~m})$ are nearly identical. Except for L3, every study location displays a thicker surficial layer and a steeper velocity transition than on-axis (Table 6.2).

\subsubsection{Seismic Profiles}

Conventionally processed CDP sections were generated along each of the four seismic lines. In every case, laterally continuous features were resolved on a scale of several km within the uppermost crust. This represents the first successful imaging of structure 
within the extrusive crust at the MAR [Detrick et al., 1990; Calvert, 1997; Smallwood and White, 1998]. The clarity and continuity of these seismic images were aided by the shallow water depths associated with the Azores hotspot and by the subdued along-isochron topography; seafloor slope rarely exceeds 5 degrees. The GA-deduced velocity models were converted to functions of two-way travel time (Fig. 6-14) and then placed within the seismic profiles to check for consistency between these methods and to correlate the imaged seismic horizons with specific velocity contrasts. The locations and optimal moveout velocities of these horizons were found to correspond well with those predicted by the individual models (Fig. 6-14).

Along the shallowest portion of the inner valley (Fig. 6-2), a continuous seismic horizon was imaged 0.2-0.4 s below the seafloor (Fig. 6-15a). This diffuse, longwavelength event spans up to $0.2 \mathrm{~s}$ in travel time and corresponds to the base of the wide transition zone predicted for both study sites. Moveout velocities match predicted values of $1700-1800 \mathrm{~m} / \mathrm{s}$ (Fig. 6-14). Except for a slight shoaling and focusing to the north near G2, this horizon exhibits no resolvable along-axis variations in either depth or frequency content.

In contrast to the rift valley, the eastern terrace exhibits a more complicated structure (Fig. 6-15b). Beneath H1, near the inside corner high, a diffuse band of coherent energy corresponds with the double-step transition to the $6.5 \mathrm{~km} / \mathrm{s}$ velocities observed in the GA results (Fig. 6-8). A deeper, stronger event merges with this horizon near CDP 1785, and continues shoaling to the south until it is lost in a short data gap. On the far side of this gap, an event is imaged just beneath the seafloor throughout the central portion of line H. Not until it reaches a cluster of seamounts to the south does it plunge well beneath the seafloor. The final models for $\mathrm{H} 1$ and $\mathrm{H} 2$ confirm that material beneath this horizon exhibits velocities above $6 \mathrm{~km} / \mathrm{s}$ at those locations. It is extremely unlikely that this event is the result of sideswipe from an out-of-plane diffractor. Not only do its optimal moveout velocities vary systematically with depth (1500-1800 m/s), but a probable candidate that would generate such a 

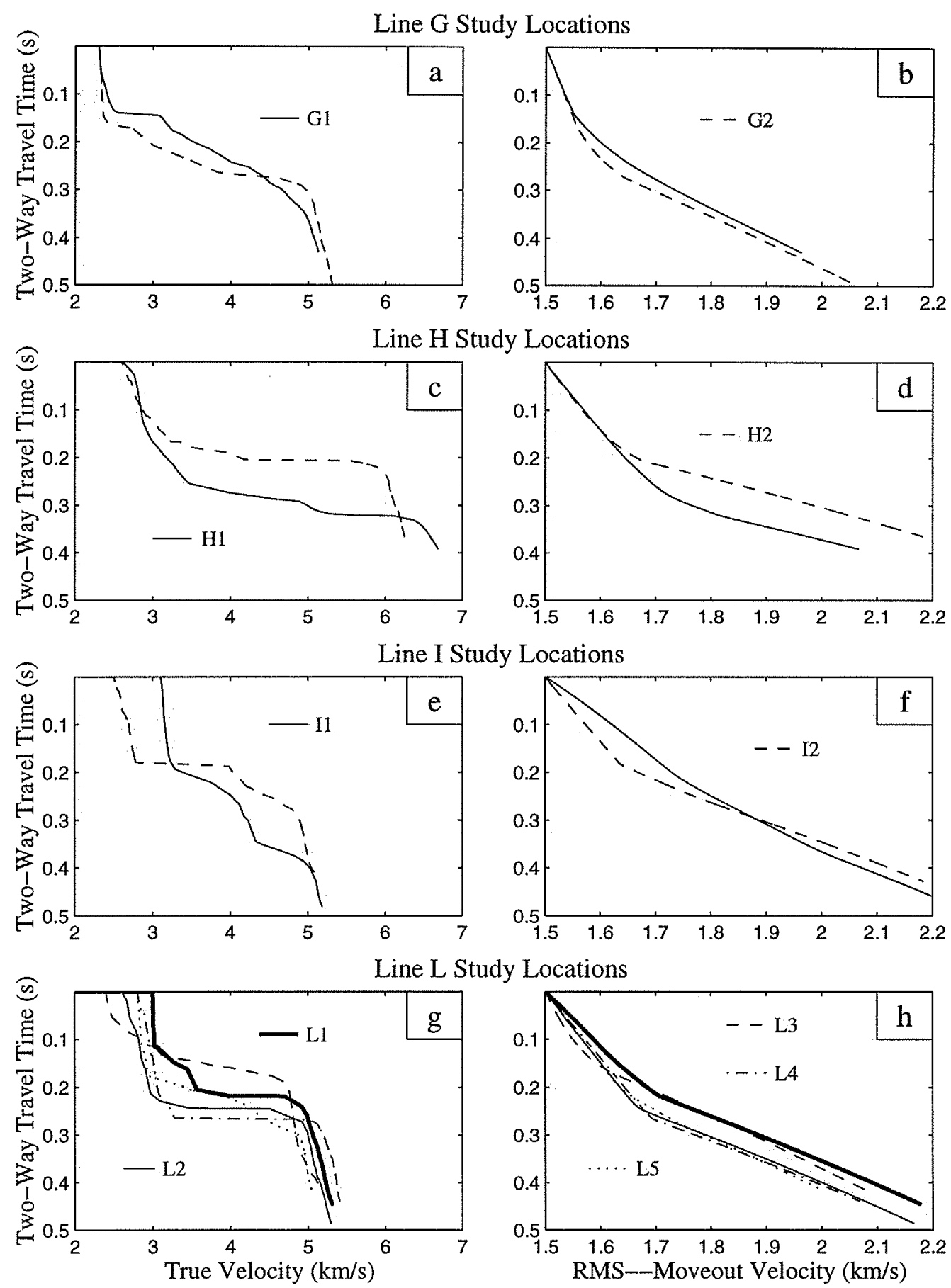

Figure 6-14: True velocity (a,c,e,g) and corresponding RMS, or moveout, velocity (b,d,f,h) for each study site. Both have been converted to functions of travel time. These model moveout velocities aid in the conventional processing of CDP data by providing benchmarks from which optimal stacking velocities can be found. The true velocities are useful in interpreting the lithostratigraphy of not only these discrete sites, but of an entire seismic line. 

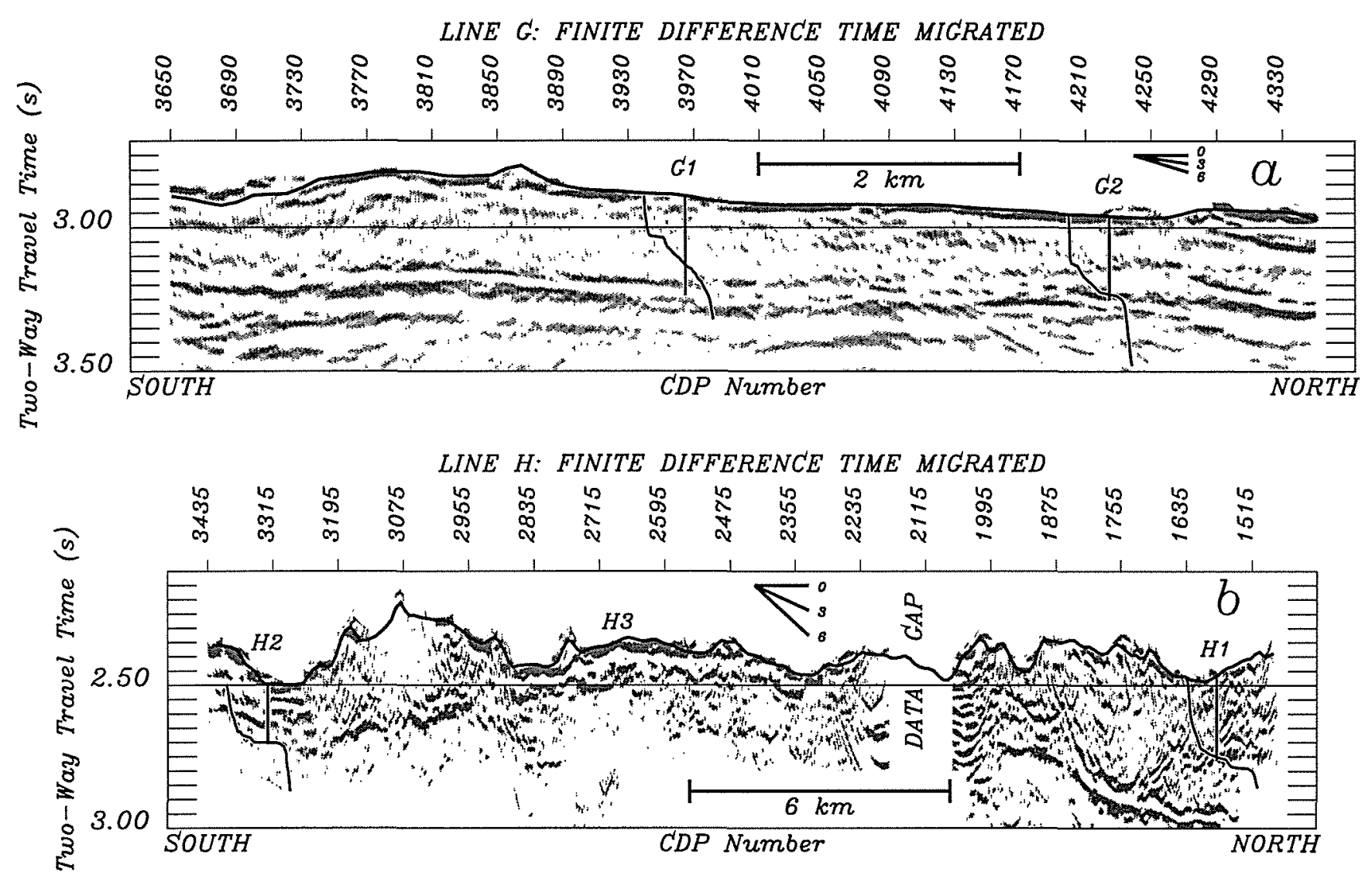

Figure 6-15: Stacked seismic sections along portions of a) on-axis line $G, b$ ) eastern terrace line $H, c)$ eastern rift mountain line $\mathrm{I}$, and d) western rift mountain line $\mathrm{L}$, the lateral extents of which are shown in Figure 6-2. These sections were migrated with a $45^{\circ}$ finite difference algorithm [Brysk, 1983], and include traces only between ranges of 1.2-3.6, 0.8-3.2, 0.6-3.0 and 1.0-3.4 km, respectively. Positive amplitudes are dark, and negatives are white. Bathymetry, as recorded by the centerbeam of coincident Hydrosweep data, is plotted on top of the seismically imaged seafloor, above which amplitudes are muted. Except for a short data gap in line $\mathrm{H}$, these panels represent continuous full-fold MCS sections, and contain all study sites. The GA-derived velocity functions are drawn at each location, with vertical bars representing the hand-picked layer $2 \mathrm{~A}$ thicknesses (Figs. 6-6, 6-8, 6-10 \& 6-12 ). Note the consistent agreement between these thickness estimates and the presence of a coherent seismic horizon. 

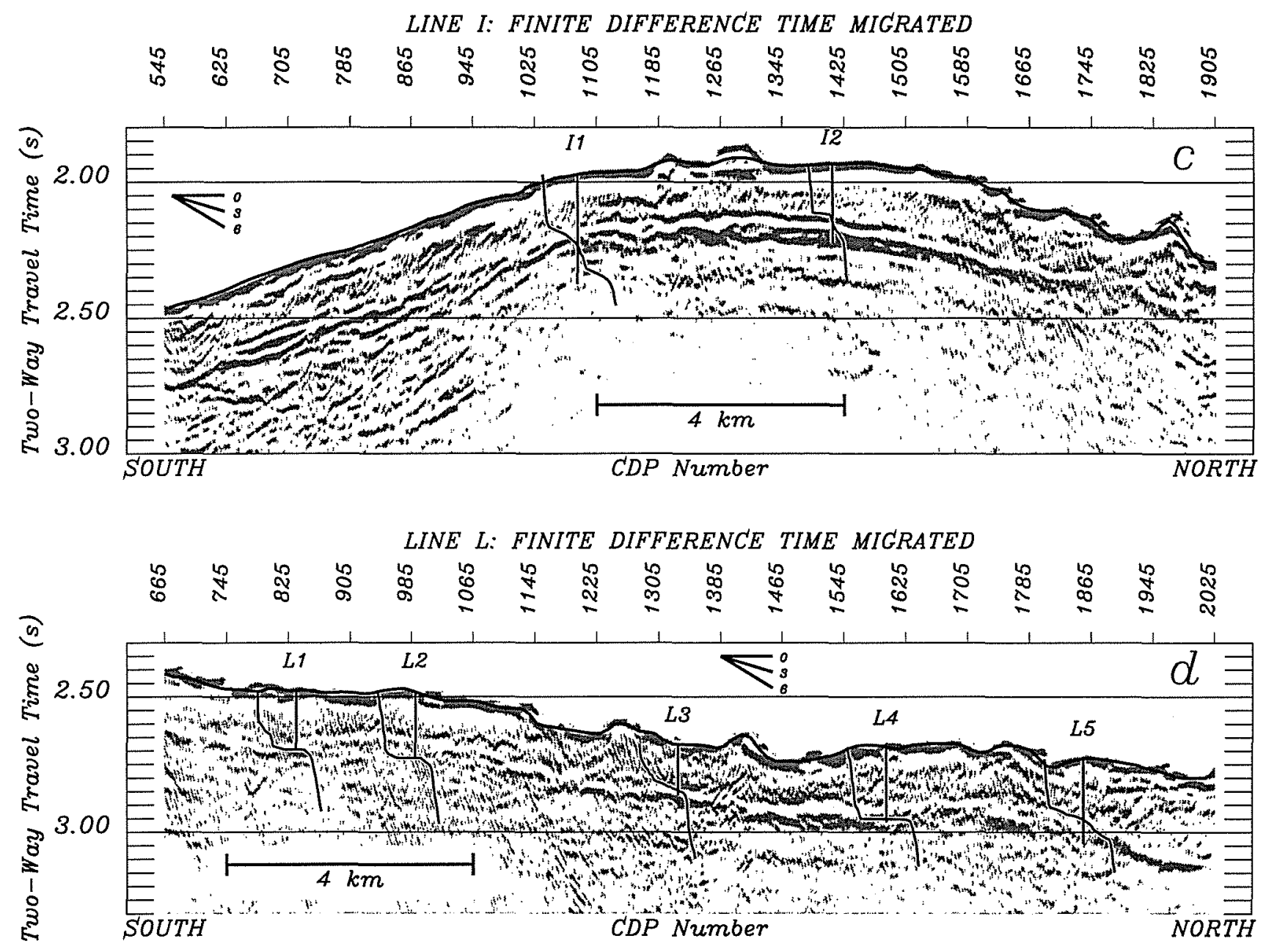

Figure 6-15: (continued) Along three of the lines (G, I \& L), the layer 2A event lies at a relatively uniform depth, but is less sharply defined on-axis $(G)$ and along the southern part of line I. The unusual subsurface reflectivity pattern of line $H$ is accompanied by extremely shallow high-velocity material. Site H3 was analyzed by hand. Relative to panel (a), vertical exaggeration increases threefold to (b), and twofold to (c) \& (d). Dip diagrams show a reference seafloor slope of $0^{\circ}, 3^{\circ}$, and $6^{\circ}$. 
continuous feature can not be found in the bathymetric data.

To test whether high-velocity material is also responsible for the shallow reflectivity pattern near the center of line $\mathrm{H}$ (Fig. 6-15b), we examined several supergathers from that area. Figure 6-16 shows that upper crustal energy in this region crosses the seafloor reflection at shot-receiver offsets of $<2 \mathrm{~km}$ - a full kilometer closer than observed elsewhere along the line. The slope of this event and its near tangency to

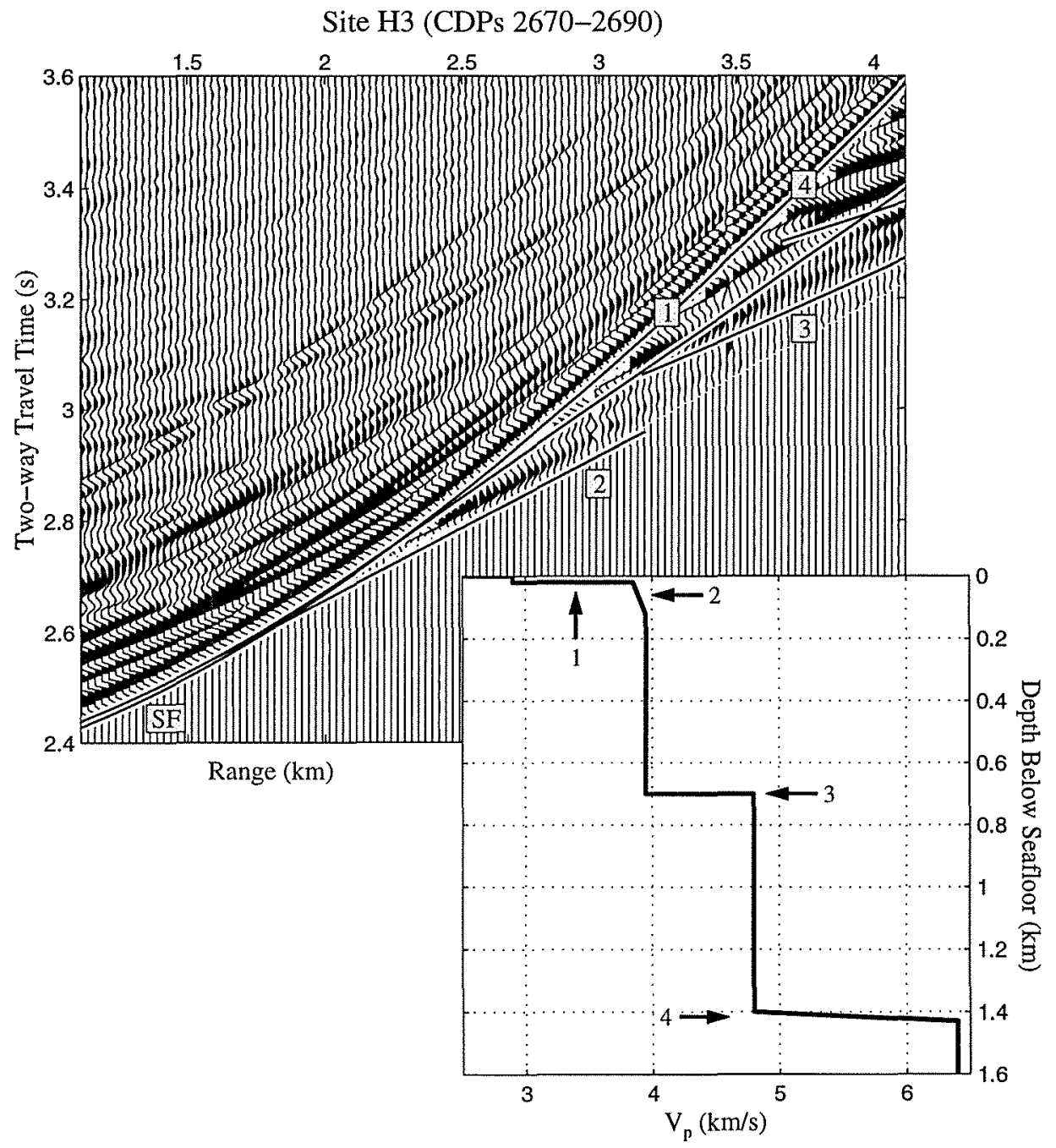

Figure 6-16: Travel time modeling results for the line $\mathrm{H}$ site marked by a star in Figure 6-15b. Upper panel shows the travel times of the hand-fit model plotted on top of the data seismograms. Numbered events correspond to velocity transitions in the best fitting velocity model (lower panel). Note the thick section of $4 \mathrm{~km} / \mathrm{s}$ material underlying an extremely thin $(20 \mathrm{~m})$ low-velocity layer, and the early first arrival it generates. 
the seafloor reflection are indicative of shallow, high-velocity material. Its sudden disappearance at large ranges signals the presence of a shadow zone, created by an underlying iso-velocity or negative gradient interval. Since the GA is prescribed to model only positive velocity gradients, it was not used to analyze this complicated structure. Instead, we hand-modeled the travel times of the four main seismic events that precede the seafloor reflection (Fig. 6-16). Our results show that, although velocities of nearly $4 \mathrm{~km} / \mathrm{s}$ are present beneath a 20 -m-thick veneer of low-velocity material, velocities above $6 \mathrm{~km} / \mathrm{s}$ are only found at depths greater than $1.4 \mathrm{~km}$. The deep transition to $6.4 \mathrm{~km} / \mathrm{s}$ material is constrained by the high-velocity event (\#4 in Fig. 6-16), which is considerably delayed in comparison to study sites $\mathrm{H} 1$ and H2 (Fig. 6-3c,d). Since this event lies entirely in front of the seafloor reflection, the inherent limitations of the normal moveout process prevent it from being imaged in the seismic profile (Fig. 6-15b). Similarly, the intermediate transition to $4.8 \mathrm{~km} / \mathrm{s}$ velocities is only weakly imaged at $0.3 \mathrm{~s}$ below the seafloor. If our interpretation is correct, then we have imaged a continuous seismic horizon underlain by $4 \mathrm{~km} / \mathrm{s}$ material in the center of the terrace and $>6 \mathrm{~km} / \mathrm{s}$ material near its northern and southern extremities.

Line I images a more uniform subseafloor structure than along the adjacent terrace. A single, continuous event $0.2-0.3 \mathrm{~s}$ beneath the seafloor clearly links the uppermost velocity gradient at site I1 to that of I2 (Fig. 6-15c). This event is most sharply defined directly beneath the seamount edifice (CDP 1285), and gradually thins to the north as it becomes weaker and more corrupted by the residual energy from a rougher seafloor. To the south, the event gradually thickens into a broad zone of coherent energy. As the thick transition interval of I1 indicates, the diffuse character of this event along the southern flank of Eulália seamount is the result of energy turning within a shallow velocity gradient over a large depth interval. In fact, this event is best focused by a $100 \mathrm{~m} / \mathrm{s}$ difference in moveout velocity from top to bottom (Fig. 6-14). 
The western rift mountain line L also displays a continuous seismic event, which connects the transition zones of all five study sites (Fig. 6-15d). Situated $\sim 0.2 \mathrm{~s}$ beneath the seafloor over a majority of the line, this horizon traces the depth of material with velocities of $3-4.8 \mathrm{~km} / \mathrm{s}$ over a lateral distance of $17 \mathrm{~km}$. The thinning of the uppermost layer near L3 is not apparent in the travel time section, since the depressed surficial velocities in this area (Fig. 6-12) counteract the effects of thinning. The predicted thickening of this layer to the north, however, is documented in the seismic profile, especially from CDP 1785 northward toward the fracture zone, where the upper crustal event plunges to $0.3 \mathrm{~s}$ below the seafloor. From a value of 1650 $\mathrm{m} / \mathrm{s}$ at site L3, the optimal stacking velocity for this horizon increases to 1825 and $1750 \mathrm{~m} / \mathrm{s}$ in the north and south, respectively. This progression of moveout velocities confirms the pattern of thinning and/or slowing of the surficial layer toward the center of the profile, and substantial thickening to the north (compare to Fig. 6-14). Similar to line $I$ in the east (Fig. 6-15c), this profile images a more focused upper crustal event (i.e., sharper transition zone) than does on-axis line G (Fig. 6-15a).

\subsection{Discussion}

Conventional CDP processing images a single seismic horizon within the upper kilometer of crust at segment $\mathrm{OH}-1$. This horizon is laterally continuous on a scale of tens of kilometers, and, with the exception of line $\mathrm{H}$, corresponds to a sharp velocity increase from $\leq 3$ to $\geq 5 \mathrm{~km} / \mathrm{s}$. Following the nomenclature established by Houtz and Ewing [1976] and adopted by subsequent studies at the EPR, we define the horizon imaged in the seismic sections, and resolved by the velocity analyses, as the base of layer $2 \mathrm{~A}$, which is underlain by layer $2 \mathrm{~B}$.

What are the geological properties responsible for generating this seismic horizon? As discussed in the previous chapter, porosity is the dominant factor controlling seismic velocities within the upper crust. The shallow crustal event documented both 
on- and off-axis is therefore most likely a porosity horizon, which may exist within the volcanic section as either a mechanical compaction boundary [Spudich and Orcutt, 1980], a hydrothermal precipitation or cracking front [Rohr et al., 1988; Wilkens et al., 1991] or a transition from rubbly to more massive lava flows [Harding et al., 1989]. However, it is difficult to convincingly associate the layer $2 \mathrm{~A} / 2 \mathrm{~B}$ transition with an intra-volcanic porosity boundary (see Christeson et al. [1996] \& Chapter 5). Our preferred model is that this horizon is controlled by the lithological transition from extrusives to sheeted dikes [e.g., Herron, 1982; Vera and Diebold, 1994; Christeson et al., 1996], with seismic velocity closely corresponding to the ratio of dike to extrusive material [Ch. 5]. This is especially true for the near-ridge environment, where hydrothermal processes have not yet modified the porosity structure significantly (e.g., Hole 504B [Becker et al., 1988]).

A comparison of the velocity functions obtained in this study with those from others provides useful information about the formation pattern of extrusive crust at different spreading rates (Fig. 6-17). On-axis, the analysis of Purdy and Detrick [1986] is the only other MAR study to provide detailed seismic constraints on the upper kilometer of crust. A comparison of our line $\mathrm{G}$ velocities with theirs yields a similar layer $2 \mathrm{~A}$ thickness, but different transition zone widths (Fig. 6-17a). The velocity discontinuity of Purdy and Detrick [1986], however, is not an indication of true geology, but is rather a consequence of modeling only arrivals that precede the seafloor event. Our results more closely match those from the Juan de Fuca Ridge [Cudrack and Clowes, 1993; McDonald et al., 1994] (Fig. 6-17a). The agreement of site G2 with the model of McDonald et al. [1994], in particular, suggests that the shallow zero-age structure of intermediate spreading crust is quite similar, on average, to that of slow spreading ridges. Both exhibit a thick $(\geq 200 \mathrm{~m})$ low-velocity interval within a layer $2 \mathrm{~A}$ that is $350 \mathrm{~m}$ thick or greater. With the exception of site L3, we resolve a consistently thicker surficial low-velocity layer off-axis than within the rift valley (Table 6.2). No such systematic transition to a distinct off-axis structure 

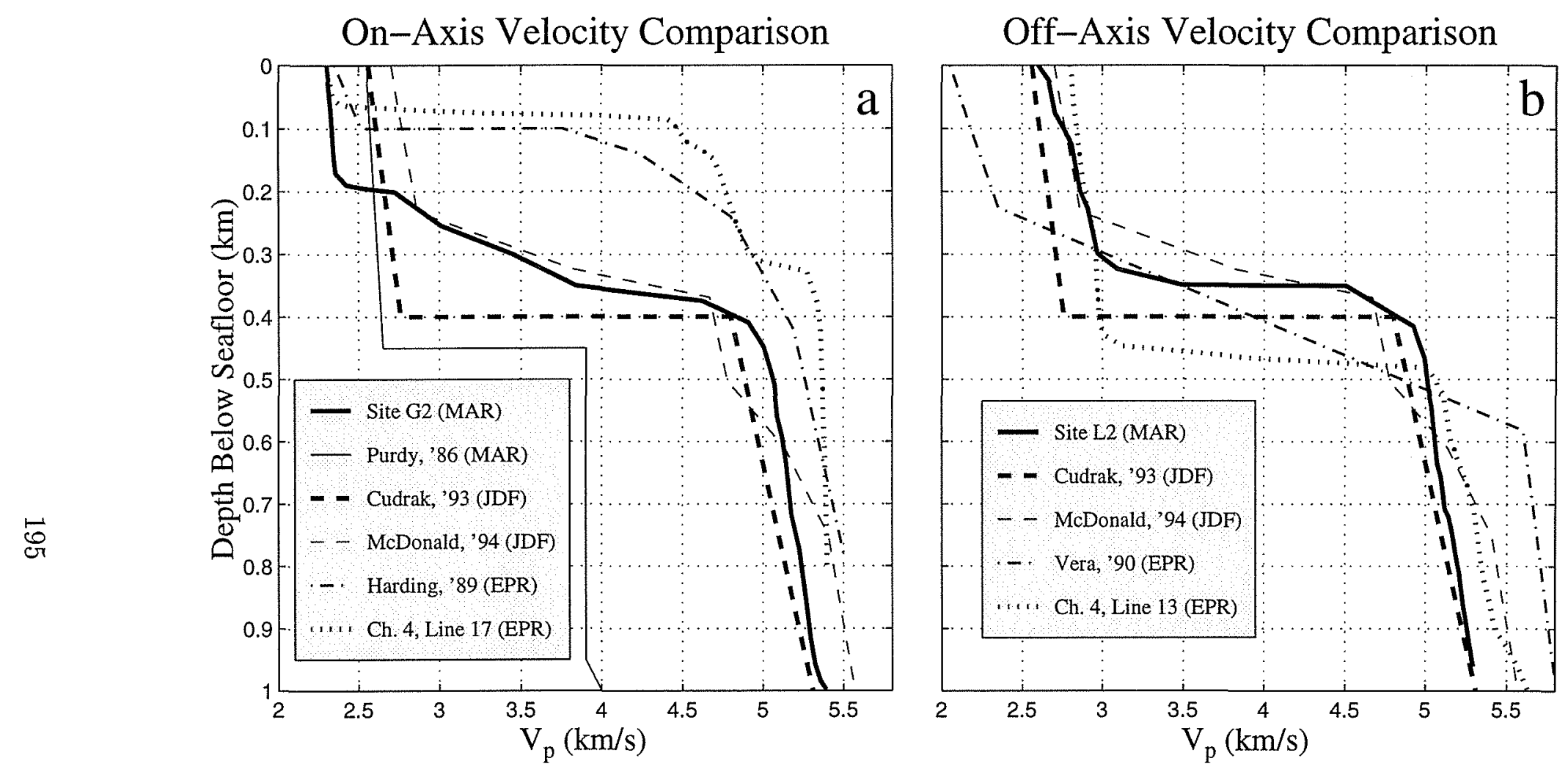

Figure 6-17: (a) On- and (b) off-axis comparison of upper crustal velocity structure between the superfast spreading southern East Pacific Rise (EPR), intermediate spreading Juan de Fuca Ridge (JDF), and slow spreading Mid-Atlantic Ridge (MAR). The only detailed model of the upper kilometer of MAR crust is that of [Purdy and Detrick, 1986]. The JDF models of Cudrack and Clowes [1993] and McDonald et al. [1994] are 1-D averages of their entire survey areas, and are therefore plotted in both panels. EPR models are from Harding et al. [1989], Vera et al. [1990] and Chapter 5 of this thesis (Line 17-Supergather 14 \& Line 13-Supergather 12). Note how the shallow transition zone at the EPR deepens off-axis to match the velocity structure observed at slower spreading rates more closely. 
was detected at the JDFR by Cudrack and Clowes [1993] or McDonald et al. [1994], and no other data from the MAR are available for comparison. In contrast to these ridges, the EPR displays a thin (60-120 m) on-axis interval of low velocities (Fig. 6-17a) that thickens by over $300 \%$ to either meet or exceed the off-axis value of all other spreading centers (Fig. 6-17b). Consequently, this comparison suggests that the off-axis structure of the upper crust is quite similar across a wide range of spreading rates, whereas a distinct on-axis difference exists between the fast spreading EPR and other, slower spreading ridges.

\subsubsection{Construction of the Upper Crust}

Because of its uniform character and low velocities (generally $<3 \mathrm{~km} / \mathrm{s}$ ), the surficial layer identified at all line G, I, and L study sites is likely to be entirely composed

of high-porosity extrusives. Such low velocities indicate that the bulk porosity of this layer may exceed $20 \%$, depending on the shape and distribution of void spaces [Spudich and Orcutt, 1980; Purdy, 1987; Wilkens et al., 1991; Berge et al., 1992; Jacobson, 1992]. If the underlying transition zone represents an increased dike fraction with depth, then our seismic measures (Table 6.2) provide constraints on the extrusive emplacement process at slow spreading ridges.

Seafloor observations [e.g., Ballard and Van Andel, 1977; Chadwick et al., 1995; Lawson et al., 1996] and kinematic models [Cann, 1974; Dewey and Kidd, 1977; Kidd, 1977; Pálmason, 1980; Hooft et al., 1996] have established that the bulk of extrusive crust is built by dike injection and lava extrusion within a neovolcanic zone at the spreading axis, be it within a summit trough (EPR) or along a median valley ridge (MAR). However, the lateral deposition of lava flows produces a zone of volcanic accretion that is wider than the off-axis extent of dike emplacement. The surficial layer of pure extrusives, therefore, represents the volume of lavas emplaced outside the zone of dike intrusion, while lavas that share the velocity transition with the dikes were emplaced within this zone. 
The surficial layer is generally thicker in the rift mountains than within the rift valley. This may indicate that a significant portion of the extrusive section is built outside the valley floor. The chain of seamounts extending off-axis from the segment center provides ample evidence for near-axis volcanism [Bideau et al., 1996]. However, the $25 \mathrm{~km}$ lateral extent of the thickened volcanic layer along line L contrasts sharply with the localized thickening of this layer near the seamounts along lines $\mathrm{H}$ and $\mathrm{I}$ (Fig. 6-15b,c), indicating that seamount volcanism alone is insufficient to explain the off-axis thickening of layer $2 \mathrm{~A}$.

Other factors are more likely to contribute to the observed thickness difference between lines $\mathrm{G}$ and $\mathrm{L}$. One argument is that extrusive thickness varies considerably within the rift valley, as suggested by Barclay et al. [1998]. If line L follows along a relict neovolcanic ridge (a past locus of basaltic construction) then the measured extrusive layer may be thicker than in adjacent seafloor that was created contemporaneously in the rift valley. Line $\mathrm{G}$ was acquired in the eastern trough of the median valley floor, and may therefore record a thinner extrusive section than at the constructional ridge or against the eastern bounding fault, where lavas may pond. Tectonic controls on volcanic emplacement are considerable [e.g., Macdonald and Luyendyk, 1977; Kappel and Ryan, 1986; Carbotte et al., 1997], and likely to be important factors in determining the volume of extrusives deposited on any given parcel of crust. Such a process is exemplified by the juvenile bounding fault that has bisected the constructional ridge and may be preventing further deposition of lavas in the western trough. An episodicity to bounding fault generation (see Macdonald and Luyendyk [1977] and Hussenoeder et al. [1996b]) would produce sections of crust that have spent different amounts of time in the rift valley, and hence exhibit different extrusive thicknesses. If uncharacteristically thick, the volcanic layer along line $\mathrm{L}$ may be part of a crustal block that experienced a lengthy residence within the inner valley floor. While these factors may significantly affect the final thickness of extrusives at a given location, it is difficult to ascertain their relative importance without further studies. It must 
be noted, however, that the primary process of cumulatively building a basaltic pile is still active in the eastern trough. It is reasonable to suggest that the extrusive crust there will simply continue to build until it is rafted out of the inner valley by a juvenile bounding fault, perhaps reaching a thickness comparable to that measured in the rift mountains.

Although line $\mathrm{G}$ clearly images a relatively constant thickness extrusive section along the rift valley floor (Fig. 6-15a), it does not measure the geometry of this layer across the inner valley. Cross-axis constraints for this area, albeit lower resolution, come from tomography results, which show that layer $2 \mathrm{~A}$ exhibits no systematic trends in thickness across the inner valley [Barclay et al., 1998]. A uniform extrusive thickness may indicate that the axis of volcanism is highly unstable within a wide zone of dike injection ( $r<2$, see Eq. 5.2), possibly encompassing the entire inner valley floor. However, such a model would predict an extremely thick transition zone and a thin or non-existent low-velocity layer (see Pálmason [1980] and Hooft et al. [1996]). The fact that, along line $\mathrm{G}$, nearly $200 \mathrm{~m}$ of pure extrusives overly a transition zone of comparable thickness to that at fast spreading ridges (Fig. 6-17a) indicates that a wide zone of lava deposition, not dike emplacement, is responsible for the thickness pattern we observe in the rift valley. Although quantifying the emplacement dynamics in this area is not possible with the limited information available to us, it is clear that a large portion of the inner valley floor lies outside the zone of dike injection, but within the zone of lava deposition. The absence of an observable off-axis thickening at the JDFR [Cudrack and Clowes, 1993; McDonald et al., 1994] suggests that volcanic accretion at intermediate spreading ridges (50-100 km/Myr full rate) may be quite similar to that at the MAR.

The morphologic similarity of the ridge beneath line $\mathrm{L}$ to those within the rift valley of slow spreading ridges indicates that it may be a volcanic constructional feature that has been rafted into the rift mountains relatively intact [D. K. Smith, personal communication, 1998]. Both seismic reflectivity (Fig. 6-15d) and velocity 
results (Fig. 6-12) along this line show evidence for a substantial deepening of the layer $2 \mathrm{~A}$ event toward the Oceanographer fracture zone. Although a tectonic thickening of layer $2 \mathrm{~A}$ due to enhanced fracturing near the inactive transform can not be ruled out, we see no reason for fracturing to cease abruptly enough within a lithologic unit to generate an observable seismic horizon. The deepening horizon more likely records a thickening volcanic layer. Yet, a larger volume of extrusives near the northern end of this segment is counter to models of focused magmatic accretion [e.g., Lin and Phipps Morgan, 1992], which predict a thicker crust and a more direct melt supply to the seafloor near the shallow segment center. Crustal thickness estimates from seismic refraction experiments [Sinha and Louden, 1983; Canales and Detrick, 1998; Hooft et al., 1997b; Hosford et al., 1998] and gravity measurements [Detrick et al., 1995], as well as submersible observations of the inner valley [Bideau et al., 1996], support a model of focused accretion. We therefore postulate that the thickening of layer $2 \mathrm{~A}$ toward the Oceanographer fracture zone along line $\mathrm{L}$ is the result of alongaxis transport of magma away from the segment center by lateral dike injection. Unfortunately, the deepening of the rift valley toward the transform prevented the limited aperture of the multichannel streamer from documenting a possible thickening of layer $2 \mathrm{~A}$ in crust that is currently forming. However, side-scan sonar imagery south of the Atlantis transform, find evidence that eruptive features near the transform were fed by a magma source closer to the segment center [Smith et al., 1998]. They postulate that this process may be controlled, in part, by the steep slope along the rift valley axis. Kilauea volcano, Hawaii is a paradigm of lateral melt transport along a centrally supplied rift zone [e.g., Tilling and Dvorak, 1993]. Here, rifting events have transported large volumes of melt downslope for distances over $100 \mathrm{~km}$, forming the submarine Puna Ridge. The average slope between the transform valley the center of $\mathrm{OH}-1$ is $52 \mathrm{~m} / \mathrm{km}$, steeper than most MAR segments, including that studied by Smith et al. [1998], and comparable to that of Puna Ridge. Based on the off-axis seismic evidence, we suggest that some subsurface redistribution of melt is occurring along 
the rift valley, which may thicken layer $2 \mathrm{~A}$ away from the segment center. This is in contrast to the EPR, where the subdued segment-scale bathymetry and presence of a nearly continuous magma sill reduce the likelihood that lavas erupted near segment offsets originate at their centers.

Rock magnetization [Johnson and Atwater, 1977; Bleil and Peterson, 1983] and magnetic anomaly studies [Blakely, 1983; Wittpenn et al., 1989] present strong evidence that the highly magnetic pillow basalts are the major contributor to marine magnetic anomalies in juvenile crust. If the extrusive section does carry the bulk of the crustal magnetic signature at young ages, then a thickening of layer $2 \mathrm{~A}$ may be partly responsible for the strengthening of the axial magnetic high toward the distal ends of this segment [A. H. Barclay; A. Hosford, unpublished data] and others along the MAR [e.g., Hussenoeder et al., 1996b; Weiland et al., 1995]. The cross-axis character of magnetic anomalies are often interpreted in terms of layer $2 \mathrm{~A}$ thickness variations [Tivey and Johnson, 1993; Gee and Kent, 1994; Schouten et al., 1998], but along-axis trends are typically associated with other factors, including lava chemistry [Vogt and Johnson, 1973; Christie and Sinton, 1981; Sempéré, 1991], thermal effects [Grindlay et al., 1992], serpentinite [Pockalny et al., 1995], and valley wall proximity [Hussenoeder et al., 1996b]. The northern end of line L suggests that layer $2 \mathrm{~A}$ thickness variations may also be important in determining the along-axis character of magnetic anomalies at the MAR.

\subsubsection{Maturation and Modification of the Upper Crust}

Table 6.2 documents an increase in velocity with age for the extrusive section. This

phenomenon has been observed at both fast [e.g., Grevemeyer and Weigel, 1997] and slow spreading ridges [Purdy, 1987], and is commonly attributed to the gradual cementation of small-scale porosity with hydrothermal precipitates [Houtz and Ewing, 1976; Wilkens et al., 1991; Jacobson, 1992]. By comparing our results to the velocityage relationship developed for young (<10 Myr-old), fast-spreading crust [Ch. 5], 
we find that the off-axis increase in extrusive velocities determined by this study is more subdued than at the EPR (Fig. 6-18). The curve that best fits the most recent observations at the MAR [Purdy and Detrick, 1986; Purdy, 1987; this study] in a least squares sense is defined by an error function [Grevemeyer and Weigel, 1996],

$$
V_{p}(t)=V_{0}+\alpha \int_{0}^{t} \exp \left(-\beta t^{2}\right) d t
$$

where the zero-age velocity $\left(V_{0}\right)$ is $2.35 \mathrm{~km} / \mathrm{s}$, and the two constants $(\alpha$ and $\beta$ ) are 0.26 and 0.002 , respectively. Unlike a $t^{\frac{1}{2}}$ function, which best fits the EPR data, this curve describes a nearly constant velocity increase in the first 10 Myr (Fig. 6-18).

Such a gradual change may indicate that hydrothermal processes are less vigorous and/or pervasive in the near-ridge environment at slow spreading rates. Hydrothermal vent fields are observed at the MAR, but are often localized by tectonic features [e.g., Kleinrock et al., 1996]. The lack of a detectable magmatic heat source in slow spreading environments [Detrick et al., 1990] also supports a model of decreased hydrothermal activity. The difference in velocity-age curves may also reflect the greater offset between apparent crustal age (from spreading rate) and the true age of extrusives at slow spreading ridges. Particularly if the neovolcanic zone spans the entire valley floor, which encompasses hundreds of kyrs of spreading history, the uppermost lavas will consistently be of a younger age than their off-axis location suggests, thereby flattening the velocity-age relationship. The paucity of high-quality data at the MAR, however, makes an accurate determination of the velocity-age function difficult. Clearly, additional studies are required to better constrain the age dependence of extrusive velocities at slow spreading ridges.

According to volcanic emplacement models [e.g., Hooft et al., 1996], the transition zone thickness may be used as a measure of focused accretion. The wider the zone of dike injection, the thicker the depth interval over which volcanics are permeated with higher velocity dike material. The thick transition zone measured in the rift valley (Figs. 6-6 \& 6-15a) indicates that the area of dike injection is fairly wide, 


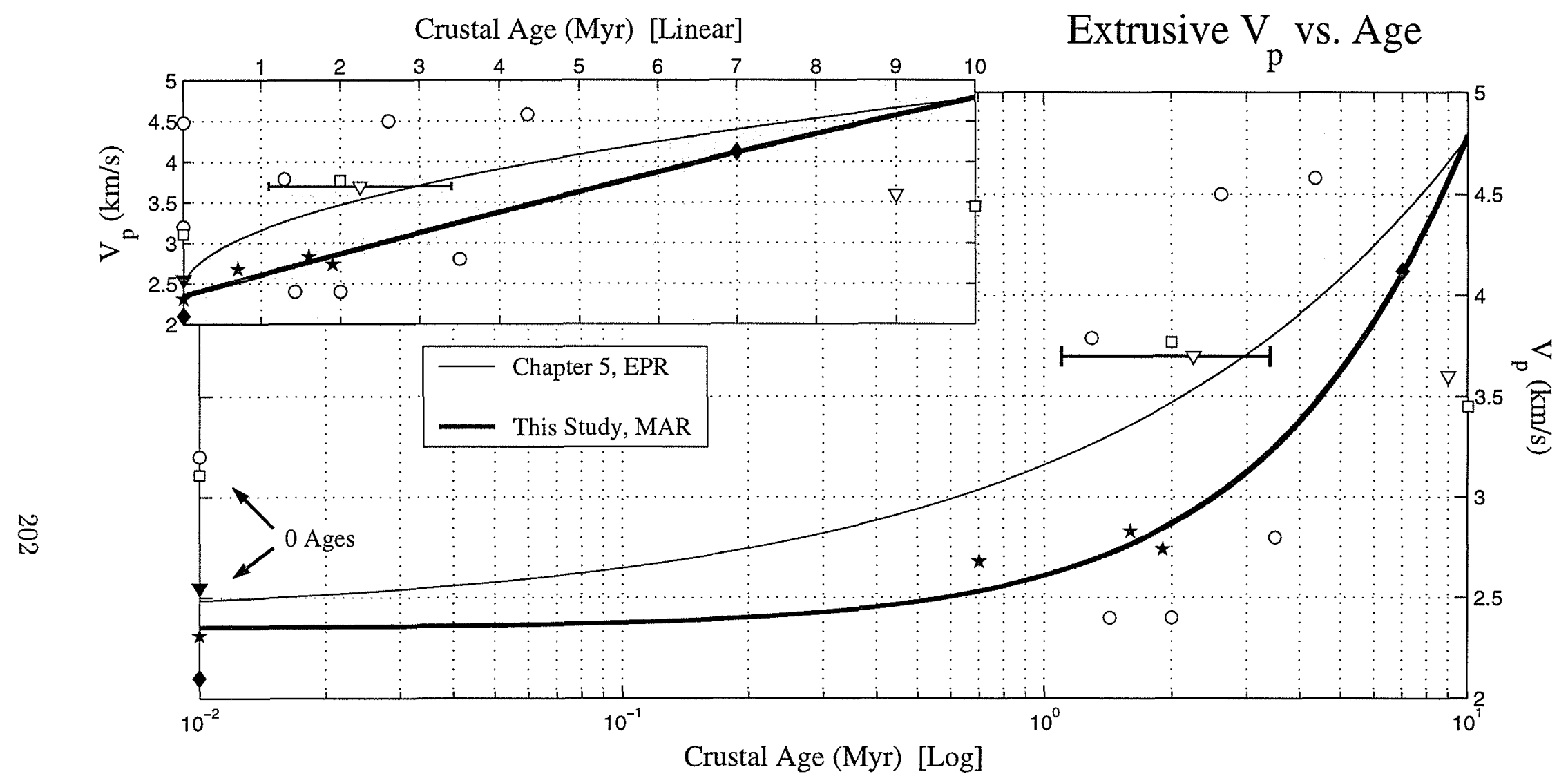

Figure 6-18: P-wave velocity in the extrusive section as a function of crustal age. Refraction results are separated by decade: 1970-1979 (o) [Keen and Tramontini, 1970; Francis and Porter, 1973; Rowlett et al., 1974; Whitmarsh, 1975, 1978], 1980-1989 $(\nabla)$ [White and Purdy, 1983; White and Whitmarsh, 1984; Purdy and Detrick, 1986]. Also shown are results from sonobuoys ( $\square$ ) [Poehls, 1974; Barrett and Purdy, 1979], the on-bottom work of Purdy [1987] ( ), and this study ( $\star$ ). Few determinations of upper crustal velocity have been made at the MAR, particularly in the last decade (filled symbols). Only these recent results were used in the least squares inversion. The best fitting functions for the MAR (thick curve) and EPR (thin curve) [Ch. 5] are shown. The inset plots the data of the larger, log-scale graph as a linear function of age. 
although the presence of a well-established surficial layer shows that it is certainly narrower than the inner valley floor $(<5 \mathrm{~km})$. Outside of the rift valley, particularly along line L, we image a sharper layer $2 \mathrm{~A}$ event (Fig. 6-15) - the result of a thinner off-axis transition zone (Table 6.2). In order to treat the off-axis velocity transition as purely the result of volcanic emplacement, one must establish that post-depositional processes have not modified the velocity profile. This proves to be a difficult task, especially since hydrothermal processes have been linked to a well-established increase in extrusive velocities with age. It is also geologically unreasonable to relate the extremely thin transition zones at sites L1, L2 and L4 to a dike emplacement zone only a few tens of meters wide, since direct observations of the transition zone consistently measure thicknesses of $100 \mathrm{~m}$ or greater (see Chapter 5). Instead, we propose that hydrothermal processes may contribute to a thinning of the transition layer through the preferential deposition of alteration products at the base of the extrusives. The gradual interfingering of high-porosity volcanics and low-porosity dikes within the gradient zone may produce fluid channels that become increasingly impermeable with depth as the extrusive fraction decreases. By focusing hydrothermal fluids through a smaller volume of porous material, the transition zone may provide a more reactive environment in which porosity, and hence velocity, is rapidly altered. The presence of a metamorphic and permeability boundary, stockwork-like sulfide mineralization and significantly altered and demagnetized material in the pillow/dike transition at Hole 504B have led several authors to postulate that this interval was the locus of intense alteration near the ridge axis, where upwelling along fractures in the dikes mixed with cooler seawater circulating in the more permeable extrusive section [Alt et al., 1986; Smith and Banerjee, 1986; Becker et al., 1989; Pariso and Johnson, 1989]. Downhole geophysical logs record a sharper transition zone (50-100 m thick) than suggested by the lithology [Becker et al., 1988]. Similar effects are seen in the lower extrusive sequence of the Troodos, Cyprus and Bay of Islands ophiolites [Moores, 1982; Swift and Johnson, 1984; Johnson and Pariso, 1987]. In light of this argument, 
it seems reasonable to suggest that the thicker transition zones at sites L3 and L5 are a more accurate record of the depositional process, possibly because of less active hydrothermal circulation, especially near the segment end.

What lithology is responsible for the high velocities observed in the shallow crust along line H? Two-ship refraction experiments at the East Pacific Rise show that layer $2 \mathrm{~B}$ velocities typically increase from $5.0-5.8 \mathrm{~km} / \mathrm{s}$ at shallow depths to $5.6-6.6$ $\mathrm{km} / \mathrm{s}$ deeper in the section [Vera et al., 1990; Harding et al., 1989; Kappus et al., 1995; Tolstoy et al., 1997]. The velocities we observe at the base of the transition zone along lines G, I and L correspond well with these shallow dike velocities. The dike section has also been shown to evolve off-axis. At $14^{\circ} \mathrm{S}$ on the EPR, on-axis velocities as low as $5.6 \mathrm{~km} / \mathrm{s}$ are recorded for the impermeable lower dikes immediately above the melt sill [Hussenoeder et al., 1996a; Tolstoy et al., 1997], whereas in 6 Myr-old crust at Hole 504B, velocities of $6.5 \mathrm{~km} / \mathrm{s}$ are reached near the center of the drilled sheeted dike section [Detrick et al., 1994]. Seismic layer 3, which corresponds to the gabbros of the lower crust, is characterized by velocities of $6.4-7.6 \mathrm{~km} / \mathrm{s}$ and a shallower velocity gradient [Raitt, 1963; Shor Jr. et al., 1970; Christenson, and Salisbury, 1975; Houtz and Ewing, 1976; White et al., 1992]. With the velocity overlap between these lithologies, it is difficult to determine whether the high-velocity material at sites $\mathrm{H} 1$ and $\mathrm{H} 2$ represents massive sheeted dikes and sills, or low-velocity, possibly tectonized gabbros. However, given the structural variability along line $\mathrm{H}$, especially near the northern fault scarp and inside corner, we believe the latter to be more reasonable.

The heterogeneous structure along eastern terrace line H (Fig. 6-15b) certainly chronicles a constructional history quite different from the systematic deposition and maturation of extrusives expressed by lines G, I and L. Yet, its surface morphology gives little indication that it is anomalous [D. Smith, personal communication], and submersible dives, performed solely at the flanks of the seamount chain, record (not surprisingly) only volcanics. In contrast to these findings, recent tomographic images of this region reveal a shallow corridor of high velocities beneath the eastern terrace 


\section{[L. Magde, in prep.].}

It is extremely difficult to determine whether the upper crustal variability of the eastern terrace is volcanic or tectonic in origin. A thinner layer 2 may have formed while this section of crust was within the rift valley, if the ridge at that time was undergoing a period of volcanic quiescence. However, we would still expect a complete, albeit thinner, upper crustal sequence from extrusives to dikes to gabbros. Such a well-behaved structure is not recorded at sites $\mathrm{H} 1$ and $\mathrm{H} 2$ (Fig. 6-8), and the two, 700-m-thick units at site $\mathrm{H} 3$ exhibit velocities intermediate to those typical of the volcanic and dike sections. Alternatively, gabbroic material may be located at shallow depths within the crustal section by the mechanical removal of the volcanic carapace. Exposure of the lower crust by large-scale detachment faulting has been documented at several locations along the MAR, especially at inside corner highs [Tucholke et al., 1998]. The high-amplitude reflector dipping toward the active transform (Fig. 6-15b) may be a associated with the tectonic upheaval of inside corner crust rather than a lithologic boundary determined at the time of emplacement. Mutter and Karson [1992] record numerous dipping reflectors in mature slow spreading crust and associate them with remanent detachment surfaces that were successively abandoned as crust spread away from the volcanic center. Another possibility is that this dipping horizon delineates a serpentinization front within the gabbroic section-a consequence of the increased exposure of lower crust and mantle to seawater [Cannat, 1993].

Regardless of geological interpretation, the upper crustal variability observed along line $\mathrm{H}$ and across the rift valley significantly affects the location and analysis of deeper structures. For example, the two-way travel time difference in the upper 500 meters between sites G2 and H3 is nearly $70 \mathrm{~ms}$. Without an accurate estimate of upper crustal velocities, the effective resolution at deeper levels is severely compromised, be it a depth estimate of a lower crustal horizon or a tomographic velocity solution. We must stress, as does Harding et al. [1993], that an accurate model of the upper crust is essential to the interpretation of deeper structure, particularly in a highly variable 
magmatic and tectonic setting such as the MAR.

\subsection{Conclusions}

This study represents the first detailed seismic analysis of extrusive crust at a slow spreading ridge in nearly a decade [Purdy, 1987]. Along with Chapter 5, it is also the first dedicated application of multichannel data to that subject. The velocity functions and seismic images we have generated provide valuable new information on the formation of the shallow crust at slow spreading ridges:

1. At least half, if not all, of the extrusive section is built within the inner valley floor. Off-axis seamounts locally thicken the volcanic pile, but can not account for average extrusive thickness variations with age. We propose that total extrusive thickness is governed instead by residence time and volcanic activity within the inner valley, which is in turn controlled by the episodicity of tectonic vs. magmatic extension (see Hussenoeder et al. [1996b]). Final off-axis layer 2A thickness (extrusive layer plus transition zone) typically ranges from 350-600 m, with moderate along-isochron variability $( \pm 100 \mathrm{~m})$.

2. Three seismic profiles continuously image a layer $2 \mathrm{~A}$ horizon (the first to be observed at a slow spreading center) that is associated with a sharp velocity increase. Its regular appearance indicates that large sections of the upper crust are built by systematic volcanic deposition and subsequently rafted out of the rift valley relatively intact. The highly variable structure and shallow presence of gabbroic (or lower dike) material along the eastern terrace of $\mathrm{OH}-1$ suggest that the regular pattern of crustal construction can be disrupted by periods of volcanic quiescence and/or tectonic modification of the upper crust, especially near segment ends such as the inside corner.

3. Layer $2 \mathrm{~A}$ is observed to thicken toward the Oceanographer fracture zone. This trend may be the result of significant along-axis magma transport, and has important 
implications on the along-axis character of magnetic anomalies at the MAR.

4. The transition zone is significantly thinner in the rift mountains $(<50 \mathrm{~m})$ than within the rift valley $(200-400 \mathrm{~m})$. Thinning of the "seismic" transition may be achieved by enhanced hydrothermal mineralization (i.e., porosity reduction) in the zone of mixed dikes and extrusives.

5. It is hypothesized that porosity reduction due to hydrothermal alteration is responsible for the observed increase in extrusive velocities with age from $2.3 \mathrm{~km} / \mathrm{s}$ on-axis to over $2.7 \mathrm{~km} / \mathrm{s}$ within the young rift mountains.

A comparison of these results to those from other spreading ridges, particularly the fast spreading East Pacific Rise [Ch. 5], significantly increases our limited understanding about how spreading rate affects the construction and modification of upper oceanic crust. The major similarities and differences between our study area and faster spreading ocean ridges (EPR \& JDFR) are as follows:

1. The thick layer of pure extrusives in the rift valley is similar to on-axis estimates for the intermediate spreading JDFR, but is at least twice as thick as at the rise crest of the EPR. This indicates that, although a similar total volume of extrusives is produced per kilometer of spreading at all mid-ocean ridges, the pattern of extrusive thickening at the fast spreading EPR may differ from that at the slower spreading MAR and JDFR.

2. The similar transition zone thickness between the MAR, JDFR and EPR (200$400 \mathrm{~m}$ ) suggests that the zone of dike emplacement may be focused at all spreading: ridges, and that processes affecting the deposition of lavas (i.e., fault damming, eruptive volume) may be responsible for the on-axis thickness differences observed.

3. In general, basement velocities increase more rapidly in fast spreading crust than in slow spreading crust. This may be a consequence of enhanced hydrothermal activity (i.e., porosity reduction) needed to cool the more volcanically active fast spreading centers. 


\section{Chapter 7}

\section{Conclusions}

\subsection{Synthesis of Results}

This thesis describes the shallow magnetic and seismic structure of two contrasting mid-ocean ridges: the fast spreading East Pacific Rise and the slow spreading MidAtlantic Ridge. Our analysis was facilitated by the inversion techniques developed in this thesis. The direct inversion method of Chapter 2 allows us to maximize the sensitivity of near-bottom magnetics to the fine-scale variability of the magnetic source layer (layer 2A). With its ability to efficiently extract velocities from multichannel seismic data, the genetic algorithm (Appendix A) provides additional information about the internal structure of the upper crust. The seismic and magnetic constraints presented in this thesis have helped us to better understand the roles of volcanic, tectonic and hydrothermal processes at fast and slow spreading ridges.

The continuous AMC reflector observed along large portions of the East Pacific Rise is the subject of a detailed amplitude and waveform analysis in Chapter 4 . The magma body thought to be responsible for this event is widely regarded as the primary source of melt erupted at the seafloor, and is therefore a key factor in the formation of the upper crust. At five sites along the EPR, we find that the magma sill is thin $(<100$ m) and crystal-rich. These properties may inhibit lateral magma transport along the 
rise crest and restrict the volume of melt available for eruption. The variability of magma sill properties and their correlation with known and inferred eruptive events indicate that the magma sill is not a reflection of the long-term magma budget to the rise crest, but is rather a direct measure of the current volcanic state of the rise axis within its 10 - to 100-year eruption cycle.

In Chapter 5, we examine the shallow seismic stucture of the EPR at $17^{\circ} 20^{\prime} \mathrm{S}$ and find a double-step velocity structure at the rise crest. We propose that the two velocity contrasts, one at a basement depth of 70-100 $\mathrm{m}$ and the other at $250-350 \mathrm{~m}$ depth, represent the top and bottom of the pillow/dike transition, respectively, The shallower of the two is observed to deepen by $300-400 \mathrm{~m}$ within $1-4 \mathrm{~km}$ of the rise axis - a thickening profile that suggests volcanic emplacement is highly focused at the EPR. For this reason, we label the shallower horizon as the base of seismic layer 2A. The deeper of the two horizons is not observed off-axis. Hydrothermal alteration may be enhanced at the base of the transition zone by the difference in rheology and deformation styles between extrusives and dikes, thus diminishing the initial velocity contrast beyond the resolution of our seismic methods.

By combining our seismic and magnetic results of Chapters 3 and 6 , we can construct a more complete picture of the geological processes at the Mid-Atlantic Ridge than we could with either method alone. In Chapter 3, we learn that the central anomaly magnetization high (CAMH) is sharply peaked and marks the location of focused volcanic accretion. This result is supported by the thick layer of pure extrusives observed along the inner valley floor of segment $\mathrm{OH}-1$ (Ch. 6), which indicates that the zone of dike emplacement is quite narrow compared to the lateral extent of lava flows. The clear seismic images of the pillow/dike transition along the elevated terraces of OH-1 (Ch. 6) and the off-axis blocks of unaltered crust north of the Kane transform (Ch. 3) suggest that the well-developed volcanic stratigraphy generated on-axis is often preserved outside the rift valley. These unaltered crustal sections may be episodically transported into the rift mountains by zones of intense faulting 
and hydrothermal alteration. We propose that post-emplacement processes are the dominant mechanisms for generating cross-axis variability in the upper crust at slow spreading ridges, not volcanic construction. Along-axis transport of lava through lateral dike injection may thicken the extrusive section away from segment centers. A thicker, more $\mathrm{Fe}$ - and Ti-rich extrusive layer may be responsible for the stronger CAMHs toward the segment ends of slow spreading ridges.

Since the seismic methods used in the last two chapters are nearly identical, the upper crustal structure of a fast and slow spreading ridge can be directly compared for the first time. A surprising result of this comparison is that the extrusive section achieves a final thickness of 300-500 m regardless of spreading rate. The exact pattern of thickening within the inner valley floor of the MAR remains unknown. However, the similar transition zone thicknesses observed at both ridges suggest that volcanic emplacement at the the MAR may be quite focused, as it is at the EPR. Both spreading ridges exhibit a rapid off-axis modification of the pillow/dike transition. We propose that this zone of mixed lithologies is the site of enhanced hydrothermal mineralization. Basement velocities increase more rapidly with age at the EPR than at the MAR. This may be an indication of less vigorous hydrothermal circulation at slow spreading ridges. A similar, perhaps coincidental, difference exists in the observed magnetization decay with age, which is also associated with alteration processes (albeit lower temperature). These differences, however, may be a consequence of the greater disparity between apparent and true age at slow spreading ridges.

\subsection{Future Work}

In many respects, the progress of observation-based sciences is dictated by the ability of acquisition and analysis methods to provide the information necessary to address key questions. For this reason, advances in technology and data processing are crucial to a more complete understanding of mid-ocean ridges and upper oceanic crust. The 
direct inversion in Chapter 2 and the genetic algorithm of Appendix A are two such analysis methods that have provided more detailed information than previously available. Their broad applicability also allows them to be extended to other acquisition methods. The Canadian Geological Survey is currently adapting the direct inversion for use with 3-D data sets [M. Pilkington, personal communiation, 1998]. Plans also exist to apply the GA waveform inversion to an on-bottom seismic data set recently acquired at the Mid-Atlantic Ridge [J. Collins, personal communication, 1998]. Because of its high lateral and depth resolution, the combination of on-bottom seismics and the GA is an excellent approach to unresolved questions regarding the shallow crustal structure in bathymetrically extreme areas such as the MAR. For example, what is the shape of the extrusive thickening profile across the inner valley floor?

The recent installation of a 6-km streamer aboard the R/V Maurice Ewing greatly increases the amount of information recorded by multichannel seismics. A 6-km streamer will not only record refractions from deep within the dike section (and possibly the gabbros, depending on basement depth), it will allow us to examine the shallow velocity structure of areas in excess of $3 \mathrm{~km}$ in depth. This includes the distal flanks of the EPR and the inner valley along most of the MAR. In the absence of a deep-tow streamer (or on-bootom cable), on-bottom seismics and 6-km-aperture MCS are the next best thing!

The recent Conference on the Magnetization of Oceanic Crust [Johnson et al., 1997] recognized the importance of combining magnetic and seismic techniques to answer key questions about the accretion and evolution of the upper crust, and to test whether the magnetic source layer, seismic layer $2 \mathrm{~A}$ and the extrusives section are one and the same. Attempts to link the upper crustal magnetic signal to layer 2A thickness have been made for the Juan de Fuca Ridge [Tivey and Johnson, 1993; Tivey, 1994] and EPR [Gee and Kent, 1994]. Unfortunately, these studies either used a simplified crustal model instead of seismically determined thicknesses [Gee and Kent, 1994] or compare magnetic results to spatially averaged marine refraction 
data [Tivey and Johnson, 1993; Tivey, 1994]. Only recently, through the analysis of coincident multichannel seismic and near-bottom magnetic lines, have we begun to convincingly establish such a correlation [Schouten et al., 1998]. By using both techniques together to mutually establish baselines for comparison, we can learn considerably more about the properties of the upper crust than we can with either method alone. Ultimately, the goal of this approach is to formulate an integrated geophysical model for the formation and evolution of young upper oceanic crust that is valid at all spreading rates. 


\section{Appendix A}

\section{The Genetic Algorithm}

The genetic algorithm is an iterative nonlinear optimization/search method based on concepts of biological natural selection. Since its inception over 20 years ago [Holland, 1975], the GA has been applied to a variety of problems, which only recently have begun to include the inversion of seismic data [Stoffa and Sen, 1991; Sambridge and Drijkoningen, 1992; Sen and Stoffa, 1992; Nolte and Frazer, 1994; Drijkoningen and White, 1995; Mallick, 1995; Boschetti et al., 1996; Harding and Kappus, 1996; Tolstoy et al., 1997]. Its applicability to a broad spectrum of problems is rooted in its ability to simultaneously examine a population of models through an objective fitness function (Fig. A-1). By using components of better fitting models as building blocks for new ones, it robustly searches model space for a global optimum. The GA is composed of three basic operations: reproduction, crossover, and mutation (Fig. A-1). Initially, several models, $M$, are generated in a quasi-random fashion and discretized into a unique coding. The reproduction step evaluates the fitness of each model and selects pairs of models in proportion to their fitness estimates. Crossover swaps coded pieces of these models at random locations, and mutation changes the value of each coding cell (i.e., gene or bit) with a specific probability. For a thorough treatment on the nature of GAs, see Goldberg [1989], Davis [1991], and Sen and Stoffa [1995].

Although the mechanics of a GA is quite simple, its application to specific prob- 
M Velocity-Depth Models

M Bitstrings
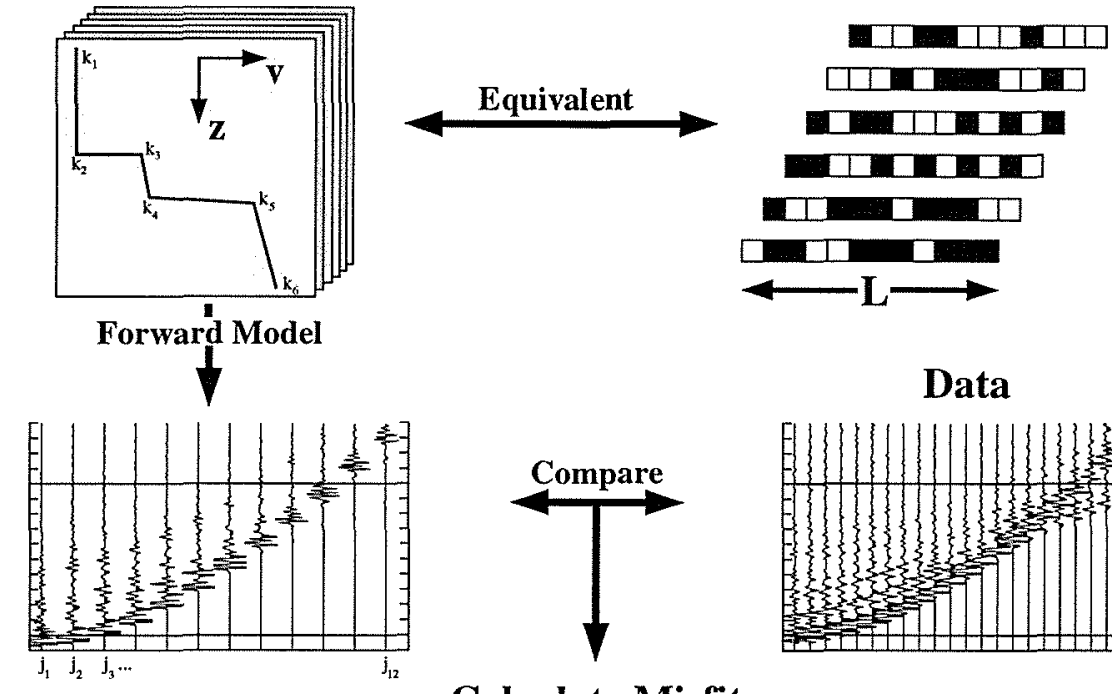

Data
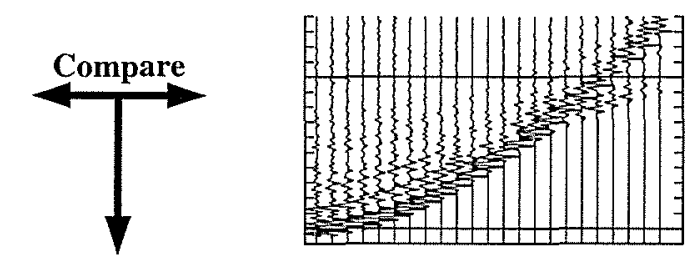

Calculate Misfit

$\phi=\phi_{w f}+\mu \phi_{t t}$

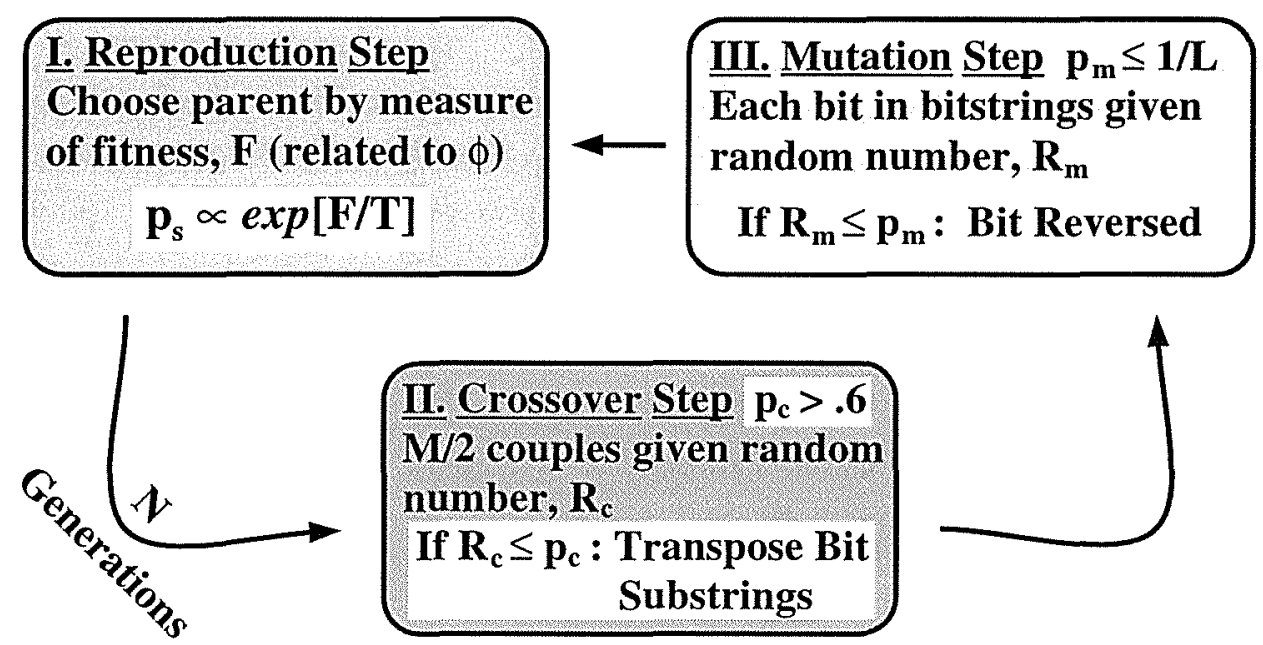

Figure A-1: Flow diagram for applying the genetic algorithm to seismic waveform inversion. A population of velocity-depth models, each with its own unique binary coding, is generated at every iteration. Within an iteration, an objective misfit, $\phi$, between each model and the true structure is calculated by comparing its corresponding synthetic seismograms to the data. This includes both waveform, $\phi_{w f}$, and travel time, $\phi_{t t}$, information. Models then undergo the three-step process of reproduction, crossover and mutation, which generates a new model population to compare to with data. We assign $j$ to the traces participating in the misfit calculation, $k$ to the velocity model nodes, $L$ to the number of bits in the coded strings ( $2 K$ in our case), $M$ to the number of models per generation, and $N$ to the number of generations in a run. 
lems, such as seismic waveform inversion require a more rigorous definition. The best model coding scheme, fitness function, and probabilistic rules that govern its three basic steps are specific to each application. These processes must be optimized to provide the GA with the best possible means of efficiently reaching a robust solution.

\section{A.1 Model Parameterization}

The initial population of velocity-depth functions is generated at random from a fixed set of velocity nodes, $\mathbf{v}$. The depth to the $k^{\text {th }}$ velocity node in each model is given by,

$$
z_{k}=z_{o}+\sum_{i=1}^{k} \delta z_{i} \cdot r_{i} \quad 0 \leq r_{i} \leq 2 b-1
$$

where $z_{o}$ is the water depth, and every depth increment is the product of the depth scaling, $\delta z$, and a randomly generated number, $r$. Thus, for a predefined set of velocities and depth scalings, a unique coding for each model is contained in the decimal coding vector, $\mathbf{r}$. The maximum value of components in $\mathbf{r}$ is governed by the number of bits, $b$, allowed to characterize each node in binary form. By requiring velocity nodes to increase with depth, this coding scheme precludes the existence of velocity inversions, but not of positive velocity contrast interfaces.

The mean starting model generated by this parameterization can be written as,

$$
\bar{z}_{k}=z_{o}+\sum_{i=1}^{k} \delta z_{i} \cdot\left(b-\frac{1}{2}\right)
$$

and is treated as the reference model around which the initial population is randomly generated. The summation forces each node to be cumulatively dependent on all overlying nodes. This ensures that the initial population is distributed in a Gaussian fashion about the reference model, with larger standard deviations at depth. A reference model may be created from a predefined velocity-depth function by resampling its velocities, $\mathbf{v}$, at the mean depth values, $\overline{\mathbf{z}}$. The values used to parameterize the 
models of Chapter 5 are given in the top portion of Table A.1.

\section{A.2 Fitness Calculation}

To make the GA equally sensitive to both layer $2 \mathrm{~A}$ and $2 \mathrm{~B}$ events, the weaker $2 \mathrm{~B}$ traces are weighted by the ratio of the two trace envelopes (modulus of the complex trace) [Cary and Chapman, 1988]. This weighting is calculated for each individual supergather, since the $2 \mathrm{~A} / 2 \mathrm{~B}$ ratio tends to vary spatially. Model synthetics are similarly scaled to the data seismograms on a window-by-window basis, which allows the synthetics to match the amplitude pattern within an individual refraction event. At each iteration, fitnesses of the velocity models in the current population are calculated by comparing their corresponding synthetic seismograms with the data only within the two (scaled) windows enclosing the layer $2 \mathrm{~A}$ and $2 \mathrm{~B}$ events.

Two basic steps comprise the fitness calculation: misfit computation and fitness scaling. The misfit of each model is calculated with respect to the data, and consists of a travel time, $\phi_{t t}$, and waveform, $\phi_{w f}$, component:

$$
\phi=\phi_{w f}+\mu \phi_{t t}
$$

We can represent the misfit of a single trace, $j$, as,

$$
\phi_{j}=P \bar{D}^{-1} \sqrt{D_{j}^{2}+S_{j}^{2}-2 \rho\left(t_{j}\right)}+\mu\left|t_{j}\right|
$$

where $P$ is the dominant period of the windowed event, $\bar{D}$ is the root-mean-square amplitude of both events in the data, $D^{2}$ and $S^{2}$ are the mean squared amplitudes of the data and corresponding synthetic trace, respectively, and $\mu$ is a scaling factor (Table A.1). The travel time misfit, $\phi_{t t}$, is simply the magnitude of $t$, the time lag closest to zero that produces a local maximum in the non-normalized cross-correlation $(\rho)$ and hence a minimum in waveform misfit $\left(\phi_{w f}\right)$. The total travel time and waveform 
Table A.1: Tested and Applied ${ }^{a}$ Parameter Settings for the Genetic Algorithm

\begin{tabular}{|c|c|c|c|c|}
\hline Parameter & Test Runs & On-Axis & 3-km Off & 6-km Off \\
\hline Bits per Node, $b$ & 2 & 2 & 2 & 2 \\
\hline Depth Scaling, & $10<4.0$ & $3.3<4.5$ & & \\
\hline$\delta z$ (meters) & $20<5.0$ & $10<5.0$ & $7<5.1$ & $8<5.2$ \\
\hline at velocity $(\mathrm{km} / \mathrm{s})$ & $40>5.0$ & $30>5.0$ & $21>5.1$ & $24>5.2$ \\
\hline Max. Vel. Modeled & $5.6 \mathrm{~m}$ & $5.4 \mathrm{~m}$ & $5.6 \mathrm{~m}$ & $5.8 \mathrm{~m}$ \\
\hline Max. Depth Modeled & $\sim 1.5 \mathrm{~km}$ & $1 \mathrm{~km}$ & $1 \mathrm{~km}$ & $1 \mathrm{~km}$ \\
\hline Nodes per Model, $K$ & 43 & $37-55$ & $40-72$ & $33-66$ \\
\hline Generations per Run ${ }^{c}, N$ & $80,40^{d}, 20$ & $40\left(\right.$ or $\left.14^{b}\right)$ & $40\left(\right.$ or $\left.14^{b}\right)$ & $40\left(\right.$ or $\left.14^{b}\right)$ \\
\hline Models per Gen. ${ }^{c}, M$ & $50,100^{d}, 200$ & $100\left(\right.$ or $\left.500^{b}\right)$ & $100\left(\right.$ or $\left.500^{b}\right)$ & $100\left(\right.$ or $\left.500^{b}\right)$ \\
\hline Mutation Prob. ${ }^{c}, p_{m}$ & $0.003,0.03^{d}, 0.01$ & 0.008 & 0.008 & 0.008 \\
\hline Crossover Prob. $^{c}, p_{c}$ & $0.6,0.8^{d}, 0.95$ & 0.8 & 0.8 & 0.8 \\
\hline Update Prob. ${ }^{c}, p_{u}$ & $0.0^{d}, 0.3,0.6,0.9$ & 0.0 & 0.0 & 0.0 \\
\hline Dominant Period, $P$ & $\frac{1}{15} \mathrm{sec}$ & $\frac{1}{15} \mathrm{sec}$ & $\frac{1}{15} \mathrm{sec}$ & $\frac{1}{15} \mathrm{sec}$ \\
\hline Weighting Factor, $\mu$ & $8^{-2}$ & $8^{-2}$ & $8^{-2}$ & $8^{-2}$ \\
\hline Sigma Truncation $^{c}, c$ & $1,2^{d}, 3$ & 2 & 2 & 2 \\
\hline Max-Mean Ratio $^{c}, R$ & $1.2,1.6^{d}, 2.0$ & 1.6 & 1.6 & 1.6 \\
\hline
\end{tabular}

${ }^{a}$ Applied to the East Pacific Rise data set of Chapter 5

${ }^{b}$ Initial seafloor search run (first of the cascaded runs)

${ }^{c}$ Parameter was optimized by test runs

${ }^{d}$ Best setting during test runs 
misfit for the model seismograms is taken as the root-mean-square of the individual trace misfits. In the limiting case where the data seismograms are equivalent to the synthetics, the waveform and travel time misfits are zero.

We use a conversion from misfit to raw fitness that employs variance information to remove exceptionally bad models from the population [Forrest, 1985; Goldberg, 1989]. The relationship between the raw fitness and misfit of a model, $m$, can be regarded as,

$$
f_{m}=(\bar{\phi}+c \sigma)-\phi_{m}
$$

where $\bar{\phi}$ is the average population misfit, $f$ is the raw fitness, $\sigma$ is the standard deviation of the population misfit, and $c$ is a constant called the sigma truncation factor. All models with a misfit greater than $c \sigma$ above the mean will have a negative fitness. By setting the fitness of such models to zero, we effectively prevent them from propagating their genetic information to future generations. Zeroing negative fitnesses ensures that no negative fitness values are generated during the scaling process, regardless of the method used.

Scaling of the raw fitness vector ensures that moderate levels of competition are maintained throughout the entire GA run (i.e., convergence is kept at an appropriate rate). Because the population variance is greatest early on, subduing the wide distribution of fitnesses can prevent a small number of good models from dominating the selection process. Later, as the population becomes more homogeneous, fitness scaling can accentuate differences between models, thereby maintaining convergence.

Although linear scaling of the fitness function is simple, it is often not powerful enough near the end of a simulation, when the population is nearly homogeneous. We therefore use an exponential scaling of the raw fitness function [Sen and Stoffa, 1992, 1995; Stoffa and Sen, 1991],

$$
\mathbf{F}=\exp \left(\frac{\mathbf{f}}{T}\right)
$$


where the scalar $T$ is a stretching factor, or temperature. The use of temperature in the fitness scaling is a concept borrowed from simulated annealing, an optimization technique that uses random processes to search for minimum energy states in model space. As $T \longrightarrow 0$, the raw fitness function is stretched, or in other words:

$$
1 \leq \frac{\max (\mathbf{F})}{\operatorname{mean}(\mathbf{F})}=R \leq \infty
$$

The max-mean ratio, $R$, is the number of times the best population member is expected to be chosen in the selection process. If $R$ is kept constant throughout a run, then the corresponding temperature, $T$, at each generation is a proxy for population variance, or level of convergence. The selection probability, $p_{s}$, of each model, $m$, is then the ratio of its scaled fitness to the sum of the all scaled model fitnesses in that population,

$$
p_{s, m}=\frac{F_{m}}{\sum F_{m}}=\frac{\exp \left(\frac{f_{m}}{T}\right)}{\sum \exp \left(\frac{f_{m}}{T}\right)}
$$

such that,

$$
\sum p_{s}=M
$$

The summation is over all models in that population, $M$. The selection probability vector is then scaled by its mean so that it corresponds to the number of times each model is expected to be chosen in the selection process.

Models are chosen by stochastic remainder selection without replacement. This method selects models in direct proportion to the integer part of their selection probability, $p_{s}$. The fractional parts are then used as success probabilities in a random selection process that is continued until the selected population is full. Unlike the integer selection portion, models that have their remainders chosen are not returned to the pool of available candidates. 


\section{A.3 Convergence}

The GA is an intelligent search algorithm that is directed by information it gathers about the model space at each iteration. Its convergence rate is significantly faster than that of enumerative schemes, such as Monte Carlo and grid search methods. However, a poor choice of parameter settings (discussed above) can severely degrade convergence. We determined the optimal setting for each of these parameters by testing a range of values on a synthetic data set, generated by reflectivity modeling a predefined velocity function. Our reference starting model was placed farther than one standard deviation from the true velocity model. The average convergence efficiency after 4000 trial evaluations was calculated for each parameter setting and compared to a control run in which none were changed. Ten separate runs were averaged for each comparison. Figure A-2 shows the average convergence characteristics of the optimal parameter settings. Table A.1 presents these test results and the values used in our analysis of the East Pacific Rise data set [Ch. 5].

The parameters that most affect convergence and the optimal value of other parameters are those that govern the coding scheme, namely the number of bits per node, $b$, and the depth scaling, $\delta z$. In our formulation, two bits per node provide sufficient variability (i.e., four depth spacings) to sample a significant part of model space, while still generating a coded bitstring of manageable size. A depth scaling that increases with velocity (i.e., with depth [Table A.1]) retains high resolution in the upper crust, and is consistent with a decrease in vertical resolution with depth. The chosen depth scaling is generally smaller than that given by the canonical one-quarter wavelength resolution criteria for two reasons. One is that we use amplitude information, which aids in the resolution of features below this threshold. Secondly, for our coding scheme to generate a sufficient number of nodes along the thin transition zone, a small depth scaling is required. Given a maximum depth extent of velocity models, $\delta z$ and $b$ determine the number of nodes per model, $K$, and their product gives the size of the binary model space, $L$. 

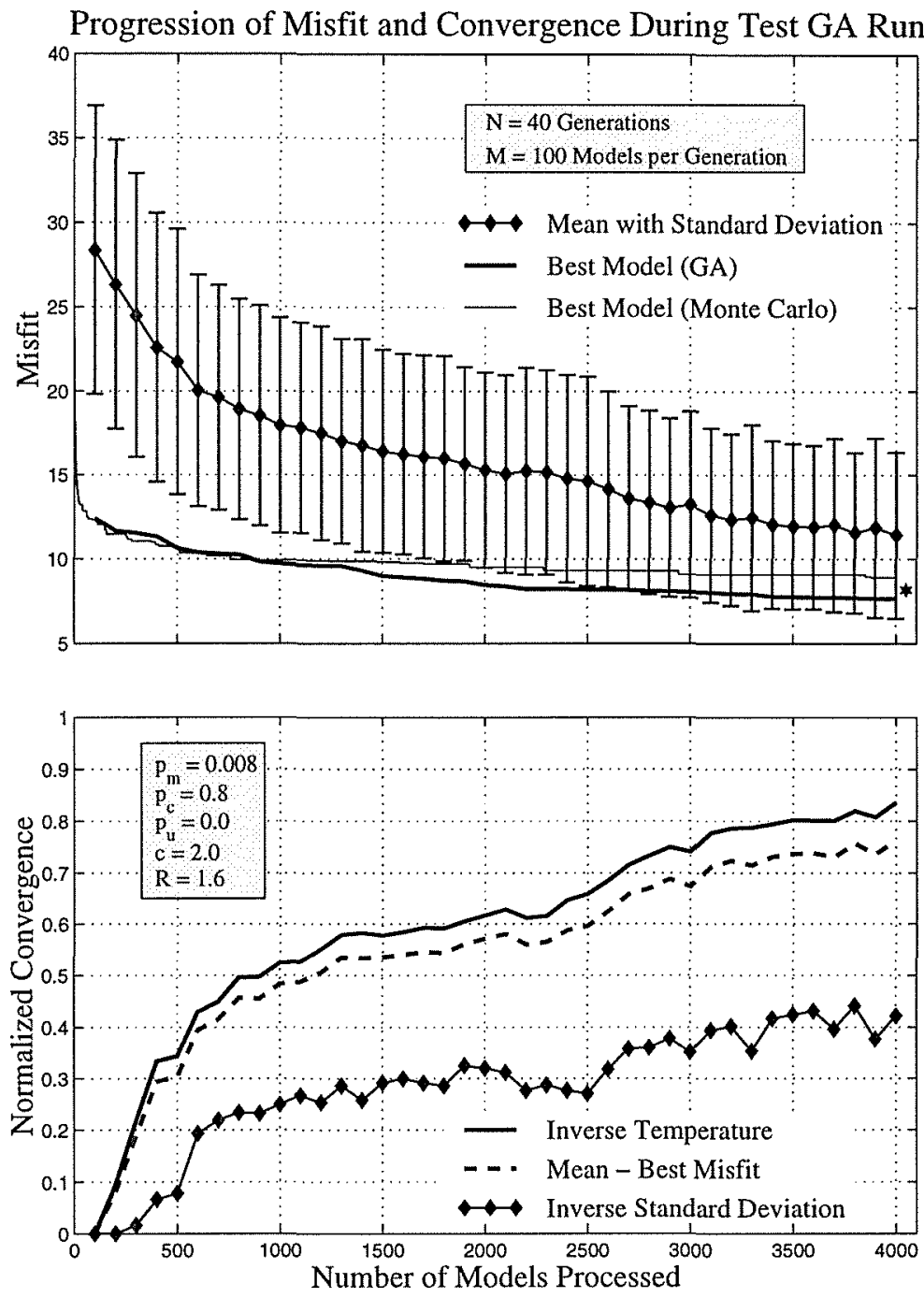

Figure A-2: Progression of a test GA run performed on a synthetic data set. The average of 10 simulations is shown. Parameter settings specific to this run are shown in grey panels. All other settings are in Table A.1. (top) Mean population misfit (diamonds) and best model misfit (thick line) at each generation. As the algorithm progresses and misfit decreases, the mean misfit approaches that of the best model and the population becomes more homogeneous (i.e., variance decreases). This is compared to the average convergence of 10 Monte Carlo, or random, searches (thin line). The best misfit after randomly sampling 40,000 models (star) is still larger than that of the GA after sampling one-tenth as many models. (bottom) The population variance (diamonds) and offset between the mean and minimum misfit (dashed) are measures of the asymptotic convergence. At the end of the run, the mean misfit is $80 \%$ closer to that of the best model, even though the misfit of the best model is significantly lower than at the outset. The decrease in population variance is less pronounced because of a non-zero mutation rate. The stretching factor (or temperature, $T$ ) of Equation A.6 is also a measure of convergence (solid), since it is constrained by the ratio of best model to mean model fitness (Eq. A.7). 
Model parameterization also influences the optimal population size, $M$, number of generations per run, $N$, and mutation probability, $p_{m}$. Population size can not be so small that it introduces too little genetic variability into the gene pool, but it can not be so large that its inertia degrades the population's ability to change as a group. Convergence occurs when the statistics of a population no longer change from one generation to the next. For the optimal population size of 100, this occurs near 40 generations (Fig. A-2). In order to preserve continuity across generations, mutation rate is generally less than the inverse of the bitstring length, $L$. Although mutation rate guards against the permanent loss of genetic information, high values degrade the directedness of the search and cause performance to resemble that of a random search. Varying crossover, $p_{c}$, and update probability, $p_{u}$, are also means of retaining genetic information from one generation to the next. Crossover probability is the likelihood that the chosen parents swap bit substrings to create new offspring, rather than move into the next generation unaltered. Convergence is generally highest when this value is kept large. Update probability is the likelihood that, if a randomly chosen model from the parent population has a greater fitness than the current child, it will replace that offspring. Although previous authors [Stoffa and Sen, 1991; Sen and Stoffa 1992] show that a high $p_{u}$ increases the performance of certain GA schemes, our implementation is optimized when no parent models are carried forward to the next generation in this fashion (i.e., $p_{u}=0$ ).

It is extremely important that the correct seismic information drive convergence by being appropriately represented in the misfit calculation. Hence, the dominant period, $P$, and weighting factor, $\mu$, were chosen to optimize sensitivity to both travel time and waveform components. As stated earlier, the conversion from misfit to scaled fitness is essential to maintaining intermediate levels of convergence at the beginning and end of a simulation. The misfit cutoff, $c \sigma$, is generally chosen to be a low multiple of the population standard deviation $(1 \leq c \leq 3)$. Smaller values will exclude too much of the population, and larger values are ineffective at driving 
convergence. In order for convergence to occur at all, the fitness function must be scaled such that the max-mean ratio, $R$, is above unity. Performance is generally optimized for $1 \leq R \leq 2$, with larger values driving convergence more strongly. Values above this threshold result in premature convergence.

The final factor affecting convergence is sensitivity to starting model. Although the GA is among the most effective global optimization methods, solutions far outside the region sampled by the initial population are unlikely to be analyzed. This region typically corresponds to twice the standard deviation bounds of the starting population. We minimize this problem by performing several inversions on each data set, using the mean final model of one run as the mean starting model to the next. The practice of cascading several GA runs reduces starting model sensitivity by allowing successive simulations to migrate toward better fitting regions that may be far from the first starting model. Generally, the GA was cascaded three times for each supergather, with the first run tailored to find the seafloor velocity. The population dynamics for the first run were modified, because the coding scheme of Equation A.1 uses a fixed set of velocities, and therefore does not allow velocity to vary at the seafloor. We force the seafloor velocity to be a free variable by introducing into the simulation several distinct subpopulations, each with a different seafloor velocity, that compete but do not mix. The subpopulation with the most members at the end of the run is the most fit class of models, from which the mean starting model for the subsequent inversions is generated. The depth nodes of each mean starting model are appropriately. scaled so that the two-way travel times remain the same. In Chapter 5, we use five subpopulations, whose seafloor velocities are multiples of $0.1 \mathrm{~km} / \mathrm{s}$ different from that at the adjacent location (Table A.1). In this fashion, seafloor velocity differences of up to $0.2 \mathrm{~km} / \mathrm{s}$ between adjacent supergathers are detected by the inversion process. Since no study locations adjoin in Chapter 6, the five subpopulations are centered about a mean starting model that was derived from hand-modeling the travel times. 


\section{A.4 Error Analysis}

The probability that model " $m$ " is the global optimum can be calculated by the posteriori probability density (PPD) function at the end of a GA run:

$$
\Psi_{m}=\frac{\exp \left(\frac{f_{m}^{\prime}}{T}\right)}{\sum \exp \left(\frac{f_{m}^{\prime}}{T}\right)}
$$

This is the Gibbs probability distribution, which is calculated in the same fashion as

the selection probability (Eq. A.8). The raw fitness, $f^{\prime}$, differs from that calculated at the end of each generation (Eq. A.5) in that the statistics of the entire population are used to truncate the misfit. The stretching factor, $T$, remains the same as in Equation A.6, and is the temperature associated with that model's generation. The summation is over all models analyzed during the run $(N \cdot M)$. This formulation assigns higher probabilities to models generated at lower temperatures. Hence, the most significant portion of the PPD function is sampled late in the simulation, when the GA has converged toward the best fitting region of model space.

The mean final model can be calculated by weighting each model of the simulation with its PPD and then summing:

$$
\overline{\mathbf{Z}}=\sum_{i=1}^{N \cdot M} \mathbf{z}_{i} \Psi_{i}
$$

This differs from the mean starting model of Equation A.2 in that the PPD function is not uniform and, since the GA is a directed search algorithm, models are not distributed in a Gaussian fashion after the first generation. In our parameterization, the array of models, $\mathbf{z}$, can be either that of depth increment $(\delta z \cdot r$ in Equation A.1) or of total depth. The two results are identical if we take the additional step of applying Equation A.1 to the final depth increments.

If we treat our depth models as column vectors, the covariance matrix, whose 
diagonal is the variance of the mean final model, is given by,

$$
C O V=\sum_{i=1}^{N \cdot M} \mathbf{z}_{i} \mathbf{z}_{i}^{T} \Psi_{i}-\overline{\mathbf{Z}} \overline{\mathbf{Z}}^{T}
$$

where ${ }^{T}$ is the transpose. Once again, this can be calculated for depth increment or total depth. Since total depth is a function of the depth increment, an independent variable, the variance corresponding to the mean final model of each will differ. From a geological standpoint, we are interested in the total depth variation of each node below the seafloor. We therefore do not compute the variance of the mean final velocity increment model.

Since the GA uses the fitness of prior models to direct its search process, it samples model space unevenly. This uneven distribution of sampled models makes error analysis difficult, because it may bias the PPD function by undersampling certain regions of model space. The practice of cascading several GA runs also minimizes this problem. By using previous information on the model space to center the initial population of the next run around the region that contributes most to the PPD (i.e., region of highest fitness), sampling is more evenly distributed about the global optimum. 


\section{Bibliography}

Agar, A. M., and K. D. Klitgord, A mechanism for decoupling within the oceanic lithosphere revealed in the Troodos ophiolite, Nature, 374, 232-238, 1995.

Agar, S. M., Fracture evolution in the upper ocean crust: Evidence from DSDP Hole 504B, in Deformation Mechanisms, Rheology and Tectonics, edited by R. J. Knipe and E. H. Rutter, Geol. Soc. Spec. Publ. No. 54, pp. 41-50, The Geological Society of America, Boulder, Colorado, 1990.

Aki, K., and P. G. Richards, Quantitative seismology, theory and methods, vol. 1, W.H. Freeman \& Co., 1980.

Alabaster, T., J. A. Pearce, and J. Malpus, The volcanic stratigraphy and petrogenesis of the Oman ophiolite complex, Contrib. Mineral. Petrol., 81, 168-183, 1982.

Allerton, S., B. J. Murton, R. C. Searle, and M. Jones, Extensional faulting and segmentation of the Mid-Atlantic Ridge north of the Kane fracture zone $\left(24^{\circ} 00^{\prime} \mathrm{N}\right.$ to $\left.24^{\circ} 40^{\prime} \mathrm{N}\right)$, Mar. Geophys. Res., 17, 37-61, 1995.

Alt, J. C., J. Honnorez, C. Laverne, and R. Emmermann, Hydrothermal alteration of a $1 \mathrm{~km}$ section through the upper oceanic crust, Deep Sea Drilling Project Hole 504B: Mineralogy, chemistry, and evolution of seawater-basalt interactions, J. Geophys. Res., $91,10,309-10,335,1986$.

Anderson, R. N., M. D. Zoback, S. H. Hickman, and R. L. Newmark, Permeability versus depth in the upper oceanic crust: In situ measurements in DSDP Hole 504B, eastern equatorial Pacific, J. Geophys. Res., 90, 3659-3669, 1985.

Anderson, R. N., et al., DSDP Hole 504B, the first reference section over $1 \mathrm{~km}$ through layer 2 of the oceanic crust, Nature, 300, 589-594, 1982.

Auzende, J.-M., D. Bideau, E. Bonatti, M. Cannat, J. Honnorez, Y. Lagabrielle, J. Malavieille, V. Mamaloukas-Frangoulis, and C. Mevel, Direct observation of a section through slow-spreading oceanic crust, Nature, 337, 726-729, 1989.

Auzende, J.-M., et al., Recent tectonic, magmatic, and hydrothermal activity on the East Pacific Rise between $17^{\circ} \mathrm{S}$ and $19^{\circ} \mathrm{S}$ : Submersible observations, J. Geophys. Res., 101, 17,995-18,010, 1996. 
Ballard, R. D., and T. H. Van Andel, Morphology and tectonics of the inner rift valley at lat $36^{\circ} 50^{\prime} \mathrm{N}$ on the Mid-Atlantic Ridge, Geol. Soc. Am. Bull., 88, 507-530, 1977.

Barclay, A. H., D. R. Toomey, and S. C. Solomon, Seismic structure and crustal magmatism at the Mid-Atlantic Ridge, $35^{\circ} \mathrm{N}$, submitted to J. Geophys. Res., 1997.

Barrett, D. L., and G. M. Purdy, IPOD survey area AT-6: Seismic refraction results, Initial Rep. Deep Sea Drill. Proj., 45, 49-53, 1979.

Barth, G. A., J. C. Mutter, and R. S. Detrick, Along-axis variability on East Pacific Rise seismic structure, Eos Trans. AGU, 68, 1491-1492, 1987.

Becker, K., et al., In situ electrical resistivity and bulk porosity of the ocean crust, Costa Rica Rift, Nature, 300, 594-598, 1982.

Becker, K., et al., in Site 504, Costa Rica Rift, no. 111 in Init. Rep. Ocean Drill. Prog., part A, pp. 35-251, Ocean Drill. Prog., College Station, TX, 1988.

Becker, K., et al., Drilling deep into young oceanic crust, Hole 504B, Costa Rica Rift, Rev. Geophys., 27, 79-102, 1989.

Bee, M., and L. D. Bibee, A seismic refraction study of Cretaceous oceanic lithosphere in the northwest Pacific Basin, Mar. Geophys. Res., 11, 239-261, 1989.

Berge, P., G. Fryer, and R. Wilkens, Velocity-porosity relationships in the upper oceanic crust: Theoretical considerations, J. Geophys. Res., 97, 15,239-15,254, 1992.

Beske-Diehl, S. J., Comment on "Magnetization of the oceanic crust: Thermoremanent magnetization or chemical remanent magnetization" by C. A. Raymond and J. L. LaBreque, J. Geophys. Res., 94, 3046-3048, 1989.

Bicknell, J. D., J. C. Sempere, and K. C. Macdonald, Tectonics of a fast spreading center: A deep-tow and Sea Beam survey on the East Pacific Rise at $19^{\circ} 30^{\prime} \mathrm{S}$, Mar. Geophys. Res., 9, 24-45, 1987.

Bideau, D., R. Hékinian, C. Bollinger, M. Constantin, E. Gracia, C. Guivel, B. Sichler, R. Apprioual, and R. Le Gall, Submersible investigation of highly contrasted magmatic activities recorded on two segments of the Mid-Atlantic Ridge near $34^{\circ} 52^{\prime} \mathrm{N}$ and $33^{\circ} 55^{\prime} \mathrm{N}$, InterRidge News, 5(1), 9-14, 1996.

Birch, F., The velocity of compressional waves in rocks to 10 kilobars-part 1, J. Geophys. Res., 65, 1083-1102, 1960.

Blakely, R. J., Statistical averaging of marine magnetic anomalies and the aging of oceanic crust, J. Geophys. Res., 88, 2289-2296, 1983.

Bleil, U., and N. Peterson, Variations in magnetization intensity and low-temperature titanomagnetite oxidation of ocean floor basalts, Nature, 301, 384-388, 1983. 
Boschetti, F., M. C. Dentith, and R. D. List, Inversion of seismic refraction data using genetic algorithms, Geophysics, 61, 1715-1727, 1996.

Bratt, S. R., and G. M. Purdy, Structure and variability of oceanic crust on the flanks of the East Pacific Rise between $11^{\circ}$ and $13^{\circ} \mathrm{N}, J$. Geophys. Res., 89, 6111-6125, 1984.

Bratt, S. R., and S. C. Solomon, Compressional and shear wave structure of the East Pacific Rise at $11^{\circ} 20^{\prime} \mathrm{N}$ : Constraints from three-component ocean bottom seismometer data, $J$. Geophys. Res., 89, 6095-6110, 1984.

Brocher, T. M., and R. A. Phinney, A ray parameter-intercept time spectral ratio method for seismic reflectivity analysis, J. Geophys. Res., 86, 7865-7873, 1981.

Bryan, W. B., and H. Fujimoto, Submersible observations of the WMARK ridge-transform intersection, MAR at 23 degrees N, Eos Trans. AGU (abstract), 75(44), 657, 1994.

Bryan, W. B., G. Thompson, and J. N. Ludden, Compositional variations in normal MORB from $22^{\circ}-25^{\circ} \mathrm{N}$ : Mid-Atlantic Ridge and Kane fracture zone, J. Geophys. Res., 86, 11,815$11,836,1981$.

Brysk, H., Numerical analysis of the 45-degree finite-difference equation for migration, Geophysics, 48, 532-542, 1983.

Burnett, M. S., D. W. Caress, and J. A. Orcutt, Tomographic image of the magma chamber at $12^{\circ} 50^{\prime} \mathrm{N}$ on the East Pacific Rise, Nature, 339, 206-208, 1989.

Calvert, A. J., Backscattered coherent noise and seismic reflection imaging of the oceanic crust: An example from the rift valley of the Mid-Atlantic Ridge at $23^{\circ} \mathrm{N}, J$. Geophys. Res., 102, 5119-5133, 1997.

Canales, J. P., and R. S. Detrick, Segment-scale crustal structure variations within the rift mountains of the Mid-Atlantic Ridge $\left(35^{\circ} \mathrm{N}\right)$, Annales Geophysicae, XXIII EGS General Assembly, 16(I), 292, 1998.

Cande, S. C., and D. V. Kent, Revised calibration of the geomagnetic polarity timescale for the Late Cretaceous and Cenozoic, J. Geophys. Res., 100, 6093-6095, 1995.

Cann, J. R., A model for oceanic crustal structure developed, Geophys. J. R. Astron. Soc., 39, 169-187, 1974.

Cannat, M., Emplacement of mantle rocks in the seafloor at mid-ocean ridges, J. Geophys. Res., 98, 4163-4172, 1993.

Cannat, M., V. Mamaloukas-Frangoulis, J.-M. Auzende, D. Bideau, E. Bonatti, J. Honnorez, Y. Lagabrielle, J. Malavieille, and C. Mevel, A geological cross-section of the Vema fracture zone transverse ridge, Atlantic Ocean, J. Geodynamics, 13, 97-118, 1991.

Carbotte, S. M., J. C. Mutter, and L. Xu, Contribution of volcanism and tectonism to axial flank morphology of the southern East Pacific Rise, $17^{\circ} 10^{\prime}-17^{\circ} 40^{\prime} \mathrm{S}$, from a study of layer 2A geometry, J. Geophys. Res., 102, 10,165-10,184, 1997. 
Caress, D. W., M. S. Burnett, and J. A. Orcutt, Tomographic image of the axial low-velocity zone at $12^{\circ} 50^{\prime} \mathrm{N}$ on the East Pacific Rise, J. Geophys. Res., 97, 9243-9263, 1992.

Carlson, R. L., Seismic velocities in the uppermost oceanic crust: Age dependence and the fate of layer 2A, J. Geophys. Res., 103, 7069-7077, 1998.

Cary, P. W., and C. H. Chapman, Automated 1-D waveform inversion of marine seismic refraction data, J. Geophys. Res., 93, 527-546, 1988.

Chadwick, W. W., R. W. Embley, and C. G. Fox, SeaBeam depth changes associated with recent lava flows, CoAxial segment, Juan de Fuca Ridge: Evidence for multiple eruptions between 1981-1993, Geophys. Res. Lett., 22, 167-170, 1995.

Chapman, C. H., A new method for computing synthetic seismograms, Geophys. J. $R$. Astron. Soc., 54, 481-518, 1978.

Chapman, C. H., Generalized radon transforms and slant stacks, Geophys. J. R. Astron. Soc., 66, 445-453, 1981.

Christenson, N. I., and M. H. Salisbury, Structure and composition of the lower oceanic crust, Rev. Geophys., 13, 57-86, 1975.

Christeson, G. L., G. M. Purdy, and G. J. Fryer, Structure of young upper crust at the East Pacific Rise near 9³0’N, Geophys. Res. Lett., 19, 1045-1048, 1992.

Christeson, G. L., G. M. Purdy, and G. J. Fryer, Seismic constraints on shallow crustal emplacement processes at the fast spreading East Pacific Rise, J. Geophys. Res., 99, 17,957-17,973, $1994 \mathrm{a}$.

Christeson, G. L., W. S. D. Wilcock, and G. M. Purdy, The shallow attenuation structure of the fast-spreading East Pacific Rise near $9^{\circ} 30^{\prime} \mathrm{N}$, Geophys. Res. Lett., 21, 321-324, 1994b.

Christeson, G. L., G. M. Kent, G. M. Purdy, and R. S. Detrick, Extrusive thickness variability at the East Pacific Rise, $9^{\circ}-10^{\circ} \mathrm{N}$ : Constraints from seismic techniques, J. Geophys. Res., 101, 2859-2973, 1996.

Christie, D. M., and J. M. Sinton, Evolution of abyssal lavas along propagating segments of the Galapagos spreading center, Earth Planet. Sci. Lett., 56, 321-335, 1981.

Claerbout, J. F., Fundamentals of geophysical data processing, McGraw-Hill Book Co., Inc., New York, 1976.

Collins, J. A., G. M. Purdy, and T. M. Brocher, Seismic velocity structure at Deep Sea Drilling Project Hole 504B, Panama Basin: Evidence for thin oceanic crust, J. Geophys. Res., 94, 9293-9302, 1989.

Cormier, M.-H., and K. C. Macdonald, East Pacific Rise $18^{\circ}-19^{\circ} \mathrm{S}$ : Asymmetric spreading and ridge reorientation by ultrafast migration of axial discontinuities, J. Geophys. Res., 99, 543-564, 1994. 
Cudrack, C. F., and R. M. Clowes, Crustal structure of Endeavour Kidge Segment, Juan de Fuca Ridge, from a detailed seismic refraction survey, J. Geophys. Res., 98, 6329-6349, 1993.

Davis, L., Handbook on genetic algorithms, Van Nostrand Reinhold, 1991.

DeMets, C., R. G. Gordon, D. F. Argus, and S. Stein, Current plate motions, Geophys. J. Int., 101, 425-478, 1990.

Detrick, R. S., P. Buhl, E. Vera, J. Mutter, J. Orcutt, J. Madsen, and T. Brocher, Multichannel seismic imaging of a crustal magma chamber along the East Pacific Rise, Nature, $326,35-42,1987$.

Detrick, R. S., J. C. Mutter, P. Buhl, and I. I. Kim, No evidence from multichannel reflection data for a crustal magma chamber in the MARK area on the Mid-Atlantic Ridge, Nature, $347,61-64,1990$.

Detrick, R. S., A. J. Harding, G. M. Kent, J. A. Orcutt, J. C. Mutter, and P. Buhl, Seismic structure of the southern East Pacific Rise, Science, 259, 499-503, 1993.

Detrick, R. S., J. Collins, R. Stephen, and S. Swift, In situ evidence for the nature of the seismic layer 2/3 boundary in oceanic crust, Nature, 370, 288-290, 1994.

Detrick, R. S., H. D. Needham, and V. Renard, Gravity anomalies and crustal thickness along the Mid-Atlantic Ridge between $33^{\circ} \mathrm{N}$ and $40^{\circ} \mathrm{N}, J$. Geophys. Res., 100, 3767-3787, 1995.

Detrick, R. S., J. Collins, G. Kent, J. Lin, D. Toomey, A. Barclay, E. Hooft, A. Hosford, and S. Hussenoeder, Mid-Atlantic Ridge Bullseye Experiment: A seismic investigation of segment-scale crustal heterogeneity at a slow-spreading ridge, InterRidge News, 6(1), 27-32, 1997.

Dewey, J. F., and W. S. F. Kidd, Geometry of plate accretion, Geol. Soc. Am. Bull., 88, 960-968, 1977.

Drijkoningen, G. G., and R. S. White, Seismic velocity structure of oceanic crust by inversion using genetic algorithms, Geophys. J. Int., 123, 653-664, 1995.

Duennebier, F. K., B. Lienert, R. Cessano, P. Anderson, and S. Mallick, Controlled source experiment at Hole 581C, Initial Rep. Deep Sea Drill. Proj., 88, 105-125, 1987.

Edwards, M. H., D. J. Fornari, A. Malinverno, W. B. F. Ryan, and J. Madsen, The regional fabric of the East Pacific Rise from $12^{\circ} 50^{\prime} \mathrm{N}$ to $15^{\circ} 10^{\prime} \mathrm{N}, J$. Geophys. Res., 96, 7995-8017, 1991.

Escartin, J., and J. Lin, Ridge offsets, normal faulting, and gravity anomalies of slow spreading ridges, J. Geophys. Res., 100, 6163-6177, 1995. 
Ewing, J. I., and G. M. Purdy, Upper crustal velocity structure in the ROSE area of the East Pacific Rise, J. Geophys. Res., 87, 8397-8402, 1982.

Fischer, K. M., and G. M. Purdy, Seismic amplitude modeling and the shallow crustal structure of the East Pacific Rise at $12^{\circ} \mathrm{N}$, J. Geophys. Res., 91, 14,006-14,014, 1986.

Fornari, D. J., M. R. Perfit, R. Batiza, and M. H. Edwards, Submersible transects across the East Pacific Rise crust and upper-flanks at $9^{\circ} 31^{\prime}-32^{\prime} \mathrm{N}$ : 1 . Observations of seafloor morphology and evidence for young off-axis volcanism, Eos Trans. AGU (abstract), 73(43), $525,1992$.

Forrest, S., Documentation for prisoners dilemma and norms programs that use the genetic algorithm, unpublished manuscript, University of Michigan, Ann Arbor.

Forsyth, D. W., Geophysical constraints on mantle flow and melt generation beneath midocean ridges, in Mantle Flow and Melt Generation at Mid-Ocean Ridges, edited by J. Phipps Morgan, D. K. Blackman, and J. M. Sinton, Geophysical Monograph No. 71, chapter 1, pp. 1-65, AGU, Washington, D.C., 1992.

Forsyth, D. W., and A. D. Chave, Experiment investigates magma in the mantle beneath mid-ocean ridges, Eos Trans. AGU, 75(46), 537-540, 1994.

Fowler, C. M. R., Crustal structure of the Mid-Atlantic ridge crest at $37^{\circ} \mathrm{N}$, Geophys. $J . R$. Astron. Soc., 47, 459-491, 1976.

Fowler, C. M. R., The Mid-Atlantic Ridge: Structure at $45^{\circ} \mathrm{N}$, Geophys. J. R. Astron. Soc., $54,167-183,1978$.

Fox, C. G., K. M. Murphy, and R. W. Embley, Automated display and statistical analysis of interpreted deep-sea bottom photographs, Marine Geology, 78, 199-216, 1987.

Fox, P. J., and J. B. Stroup, The plutonic foundation of the oceanic crust, in The Oceanic Lithosphere, edited by C. Emiliani, no. 7 in The Sea, chapter 4, pp. 119-218, John Wiley \& Sons, New York, 1981.

Fox, P. J., E. Schreiber, and J. J. Peterson, The geology of the oceanic crust: Compressional wave velocities of oceanic crust, J. Geophys. Res., 78, 5155-5172, 1973.

Francheteau, J., R. Hekinian, X. Le Pichon, D. Needham, P. Choukroune, P. Tapponnier, G. Bellaiche, and J. L. Cheminee, Transform fault and rift valley from bathyscaph and diving saucer, Science, 190, 108-116, 1975.

Francheteau, J., R. Armijo, J. L. Cheminée, R. Hekinian, P. Lonsdale, and N. Blum, 1 Ma East Pacific Rise oceanic crust and uppermost mantle exposed by rifting in Hess Deep, Earth Planet. Sci. Lett., 101, 281-295, 1990.

Francheteau, J., R. Armijo, J. L. Cheminée, R. Hekinian, P. Lonsdale, and N. Blum, Dyke complex of the East Pacific Rise exposed in the walls of Hess Deep and the structure of the upper oceanic crust, Earth Planet. Sci. Lett., 111, 109-121, 1992. 
Francis, T. J. G., and I. T. Porter, Median valley seismology: The Mid-Atlantic Ridge near $45^{\circ} \mathrm{N}$, Geophys. J. R. Astron. Soc., 34, 279-311, 1973.

Fujimoto, H., et al., A geophysical survey of the Mid-Atlantic Ridge around the western Kane transform fault, Eos Trans. AGU (abstract), 75(44), 656, 1994.

Gee, J., and D. V. Kent, Variations in layer $2 \mathrm{~A}$ thickness and the origin of the central anomaly magnetic high, J. Geophys. Res., 21, 297-300, 1994.

Gillis, K. M., and K. Sapp, Distribution of porosity in a section of upper oceanic crust exposed in the Troodos Ophiolite, J. Geophys. Res., 102, 10,133-10,149, 1997.

Goldberg, D. E., Genetic algorithms in search, optimization $\mathcal{O}$ machine learning, AddisonWesley, Reading, MA, 1989.

Gregg, T. K. P., and J. H. Fink, Quantification of submarine lava-flow morphology through analog experiments, Geology, 23, 73-76, 1995.

Gregg, T. K. P., D. J. Fornari, M. R. Perfit, R. M. Haymon, and J. H. Fink, Rapid emplacement of a mid-ocean ridge lava flow on the East Pacific Rise at $9^{\circ} 46^{\prime}-52^{\prime} \mathrm{N}$, Earth Planet. Sci. Lett., 144, 1-7, 1996.

Grevemeyer, I., and W. Weigel, Seismic velocities of the uppermost igneous crust versus age, Geophys. J. Int., 124, 631-635, 1996.

Grevemeyer, I., and W. Weigel, Increase of seismic velocities in upper oceanic crust: The "superfast" spreading East Pacific Rise at $14^{\circ} 14^{\prime}$ S, Geophys. Res. Lett., 24, 217-220, 1997.

Grevemeyer, I., N. Kaul, H. Villinger, and W. Weigel, Hyrdothermal circulation and the seismic structure of upper oceanic crust: Results from the EXCO-cruise, InterRidge News, 6, 27-30, 1997.

Grindlay, N. R., P. J. Fox, and P. R. Vogt, Morphology and tectonics of the Mid-Atlantic Ridge $\left(25^{\circ}-27^{\circ} 30^{\prime} \mathrm{S}\right)$ from Sea Beam and magnetic data, J. Geophys. Res., 97, 6983-7010, 1992.

Guspi, F., Frequency-domain reduction of potential field measurements to a horizontal plane, Geoexploration, 24, 87-98, 1987.

Hansen, R. O., and Y. Miyazaki, Continuation of potential fields between arbitrary surfaces, Geophysics, 49, 787-795, 1984.

Harding, A. J., and M. E. Kappus, Genetic algorithm waveform inversion of East Pacific Rise refraction data, unpublished manuscript, Scripps Institution of Oceanography.

Harding, A. J., J. A. Orcutt, M. E. Kappus, E. E. Vera, J. C. Mutter, P. Buhl, R. S. Detrick, and $\mathrm{T}$. Brocher, The structure of young oceanic crust at $13^{\circ} \mathrm{N}$ on the East Pacific Rise from expanding spread profiles, J. Geophys. Res., 94, 12,163-12,196, 1989. 
Harding, A. J., G. M. Kent, and J. A. Orcutt, A multichannel seismic investigation of upper crustal structure at $9^{\circ} \mathrm{N}$ on the East Pacific Rise: Implications for crustal accretion, $J$. Geophys. Res., 98, 13,925-13,944, 1993.

Harrison, C. G. A., Marine magnetic anomalies-the origin of the stripes, Ann. Rev. Earth Planet. Sci., 15, 505-543, 1987.

Haymon, R. M., D. J. Fornari, M. H. Edwards, S. Carbotte, D. Wright, and K. C. Macdonald, Hydrothermal vent distribution along the East Pacific Rise crest $\left(9^{\circ} 09^{\prime}-54^{\prime} \mathrm{N}\right)$ and its relationship to magmatic and tectonic processes on fast spreading mid-ocean ridges, Earth Planet. Sci. Lett., 104, 513-534, 1991.

Haymon, R. M., et al., Volcanic eruption of the mid-ocean ridge along the East Pacific Rise crest at $9^{\circ} 45-52^{\prime} \mathrm{N}$ : Direct submersible observations of the seafloor phenomena associated with an eruption in April, 1991, Earth Planet. Sci. Lett., 119, 85-101, 1993.

Henry, M., J. A. Orcutt, and R. L. Parker, A new method for slant stacking refraction data, Geophys. Res. Lett., 7, 1073-1076, 1980.

Herron, T. J., Lava Hlow layer-East Pacific Rise, Geophys. Res. Lett., 9, 17-20, 1982.

Holland, J. H., Adaptation in natural and artificial systems, The University of Michigan Press, Ann Arbor, 1975.

Hooft, E. E., and R. S. Detrick, The role of density in the accumulation of basaltic melts at mid-ocean ridges, Geophys. Res. Lett., 20, 423-426, 1993.

Hooft, E. E. E., H. Schouten, and R. S. Detrick, Constraining crustal emplacement processes from the variation in seismic layer $2 \mathrm{~A}$ thickness at the East Pacific Rise, Earth Planet. Sci. Lett., 142, 289-309, 1996.

Hooft, E. E. E., R. S. Detrick, and G. M. Kent, Seismic structure and indicators of magma budget along the southern East Pacific Rise, J. Geophys. Res., 102, 27,319-27,340, 1997a.

Hooft, E. E. E., R. S. Detrick, D. R. Toomey, J. A. Collins, and J. Lin, MAR Bullseye Seismic Experiment: Seismic crustal structure along the axial valley of three contrasting spreading segments, $33.5-35^{\circ} \mathrm{N}$, Eos Trans. AGU (abstract), 78(46), 670, 1997b.

Hosford, A., R. Detrick, J. Collins, J. Lin, and D. Toomey, Seismic structure and gravity models of inside-corner crust at $35^{\circ} \mathrm{N}$ on the Mid-Atlantic Ridge, Eos Trans. $A G U$ (abstract), 79(17), 335, 1998.

Houtz, R., and J. I. Ewing, Upper crustal structure as a function of plate age, J. Geophys. Res., 81, 2490-2498, 1976.

Houtz, R. E., Seismic properties of layer 2A in the Pacific, J. Geophys. Res., 81, 6321-6331, 1976. 
Hussenoeder, S. A., M. A. Tivey, and H. Schouten, Direct inversion of potential fields from an uneven track with application to the Mid-Atlantic Ridge, Geophys. Res. Lett., 22, 3131-3135, 1995.

Hussenoeder, S. A., J. A. Collins, G. M. Kent, R. S. Detrick, and the TERA Group, Seismic analysis of the axial magma chamber reflector along the southern East Pacific Rise from conventional reflection profiling, J. Geophys. Res., 101, 22,087-22,105, 1996a.

Hussenoeder, S. A., M. A. Tivey, H. Schouten, and R. C. Searle, Near-bottom magnetic survey of the Mid-Atlantic Ridge axis, $24^{\circ}-24^{\circ} 40^{\prime} \mathrm{N}$ : Implications for crustal accretion at slow spreading ridges, J. Geophys. Res., 101, 22,051-22,069, $1996 \mathrm{~b}$.

Hyndman, R. D., and M. J. Drury, The physical properties of oceanic basement rocks from deep drilling on the Mid-Atlantic Ridge, J. Geophys. Res., 81, 4042-4052, 1976.

International Association of Geomagnetism and Aeromagnetism (IAGA) Working Group I, International Geomagnetic Reference Field Revision, IAGA News, 26, 87-92, 1987.

Jacobson, R. S., Impact of crustal evolution on changes of the seismic properties of the uppermost ocean crust, Rev. Geophys., 30, 23-42, 1992.

Johnson, H. P., and T. Atwater, Magnetic study of basalts from the Mid-Atlantic Ridge, lat. $37^{\circ} \mathrm{N}$, Geol. Soc. Am. Bull., 88, 637-647, 1977.

Johnson, H. P., and R. T. Merrill, Low-temperature oxidation of a titanomagnetite and the implications for paleomagnetism, J. Geophys. Res., 78, 4938-4949, 1973.

Johnson, H. P., and J. E. Pariso, The effect of hydrothermal alteration on the magnetic properties of oceanic crust: Results from drillholes CY-2 and CY-2a, Cyprus Study Project, in Cyprus Crustal Study Project: Initial Reports, Holes CY-2 and 2a, edited by P. T. Robinson, I. L. Gibson, and A. Panayiotou, pp. 283-293, Geol. Surv. Can., 1987.

Johnson, H. P., D. V. Kent, M. A. Tivey, J. S. Gee, R. L. Larson, and R. W. Embley, Conference on the Magnetization of the Oceanic Crust steers future research, Eos Trans. $A G U$, 78(19), 199-200, 1997.

Juhlin, C., and R. Young, Implications of thin layers for amplitude variation with offset (AVO) studies, Geophysics, 58, 1200-1204, 1993.

Kallweit, R. S., and L. C. Wood, The limits of resolution of zero-phase wavelets, Geophysics, 47, 1035-1046, 1982.

Kappel, E. S., and W. B. Ryan, Volcanic episodicity and a non-steady state rift-valley along Northeast Pacific spreading centers: Evidence from SeaMARC I, J. Geophys. Res., 91, 13,925-13,940, 1986.

Kappus, M. E., A. J. Harding, and J. A. Orcutt, A comparison of tatl-p transform methods, Geophysics, 55, 1202-1215, 1990. 
Kappus, M. E., A. J. Harding, and J. A. Orcutt, A baseline for upper crustal velocity variations along the East Pacific Rise at $13^{\circ} \mathrm{N}, J$. Geophys. Res., 100, 6143-6161, 1995.

Karson, J. A., and H. J. B. Dick, Tectonics of ridge-transform intersections at the Kane fracture zone, Mar. Geophys. Res., 6, 51-98, 1983.

Karson, J. A., S. D. Hurst, and P. Lonsdale, Tectonic rotations of dikes in fast-spread oceanic crust exposed near Hess Deep, Geology, 20, 685-688, 1992.

Keen, C., and C. Tramontini, A seismic refraction survey on the Mid-Atlantic Ridge, Geophys. J. R. Astron. Soc., 20, 473-491, 1970.

Kennett, B. L. N., Seismic waves in a stratified half space, Geophys. J. Roy. Astr. Soc., 61, 1-10, 1980.

Kennett, B. L. N., Seismic wave propagation in a stratified media, Cambridge Univ. Press, 1983.

Kent, G. M., A. J. Harding, and J. A. Orcutt, Evidence for a smaller magma chamber beneath the East Pacific Rise at $9^{\circ} 30^{\prime} \mathrm{N}$, Nature, 344, 650-653, 1990.

Kent, G. M., A. J. Harding, and J. A. Orcutt, Distribution of magma beneath the East Pacific Rise between the Clipperton transform and the $9^{\circ} 17^{\prime} \mathrm{N}$ deval from forward modeling of common depth point data, J. Geophys. Res., 98, 13,945-13,969, 1993.

Kent, G. M., A. J. Harding, J. A. Orcutt, R. S. Detrick, J. C. Mutter, and P. Buhl, Uniform accretion of oceanic crust south of the Garrett transform at $14^{\circ} 15^{\prime} \mathrm{S}$ on the East Pacific Rise, J. Geophys. Res., 99, 9097-9116, 1994.

Kidd, R. G. W., A model for the process of formation of the upper oceanic crust, Geophys. J. R. Astron. Soc., 50, 149-183, 1977.

Kinoshita, H., T. Furuta, and J. Pariso, 13. Downhole magnetic field measurements and paleomagnetism, Hole 504B, Costa Rica Ridge, in Proceed. Ocean. Drill. Prog., Sci. Res., Vol. 111, pp. 147-156, 1989.

Kleinrock, M. C., Capabilities of some systems used to survey the deep-sea floor, vol. 2Hard Minerals of CRC Handbook of Geophysical Exploration at Sea, chapter 2, pp. 35-86, 2nd ed., CRC Press, 1992.

Kleinrock, M. C., et al., A model for the process of formation of the upper oceanic crust, Initial Rep. Deep Sea Drill. Proj., 158, 15-21, 1996.

Klitgord, K. D., Sea-floor spreading: The central anomaly magnetization high, Earth Planet. Sci. Lett., 29, 201-209, 1976.

Klitgord, K. D., and H. Schouten, Plate kinematics of the central Atlantic, in The Western North Atlantic Region, edited by P. R. Vogt and B. E. Tucholke, vol. M of The Geology of North America, chapter 22, pp. 351-378, Geological Society of America, Boulder, Colorado, 1986. 
Kuo, B. Y., and D. W. Forsyth, Gravity anomaly of the ridge transform system in the south Atlantic between 31 and $34.5^{\circ} \mathrm{S}$ : Upwelling centers and variation in crustal thickness, Mar. Geophys. Res., 10, 205-232, 1988.

Langmuir, C. H., J. F. Bender, and R. Batiza, Petrological and tectonic segmentation of the East Pacific Rise, 530'-14'30`N, Nature, 322, 422-429, 1986.

Lawson, K., R. C. Searle, J. A. Pearce, P. Browning, and P. Kempton, Detailed volcanic geology of the MARNOK area, Mid-Atlantic Ridge north of Kane transform, in Tectonic, Magmatic, Hydrothermal and Biological Segmentation of Mid-Ocean Ridges, edited by C. J. MacLeod, P. A. Tyler, and C. L. Walker, Geol. Soc. of Am. Spec. Publ. No. 118, pp. 61-102, The Geological Society of America, Boulder, Colorado, 1996.

LeDouaran, S., H. D. Needham, and J. Francheteau, Pattern of opening rates along the axis of the Mid-Atlantic Ridge, Nature, 300, 254-257, 1982.

Lin, J., and J. Phipps Morgan, The spreading rate dependence of three-dimensional midocean ridge gravity structure, Geophys. Res. Lett., 19, 13-16, 1992.

Lin, J., G. M. Purdy, H. Schouten, J. C. Sempéré, and C. Zervas, Evidence from gravity data for focused magmatic accretion along the Mid-Atlantic Ridge, Nature, 344, 627-632, 1990.

Little, S. A., and R. A. Stephen, Costa Rica Rift borehole seismic experiment, Deep Sea Drilling Project Hole 504B, leg 92, Initial Rep. Deep Sea Drill. Proj., 83, 517-528, 1985.

Lonsdale, P., Segmentation of the Pacific-Nazca spreading center, $1^{\circ} \mathrm{N}-20^{\circ} \mathrm{S}, \mathrm{J}$. Geophys. Res., 94, 12,197-12,225, 1989.

Luyendyk, B. P., and K. C. Macdonald, A geological transect across the crest of the East Pacific Rise at $21^{\circ} \mathrm{N}$ latitude made from the deep submersible ALVIN, Mar. Geophys. Res., 7, 467-488, 1985.

Macdonald, K. C., Near-bottom magnetic anomalies, asymmetric spreading, oblique spreading, and tectonics of the Mid-Atlantic Ridge near lat $37^{\circ} \mathrm{N}$, Geol. Soc. Am. Bull., 88 , $541-555,1977$.

Macdonald, K. C., The crest of the Mid-Atlantic Ridge: Models for crustal generation processes and tectonics, in The Western North Atlantic Region, edited by P. R. Vogt and B. E. Tucholke, vol. M of The Geology of North America, chapter 4, pp. 51-68, The Geological Society of America, Boulder, Colorado, 1986.

Macdonald, K. C., Tectonic and magmatic processes on the East Pacific Rise, in The Eastern Pacific Ocean and Hawaii, edited by E. L. Winter, D. M. Hussong, and R. W. Decker, vol. N of The Geology of North America, chapter 6, pp. 93-110, The Geological Society of America, Boulder, Colorado, 1989. 
Macdonald, K. C., and P. J. Fox, The axial summit graben and cross-sectional shape of the East Pacific Rise as indicators of axial magma chambers and recent volcanic eruptions, Earth Planet. Sci. Lett., 88, 119-131, 1988.

Macdonald, K. C., and B. P. Luyendyk, Deep-tow studies of the structure of the MidAtlantic Ridge crest near lat $37^{\circ}$ N, Geol. Soc. Am. Bull., 88, 621-636, 1977.

Macdonald, K. C., J. C. Sempéré, and P. J. Fox, East Pacific Rise from Sequeiros to Orozco fracture zones: Along-strike continuity of axial neovolcanic zone and structure and evolution of overlapping spreading centers, J. Geophys. Res., 89, 6049-6069, 1984.

Macdonald, K. C., P. J. Fox, L. J. Perram, M. F. Eisen, R. M. Haymon, S. P. Miller, S. M. Carbotte, M.-H. Cormier, and A. N. Shor, A new view of the mid-ocean ridge from the behaviour of ridge-axis discontinuities, Nature, 335, 217-225, 1988.

Macdonald, K. C., R. Haymon, and A. Shor, A $220 \mathrm{~km}^{2}$ recently erupted lava field on the East Pacific Rise near lat $8^{\circ} \mathrm{S}$, Geology, 17, 212-216, 1989.

Mallick, S., Model-based inversion of amplitude-variations with offset data using a genetic algorithm, Geophysics, 60, 939-954, 1995.

Manghnani, M. H., H. Sato, and C. S. Rai, Ultrasonic velocity and attenuation measurements on basaltic melts to $1500^{\circ} \mathrm{C}$ : Role of composition and structure in the viscoelastic properties, J. Geophys. Res., 91, 9333-9342, 1986.

Marsh, B. D., Magma chambers, Ann. Rev. Earth Planet. Sci., 17, 439-474, 1989.

McClain, J. S., and C. A. Atallah, The structure of young oceanic crust near a very fast spreading ridge, Geophys. Res. Lett., 12, 689-692, 1985.

McClain, J. S., J. A. Orcutt, and M. Burnett, The East Pacific Rise in cross section: A seismic model, J. Geophys. Res., 90, 8627-8639, 1985.

McDonald, M. A., S. C. Webb, J. A. Hildebrand, B. D. Cornuelle, and C. G. Fox, Seismic structure and anisotropy of the Juan de Fuca Ridge at $45^{\circ} \mathrm{N}$, J. Geophys. Res., 99, 4857-4873, 1994.

Meissner, R., and E. Meixner, Deformation of seismic wavelets by thin layers and layered boundaries, Geophysical Prospecting, 17, 1-27, 1969.

Mies, P. J., Aeromagnetic compensation, Masters thesis, Colorado School of Mines, 1986.

Moores, E. M., Origin and emplacement of ophiolites, Rev. Geophys., 20, 735-760, 1982.

Murase, T., and A. R. McBirney, Properties of some common igneous rocks and their melts at high temperatures, Geol. Soc. Am. Bull., 84, 3563-3592, 1973.

Mutter, J. C., and J. A. Karson, Structural processes at slow-spreading ridges, Science, 257, 627-634, 1992. 
Mutter, J. C., S. M. Carbotte, W. Su, L. Xu, P. Buhl, R. S. Detrick, G. M. Kent, J. A. Orcutt, and A. J. Harding, Seismic images of active magma systems beneath the East Pacific Rise between $17^{\circ} 05^{\prime}$ to $17^{\circ} 35^{\prime} \mathrm{S}$, Science, 268, 391-395, 1995.

Naar, D. F., and R. N. Hey, Recent Pacific-Easter-Nazca plate motions, in Evolution of Mid-Ocean Ridges, edited by J. M. Sinton, no. 57 in Geophysical Monograph, chapter 1, pp. 9-30, AGU, Washington, D.C., 1989.

Needham, H. D., et al., The crest of the Mid-Atlantic Ridge between $40^{\circ}$ and $15^{\circ} \mathrm{N}$ : Very broad swath mapping with the EM12 echo sounding system, Eos Trans. AGU (abstract), 72(44), 470, 1991.

Neumann, G. A., and D. W. Forsyth, The paradox of the axial profile: Isostatic compensation along the axis of the Mid-Atlantic Ridge?, J. Geophys. Res., 98, 17,891-17,910, 1993.

Nicolas, A., Structure of Ophiolites and Dynamics of Oceanic Lithosphere, Kluwer, Dordrecht, 1989.

Nolte, B., and L. N. Frazer, Vertical seismic profile inversion with genetic algorithms, Geophys. J. Int., 117, 162-178, 1994.

O'Connell, R. J., and B. Budiansky, Measures of dissipation in viscoelastic media, Geophys. Res. Lett., 5, 5-8, 1978.

Pálmason, G., A continuum model of crustal generation in Iceland; Kinematic aspects, $J$. Geophys., 47, 7-18, 1980.

Pariso, J. E., and H. P. Johnson, 14. magnetic properties and oxide petrography of the sheeted dike complex in Hole 504B, in Proceed. Ocean. Drill. Prog., Sci. Res., Vol. 111, pp. 159-167, 1989.

Parker, R. L., The rapid calculation of potential anomalies, Geophys. J. R. Astron. Soc., $31,447-455,1972$.

Parker, R. L., and S. P. Huestis, The inversion of magnetic anomalies in the presence of topography, J. Geophys. Res., 79, 1587-1593, 1974.

Parker, R. L., and K. D. Klitgord, Magnetic upward continuation from an uneven track, Geophysics, 37, 662-668, 1972.

Parmentier, E. M., and J. Phipps Morgan, Spreading rate dependence of three-dimensional structure in oceanic spreading centers, Nature, 348, 325-328, 1990.

Perfit, M. R., D. J. Fornari, R. Batiza, and M. H. Edwards, Submersible transects across the East Pacific Rise crust and upper-flanks at $9^{\circ} 31^{\prime}-32^{\prime} \mathrm{N}$ : 2. Small-scale spatial variations in lava geochemistry and implications for temporal variability, Eos Trans. AGU (abstract), 73(43), 525, 1992. 
Pezard, P. A., Electrical properties of mid-ocean ridge basalt and implications for the structure of the upper oceanic crust in Hole 504B, J. Geophys. Res., 95, 9237-9264, 1990.

Phipps Morgan, J., and Y. J. Chen, The genesis of oceanic crust: Magama injection, hydrothermal circulation, and crustal flow, J. Geophys. Res., 98, 6283-6297, 1993.

Phipps Morgan, J., A. J. Harding, J. A. Orcutt, G. M. Kent, and Y. J. Chen, An observational and theoretical synthesis of magma chamber geometry and crustal genesis along a mid-ocean spreading center, in Magmatic Systems, edited by M. Ryan, chap. 7, pp. 139-178, Academic Press, San Diego, Calif., 1994.

Pilkington, M., and W. E. S. Urquhart, Reduction of potential field data to a horizontal plane, Geophysics, 55, 549-555, 1990.

Pockalny, R. A., A. Smith, and P. Gente, Spatial and temporal variability of crustal magnetization of a slowly spreading ridge: Mid-Atlantic Ridge $\left(20^{\circ}-24^{\circ}\right)$, Mar. Geophys. Res., 17, 301-320, 1995.

Poehls, K. A., Seismic refraction on the Mid-Atlantic Ridge at $37^{\circ} \mathrm{N}, J$. Geophys. Res., 79 , 3370-3373, 1974.

Poujol, M., and R. S. Jacobson, Using inverse theory and Seabeam bathymetry to improve seismic data corrections, Mar. Geophys. Res., 9, 311-332, 1988.

Prévot, M., and S. Grommé, Intensity of magnetization of subaerial and submarine basalts and its possible change with time, Geophys. J. R. Astron. Soc., 40, 207-224, 1975.

Purdy, G. M., The variability in seismic structure of layer 2 near the East Pacific Rise at $12^{\circ} \mathrm{N}, J$. Geophys. Res., 87, 8403-8416, 1982.

Purdy, G. M., New observations of the shallow seismic structure of young oceanic crust, $J$. Geophys. Res., 92, 9351-9362, 1987.

Purdy, G. M., and R. S. Detrick, Crustal structure of the Mid-Atlantic Ridge at $23^{\circ} \mathrm{N}$ from seismic refraction studies, J. Geophys. Res, 91, 3739-3762, 1986.

Purdy, G. M., J.-C. Sempéré, H. Schouten, D. L. DuBois, and R. L. Goldsmith, Bathymetry of the Mid-Atlantic Ridge, $24^{\circ}-31^{\circ} \mathrm{N}$ : A map series, Mar. Geophys. Res., 12, 247-252, 1990.

Purdy, G. M., D. R. Toomey, C. J. Wolfe, A. H. Barclay, and S. C. Solomon, FARA microearthquake experiments I: Observations on contrasting segments of the Mid-Atlantic Ridge, Eos Trans. AGU (abstract), 74(43), 601, 1993.

Raitt, R. W., The crustal rocks, vol. 3 of The Sea, chapter 6, pp. 85-102, Wiley-Interscience, New York, 1963.

Raymond, C. A., and J. L. LaBrecque, Magnetization of oceanic crust: Thermoremanent magnetization of chemical remanent magnetization?, J. Geophys. Res., 92, 8077-8088, 1987. 
Renard, V., R. Hekinian, J. Francheteau, R. D. Ballard, and H. Backer, Submersible observations at the axis of the ultra-fast spreading East Pacific Rise $17^{\circ} 30^{\circ}$ to $21^{\circ} 30^{\prime} \mathrm{S}$, Earth Planet. Sci. Lett., 75, 339-353, 1985.

Rohr, K. M. M., B. Milkereit, and C. J. Yorath, Asymmetric deep crustal structure across the Juan de Fuca Ridge, Geology, 16, 533-537, 1988.

Rommevaux, C., C. Deplus, P. Patriat, and J.-C. Sempéré, Three-dimensional gravity study of the Mid-Atlantic Ridge: Evolution of the segmentation between $28^{\circ}$ and $29^{\circ} \mathrm{N}$ during the last 10 m.y., J. Geophys. Res., 99, 3015-3029, 1994.

Rowlett, H., K. McCany, and G. Carpenter, An ocean bottom seismometer (OBS) refraction profile on the Mid-Atlantic Ridge at $13^{\circ} \mathrm{S}$, Eos Trans. AGU (abstract), 55(4), 294, 1974.

Ryan, M. P., Neutral buoyancy and the mechanical evolution of magmatic systems, in Magmatic Processes: Physiochemical Principles, Special Publication of the Geochemical Society, 1, edited by B. O. Mysen, pp. 259-287, Geochemical Society, 1987.

Ryan, M. P., Neutral buoyancy and the structure of mid-ocean ridge magma reservoirs, $J$. Geophys. Res., 98, 22,321-22,338, 1993.

Sambridge, M., and G. Drijkoningen, Genetic algorithms in seismic waveform inversion, Geophys. J. Int., 109, 323-342, 1992.

Sato, H., and M. H. Manghnani, Ultrasonic measurements of $V_{p}$ and $Q_{p}$ : Relaxation spectrum of complex modulus on basalt melts, Phys. Earth Planet. Inter., 41, 18-33, 1985.

Sato, H., I. S. Sacks, T. Murase, G. Muncill, and H. Fukuyama, Attenuation of compressional waves in peridotite measured as a function of temperature at $200 \mathrm{MPa}$, Pure Appl. Geophys., 128, 433-447, 1988.

Sato, H., I. S. Sacks, T. Murase, G. Muncill, and H. Fukuyama, $Q_{p}$-melting temperature relation in peridotite at high pressure and temperature: Attenuation mechanism and implications for the mechanical properties of the upper mantle, J. Geophys. Res., 94, $10,647-10,661,1989$.

Scheirer, D. S., and K. C. Macdonald, The variation in cross-sectional area of the axial ridge of the East Pacific Rise: Evidence for the magmatic budget of a fast-spreading center, $J$. Geophys. Res., 98, 7871-7885, 1993.

Scheirer, D. S., K. C. Macdonald, D. W. Forsyth, S. P. Miller, D. J. Wright, M.-H. Cormier, and C. M. Weiland, A map series of the southern East Pacific Rise and its flanks, $15^{\circ} \mathrm{S}$ to $19^{\circ} \mathrm{S}$, Mar. Geophys. Res., 18, 1-12, 1996.

Scheirer, D. S., D. W. Forsyth, M.-H. Cormier, and K. C. Macdonald, Shipboard geophysical indications of asymmetry and melt production beneath the East Pacific Rise near the MELT experiment, Science, 280, 1221-1224, 1998. 
Schilling, J. G., M. Zajac, R. Evans, T. Johnston, W. White, J. D. Devine, and R. Kingsley, Petrologic and geochemical variation along the Mid-Atlantic Ridge from $29^{\circ} \mathrm{N}$ to $73^{\circ} \mathrm{N}$, Am. J. Sci., 283, 510-586, 1982.

Schmeling, H., Numerical models on the influence of partial melt on elastic, anelastic and electrical properties of rocks, part I, Elasticity and anelasticity, Phys. Earth Planet. Int., $41,34-57,1985$.

Schmincke, H.-U., M. Rautenschlein, P. T. Robinson, and J. M. Mehegan, Troodos extrusive series of Cyprus: A comparison with oceanic crust, Geology, 11, 405-409, 1983.

Schouten, H., and C. R. Denham, Modeling the oceanic magnetic source layer, in Deep drilling results in the Atlantic Ocean: Ocean crust, edited by M. Talwani, C. G. Harrison, and D. E. Hayes, pp. 151-159, AGU, 1979.

Schouten, H., and K. McCamy, Filtering marine magnetic anomalies, J. Geophys. Res., 77, 7089-7099, 1972.

Schouten, H., M. A. Tivey, D. J. Fornari, and J. R. Cochran, The central anomaly magnetic high: Constraints on volcanic construction of seismic layer $2 \mathrm{~A}$ at a fast spreading midocean ridge, the EPR at $9^{\circ} 30^{\prime}-50^{\prime} \mathrm{N}$, submitted to Earth Planet. Sci. Lett., 1998.

Schreiber, E., and P. J. Fox, Compressional wave velocities and mineralogy of fresh basalts from the Famous area and the Oceanographer fracture zone and the texture of layer $2 \mathrm{~A}$ of the oceanic crust, J. Geophys. Res., 81, 4071-4076, 1976.

Searle, R. C., P. A. Cowie, N. C. Mitchell, S. Allerton, C. J. MacLeod, J. Escartin, S. M. Russel, P. A. Slootweg, and T. Tanaka, Fault structure and detailed evolution of a slow spreading ridge segment: The Mid-Atlantic Ridge at $29^{\circ} \mathrm{N}$, Earth Planet. Sci. Lett., 154, 167-183, 1998.

Sempéré, J.-C., High-magnetization zones near spreading center discontinuities, Earth Planet. Sci. Lett., 107, 389-405, 1991.

Sempéré, J.-C., and K. C. Macdonald, Magnetic and petrologic consequences of spreading center discontinuities and the surficial boundaries of spreading cells beneath mid-ocean ridges, Eos Trans. $A G U$ (abstract), 67(16), 359, 1986.

Sempéré, J.-C., G. M. Purdy, and H. Schouten, Segmentation of the Mid-Atlantic Ridge between $24^{\circ} \mathrm{N}$ and $30^{\circ} 40^{\prime} \mathrm{N}$, Nature, 344, 427-431, 1990.

Sempéré, J.-C., H. S. Brown, J. Lin, H. Schouten, and G. M. Purdy, Segmentation and morphotectonic variations along a slow-spreading center: The Mid-Atlantic Ridge $\left(24^{\circ} 00^{\prime} \mathrm{N}\right.$ and $\left.30^{\circ} 40^{\prime} \mathrm{N}\right)$, Mar. Geophys. Res., 15, 153-200, 1993.

Sen, M. K., and P. L. Stoffa, Rapid sampling of model space using genetic algorithms: Examples from seismic waveform inversion, Geophys. J. Int., 108, 281-292, 1992. 
Sen, M. K., and P. L. Stoffa, Global optimization methods in geophysical inversion, vol. 4 of Advances in exploration geophysics, Elsevier, New York, 1995.

Severinghaus, J. P., and K. C. Macdonald, High inside corners at ridge-transform intersections, Mar. Geophys. Res., 9, 353-367, 1988.

Shipboard Scientific Party, Site 395: $23^{\circ}$ N, Mid-Atlantic Ridge, Initial Rep. Deep Sea Drill. Proj., 45, 131-264, 1979.

Shor Jr., G. G., H. W. Menard, and R. S. Raitt, Structure of the Pacific Basin, vol. 4 of The Sea, part II, pp. 3-27, Wiley-Interscience, New York, 1970.

Singh, S. C., G. M. Kent, J. S. Collier, A. J. Harding, and J. A. Orcutt, Melt to mush variations in crustal magma properties along the ridge crest of the southern East Pacific Rise, Nature, in press, 1998.

Sinha, M. C., and K. E. Louden, The Oceanographer fracture zone - I. Crustal structure from seismic refraction studies, Geophys. J. R. Astron. Soc., 75, 713-736, 1983.

Sinton, J. M., and R. S. Detrick, Mid-ocean ridge magma chambers, J. Geophys. Res., 97, 197-216, 1992.

Sinton, J. M., S. M. Smaglik, J. J. Mahoney, and K. C. Macdonald, Magmatic processes at superfast spreading mid-ocean ridges: Glass compositional variations along the East Pacific Rise $13^{\circ}-23^{\circ} \mathrm{S}$, J. Geophys. Res., 96, 6133-6155, 1993.

Smallwood, J. R., and R. S. White, Crustal accretion at the Reykjanes Ridge, $61^{\circ}-62^{\circ} \mathrm{N}$, J. Geophys. Res., 103, 5185-5201, 1998.

Smith, D. K., and J. R. Cann, Hundreds of small volcanoes on the median valley floor of the Mid-Atlantic Ridge at 24-30 N, Nature, 348, 152-155, 1990.

Smith, D. K., and J. R. Cann, The role of seamount volcanism in crustal construction at the Mid-Atlantic Ridge $\left(24^{\circ}-30^{\circ} \mathrm{N}\right)$, J. Geophys. Res., 97, 1645-1658, 1992.

Smith, D. K., M. A. Tivey, H. Schouten, and J. C. Cann, Tracking the active spreading axis along $80 \mathrm{~km}$ of the Mid-Atlantic Ridge, submitted to J. Geophys. Res., 1998.

Smith, G. M., and S. K. Banerjee, Magnetic structure of the upper kilometer of the marine crust at Deep Sea Drilling Project Hole 504B, eastern Pacific Ocean, J. Geophys. Res., $91,10,337-10,354,1986$.

Solomon, S. C., and D. R. Toomey, The structure of mid-ocean ridges, Ann. Rev. Earth Planet. Sci., 20, 329-364, 1992.

Sparks, D. W., and M. Parmentier, The generation and migration of melt beneath oceanic spreading centers, in Magmatic Systems, edited by M. P. Ryan, no. 57 in International geophysics series, pp. 55-76, Academic Press, New York, 1994. 
Spiegelman, M., Flow in deformable porous media, 1, Simple analysis, J. Fluid Mech., 247, 17-38, 1993.

Spudich, P., and J. Orcutt, Petrology and porosity of an oceanic crustal site: Results from wave form modeling of seismic refraction data, J. Geophys. Res., 85, 1409-1433, 1980.

Stephen, R. A., and A. J. Harding, Travel time analysis of borehole seismic data, J. Geophys. Res., 88, 8289-8298, 1983.

Stoffa, P. L., and M. K. Sen, Nonlinear multiparameter optimization using genetic algorithms: Inversion of plane-wave seismograms, Geophysics, 56, 1794-1810, 1991.

Stoffa, P. L., J. T. Fokkema, R. M. de Luna Friere, and W. P. Kessinger, Split-step Fourier migration, Geophysics, 55, 410-421, 1990.

Swift, B. A., and H. P. Johnson, Magnetic properties of the Bay of Islands ophiolite suite and implications for the magnetization of oceanic crust, J. Geophys. Res., 89, 3291-3308, 1984.

Talwani, M., and J. R. Heirtzler, Computation of magnetic anomalies caused by twodimensional bodies of arbitrary shape, in Computers in the mineral industries, edited by G. A. Parks, no. 9 in Geological Sciences, part 1, pp. 464-480, Stanford Univerity Publishers, 1964.

Talwani, M., C. C. Windisch, and M. G. Langseth Jr., Reykjanes Ridge crest: A detailed geophysical study, J. Geophys. Res., 76, 473-517, 1971.

Thibaud, R., P. Gente, and M. Maia, A systematic analysis of the morphology and the gravity along the Mid-Atlantic Ridge between $15^{\circ} \mathrm{N}$ and $40^{\circ} \mathrm{N}$ : Constraints for the thermal structure, submitted to Mid-Atlantic Ridge AGU Monograph, Maurice Ewing volume series, 1997.

Tilling, R. I., and J. J. Dvorak, Anatomy of a basaltic volcano, Nature, 363, 125-133, 1993.

Tivey, M. A., Fine-scale magnetic anomaly field over the southern Juan de Fuca Ridge: Axial magnetization low and implications for crustal structure, J. Geophys. Res., 99, 4833-4855, 1994.

Tivey, M. A., and H. P. Johnson, The central anomaly magnetic high: Implications for ocean crust construction and evolution, J. Geophys. Res., 92, 12,685-12,694, 1987.

Tivey, M. A., and H. P. Johnson, Variations in oceanic crustal structure and implications for the fine-scale magnetic anomaly signal, Geophys. Res. Lett., 20, 1879-1882, 1993.

Tolstoy, M., A. J. Harding, and J. A. Orcutt, Crustal thickness on the Mid-Atlantic Ridge: Bull's eye gravity anomalies and focused accretion, Science, 262, 726-729, 1993.

Tolstoy, M., A. J. Harding, J. A. Orcutt, and the TERA Group, Deepening of the axial magma chamber on the southern East Pacific Rise towards the Garrett Fracture Zone, J. Geophys. Res., 102, 3097-3108, 1997. 
Toomey, D. R., S. C. Solomon, and G. M. Purdy, Microearthquakes beneath median valley of Mid-Atlantic Ridge near $23^{\circ} \mathrm{N}$ : Tomography and tectonics, J. Geophys. Res., 93, 90939112, 1988.

Toomey, D. R., G. M. Purdy, S. C. Solomon, and W. S. D. Wilcock, The three-dimensional seismic velocity structure of the East Pacific Rise near latitude $9^{\circ} 30^{\prime} \mathrm{N}$, Nature, 347 , 639-645, 1990.

Toomey, D. R., S. C. Solomon, and G. M. Purdy, Tomographic imaging of the shallow crustal structure of the East Pacific Rise at $9^{\circ} 30^{\prime} \mathrm{N}, J$. Geophys. Res., 99, 24,135-24,157, 1994.

Triantafyllou, M. S., and F. S. Hover, Cable dynamics for tethered underwater vehicles, MIT SeaGrant College Program 90-4, Massachusetts Institute of Technology, 1990.

Tucholke, B. E., and J. Lin, A geological model for the structure of ridge segments in slow spreading ocean crust, J. Geophys. Res., 99, 11,937-11,958, 1994.

Tucholke, B. E., and H. Schouten, Kane fracture zone, Mar. Geophys. Res., 10, 1-39, 1988.

Tucholke, B. E., J. Lin, and M. C. Kleinrock, Megamullions and mullion structure defining oceanic metamorphic core complexes on the Mid-Atlantic Ridge, J. Geophys. Res., 103, 9857-9866, 1998.

Vera, E. E., and J. B. Diebold, Seismic imaging of oceanic layer $2 \mathrm{~A}$ between $9^{\circ} 30^{\prime} \mathrm{N}$ and $10^{\circ} \mathrm{N}$ on the East Pacific Rise from two-ship wide-aperture profiles, J. Geophys. Res., 99, 3031-3041, 1994.

Vera, E. E., J. C. Mutter, P. Buhl, J. A. Orcutt, A. J. Harding, M. E. Kappus, R. S. Detrick, and T. Brocher, The structure of 0 - to $0.2-\mathrm{m} . \mathrm{y}$-old oceanic crust at $9^{\circ} \mathrm{N}$ on the East Pacific Rise from expanded spread profiles, J. Geophys. Res., 95, 15,529-15,556, 1990.

Vine, F. J., and D. H. Matthews, Magnetic anomalies over oceanic ridges, Nature, 199, 947-949, 1963.

Vogt, P. R., Amplitudes of oceanic magnetic anomalies and the chemistry of oceanic crust: Synthesis and review of 'magnetic telechemistry', Can. J. Earth Sci., 16, 2236-2262, 1979.

Vogt, P. R., Magnetic anomalies and crustal magnetization, in The Western North Atlantic Region, edited by P. R. Vogt and B. E. Tucholke, vol. M of The Geology of North America, chapter 15, pp. 229-256, Geological Society of America, Boulder, CO, 1986.

Vogt, P. R., and H. P. Johnson, Magnetic telechemistry of oceanic crust?, Nature, 245, 373-375, 1973.

Watkins, N., Magnetic telechemistry is elegant but nature is complex, Nature, 251, 497-498, 1974. 
Weiland, C. M., K. C. Macdonald, and N. R. Grindlay, Ridge segmentation and the magnetic structure of the Southern Mid-Atlantic Ridge: $26^{\circ} \mathrm{S}$ and $31^{\circ}-35^{\circ} \mathrm{S}$ : Implications for magmatic processes at slow spreading centers, J. Geophys. Res., 101, 8055-8073, 1995.

White, D. J., and R. M. Clowes, Shallow crustal structure beneath the Juan de Fuca Ridge from 2-D seismic refraction tomography, Geophys. J. Int., 100, 349-367, 1990.

White, R. S., and G. M. Purdy, Crustal velocity structure on the flanks of the Mid-Atlantic Ridge at $24^{\circ} \mathrm{N}$, Geophys. J. R. Astron. Soc., 75, 361-385, 1983.

White, R. S., and R. B. Whitmarsh, An investigation of seismic anisotropy due to cracks in the upper oceanic crust at $45^{\circ} \mathrm{N}$, Mid-Atlantic Ridge, Geophys. J. R. Astron. Soc., 79, 439-467, 1984.

White, R. S., D. McKenzie, and R. K. O'Nions, Oceanic crustal thickness from seismic measurements and rare earth element inversions, J. Geophys. Res., 97, 19,683-19,715, 1992.

Whitehead, J. A., H. J. B. Dick, and H. Schouten, A mechanism for magmatic accretion under spreading centres, Nature, 312, 146-148, 1984.

Whitmarsh, R. B., Axial intrusion zone beneath the median valley of the Mid-Atlantic Ridge at $37^{\circ} \mathrm{N}$ detected by explosion seismology, Geophys. J. R. Astron. Soc., 42, 189$215,1975$.

Whitmarsh, R. B., Seismic refraction studies of the upper igneous crust in the North Atlantic and porosity estimates for layer 2, Earth Planet. Sci. Lett., 37, 451-464, 1978.

Whitmarsh, R. B., J. A. Orcutt, T. H. Jordan, R. G. Adair, and P. M. Shearer, Velocity bounds on the seismic structure of mesozoic crust and upper mantle in the southwest Pacific Basin from downhole observations at Deep Sea Drilling Hole 595B, Initial Rep. Deep Sea Drill. Proj., 91, 437-444, 1987.

Widess, M. B., How thin is a thin bed?, Geophysics, 38, 1176-1180, 1973.

Wilcock, W. S. D., S. C. Solomon, D. R. Toomey, and G. M. Purdy, The seismic attenuation structure of a fast-spreading mid-ocean ridge, Science, 258, 1470-1473, 1992.

Wilkens, R. H., G. J. Fryer, and J. Karsten, Evolution of porosity and seismic structure of upper oceanic crust: Importance of aspect ratios, J. Geophys. Res., 96, 17,981-17,995, 1991.

Wittpenn, N. A., C. G. A. Harrison, and D. W. Handschumacher, Crustal magnetization in the South Atlantic from inversion of magnetic anomalies, J. Geophys. Res., 94, 15,463$15,480,1989$.

Won, I. J., and M. Bevis, Computing the gravitational and magnetic anomalies due to a polygon: Algorithms and Fortran subroutines, Geophysics, 52, 232-238, 1987. 
Wooldridge, A. L., S. E. Haggerty, P. A. Rona, and C. G. A. Harrison, Magnetic properties and opaque mineralogy of rocks from selected seafloor hydrothermal sites at oceanic ridges, J. Geophys. Res., 95, 12,351-12,374, 1987.

Xenophontos, C., and J. G. Malpas, Excursion B, Part 2. Sheeted dikes and their relationships to pillow lavas and plagiogranite, in Field Excursion Guidebook, edited by C. Xenophontos and J. G. Malpas, Troodos 87-Symposium on Ophiolites and Oceanic Lithosphere, pp. 82-91, Geological Survey Department, Nicosia, Cyprus, 1987.

Zonenshain, L. P., M. I. Kuzmin, A. P. Lisitsin, Y. A. Bogdanov, and B. V. Baranov, Tectonics of the Mid-Atlantic rift valley between TAG and MARK areas (26-24ํ): Evidence for vertical tectonism, Tectonophysics, 159, 1-23, 1989. 